\title{
Age related differences and quality of life in rectal cancer surgery
}

Citation for published version (APA):

Orsini, R. G. (2016). Age related differences and quality of life in rectal cancer surgery: A step towards patient tailored rectal cancer treatment. [Doctoral Thesis, Maastricht University]. Maastricht University. https://doi.org/10.26481/dis.20161216ro

Document status and date:

Published: 01/01/2016

DOI:

10.26481/dis.20161216ro

Document Version:

Publisher's PDF, also known as Version of record

\section{Please check the document version of this publication:}

- A submitted manuscript is the version of the article upon submission and before peer-review. There can be important differences between the submitted version and the official published version of record.

People interested in the research are advised to contact the author for the final version of the publication, or visit the DOI to the publisher's website.

- The final author version and the galley proof are versions of the publication after peer review.

- The final published version features the final layout of the paper including the volume, issue and page numbers.

Link to publication

\footnotetext{
General rights rights.

- You may freely distribute the URL identifying the publication in the public portal. please follow below link for the End User Agreement:

www.umlib.nl/taverne-license

Take down policy

If you believe that this document breaches copyright please contact us at:

repository@maastrichtuniversity.nl

providing details and we will investigate your claim.
}

Copyright and moral rights for the publications made accessible in the public portal are retained by the authors and/or other copyright owners and it is a condition of accessing publications that users recognise and abide by the legal requirements associated with these

- Users may download and print one copy of any publication from the public portal for the purpose of private study or research.

- You may not further distribute the material or use it for any profit-making activity or commercial gain

If the publication is distributed under the terms of Article $25 \mathrm{fa}$ of the Dutch Copyright Act, indicated by the "Taverne" license above, 
Age related differences and quality of life in rectal

\section{cancer surgery}

A step towards patient tailored rectal cancer treatment 
No part of this thesis may be reproduced or transmitted in any form or by any means, without prior permission in writing by the author, or when appropriate, by the publishers of the publications.

Layout: Tiny Wouters

Cover: STUDIO BONKEND Branding + Design

Production: Ridderprint BV

ISBN: 978-94-6299-469-0

Financial support for publication of this thesis: Catharina Ziekenhuis Eindhoven, Maastricht University - GROW - School for Oncology \& Developmental Biology, Integraal Kankercentrum Nederland, Medtronic, Rabobank Eindhoven-Veldhoven, ChipSoft, EuroTec B.V., Olympus Nederland B.V. and Fysiotherapie de Smidse. 


\title{
Age related differences and quality of life in rectal
}

\author{
cancer surgery \\ A step towards patient tailored rectal cancer treatment
}

PROEFSCHRIFT

ter verkrijging van de graad van doctor aan de Universiteit Maastricht, op gezag van de rector magnificus Prof. dr. Rianne M. Letschert volgens het besluit van het College van Decanen, in het openbaar te verdedigen op vrijdag 16 december 2016 om 12:00 uur

door

Ricardo Giovanni Orsini

Geboren op 24 december 1986

te Geldrop 
Promotor

Prof. dr. H.J.T. Rutten

\section{Copromotores}

Dr. I.H.J.T. De Hingh (Catharina Ziekenhuis Eindhoven)

Dr. G.A.P Nieuwenhuijzen (Catharina Ziekenhuis Eindhoven)

\section{Manuscriptcommissie}

Prof. dr. L.P.S. Stassen (voorzitter)

Prof. dr. G.L. Beets

Dr. H.A.A.M. Maas (TweeSteden Ziekenhuis Tilburg)

Prof. dr. J.H.W. de Wilt (Radboudumc Nijmegen) 


\section{Contents}

Chapter 1 General introduction and outline of the thesis

Chapter 2 The modern anatomical surgical approach to localised rectal cancer

Part I Age related differences in outcome of rectal cancer

Chapter 3 Comparable survival for young rectal cancer patients, despite unfavourable morphology and more advanced stage disease

Chapter 4 Are there age related differences in morbidity and mortality after surgery for locally advanced and locally recurrent rectal cancer?

Part II Health-related quality of life and sexual function after treatment for rectal cancer

Chapter 5 Measuring the health-related quality of life and sexual functioning of patients with rectal cancer: does type of treatment matter?

Chapter 6 Does extended surgery influence health-related quality of life in patients with rectal cancer?

Chapter 7 Quality of life of older rectal cancer patients is not impaired by a permanent stoma

Part III The elderly rectal cancer patient

Chapter 8 Rectal cancer treatment in the elderly

Chapter 9 Surgery for rectal cancer what is on the horizon?

Chapter 10 Summary, discussion and future perspectives

Appendices Samenvatting

Dankwoord 

CHAPTER 1 General introduction and outline of the thesis

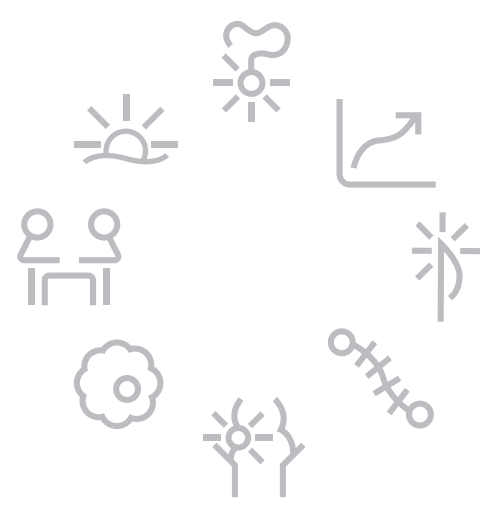


Chapter 1 
In the Netherlands colorectal cancer is the second most frequent type of cancer in male and third most common malignancy in women. ${ }^{1}$ Approximately one third of these patients have a rectal carcinoma. Rectal cancer mainly affects middle-aged and elderly individuals, with more than $75 \%$ of patients being diagnosed at 60 years or older. ${ }^{1,2}$ Although rectal cancer is mainly a disease of the older patient, there is an increasing incidence of patients diagnosed with rectal cancer at the age of $\leq 40$ years. ${ }^{3,4}$

In the early nineties, surgical technique changed to the complete removal of the rectum within its enveloping mesorectal fascia (TME surgery) and lead to major improvement in local recurrence rate. ${ }^{5}$ Further improvement in local control was observed after the Dutch TME trial, in which radiotherapy $(5 \times 5 \mathrm{~Gy})$ was administered prior to surgery. ${ }^{6}$ After implementation of both TME surgery and neoadjuvant radiotherapy in the Netherlands a significant improvement in local control was observed. ${ }^{7}$

In locally advanced rectal cancer (LARC), the tumour invades the mesorectal fascia or even penetrates into the surrounding pelvic structures. In this stage, TME surgery would inevitably lead to positive surgical margins. These patients require more extended resections, beyond the mesorectal fascia with removal of surrounding organs. Preoperative treatment with radiotherapy and concomitant chemotherapy can effectively downsize and even downstage locally advanced tumours and thus reduce the threat of an involved margin, allowing a more organ preservative approach. ${ }^{8-10}$ In LARC, long course pre-operative chemoradiation compared to short course preoperative radiotherapy significantly reduces local recurrence rates. ${ }^{11}$

Currently, the Dutch guidelines on treatment of rectal cancer are as follows ${ }^{12}$ :

- In case of cT1-3 without involvement of the mesorectal fascia ( $>1 \mathrm{~mm}$ distance), $\leq 5$ $\mathrm{mm}$ extramural invasion and no pathological lymph nodes treatment consists of TME surgery only.

- In case of cT1-3 with up to 3 positive lymph nodes or cT3NO without involvement of the mesorectal fascia ( $>1 \mathrm{~mm}$ distance) and with $>5 \mathrm{~mm}$ extramural invasion treatment consists of neo-adjuvant radiotherapy $(5 \times 5 \mathrm{~Gy})$ followed by TME surgery.

- In case of cT4 or cT3 with mesorectal fascia involvement ( $\leq 1 \mathrm{~mm}$ distance) or any cT stadium with $\geq 4$ pathological lymph nodes treatment consists of neo-adjuvant chemoradiation therapy followed by surgery after a waiting period of 8-12 weeks.

Rectal cancer treatment has evolved from a surgery-alone treatment to multimodality and multidisciplinary treatment. Using international guidelines and guidelines from consensus meetings, we currently stratify treatment strategies in order to obtain optimal oncological outcomes. The current multidisciplinary treatment for rectal cancer has led to decreased morbidity and a significant improvement of local control and survival. $^{13,14}$ 
Preoperative treatment with radiotherapy and concomitant chemotherapy can effectively downsize and even downstage locally advanced tumors and reduce the threat of an involved margin and might enable a more preservative approach. ${ }^{8,15}$ Despite neoadjuvant treatment, extended resections are not always avoidable. In the pelvis in which organs are packed tightly together, these extended resections often result in loss of autonomic nerves, other pelvic supporting structures (sacrum, pelvic floor muscles) or organs (bladder, genital organs and ureters). In addition, these resections are accompanied by relatively high morbidity rates (15-68\%). ${ }^{16-19}$

In rectal cancer and particularly in locally advanced or locally recurrent (LRRC) cases, treatment can have a tremendous effect on a patient's health-related quality of life (HRQL). For both the clinician as the patient it is extremely important to have knowledge on the influence of treatment strategies on patients' HRQL. In some cases, patients might have other priorities in life, making oncological outcome less important. It can be hypothesized that the best oncological treatment is not always worthwhile at all costs. In order to achieve a common goal and shared decision making, instead of a clinician oncological outcome driven decisions, HRQL after treatment needs to be actively discussed. Especially in the elderly patient, in whom expectations of quality of life are more relation to life expectancy, rather than oncological outcome, the integration of $\mathrm{HRQOL}$ issues plays an even more important role in the decision making process.

Even though the importance of patient-reported outcomes and quality of life are increasingly recognized, research on LARC or LRRC has predominantly focused on assessing local or distance control and overall survival. In addition, also in expert consensus meetings such as the EUropean REgistry of Cancer Care (EURECCA) or Beyond the TME Collaborative influence of treatment on patients quality of life was not actively discussed. ${ }^{20,21}$ The next step towards a more individualised approach will be to optimise treatment for the individual patient, who is interested not only in the oncological outcome but also in functional results and subsequent quality of life.

\section{Aim and outline of the thesis}

This thesis addresses several issues in rectal cancer treatment and can be divided into three parts. Preceding these parts an overview is provided on the modern anatomical approach to localized rectal cancer (Chapter 2). It addresses the importance of a multidisciplinary approach with good quality pathology, importance of pre-operative imaging and role of neo-adjuvant treatment. In addition, the surgical anatomy and importance of the surgical approach are described in more detail. 
Part I of this thesis describes age-related differences in outcome of rectal cancer. In Chapter 3 differences in tumour characteristics, treatment and survival in patients aged $\leq 40$ years and $41-70$ years were studied. For this purpose, data of the Netherlands Cancer Registry (NCR) were used. The NCR collects data on all newly diagnosed patients with cancer in the Netherlands since 1989. In Chapter 4 age related differences in morbidity and survival after treatment for LARC and LRRC were studied. All patients included in this study were operated upon in the Catharina Hospital which serves as a national referral center for locally advanced and locally recurrent rectal cancer. The primary aim of this study was to describe the morbidity and mortality of cT4 and locally recurrent rectal cancer in the elderly patient who is treated with curative intent. In order to investigate differences in occurrence and consequences of complications, morbidity and mortality were compared with younger patients. Secondary risk factors for one-year mortality were studied.

Part II of this thesis focusses on patients' HRQL and sexuality after treatment for rectal cancer. Several treatment related issues were studied with a special interest for age related differences. In Chapter $\mathbf{5}$ differences in HRQL between various disease stages were investigated. A comparison was made between patients with non-locally advanced disease (NAD), LARC and LRRC. Furthermore, a comparison with a healthy (normative) population was performed. Chapter 6 describes the influence of extended resections (outside mesorectal fascia) on HRQL in LARC. Patients were divided into TME or extended surgery and further stratified by age ( $<70$ and $\geq 70$ years). Chapter 7 studies the influence of a permanent stoma on HRQL. Knowledge of the impact of a stoma on the HRQL may help to determine a treatment strategy for elderly patients suffering from rectal cancer that is both safe and preserves a good HRQL. In rectal cancer, patients typically undergo a sphincter preserving procedure (low anterior resection: LAR) with often a temporary stoma or an abdominoperineal resection (APR) resulting in a permanent colostomy. The choice for one of these procedures depends on the level of the tumour, the technical feasibility to perform an anastomosis and the condition of the patient. For this study, data from the Eindhoven Cancer Registry (ECR) was used, which covers a population of 2.4 million inhabitants in the Southern part of the Netherlands. Patients with low rectal cancer $(\leq 10 \mathrm{~cm}$ from anal verge) operated upon in 4 regional hospitals were selected. Patients with a permanent stoma were compared to those without a stoma and were further stratified by age $<70$ and $\geq 70$ years). These groups were also compared to a normative population.

Part III of this thesis focusses on the elderly rectal cancer patient. Elderly rectal cancer patients represent a heterogeneous group ranging from the active and fit to very frail patients, who are at high risk of treatment complications. In addition, when compared to younger patient, elderly patients have other expectations of life with respect to their life expectancy and HRQL. For the clinician elderly patients can be a challenging group 
to treat because trials and current national and international guidelines do not specifically focus on elderly. In Chapter $\mathbf{8}$ a guide for treatment of elderly rectal cancer is proposed on the basis of the available literature on elderly rectal cancer patients.

In Chapter 9 future perspectives are discussed with a special focus on registries, auditing and shared decision making. In Chapter 10 a summary of the main results is given. In addition, clinical implications of this thesis for current practice and suggestions for future research are discussed. 


\section{References}

1. IKNL Cijfers over kanker, http://www.cijfersoverkanker.nl/selecties/dataset_1/ img56bb0a93ce659?language=en, accessed on 22-11-2015.

2. Atkin WS, Cuzick J. Screening for Colorectal Cancer. Lancet 1993; 341(8861): 1667.

3. Davis DM, Marcet JE, Frattini JC, Prather AD, Mateka JJ, Nfonsam VN. Is It Time to Lower the Recommended Screening Age for Colorectal Cancer? J Am Coll Surg 2011;213:352-61.

4. Meyer JE, Narang T, Schnoll-Sussman FH, Pochapin MB, Christos PJ, Sherr DL. Increasing Incidence of Rectal Cancer in Patients Aged Younger Than 40 Years: an Analysis of the Surveillance, Epidemiology, and End Results Database. Cancer 2010;116:4354-9.

5. Heald RJ, Ryall RD. Recurrence and Survival After Total Mesorectal Excision for Rectal Cancer. Lancet 1986;1:1479-82.

6. Peeters KC, Marijnen CA, Nagtegaal ID, et al. The TME Trial After a Median Follow-Up of 6 Years: Increased Local Control but No Survival Benefit in Irradiated Patients With Resectable Rectal Carcinoma. Ann Surg 2007;246:693-701.

7. den Dulk M, Krijnen P, Marijnen CA, et al. Improved Overall Survival for Patients With Rectal Cancer Since 1990: the Effects of TME Surgery and Pre-Operative Radiotherapy. Eur J Cancer 2008;44:1710-6.

8. Valentini V, van Stiphout RG, Lammering G, et al. Nomograms for Predicting Local Recurrence, Distant Metastases, and Overall Survival for Patients With Locally Advanced Rectal Cancer on the Basis of European Randomized Clinical Trials. J Clin Oncol 2011;29:3163-72.

9. Sauer R, Liersch T, Merkel S, et al. Preoperative Versus Postoperative Chemoradiotherapy for Locally Advanced Rectal Cancer: Results of the German CAO/ARO/AIO-94 Randomized Phase III Trial After a Median Follow-Up of 11 Years. J Clin Oncol 2012;30:1926-33.

10. Bosset JF, Calais G, Mineur L, et al. Enhanced Tumorocidal Effect of Chemotherapy With Preoperative Radiotherapy for Rectal Cancer: Preliminary Results--EORTC 22921. J Clin Oncol 2005;23:5620-7.

11. Ceelen WP, Van NY, Fierens K. Preoperative Chemoradiation Versus Radiation Alone for Stage II and III Resectable Rectal Cancer. Cochrane Database Syst Rev 2009;(1): CD006041.

12. Dutch National Guidelines Rectal Cancer. http://www.oncoline.nl/colorectaalcarcinoom. accessed on 22-11-2015.

13. Kusters M, Valentini V, Calvo FA, et al. Results of European Pooled Analysis of IORT-Containing Multimodality Treatment for Locally Advanced Rectal Cancer: Adjuvant Chemotherapy Prevents Local Recurrence Rather Than Distant Metastases. Ann Oncol 2010;21:1279-84.

14. den Dulk M, Krijnen P, Marijnen CA, et al. Improved Overall Survival for Patients With Rectal Cancer Since 1990: the Effects of TME Surgery and Pre-Operative Radiotherapy. Eur J Cancer 2008;44:1710-6.

15. Bujko K, Kepka L, Michalski W, Nowacki MP. Does Rectal Cancer Shrinkage Induced by Preoperative Radio(Chemo)Therapy Increase the Likelihood of Anterior Resection? A Systematic Review of Randomised Trials. Radiother Oncol 2006;80:4-12.

16. Rahbari NN, Ulrich AB, Bruckner T, et al. Surgery for Locally Recurrent Rectal Cancer in the Era of Total Mesorectal Excision: Is There Still a Chance for Cure? Ann Surg 2011;253:522-33.

17. Dresen RC, Gosens MJ, Martijn H, et al. Radical Resection After IORT-Containing Multimodality Treatment Is the Most Important Determinant for Outcome in Patients Treated for Locally Recurrent Rectal Cancer. Ann Surg Oncol 2008;15:1937-47.

18. Vermaas M, Ferenschild FT, Verhoef C, et al. Total Pelvic Exenteration for Primary Locally Advanced and Locally Recurrent Rectal Cancer. Eur J Surg Oncol 2007;33:452-8.

19. Peeters KC, Tollenaar RA, Marijnen CA, et al. Risk Factors for Anastomotic Failure After Total Mesorectal Excision of Rectal Cancer. Br J Surg 2005;92:211-6.

20. van de Velde CJ, Boelens PG, Borras JM, et al. EURECCA Colorectal: Multidisciplinary Management: European Consensus Conference Colon \& Rectum. Eur J Cancer 2014;50:1.

21. Consensus Statement on the Multidisciplinary Management of Patients With Recurrent and Primary Rectal Cancer Beyond Total Mesorectal Excision Planes. Br J Surg 2013;100:E1-33. 


\section{CHAPTER 2}

The modern anatomical surgical approach to localised rectal cancer

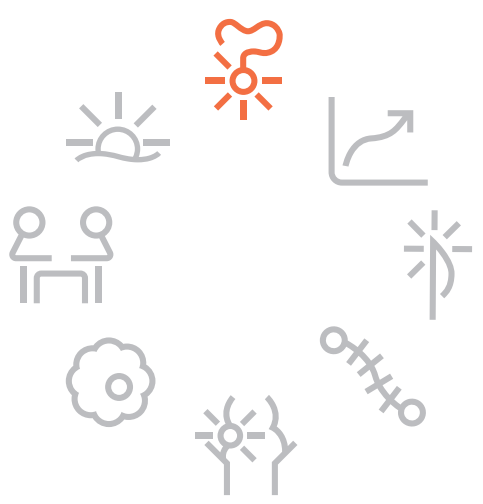

R.G. Orsini

T. Wiggers

M.C. DeRuiter P. Quirke

R.G. Beets-Tan

C.J. van de Velde

H.J. Rutten 
On a worldwide scale, colorectal cancer is one of the leading causes of cancer deaths affecting millions of people every year. One third of colorectal cancer concerns the rectum. In more than two third of the cases rectal cancer is still localized to the pelvis without detectable metastases. In these cases surgical resection is the cornerstone for a curative approach. Since the introduction of the combined abdomino-perineal resection by Miles and Quénu around the beginning of the twentieth century, ${ }^{1}$ rectal cancer became a curable disease. However, for many decades results of surgery have been disappointing as it was often spoiled by a local recurrence rate of up to $40 \%$ or even higher. Uncontrolled progressive local recurrences, hardly palliated by irradiation or chemotherapy, have brought a miserable death to tens of millions of patients.

This situation lasted till the end of the last century when the anatomical basis of rectal cancer surgery was revived by Heald and Quirke. ${ }^{2,3}$ Quirke demonstrated that the radial margin between tumour border and surgical resection margin was a strong prognosticator for local recurrence. He pointed out that both tumour progression and surgical quality were important for a safe margin. Poor surgery with incomplete mesorectum or tears into the mesorectal fat or muscular tube of the rectum could reduce this margin and consequently lead to local recurrences. Heald introduced the principle of total mesorectal excision (TME). By doing so he defined the optimal quality of surgery.

Worldwide surgeons have accepted as standard of care that optimally the rectum has to be removed within its enveloping mesorectal fascia. TME emphasizes the importance of an anatomical resection in the planes between the mesorectal fascia and the surrounding pelvic fascias. However, the principle of the resection of the rectum within its mesorectal fascia seems to fail when analysing low rectal cancer. From the early randomised controlled trials it was learned that patients requiring an abdominoperineal excision (APE) still had high positive circumferential resection margins. ${ }^{4-7}$ The lower rectum and anorectum are not surrounded by a protecting layer of mesorectal fat. Instead, already in an early stage, progressing tumours reach and possibly infiltrate the pelvic floor muscles, which are continuous with the external sphincter more distally. Compared with patients undergoing low anterior resection (LAR) APE patients have tumours located lower and more advanced, therefore new principles of surgery had to be developed. ${ }^{8}$ Results of lower rectal cancer surgery improved when the principle of the extra levator approach was introduced. ${ }^{9-12}$ This involves removal of the lower rectum during an abdomino-perineal excision en bloc with the external sphincter and levator ani muscles. In the lower rectum the role of complete removal of the mesorectal fascia is replaced by removal of the levator ani muscles. Again, the quality of surgery can be judged by the completeness of this resection.

Modern rectal cancer surgery can be tailored to the specific topographical relations of the tumour. In proximal tumours the mesorectal fascia acts as the guiding structure. 
Transection of the specimen can be performed $4-5 \mathrm{~cm}$ distally from the lower tumour border or at the pelvic floor when the mesorectum terminates higher. More distal tumours can be removed either intersphincteric; if the tumour is confined within the smooth muscle tube of the muscularis propria, sometimes even allowing for a colo-anal anastomosis; or extralevator if the pelvic floor is threatened or already in involved by tumour progression. The third option for an APE is to do an even wider approach taking out the ischiorectal fat as well en bloc with the levator muscles, if the tumour has perforated or fistulated through the pelvic floor muscles into this fatty area. However, this will be only the case in extremely rare circumstances.

Modern rectal cancer surgery is part of a multidisciplinary approach. Preoperative imaging with magnetic resonance imaging (MRI) is able to delineate the tumour very accurately and helps to select those patients requiring downsizing and downstaging, optimising the chances for a good tumour resection. ${ }^{13-15}$ The pathologist plays an important role in the feedback to the surgeon, which is necessary to improve surgical outcome. $^{16}$

The first step in integration of optimal imaging, treatment modalities and pathology is taken is several countries. The next step will be to optimise treatment for the individual patient, who is not only interested in the oncological outcome, but also in functional results and subsequent quality of life. Avoiding and decreasing morbidity, especially in the elderly, will require the development of new innovative strategies.

\section{Contribution of pathology to the anatomical approach}

It may seem odd to start a discussion on modern surgical approach to localised rectal cancer with the findings of the pathologist. However, it was a pathologist, who demonstrated the importance of the distance of the radial tumour border to the mesorectal fascia, which is called the circumferential resection margin (CRM) in TMEsurgery. ${ }^{3}$

In 2002 Nagtegaal analysed the data of the Dutch TME-study, and she confirmed that in $44 \%$ of the patients the involved circumferential resection margin was the result of poor quality surgery. It was also shown that, after incomplete mesorectal excision the overall recurrence rate was almost doubled, which could mainly be attributed to the excess of local recurrences. ${ }^{16}$ Nagtegaal and Quirke performed a meta analysis on the importance of the CRM in more than 17.500 patients and concluded that CRM involvement predicts not only local recurrence, but also distant metastasis, and subsequent overall survival. Failure to achieve a negative CRM after neoadjuvant treatment leads to a poorer prognosis compared to patients without neoadjuvant 
treatment. Possibly the explanation for this is the selection of patient with tumours more resistant to therapy. This finding could be an argument for restaging after neoadjuvant therapy, and to consider more prolonged neoadjuvant treatment or to refer to a specialised centre for more extended resection or additional boosting of the area at risk. ${ }^{17}$

Thus, the actual feedback of the pathologist to the surgeon should contain information on the CRM and quality of surgery. ${ }^{18}$ An important other issue, which will be discussed later in this paper, is the evaluation of effectiveness of the chosen neoadjuvant treatment. Preferably, macroscopic images of the resected specimens as well as the microscopic images, should be available for internal audit and continued education and improvement of all member decisions during the multidisciplinary tumour board meetings.

Quirke proposed a 3-point grading system for the evaluation of the macroscopic specimen for both low anterior and abdomino-perineal resections. Good surgery would be qualified by an intact mesorectal fascia with only minor irregularities, or in case of APE, a specimen with levator ani and external sphincters without any defects deeper than $5 \mathrm{~mm}$ and the levator ani attached to the mesorectal fascia. ${ }^{19,20}$ See figure 2.1-2.3. After moderate quality of surgery the bulk of the mesorectum is removed but shows an irregular surface however still without exposing the muscularis propria or perforations. In case of an APE, a specimen which shows waist formation, indicative for a less complete levator ani covering at the anorectal junction, but with intact sphincters signifies a moderate quality of surgery.

Poor surgery would be characterized by severe irregularities on the surface of the specimen, exposing the muscularis propria or internal sphincter or even show perforations to the lumen. Very essential for the grading of the APE-specimen is the question if the levator ani muscle is still attached to the mesorectum. Thus, waist formation is avoided and the result is a more cylindrical resection. In order to achieve optimal feedback, pathology reports should be standardised, not only regarding the reporting of the TNM-status, but also on the quality of surgery. ${ }^{19}$ 


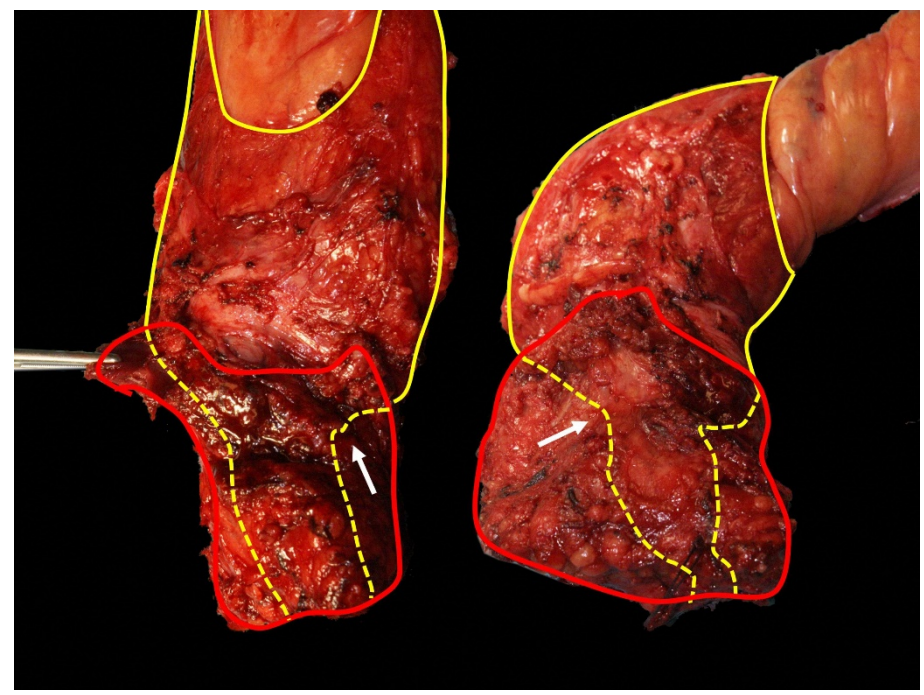

Figure 2.1 Rectal extralevator abdomino-perineal excision specimen. The solid yellow lines indicate the intact mesorectum. The red lines demonstrate the extralevator muscles attached to the specimen covering widely the anorectal junction (white arrow), where the mesorectum ends and the internal sphincter starts (dotted yellow line). Even advanced T3 or T4 tumours at the anorectal junction and below can be safely removed when covered by the extra levator muscle layer. Typically a cylindrical shape specimen. Mesorectum intact

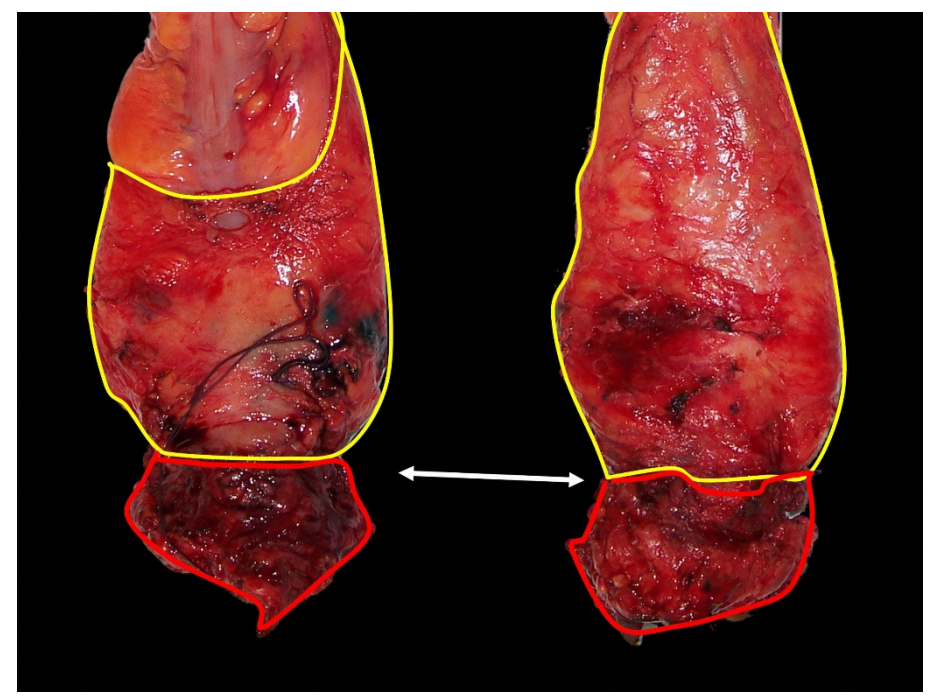

Figure 2.2 "Standard" abdomino-perineal excision specimen. Demonstrating waist formation at the anorectal junction or just above (white arrow). Only marginal coverage by the external sphincters of the distal mesorectum. Will suffice for less advanced tumours of the anorectum or anorectal junction. Specimen characterized by "waist" formation. Mesorectum intact 


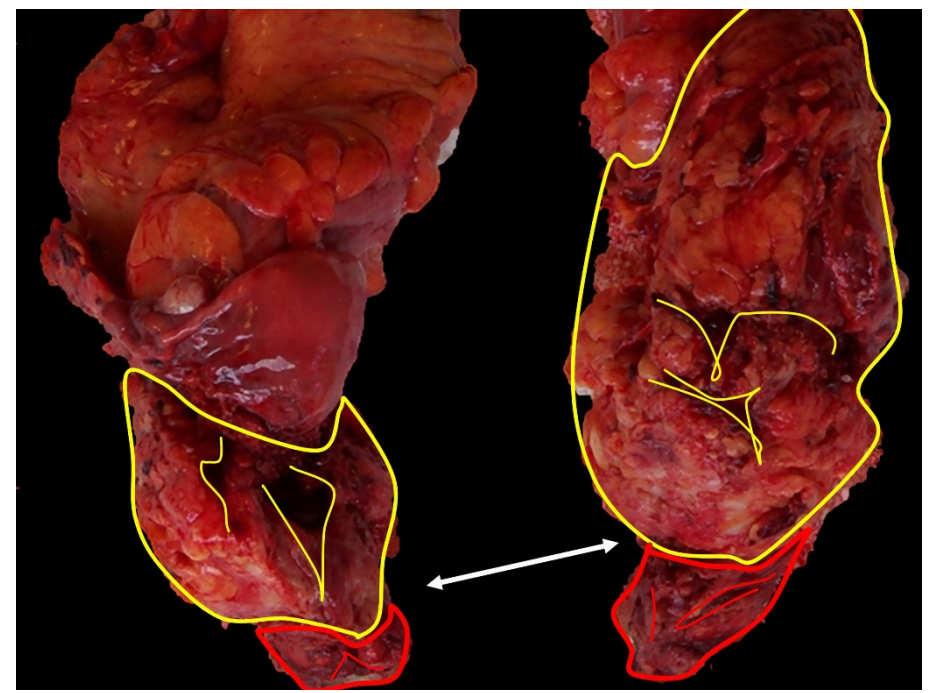

Figure 2.3 Poor specimen after abdomino-perineal excision. Deep indentations and even perforation in mesorectum. Very little coverage of the external sphincter and showing tears in the external sphincter.

\section{The importance of MRI for the surgical treatment of rectal cancer}

MRI is a reliable diagnostic tool for clinical staging of rectal cancer, but other imaging methods for the pelvis are also being used for this purpose. Computed tomography (CT) is able to identify enlarged lymphnodes although it is not accurate to assess the morphology of these nodes. Furthermore the contrast resolution of CT is insufficient to reliably assess involvement of the surgical resection plane in mid and lower rectal cancer. CT, however is indicated for distant staging of metastatic disease and, if there is no easy access to an MRI, for assessment of resectability of high rectal tumours. ${ }^{21-23}$ Endorectal ultrasound (EUS) cannot visualize the mesorectal fascia, but is the modality of choice to differentiate between T1 and T2 lesions for the selection of local therapies. EUS has a high sensitivity to stage depth of submucosal involvement. ${ }^{24}$ However, MRI is the king of kings of all imaging modalities in its tissue contrast resolution and provides the necessary detailed anatomical information on pelvic fascias and dissection planes between pelvic soft tissues, which sets the scene for the planning of the resection.

Without the anatomical topographical information of an MRI, the surgeon has to rely on ad hoc decisions when unexpected problems occur during the actual surgical procedure, and in a worst case scenario these problems may even go unnoticed, or have become irreversible. With the anatomical information from MRI critical sites of 
resection can be anticipated and addressed before surgery: i.e. use of neoadjuvant treatment or referral to a centre specialized in extended extra anatomical pelvic resection if TME surgery is not justified.

The Mercury study group reported the reliability of MRI on predicting extramural depth of tumour invasion. Very good correlation of extra mural spread on MRI and histopathology was found: the $95 \%$ confidence interval being less than $0.5 \mathrm{~mm}$. The TNM classification lacks specificity in the T3 stage. ${ }^{25}$ A T3 tumour with limited extramural (outside the muscularis propria) spread has a different prognosis compared to a T3 tumour with more extended spread. Merkel et al. demonstrated that a cut off point of $5 \mathrm{~mm}$ spread divided patients into groups with good and poor prognosis, provided that a safe CRM was obtained. ${ }^{26}$ For the surgeon it is important that patients with limited extramural spread can be treated as T2 patients, and in most cases will not require neoadjuvant treatment with its associated adverse effect on surgical morbidity and functional outcome.

Even more important from the surgical technical point of view is the fact that MRI can reliably anticipate an involved circumferential resection margin. MRI differentiates between high-risk ( $<1 \mathrm{~mm}$ ) and low-risk $(>1 \mathrm{~mm})$ patients for local recurrence. MR CRM margins $>1 \mathrm{~mm}$ and $<2 \mathrm{~mm},>2$ and $<5$ and $>5 \mathrm{~mm}$ carried a similar risk for local recurrence of around $7 \%$ in contrast to the $20 \%$ risk of patients with an anticipated margin of $<1 \mathrm{~mm} .{ }^{27}$ The ability to predict a 1 millimetre free margin was recently confirmed by a German group. ${ }^{28}$

The ability of MRI to discriminate between positive and negative lymphnodes is quite disappointing. Like other imaging modalities, conventional MRI without any MR contrast, lacks both sensitivity and specificity to identify or rule out positive nodes and cannot reliably be used for treatment stratification. ${ }^{29,30}$ Size has been an unreliable variable to predict nodal involvement. ${ }^{31,32}$ Several contrast agents have been and are under investigation, but at the time of writing results are still inconclusive. ${ }^{33-35}$ Diffusion-weighted MRI imaging (DWI) shows a high signal in both benign and malignant nodes and therefore cannot differentiate between the two. A restaging MRI, including DWI has a high negative predictive value (NPV) for the detection of nodal metastases and can be more reliably used for nodal restaging after neoadjuvant treatment. $^{36}$ The most reliable variable seems to be the evaluation of nodal morphology, like roundness, irregular border or heterogeneous texture. However, this is difficult and subject to large inter-observer variability, especially in nodes $<6 \mathrm{~mm}$ and in patients with only small sized nodes of limited value in clinical practices. ${ }^{31,37} \mathrm{MRI}$ plays an important role in the evaluation of response to neoadjuvant treatment (NT) and the consequences for the final surgical resection. NT is able to downsize the tumour. In particular, large tumours may have been over staged at the initial MRI. 
Sometimes a pushing tumour border see on primary staging MRI may be mistaken for an infiltrating one. Restaging with MRI after NT may reveal surgical dissection planes, which were obscured at the primary staging MRI. As a consequence, surgical planning can be more conservative.

Clinical T and N stage may also alter after NT and allow a change in surgical approach. MRI is furthermore accurately correlated with histopathologic downgrading of the tumour. Apart from opening new possibilities for a minimal invasive surgical approach for the very good responders, non-responders can be identified who may require intensification of the treatment plan. ${ }^{38-45}$ In order to understand the consequences of NT, it is of crucial importance that radiologists and pathologists participate in the multidisciplinary treatment team.

\section{Role of neoadjuvant treatment for the surgeon}

The primary objective in rectal cancer surgery is to achieve a free surgical resection margin. The purpose of NT is twofold: first, to sterilize the potential tumour cell bearing volume in the pelvis, which is not removed during surgery, more specifically the lateral zones of lymphatic spread; and second to change the size and stage of the primary rectal cancer in order to facilitate surgical resection and even to allow for more limited surgery. In Japan the lateral lymphnodes are removed as standard procedure during rectal cancer surgery. By doing so, NT can be safely omitted according to the Japanese. Comparison between the Japanese results with extended lymphadenectomy and the Dutch TME study, which randomized between TME surgery with and without $5 \times 5$ Gy preoperative radiotherapy showed that $5 \times 5$ with standard TME surgery was as effective as extended lateral lymphadenectomy for the prevention of local recurrences. $^{46}$

The Swedish Rectal Cancer Trials, the Dutch TME study and the British CRO7 study have clearly demonstrated that preoperative $5 \times 5$ Gy followed by immediate surgery yields excellent oncologic results in patients in whom a CRM negative margin can be achieved. In these studies, $5 \times 5$ was followed by immediate surgery (preferably within 1 week). ${ }^{47-50}$ A recent update of the third Swedish Rectal Cancer Trial shows that a waiting period after $5 \times 5$ Gy short course radiotherapy effectively reduces postoperative morbidity, while also a downstaging effect was noticed. ${ }^{51}$

In contrast, advanced tumours, invading the mesorectal fascia, or even penetrating into the surrounding pelvic structures would inevitably lead to positive surgical margins if the surgeon sticks to the principle of dissection along the mesorectal fascia. These patients require a more extended resection, peripheral to the mesorectal fascia. In the 
pelvis with organs packed tightly together these extended resections often result in loss of autonomic nerves, other pelvic supporting structures (sacrum, pelvic floor muscles) or organs (bladder, genital organs, ureters). Preoperative treatment with radiotherapy and concomitant chemotherapy can effectively downsize and even downstage locally advanced tumours and thus take away the threat of an involved margin, allowing for a more preservative approach. ${ }^{52-54}$ Whereas the lateral margin is influenced by NT, it is not evident that the distal margin moves upward, or that it is possible or even wise to perform a low anastomosis in a previously irradiated part of the (ano-)rectum. ${ }^{55,56}$ Systemic chemotherapy may also be incorporated into a NT scheme. In metastasized patients it helps to select the responders, who may be good candidates for metastasectomy as well as resection of the primary from those patients who are progressive and would not benefit from extended surgery. ${ }^{57}$ The Dutch Colorectal Cancer Group initiated the international RAPIDO study, which seeks to find answers for the question of whether upfront systemic chemotherapy as part of NT can reduce the occurrence of metastases in localised rectal cancer. ${ }^{58}$

\section{The anatomical surgical approach to localised rectal cancer}

The surgical approach is based on the preoperative MRI image and may also take into account the response to neoadjuvant treatment. The resection itself follows anatomical principles and is based on removal of the rectum within its covering mesorectal fascia. In proximal tumours, the distal rectum may be preserved, provided at least $4-5 \mathrm{~cm}$ of mesorectum is removed distally from the tumour. ${ }^{59}$ In low rectal cancers at the anorectal junction or below, depending on the infiltration depth of the tumour, the pelvic floor muscles and external sphincter often need to be removed en bloc with the rectum to assure a complete resection with a CRM of $>1 \mathrm{~mm} \cdot{ }^{10,11}$

A secondary objective is to avoid damage to the nerve system as little as possible. The pelvic autonomic nerves consist of a fine network originating around the aorta, which descends as a fine mesh lining the mesorectal fascia. The hypogastric nerves condense and split into two lateral bundles which can be easily identified and followed to the inferior hypogastric plexus. In this area innervation, lymphatic drainage and blood supply mingle in the lateral pillars of the rectum. The nervi erigentes also join the inferior hypogastric plexus from dorsolateral and also lie in close approximation to the dorsolateral mesorectal fascia. The somatic levator ani and pudendal nerves are protected by the pelvic fascia and less at risk than the autonomic nerves. ${ }^{60}$

The anterior mesorectum, distally to the peritoneal reflection, is thin, but, similarly to the rest of the mesorectum, also covered with a fascia like structure (fascia of Denonvilliers), which allows for dissecting the anterior mesorectal fascia from the 
prostate/vesicles or posterior vaginal wall. ${ }^{61,62}$ More distally, this layer ends and is replaced by intertwining bundles of somatic perineal muscles joining with the smooth muscular layer of the muscularis propria of the anorectum. This organisation of muscle fibres anchors the anorectum to the pelvis. More laterally and dorsally at the level of the sphincters the adherence of the smooth muscles to the surrounding external sphincter muscles is much more loosely organised. The external sphincter may be considered the distal part of the funnel shaped pelvic floor muscles enveloping the smooth muscular layer of the internal sphincter. As mentioned above, laterally and dorsally the adherence between internal and external sphincter is low and allows for the development of an intersphincteric resection plane. On the anterior side the somatic pelvic floor muscles which distally join in a tendinous perineal body, are very adherent to the anterior part of the anal rectum. Therefore no such thing as an intersphincteric dissection plane is present on the anterior side of the distal rectum. ${ }^{63}$ This is a very important anatomical fact which influences the way low rectal tumours can be dissected. An APE can be performed in three dissection planes: (1) the intersphincteric plane which is close to the internal sphincter and only suitable for tumours which are confined to the muscularis propria of the rectum; (2) the extra levator plane which follows the external fascia of the external sphincter continuous along the external fascia of the levator ani muscles and transects these muscles as lateral as possible before entering the abdomen; (3) the ischiorectal plane which also removes the ischiorectal fat and which follows the external fascia of the pelvis removing the ischiorectal fat en bloc with the levator ani muscles. Again, the abdomen is entered as laterally as possible at the level of the attachment of the levator ani muscles to the pelvic wall. (See Figure 2.4)

In most distal rectal carcinomas the extralevator abdomino perineal excision (ELAPE) is recommended to achieve a complete resection with a negative CRM. ${ }^{64}$ The patient may be either operated in supine position with the legs in movable stirrups or the patient may be turned to prone position for the perineal resection. ${ }^{65}$ When the operation is performed in supine position the patient does not need to be turned and the procedure can either start with the perineal phase or the abdominal phase.

In supine position the dissection starts with an incision around and subsequent closure of the anus. (Figure 2.5) 10,19 $^{10}$ The external perineal fascia which covers the external sphincter can be followed until the lateral attachment of the levator ani to the pelvic sidewall. At this level the levator ani can be cut exposing the mesorectum. Dorsally, the anococcygeal ligament has to be transected. Depending on the location of the tumour, the presacral space may be entered ventrally to the coccyx, or in dorsally located tumours, the coccyx may be removed to enter the presacral space. After transection of the levator on both sides, exposing the mesorectum and opening the presacral space exposing the dorsal part of the distal mesorectum, the anterior dissection may 
commence. The anterior part of the levator ani muscle encloses the internal genital organs and needs to be transected at the level of Denonvilliers' fascia. After exposing Denonvilliers' fascia the dissection continues distally. retracting the specimen dorsally helps to identify the somatic perineal muscles, which are closely adherent to the anorectum. The transection takes place in the somatic muscles avoiding a fausse route into the bowel. If the operation was not started with the abdominal phase, the abdomen is opened now. Dissection is according to TME principles avoiding nerve damage. As the pelvic floor muscles are already transected, taking out the specimen is a relative uncomplicated procedure.

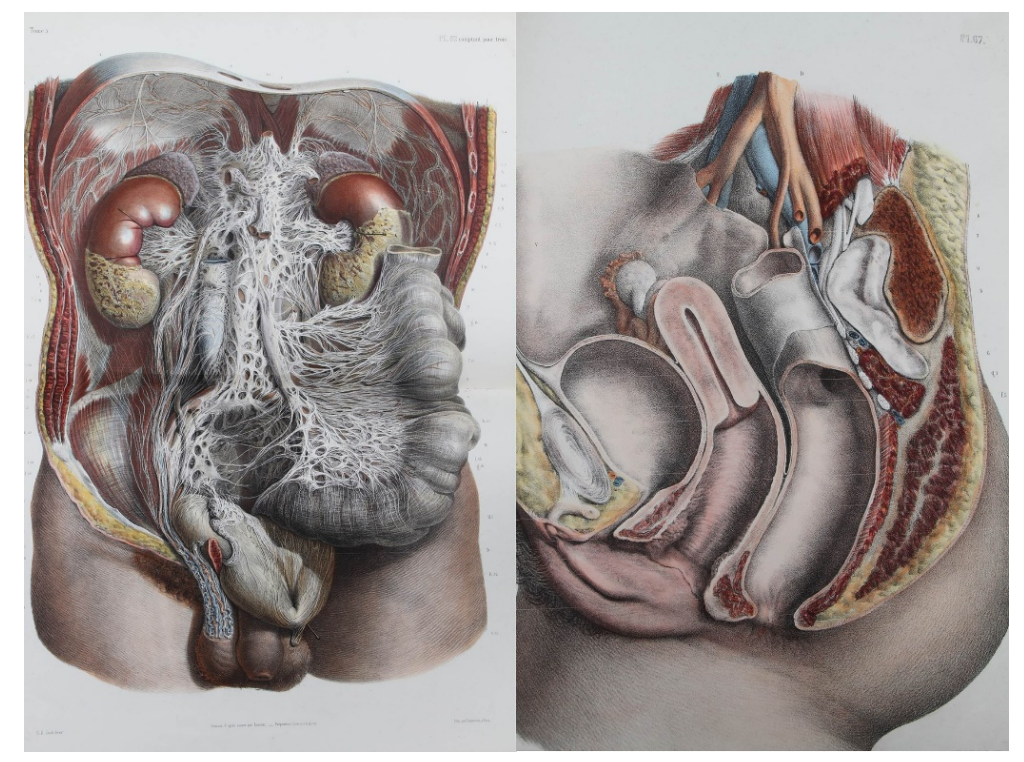

Figure 2.4 Early $19^{\text {th }}$ century anatomical lithography's. Demonstrating the intense autonomous nerve network in the abdomen, illustrating the high risk of surgery to damage the nerves. Furthermore the levator ani muscle can be seen as an envelope around the distal rectum.

If the operation is performed in prone position the procedure most often start with the abdominal phase with the patient lying in supine position. ${ }^{66}$ (Figure 2.6). Again, care must be taken not to push the dissection too deep down because of the risk of coning in, resulting in dissection of the pelvic floor off the mesorectum and subsequent waist formation. However, it is important to develop the presacral space until the os coccygis is exposed. On the lateral side the low hypogastric plexus has to be dissected off the mesorectum and the lateral pillars also have to be transected. Denonvilliers' fascia has to be exposed before the abdominal phase can be ended and the patient can be turned into prone position for the perineal phase. 
In prone position a teardrop like incision is made around the anal skin and extended proximally above the ano-coccygeal joint. After closure of the anus, the deep perineal fascia is followed from the external sphincter until the lateral attachments of the levator ani. After the coccyx has been cut, the already opened presacral space is entered and the lateral attachments of the levator ani can be cut. After arriving at the level of Denonvilliers' fascia, the specimen can be everted through the perineal wound and the dissection of the anterior plane of the specimen commences under direct vision. First, the puborectal sling has to be cut, as also the deep perineal muscles which are closely adherent to the anterior part of the anorectum. Again the dissection is carried out from proximally to distally. Cutting the perineal body is the last part of the operation before taking out the specimen. Care must be taken not to damage the urethra and the neural bundles of Walsh, which are very close to this dissection plane.

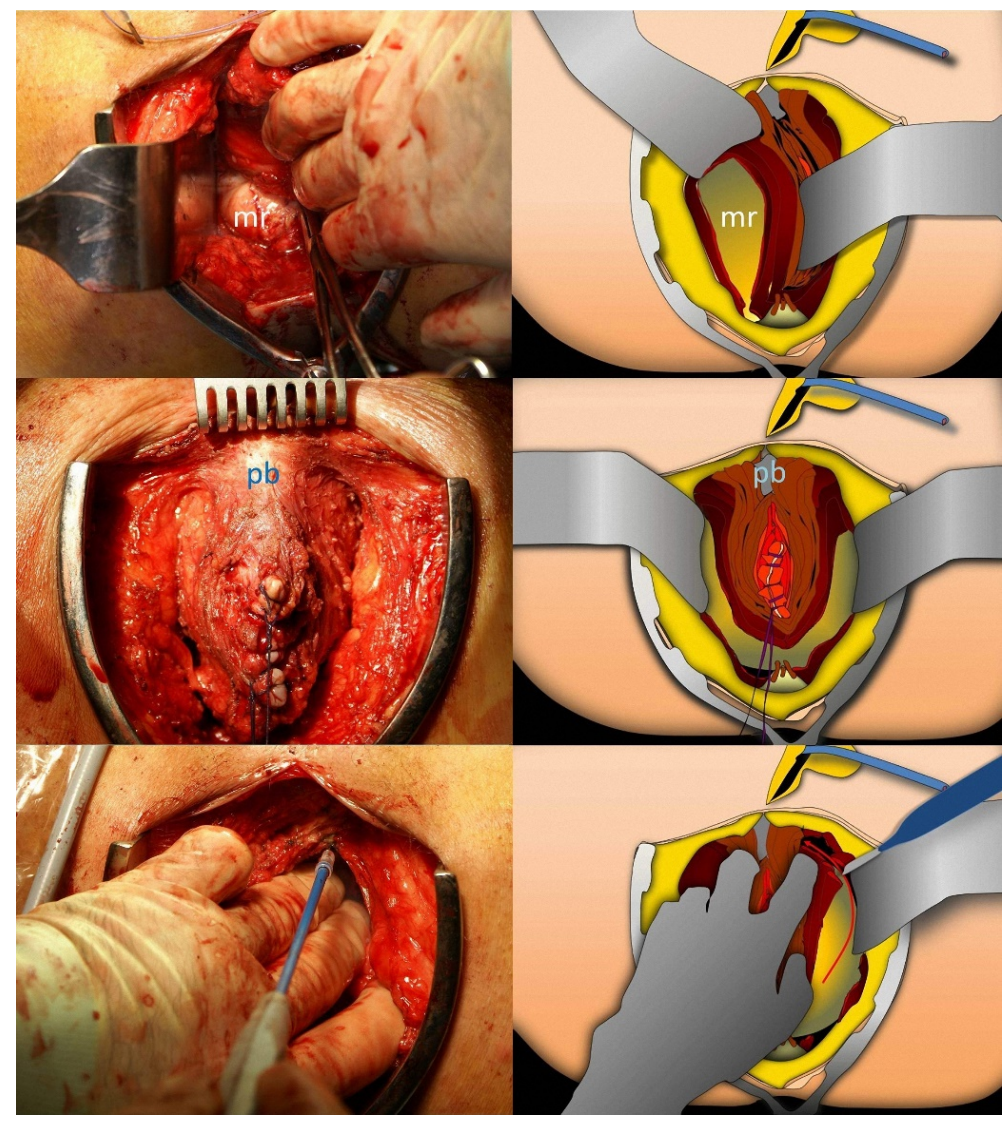

Figure 2.5 Perineal phases of an extralevator abdomino-perineal excision in supine position. (mr: mesorectum; pb perineal body) 
In both positions a complete extralevator abdomino-perineal excision can be performed. In prone position visibility of the perineal operating field is better at the cost of a wider incision, which requires closure with a (biological) mesh or musculocutaneous flap. ${ }^{9}$ In the supine position simultaneous access to the tumour from the abdomen and perineum may be an advantage in more advanced tumours. An intersphincteric or ischiorectal approach is more commonly performed in supine position. In both positions, the abdominal phase may also be performed laparoscopically.

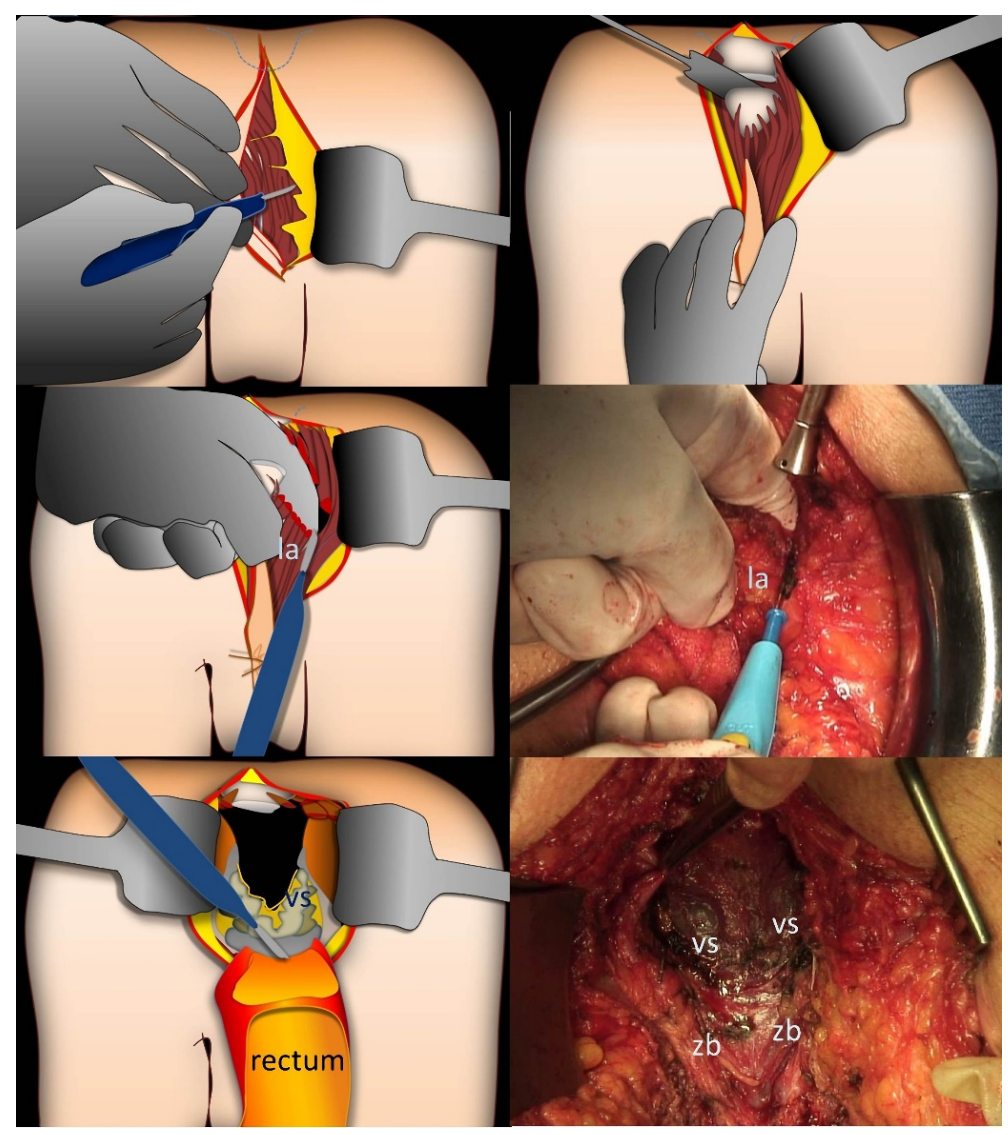

Figure 2.6 Perineal phases of an extralevator abdomino-perineal excision in prone position. (la: levator ani muscle; vs: vesiculea seminales; zb: nerve bundles of Walsh) 


\section{Future perspectives}

\section{Registry}

On a population-based level, outcome of rectal cancer treatment differs not only widely among countries, but also within countries among hospitals and even within hospitals among individual surgeons. ${ }^{18,67-69}$ But in the end, the chain of treatments given to an individual patient can be traced back to each individual link of the chain. If the quality of pathology is excellent, it will enable one to unravel the different prognostic variables, which apply to an individual patient. Not only can the biology of the tumour, but also the quality of surgery and the effect of neoadjuvant treatment be ascertained. Furthermore the anatomical information of the MRI may be linked to the outcome and quality of the surgical procedure. Registration of these variables will identify the weak links and will allow better quality of the complete chain and improving outcome and reducing the burden of treatment costs for avoidable poor results (Figure 2.7). ${ }^{70}$

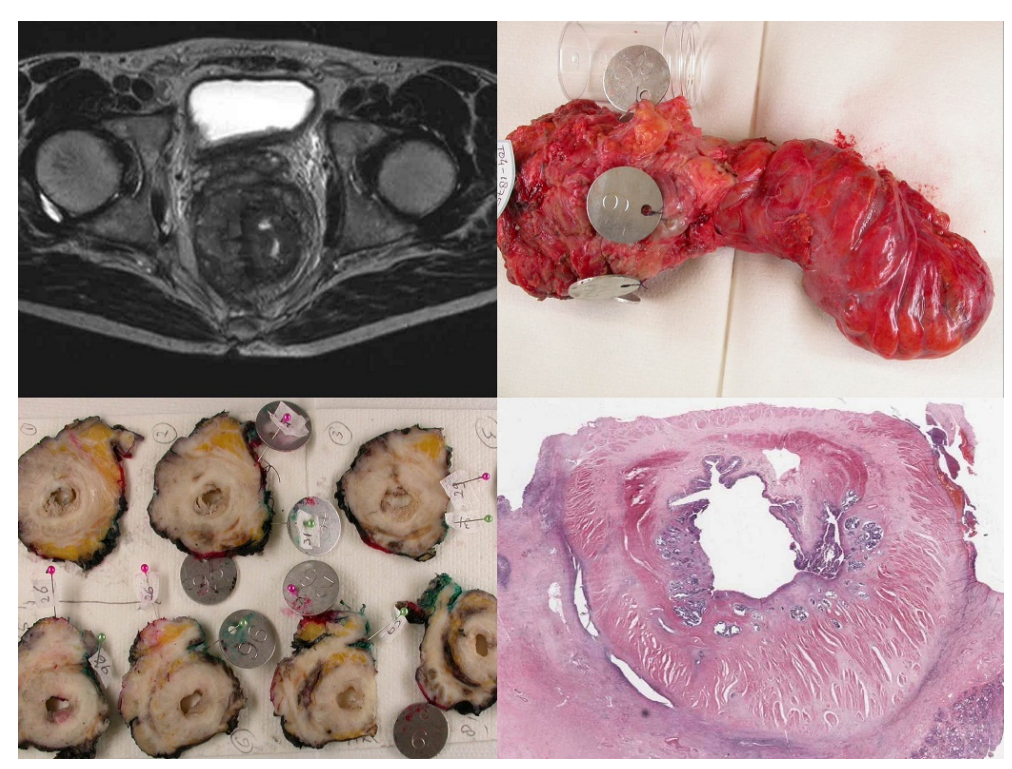

Figure 2.7 Good collaboration of radiologist, surgeon and pathologist enables continuous quality improvement. Good registration is an indispensable prerequisite (www.virtualpathology.leeds.ac.uk).

Localised rectal cancer, but also metastasized rectal cancer, must be treated by a multidisciplinary team. In order to achieve the best quality of treatment, the planning of the treatment and the sequence of the different treatment modalities have to be 
decided upon before any treatment is given. During a multidisciplinary team meeting (MDT) after results of imaging and histopathology biopsies have become available, the specific problems of a rectal cancer can be identified and the best approach for the individual patient may be selected, depending on the presence/exclusion of metastatic disease, local extent of the tumour and the patient's condition. It is important for the patient to know who has the role of the director of the treatment. In most cases this responsibility lies with the surgeon, who is not only responsible for the surgery but for all components involved in the treatment planning.

On a local level the use of standard protocols, the registration of the MDT meetings and the registration of the important outcome parameters can help to identify blind spots. These data can constitute the basis for a larger possibly national registry. ${ }^{71}$ These national registries can be used to compare countries. In Norway, Sweden, Denmark, the UK and the Netherlands mandatory registration has led to almost $100 \%$ coverage of the population.

EUROCARE collects colorectal cancer data from all European countries and was able to show large differences in outcome in Europe. ${ }^{67}$ A limitation on the overall use of the EUROCARE database is the wide spread in coverage of their populations between the European countries. It is difficult to compare results between countries with coverage of less than $50 \%$ and countries with coverage of $100 \%$. Furthermore the completeness of data on important clinical data such as stage distribution and cancer sub sites varies widely between registries. For example The United Kingdom had poorer oncological results than France and Germany; however, the UK has coverage of $100 \%$ compared to coverage of $18 \%$ and $1 \%$ respectively for France and Germany. Explaining difference in outcome between countries with different coverage is difficult, particularly when it is unclear if coverage of less than $50 \%$ is representative for the country as a whole.

But then again, there is no need to create a scale of the outcome of different countries. It is more relevant to identify the best practice and to set European guidelines based on the best knowledge available. ${ }^{72,73}$ The EURECCA colorectal project, which promotes registry based on consensus subsequent sharing of data can lead to a better outcome for rectal cancer patients all over the world. ${ }^{67,74-77}$

\section{Centralization}

With the improvement in care during the last decades and the introduction of MDT meetings, the oncological outcome of rectal cancer has greatly improved. Another more recently development and improvement in cancer care is the introduction of centralisation of care for advanced cases and major surgery. In rectal cancer this also plays an important role. In advanced cases the cancer grows through its surrounding fascia into other organs and structures. In those advanced cases, when an exenteration is needed the resection could consist of an orthopaedic, gynaecologic and urologic en bloc resection combined with the rectal resection. It is not desirable that such a 
procedure is performed by multiple surgeons. Therefore the rectal cancer surgeon has to be a complete pelvic surgeon. However, normally those advanced cases have a low incidence in a normal regional hospital. The small numbers increase the risk of performing an irradical resection as the experience with those cases is limited. Centralization of advanced rectal cancer cases will not only result in less irradical resections but also in better post-operative care. As a hospital treats more advanced cases all specialties involved in rectal cancer care gain more experience. Furthermore post-operative complications are seen sooner, radiological imaging is interpreted better by the radiologist and pathologists gain more experience with large specimens and the influence of neo-adjuvant treatment. All this will result in lower mortality rates and better oncological outcome. Specialisation has led to improved outcome in rectal cancer treatment, how much more this will be true for locally advanced cases requiring more individualized surgery. ${ }^{78-80}$

\section{Patient reported outcome measures}

Due to the major improvements in therapy and oncological outcome in the last decades, the influence of treatment on the individual patient has become more and more important. Particular in the last decade, where outcome of a disease is not the only measurement of adequate treatment, there is an increasing interest in the influence of treatment on patients' health-related quality of life (HRQL). Most of the studies publishing on HRQL and rectal cancer use generally used questionnaires such as the SF-36, EORTC QLQ-C30 OR EORTC QLQ-C30. These questionnaires are reliable, valid and responsive, but have not been developed to assess treatment on an individual level. ${ }^{81-83}$

The modern rectal cancer patient is confronted with a combination of treatments, which all to a certain extent will influence his way of living. The patient will probably like to be informed about alternatives in their treatment schedule and about consequences of their choice. It is important for future research to focus on different and more interesting patient groups, such as frail patients and elderly. In these patients, it is more likely that the assumed benefits of survival give an increase in morbidity and could have an adverse effect on HRQL. Future studies on rectal cancer have not only to focus at the effect of additional treatment on survival, but also the influence of the treatment on quality of life. If the treatment results in an increase in survival, is it the possible decrease in HRQL worthwhile? It is likely that those studies will make the decision process for the involved specialties in the treatment of rectal cancer easier and will result in a more patient tailored treatment. 


\section{Conclusion}

Surgery is still the cornerstone of rectal cancer treatment for the time being. Therefore, the surgeon should take the role of the director of the treatment plan and he should realize that surgery is an integral part of a comprehensive multidisciplinary approach. The basis for quality assurance is registration. A modern anatomical surgical approach requires also a modern attitude towards quality assurance and an obligation to keep the patient well-informed about the choices which have been made and to allow the patient to have his/her own say in the matter. 


\section{References}

1. Lange MM, Rutten HJ, van de Velde CJ. One hundred years of curative surgery for rectal cancer: 19082008. Eur J Surg Oncol 2009;35:456-63.

2. Heald RJ, Ryall RD. Recurrence and survival after total mesorectal excision for rectal cancer. Lancet 1986;1:1479-82.

3. Quirke P, Durdey P, Dixon MF, Williams NS. Local recurrence of rectal adenocarcinoma due to inadequate surgical resection. Histopathological study of lateral tumour spread and surgical excision. Lancet 1986;2:996-9.

4. den Dulk M, Marijnen CA, Putter $\mathrm{H}$, et al. Risk factors for adverse outcome in patients with rectal cancer treated with an abdominoperineal resection in the total mesorectal excision trial. Ann Surg 2007;246: 83-90.

5. den Dulk M, Putter $\mathrm{H}$, Collette $\mathrm{L}$, et al. The abdominoperineal resection itself is associated with an adverse outcome: the European experience based on a pooled analysis of five European randomised clinical trials on rectal cancer. Eur J Cancer 2009;45:1175-83.

6. Marr R, Birbeck K, Garvican J, et al. The modern abdominoperineal excision: the next challenge after total mesorectal excision. Ann Surg 2005;242:74-82.

7. Nagtegaal ID, van de Velde CJ, Marijnen CA, van Krieken JH, Quirke P. Low rectal cancer: a call for a change of approach in abdominoperineal resection. J Clin Oncol 2005;23:9257-64.

8. How $P$, Shihab $O$, Tekkis $P$, et al. A systematic review of cancer related patient outcomes after anterior resection and abdominoperineal excision for rectal cancer in the total mesorectal excision era. Surg Oncol 2011;20:e149-55.

9. Holm T, Ljung A, Haggmark T, Jurell G, Lagergren J. Extended abdominoperineal resection with gluteus maximus flap reconstruction of the pelvic floor for rectal cancer. Br J Surg 2007;94:232-8.

10. Martijnse IS, Dudink RL, West NP, et al. Focus on extralevator perineal dissection in supine position for low rectal cancer has led to better quality of surgery and oncologic outcome. Ann Surg Oncol 2012;19: 786-93.

11. West NP, Finan PJ, Anderin C, Lindholm J, Holm T, Quirke P. Evidence of the oncologic superiority of cylindrical abdominoperineal excision for low rectal cancer. J Clin Oncol 2008;26:3517-22.

12. West NP, Anderin C, Smith KJ, Holm T, Quirke P. Multicentre experience with extralevator abdominoperineal excision for low rectal cancer. Br J Surg 2010:97:588-99.

13. Beets-Tan RG, Beets GL, Vliegen RF, et al. Accuracy of magnetic resonance imaging in prediction of tumour-free resection margin in rectal cancer surgery. Lancet 2001;357:497-504.

14. Brown G, Radcliffe AG, Newcombe RG, Dallimore NS, Bourne MW, Williams GT. Preoperative assessment of prognostic factors in rectal cancer using high-resolution magnetic resonance imaging. $\mathrm{Br}$ J Surg 2003;90:355-64.

15. Radcliffe A, Brown G. Will MRI provide maps of lines of excision for rectal cancer? Lancet 2001;357: 495-6.

16. Nagtegaal ID, van de Velde CJ, van der Worp E, Kapiteijn E, Quirke P, van Krieken JH. Macroscopic evaluation of rectal cancer resection specimen: clinical significance of the pathologist in quality control. J Clin Oncol 2002;20:1729-34.

17. Nagtegaal ID, Quirke P. What is the role for the circumferential margin in the modern treatment of rectal cancer? J Clin Oncol 2008;26:303-12.

18. Birbeck KF, Macklin CP, Tiffin NJ, et al. Rates of circumferential resection margin involvement vary between surgeons and predict outcomes in rectal cancer surgery. Ann Surg 2002;235:449-57.

19. Martijnse I, West N, Quirke P, Heald R, van de Velde C, Rutten HJ. Will extralevator abdominoperineal excision become the new gold standard? In Valentini V, Schmoll H-J, van de Velde C, eds. Multidisciplinary management of Rectal cancer. 1 ed. New York, Springer 2012:261-274.

20. Quirke P, Steele R, Monson J, et al. Effect of the plane of surgery achieved on local recurrence in patients with operable rectal cancer: a prospective study using data from the MRC CR07 and NCIC-CTG CO16 randomised clinical trial. Lancet 2009;373:821-8.

21. Vliegen $R$, Dresen R, Beets $G$, et al. The accuracy of Multi-detector row CT for the assessment of tumor invasion of the mesorectal fascia in primary rectal cancer. Abdom Imaging 2008;33:604-10. 
22. Wolberink SV, Beets-Tan RG, Nagtegaal ID, Wiggers T. Preoperative assessment of the circumferential margin in rectal cancer is more informative in treatment planning than the T stage. Tech Coloproctol 2006;10:171-6.

23. Maizlin ZV, Brown JA, So G, et al. Can CT replace MRI in preoperative assessment of the circumferential resection margin in rectal cancer? Dis Colon Rectum 2010;53:308-14.

24. Doornebosch PG, Bronkhorst PJ, Hop WC, Bode WA, Sing AK, de Graaf EJ. The role of endorectal ultrasound in therapeutic decision-making for local vs. transabdominal resection of rectal tumors. Dis Colon Rectum 2008;51:38-42.

25. Extramural depth of tumor invasion at thin-section MR in patients with rectal cancer: results of the MERCURY study. Radiology 2007;243:132-9.

26. Merkel S, Mansmann U, Siassi M, Papadopoulos T, Hohenberger W, Hermanek P. The prognostic inhomogeneity in pT3 rectal carcinomas. Int J Colorectal Dis 2001;16:298-304.

27. Taylor FG, Quirke P, Heald RJ, et al. One millimetre is the safe cut-off for magnetic resonance imaging prediction of surgical margin status in rectal cancer. Br J Surg 2011;98:872-9.

28. Ptok H, Ruppert R, Stassburg J, et al. Pretherapeutic MRI for decision-making regarding selective neoadjuvant radiochemotherapy for rectal carcinoma: Interim analysis of a multicentric prospective observational study. J Magn Reson Imaging 2013;37:1122-8.

29. Bipat S, Glas AS, Slors FJ, Zwinderman AH, Bossuyt PM, Stoker J. Rectal cancer: local staging and assessment of lymph node involvement with endoluminal US, CT, and MR imaging--a meta-analysis. Radiology 2004;232:773-83.

30. Lahaye MJ, Engelen SM, Nelemans PJ, et al. Imaging for predicting the risk factors--the circumferential resection margin and nodal disease--of local recurrence in rectal cancer: a meta-analysis. Semin Ultrasound CT MR 2005;26:259-68.

31. Kim JH, Beets GL, Kim MJ, Kessels AG, Beets-Tan RG. High-resolution MR imaging for nodal staging in rectal cancer: are there any criteria in addition to the size? Eur J Radiol 2004;52:78-83.

32. Kotanagi H, Fukuoka T, Shibata $Y$, et al. The size of regional lymph nodes does not correlate with the presence or absence of metastasis in lymph nodes in rectal cancer. J Surg Oncol 1993;54:252-4.

33. Koh DM, Brown G, Temple L, et al. Rectal cancer: mesorectal lymph nodes at MR imaging with USPIO versus histopathologic findings--initial observations. Radiology 2004;231:91-9.

34. Lahaye MJ, Engelen SM, Kessels AG, et al. USPIO-enhanced MR imaging for nodal staging in patients with primary rectal cancer: predictive criteria. Radiology 2008;246:804-11.

35. Lambregts DM, Beets GL, Maas M, et al. Accuracy of gadofosveset-enhanced MRI for nodal staging and restaging in rectal cancer. Ann Surg 2011;253:539-45.

36. Lambregts DM, Beets GL, Maas M, et al. Tumour ADC measurements in rectal cancer: effect of ROI methods on ADC values and interobserver variability. Eur Radiol 2011;21:2567-74.

37. Brown G, Richards CJ, Bourne MW, et al. Morphologic predictors of lymph node status in rectal cancer with use of high-spatial-resolution MR imaging with histopathologic comparison. Radiology 2003; 227:371-7.

38. Patel UB, Taylor F, Blomqvist L, et al. Magnetic resonance imaging-detected tumor response for locally advanced rectal cancer predicts survival outcomes: MERCURY experience. J Clin Oncol 2011;29: 3753-60.

39. Patel UB, Brown G, Rutten $\mathrm{H}$, et al. Comparison of magnetic resonance imaging and histopathological response to chemoradiotherapy in locally advanced rectal cancer. Ann Surg Oncol 2012;19:2842-52.

40. Vliegen RF, Beets GL, Lammering G, et al. Mesorectal fascia invasion after neoadjuvant chemotherapy and radiation therapy for locally advanced rectal cancer: accuracy of MR imaging for prediction. Radiology 2008;246:454-62.

41. Patel UB, Taylor F, Blomqvist L, et al. Magnetic resonance imaging-detected tumor response for locally advanced rectal cancer predicts survival outcomes: MERCURY experience. J Clin Oncol 2011;29: 3753-60.

42. Patel UB, Blomqvist LK, Taylor F, et al. MRI after treatment of locally advanced rectal cancer: how to report tumor response--the MERCURY experience. AJR Am J Roentgenol 2012;199:W486-95.

43. Lahaye MJ, Beets GL, Engelen SM, et al. Locally advanced rectal cancer: MR imaging for restaging after neoadjuvant radiation therapy with concomitant chemotherapy. Part II. What are the criteria to predict involved lymph nodes? Radiology 2009;252:81-91. 
44. Barbaro B, Fiorucci C, Tebala C, et al. Locally advanced rectal cancer: MR imaging in prediction of response after preoperative chemotherapy and radiation therapy. Radiology 2009;250:730-9.

45. Barbaro B, Vitale R, Leccisotti L, et al. Restaging locally advanced rectal cancer with MR imaging after chemoradiation therapy. Radiographics 2010;30:699-716.

46. Kusters $\mathrm{M}$, Beets $\mathrm{GL}$, van de Velde $\mathrm{CJ}$, et al. A comparison between the treatment of low rectal cancer in Japan and the Netherlands, focusing on the patterns of local recurrence. Ann Surg 2009;249:229-35.

47. Folkesson J, Birgisson H, Pahlman L, Cedermark B, Glimelius B, Gunnarsson U. Swedish Rectal Cancer Trial: long lasting benefits from radiotherapy on survival and local recurrence rate. J Clin Oncol 2005;23: 5644-50.

48. Sebag-Montefiore D, Stephens RJ, Steele R, et al. Preoperative radiotherapy versus selective postoperative chemoradiotherapy in patients with rectal cancer (MRC CR07 and NCIC-CTG C016): a multicentre, randomised trial. Lancet 2009;373:811-20.

49. van GW, Marijnen CA, Nagtegaal ID, et al. Preoperative radiotherapy combined with total mesorectal excision for resectable rectal cancer: 12-year follow-up of the multicentre, randomised controlled TME trial. Lancet Oncol 2011;12:575-82.

50. Martling A, Holm T, Johansson H, Rutqvist LE, Cedermark B. The Stockholm II trial on preoperative radiotherapy in rectal carcinoma: long-term follow-up of a population-based study. Cancer 2001;92: 896-902.

51. Pettersson D, Glimelius B, Iversen H, Johansson H, Holm T, Martling A. Impaired postoperative leucocyte counts after preoperative radiotherapy for rectal cancer in the Stockholm III Trial. Br J Surg 2013;100:969-975.

52. Sauer R, Liersch T, Merkel S, et al. Preoperative versus postoperative chemoradiotherapy for locally advanced rectal cancer: results of the German CAO/ARO/AIO-94 randomized phase III trial after a median follow-up of 11 years. J Clin Oncol 2012;30:1926-33.

53. Bosset JF, Calais G, Mineur L, et al. Enhanced tumorocidal effect of chemotherapy with preoperative radiotherapy for rectal cancer: preliminary results--EORTC 22921. J Clin Oncol 2005;23:5620-7.

54. Valentini V, van Stiphout RG, Lammering G, et al. Nomograms for predicting local recurrence, distant metastases, and overall survival for patients with locally advanced rectal cancer on the basis of European randomized clinical trials. J Clin Oncol 2011;29:3163-72.

55. Bujko K, Nowacki MP, Nasierowska-Guttmejer A, Michalski W, Bebenek M, Kryj M. Long-term results of a randomized trial comparing preoperative short-course radiotherapy with preoperative conventionally fractionated chemoradiation for rectal cancer. Br J Surg 2006;93:1215-23.

56. Bujko K, Kepka L, Michalski W, Nowacki MP. Does rectal cancer shrinkage induced by preoperative radio(chemo)therapy increase the likelihood of anterior resection? A systematic review of randomised trials. Radiother Oncol 2006;80:4-12.

57. van Dijk TH, Tamas K, Beukema JC, et al. Evaluation of short-course radiotherapy followed by neoadjuvant bevacizumab, capecitabine, and oxaliplatin and subsequent radical surgical treatment in primary stage IV rectal cancer. Ann Oncol 2013;24:1762-9.

58. RAPIDO. 2013. http://www.dccg.nl/node/99

59. Law WL, Chu KW. Anterior resection for rectal cancer with mesorectal excision: a prospective evaluation of 622 patients. Ann Surg 2004;240:260-8.

60. Wallner C, Lange MM, Bonsing BA, et al. Causes of fecal and urinary incontinence after total mesorectal excision for rectal cancer based on cadaveric surgery: a study from the Cooperative Clinical Investigators of the Dutch total mesorectal excision trial. J Clin Oncol 2008;26:4466-72.

61. Lindsey I, Guy RJ, Warren BF, Mortensen NJ. Anatomy of Denonvilliers' fascia and pelvic nerves, impotence, and implications for the colorectal surgeon. Br J Surg 2000;87:1288-99.

62. Lindsey I, Warren BF, Mortensen NJ. Denonvilliers' fascia lies anterior to the fascia propria and rectal dissection plane in total mesorectal excision. Dis Colon Rectum 2005;48:37-42.

63. Stelzner S, Holm T, Moran BJ, et al. Deep pelvic anatomy revisited for a description of crucial steps in extralevator abdominoperineal excision for rectal cancer. Dis Colon Rectum 2011;54:947-57.

64. Stelzner S, Koehler C, Stelzer J, Sims A, Witzigmann H. Extended abdominoperineal excision vs. standard abdominoperineal excision in rectal cancer--a systematic overview. Int J Colorectal Dis 2011;26: 1227-40. 
65. Holm T. Abdominoperineal resection revisited: is positioning an important issue? Dis Colon Rectum 2011;54:921-2.

66. Shihab OC, Heald RJ, Holm T, et al. A pictorial description of extralevator abdominoperineal excision for low rectal cancer. Colorectal Dis 2012;14:e655-60.

67. Brenner $\mathrm{H}$, Bouvier AM, Foschi $\mathrm{R}$, et al. Progress in colorectal cancer survival in Europe from the late 1980s to the early 21st century: the EUROCARE study. Int J Cancer 2012;131:1649-58.

68. Porter GA, Soskolne CL, Yakimets WW, Newman SC. Surgeon-related factors and outcome in rectal cancer. Ann Surg 1998;227:157-67.

69. Richardson DP, Porter GA, Johnson PM. Surgeon knowledge contributes to the relationship between surgeon volume and patient outcomes in rectal cancer. Ann Surg 2013;257:295-301.

70. Pahlman L, Bohe M, Cedermark B, et al. The Swedish rectal cancer registry. Br J Surg 2007;94:1285-92.

71. van GW, van de Velde CJ. Improving quality of cancer care through surgical audit. Eur J Surg Oncol 2010;36 Suppl 1:S23-6.

72. Taylor I, Van d, V, van GW. A consensus approach to rectal cancer management. Eur J Surg Oncol 2010;36:111-3.

73. van GW, Wouters MW, Peeters KC, van de Velde CJ. Nationwide outcome registrations to improve quality of care in rectal surgery. An initiative of the European Society of Surgical Oncology. J Surg Oncol 2009;,99:491-6.

74. Mroczkowski P, Ortiz H, Penninckx F, Pahlman L. European quality assurance programme in rectal cancer--are we ready to launch? Colorectal Dis 2012;14:960-6.

75. Valentini V, Aristei C, Glimelius B, et al. Multidisciplinary Rectal Cancer Management: 2nd European Rectal Cancer Consensus Conference (EURECA-CC2). Radiother Oncol 2009;92:148-63.

76. Valentini V, van Stiphout RG, Lammering G, et al. Nomograms for predicting local recurrence, distant metastases, and overall survival for patients with locally advanced rectal cancer on the basis of European randomized clinical trials. J Clin Oncol 2011;29:3163-72.

77. van GW, van den Broek CB, Mroczkowski P, et al. The EURECCA project: Data items scored by European colorectal cancer audit registries. Eur J Surg Oncol 2012;38:467-71.

78. Borowski DW, Kelly SB, Bradburn DM, Wilson RG, Gunn A, Ratcliffe AA. Impact of surgeon volume and specialization on short-term outcomes in colorectal cancer surgery. Br J Surg 2007;94:880-9.

79. Borowski DW, Bradburn DM, Mills SJ, et al. Volume-outcome analysis of colorectal cancer-related outcomes. Br J Surg 2010;97:1416-30.

80. McArdle CS, Hole DJ. Influence of volume and specialization on survival following surgery for colorectal cancer. Br J Surg 2004;91:610-7.

81. Orsini RG, Thong MS, van de Poll-Franse LV, et al. Quality of life of older rectal cancer patients is not impaired by a permanent stoma. Eur J Surg Oncol 2013;39:164-70.

82. Rauch P, Miny J, Conroy T, Neyton L, Guillemin F. Quality of life among disease-free survivors of rectal cancer. J Clin Oncol 2004;22:354-60.

83. Vironen $\mathrm{JH}$, Kairaluoma M, Aalto AM, Kellokumpu IH. Impact of functional results on quality of life after rectal cancer surgery. Dis Colon Rectum 2006;49:568-78. 

Age related differences in

PART I outcome of rectal cancer

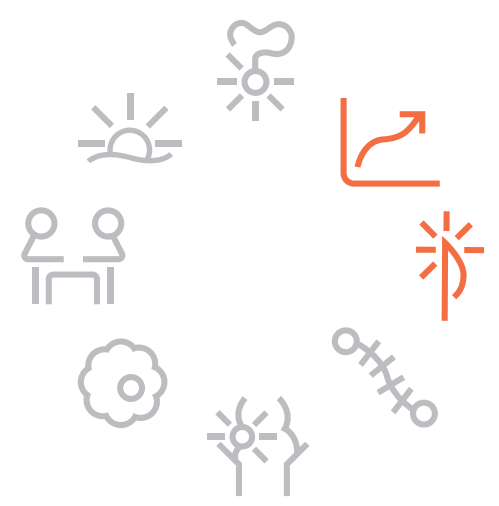



CHAPTER 3 Comparable survival for young rectal cancer patients, despite unfavourable morphology and more advanced-stage disease

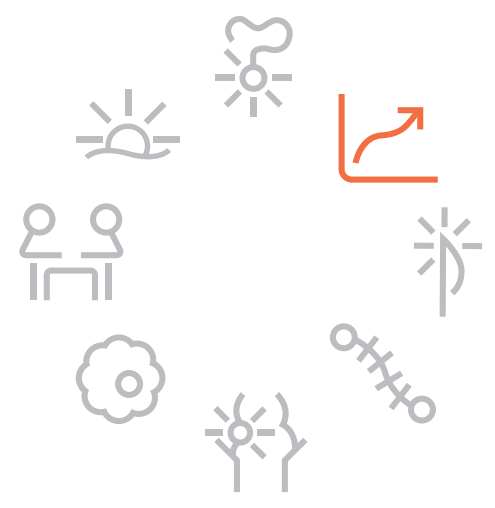

R.G. Orsini

R.H. Verhoeven

V.E. Lemmens

L.N. van Steenbergen

I.H. de Hingh

G.A. Nieuwenhuijzen

H.J. Rutten

Eur J Cancer 


\section{Abstract}

\section{Background}

Young patients with rectal cancer tend to present with more advanced-stage disease and unfavourable tumour morphology. The effects of these tumour characteristics on survival in this particular patient group are unclear.

\section{Methods}

Population-based data from the Netherlands Cancer Registry (NCR) were used. Data from patients diagnosed with rectal cancer between 1989 and 2010 were selected. Younger patients ( $\leq 40$ years) were compared with middle-aged patients (41-70 years) with respect to disease stage, tumour characteristics, treatment and outcomes. Patients aged older than 70 years were excluded. Relative excess risk (RER) models were used to perform uni- and multivariate survival analyses.

\section{Results}

A total of 37.056 patients were included ( $\leq 40$ years $n=1.102$ ). Compared with middleaged patients, young patients were more likely to have stage III (33,8\% vs. $27,8 \%)$ and stage IV $(24,3 \%$ vs. $19,6 \%)$ disease $(p<0,001)$. Young patients also presented more frequently with mucinous tumours $(10,8 \%$ vs. $9,0 \%)$, signet cell carcinomas $(2,6 \%$ vs. $0,6 \%)$ and poorly differentiated tumours $(16,6 \%$ vs. $12,3 \%)(p=0,001)$. The treatment of stage I-III patients did not differ between the two groups, except regarding adjuvant chemotherapy, which was more often given to young patients $(24,3 \%$ vs. $14,4 \%$, $p<0,001)$. Young age was a prognostic factor for better survival in stage I-III patients (RER 0,82 Cl 0,71-0,94). Adjuvant chemotherapy was associated with improved survival in stage I-III patients (RER $0,76,95 \% \mathrm{Cl} 0,70-0,83$ ). In an exploratory analysis, adjuvant chemotherapy in young stage III and pN1 patients was associated with improved survival.

\section{Conclusions}

Young patients present with more advanced disease and have more unfavourable tumour characteristics compared with middle-aged patients. Despite these characteristics, survival rates are equal, and young age is a prognostic factor for better survival. Although the use of adjuvant chemotherapy is controversial, a positive correlation with survival was found in this study. 


\section{Introduction}

Colorectal cancer is the second most common malignancy worldwide. ${ }^{1}$ In the Netherlands, it is the third most frequent type of cancer. Approximately one-third of patients with a colorectal malignancy have rectal carcinoma. In 2013, almost 4000 new cases of rectal cancer were diagnosed in the Netherlands. ${ }^{2}$ Rectal cancer mainly affects middle-aged and elderly individuals, with more than $75 \%$ of patients being diagnosed at 60 years or older. ${ }^{2,3}$ However, during the last several decades, an increasing incidence of patients diagnosed with rectal cancer at the age of 40 years or younger has been observed. $^{4,5}$

Young patients with rectal cancer more often present with advanced-stage disease and unfavourable tumour morphology compared with middle-aged patients. ${ }^{6-10}$ Studies focusing on survival in young rectal cancer patients have shown at least equal or better outcomes compared with older patients. ${ }^{9,10}$ However, other studies have found contradictory survival rates when young patients present with more advanced disease. $^{6,11}$

Overall, the assumption that young patients with rectal cancer have poorer survival is mainly based on studies performed prior to or during the introduction of total mesorectal excision (TME) surgery and neo-adjuvant treatment schemes with radiation and/or chemotherapy. ${ }^{11,12}$ More recent studies have been smaller and included patients with both colon and rectal cancer, leading to uncertainty about the possible effects of adverse tumour morphology and advanced-stage disease on survival in young rectal cancer patients. $6,8,10,13,14$

For stage III colon cancer patients, there is a significant survival benefit from adjuvant chemotherapy, regardless of age. ${ }^{15,16}$ However, for stage III rectal cancer, there is a lack of consensus regarding adjuvant treatment amongst multidisciplinary experts and in international guidelines. ${ }^{17,18}$ Additionally, no distinctions are made regarding age, likely due to the lack of information about the effect of adjuvant chemotherapy in young rectal cancer patients. In randomised controlled trials only a minority of the patients can be considered young ( $\leq 40$ years). In the most recent meta-analysis about adjuvant chemotherapy after pre-operative (chemo-)radiotherapy in rectal cancer less than $2 \%$ of the 1196 included patients were under the age of 45 years. ${ }^{19}$ To obtain further insights concerning the influence of adjuvant chemotherapy on survival in young patients, large population-based studies may be performed, but tumour and treatment characteristics are not always available in these databases.

In both younger and middle-aged patients, life expectancy is good and is less confounded by other variables, such as co-morbidities or age-dependent physiological 
decline, unlike in elderly patients. Therefore, this study was conducted to investigate the differences in tumour stage, morphology, treatment and survival between young (aged $\leq 40$ years) and middle-aged (41-70 years) rectal cancer patients in a large, nationwide population-based database of patients diagnosed with rectal cancer in the Netherlands. In a secondary analysis, the influence of adjuvant chemotherapy on survival was investigated only in young patients because these patients are the least likely to be affected by confounding variables.

\section{Methods}

For this study, population-based data from the Netherlands Cancer Registry (NCR) were used. The NCR has collected data on all newly diagnosed patients with cancer in the Netherlands since 1989. Registration is primarily based on notification by the automated pathology archive (PALGA) and hospital discharge notes. Clinical and demographic information, tumour characteristics and primary treatment are routinely extracted from medical records by specially trained administrators of the cancer registry. The vital status of all patients is assessed through annual linkage with Municipal Administrative Databases, which register all deceased and emigrated persons in the Netherlands.

We selected all patients who were diagnosed with rectal cancer (ICD-O codes C19 and C20) and registered by the NCR between 1989 and 2010. Tumour characteristics were coded using the International Classification of Disease for Oncology 3th Edition tool (ICD-O3). Patients with tumours other than adenocarcinomas, mucinous carcinomas or signet cell carcinomas were excluded. For this study, the patient population was divided into two groups: young rectal cancer patients, aged $\leq 40$ years, and middle-aged rectal cancer patients, aged 41-70 years. Patients older than 70 years were excluded from the analysis because of the completely different dynamics regarding survival, ageing and the influence of comorbidities in these patients.

Differences in clinical and demographic parameters between groups were compared using chi-square or t-tests where appropriate. When normality and homogeneity assumptions were violated, non-parametric tests were used. Surgery as a variable was only used if there was a resection of the primary tumor. Those patients who had only received a stoma or other palliative surgery were not coded as receiving surgery. The stage of disease was assigned using the clinical TNM stage; if the clinical TNM stage was missing, the pathological TNM stage was used in this calculation.

All patients were divided a priori into three groups based on the year of diagnosis: 1989-1994 (prior to the introduction of neo-adjuvant treatment and TME surgery), 
1995-2000 (during the introduction of TME surgery in the Netherlands), and 2001-2010 (after the implementation of TME surgery and the introduction of the first Dutch national rectal cancer treatment guidelines). For all analyses of survival, we used relative survival. Relative survival is an estimation of disease-specific survival. It is calculated as the absolute survival amongst cancer patients divided by the expected survival for the general population with the same sex and age structure. Traditional cohort-based relative survival analysis according to the Ederer II method was used to calculate 5-year relative survival. ${ }^{20}$ Survival between the age groups was subjected to uni- and multivariate testing using a multiple relative survival regression model to calculate the variable specific relative excess risk (RER) of the death estimates.

In the secondary analysis, the influence of adjuvant chemotherapy on survival was calculated in patients aged $\leq 40$ years. The RER scores based on adjuvant chemotherapy for all variables shown in the multiple relative survival regression model were calculated in a univariate manner in patients aged $\leq 40$ years with stage I-III rectal cancer.

\section{Results}

\section{Patient and tumour characteristics}

A total of 66,581 patients with primary rectal cancer were registered by the NCR between 1989 and 2010. Patients older than 70 years were excluded, resulting in a total of 37.056 patients. The young age group ( $\leq 40$ years) consisted of 1.102 (3\%) patients, and the middle-aged group included 35.954 patients (97\%). The median age at the time of diagnosis in the young group was 35.1 years, compared with 60.2 years in the middle-aged group. There were fewer males in the young age group compared with the middle-aged group ( $53 \%$ vs. $62 \%$, p<0.001). (Table 3.1)

Compared with middle-aged patients, young patients presented more often with stage III ( $34 \%$ vs. $28 \%)$ and stage IV ( $24 \%$ vs. $20 \%)$ disease, whereas middle-aged patients presented more often with less advanced cancers (stage I-II). Younger patients were also more likely to have mucinous type carcinomas (10.8\% vs. $9.0 \%)$ and signet cell carcinomas $(2.6 \%$ vs. $0.6 \%)(p<0.001)$ than middle-aged patients. Younger patients were also more likely to have poorly (G3) or moderately (G2) differentiated tumours than middle-aged patients $(16.6 \%$ vs. $12.3 \%$ and $53.1 \%$ vs. $56.6 \%$, respectively, $p=0.001$ ).

No differences were observed in terms of the use of neo-adjuvant radiotherapy and chemoradiation between young and middle-aged rectal cancer patients. Overall, young patients were less likely to undergo surgery $(85.9 \%$ vs. $88.5 \%, p=0.008)$ but more likely to receive adjuvant chemotherapy $(23.6 \%$ vs. $14.3 \%, p<0.001)$ compared with middle- 
aged patients. However, both groups of patients with local disease (stage I-III) were equally likely to undergo surgery $(97.4 \%$ vs. $97.1 \% \mathrm{p}=0.7)$. In patients with metastatic disease (stage IV) patients were less likely to undergo surgery $(50.4 \%$ vs. $53.5 \% p=0.3)$ compared to stage I-III disease. No differences were observed between the two age groups regarding treatment in stage IV disease, with the exception of chemotherapy; younger patients received chemotherapy more often $(69.8 \%$ vs. $54.2 \%, p<0.001$; data not shown).

Table 3.1 Demographic, clinical and tumor characteristics of rectal cancer patients $\leq 70$ years in the Netherlands between 1989 and 2010.

\begin{tabular}{|c|c|c|c|}
\hline & $\begin{array}{c}\leq 40 \text { years } \\
n=1102\end{array}$ & $\begin{array}{c}1-70 \text { years } \\
n=35954\end{array}$ & $p$-value \\
\hline Age in years at time of diagnosis (mean $\pm S D$ ) & $35.1(4.7)$ & $60.2(7.2)$ & \\
\hline \multirow[t]{2}{*}{ Follow-up in years (mean $\pm S D$ ) } & $6.6(6.3)$ & $5.9(5.9)$ & 0.027 \\
\hline & $\mathrm{N}(\%)$ & $\mathrm{N}(\%)$ & \\
\hline Male & $580(52.6)$ & $22209(61.8)$ & $<0.0001$ \\
\hline \multicolumn{4}{|l|}{ Follow-up status } \\
\hline Alive & $578(52.5)$ & $17144(47.7)$ & \\
\hline Deceased & $504(45.7)$ & 18651 (51.9) & \\
\hline Emigrated & $20(1.8)$ & $159(0.4)$ & $<0.0001$ \\
\hline \multicolumn{4}{|l|}{ Tumorstage } \\
\hline I & $237(21.5)$ & $9887(27.5)$ & \\
\hline II & $224(20.3)$ & $9035(25.1)$ & \\
\hline III & $373(33.8)$ & $9978(27.8)$ & \\
\hline IV & $268(24.3)$ & 7033 (19.6) & \\
\hline Missing & - & $21(0.1)$ & $<0.0001$ \\
\hline \multicolumn{4}{|l|}{ Neoadjuvant treatment } \\
\hline Radiotherapy & $322(31.2)$ & $10894(31.9)$ & 0.44 \\
\hline Radiochemotherapy & $69(6.3)$ & $1790(5.0)$ & 0.055 \\
\hline Surgery on primary tumour & $947(85.9)$ & $31828(88.5)$ & 0.008 \\
\hline Adjuvant chemotherapy & $268(24.3)$ & $5176(14.4)$ & $<0.0001$ \\
\hline Palliative chemotherapy & $105(9.5)$ & $2092(5.8)$ & $<0.0001$ \\
\hline \multicolumn{4}{|l|}{ Morphology } \\
\hline Adenocarcinoma & $954(86.6)$ & $32510(90.4)$ & \\
\hline Mucinous carcinoma & $119(10.8)$ & $3218(9.0)$ & \\
\hline Signet cell carcinoma & $29(2.6)$ & $226(0.6)$ & $<0.0001$ \\
\hline \multicolumn{4}{|l|}{ Histopathological grade of differentation } \\
\hline Well differentiated (G1) & $72(6.5)$ & $2364(6.6)$ & \\
\hline Moderately differentiated (G2) & $585(53.1)$ & $20335(56.6)$ & \\
\hline Poorly differentiated (G3) & $183(16.6)$ & $4409(12.3)$ & \\
\hline Undifferentiated (G4) & $1(0.1)$ & $38(0.1)$ & \\
\hline Missing & $261(23.7)$ & $8808(24.5)$ & 0.001 \\
\hline \multicolumn{4}{|l|}{ Period of diagnosis } \\
\hline 1989-1994 & $269(24.4)$ & $7630(21.2)$ & \\
\hline $1995-2000$ & $281(25.5)$ & 8940 (24.9) & \\
\hline $2001-2010$ & $552(50.1)$ & $19384(53.9)$ & $<0.017$ \\
\hline
\end{tabular}




\section{Trends over time in stage distribution and relative survival}

While the percentage of young and middle-aged patients with stage I or III rectal cancer remained more or less similar over time, the percentage of patients with stage II rectal cancer decreased over time, and the percentage of patients with stage IV rectal cancer increased over time for both age groups. (Table 3.2) These changes were more pronounced in the young than in the middle-aged patients. In both patient groups, the relative survival rates improved over time. (Table 3.3)

Table 3.2 Stage distribution of rectal cancer patients in the Netherlands aged $\leq 70$ years between different time periods and according to age group.

\begin{tabular}{|c|c|c|c|c|c|c|}
\hline & \multicolumn{2}{|c|}{ 1989-1994 } & \multicolumn{2}{|c|}{$1995-2000$} & \multicolumn{2}{|c|}{ 2001-2010 } \\
\hline & $\begin{array}{c}\leq 40 \text { years } \\
\mathrm{N}=269\end{array}$ & $\begin{array}{c}41-70 \text { years } \\
\mathrm{N}=7630\end{array}$ & $\begin{array}{c}\leq 40 \text { years } \\
\mathrm{N}=281\end{array}$ & $\begin{array}{c}41-70 \text { years } \\
\mathrm{N}=8940\end{array}$ & $\begin{array}{c}\leq 40 \text { years } \\
N=552\end{array}$ & $\begin{array}{c}41-70 \text { years } \\
\mathrm{N}=19384\end{array}$ \\
\hline Stage I & $22.3 \%$ & $28.1 \%$ & $17.8 \%$ & $27.5 \%$ & $23 \%$ & $27.3 \%$ \\
\hline Stage II & $26.1 \%$ & $28.5 \%$ & $22.1 \%$ & $26 \%$ & $16.7 \%$ & $23.4 \%$ \\
\hline Stage III & $32.3 \%$ & $26.2 \%$ & $34.9 \%$ & $28.5 \%$ & $34.1 \%$ & $28 \%$ \\
\hline Stage IV & $19.3 \%$ & $17.2 \%$ & $25.2 \%$ & $18 \%$ & $26.3 \%$ & $21.3 \%$ \\
\hline
\end{tabular}

Table 3.3 One, three and five years relative survival rates of rectal cancer patients in the Netherlands aged $\leq 70$ years, according to the period of diagnosis and age group.

\begin{tabular}{lcccccc}
\hline & \multicolumn{2}{c}{$1989-1994$} & \multicolumn{2}{c}{$1995-2000$} & \multicolumn{2}{c}{$2001-2010$} \\
& $\leq 40$ years & $41-70$ years & $\leq 40$ years & $41-70$ years & $\leq 40$ years & $41-70$ years \\
& $\mathrm{N}=269$ & $\mathrm{~N}=7630$ & $\mathrm{~N}=281$ & $\mathrm{~N}=8940$ & $\mathrm{~N}=552$ & $\mathrm{~N}=19384$ \\
\hline One years & $83 \%$ & $84 \%$ & $86 \%$ & $86 \%$ & $88 \%$ & $89 \%$ \\
Three years & $62 \%$ & $64 \%$ & $64 \%$ & $68 \%$ & $71 \%$ & $74 \%$ \\
Five years & $52 \%$ & $54 \%$ & $56 \%$ & $58 \%$ & $62 \%$ & $65 \%$ \\
\hline
\end{tabular}

\section{Survival}

When relative survival was compared according to stage, it was found that younger patients presented a slightly better survival per stage for stage I to III rectal cancer compared with middle-aged patients but showed slightly unfavourable survival for stage IV disease (Figure 3.1). In the multivariate analysis of the RER of death for patients with stage I-III rectal cancer, young age was a prognostic factor for better survival (RER $0.82 \mathrm{Cl}$ 0.71-0.94) (Table 3.4). The use of neo-adjuvant radio- or chemoradiation therapy, surgery and adjuvant chemotherapy was associated with increased survival. Male sex, an advanced T-stage, pathological lymph nodes (N1-N2), a signet cell or mucinous morphology, and a poorly undifferentiated grade of differentiation were associated with poor survival. In the multivariate RER analysis, the period of diagnosis was also a significant factor for survival. Patients treated in the periods 1989-1994 (RER $1.67 \mathrm{Cl}$ 1.54-1.80) and 1995-2000 (RER 1.43 Cl 1.33-1.53) exhibited lower survival scores compared with patients treated between 2001 and 2010. 


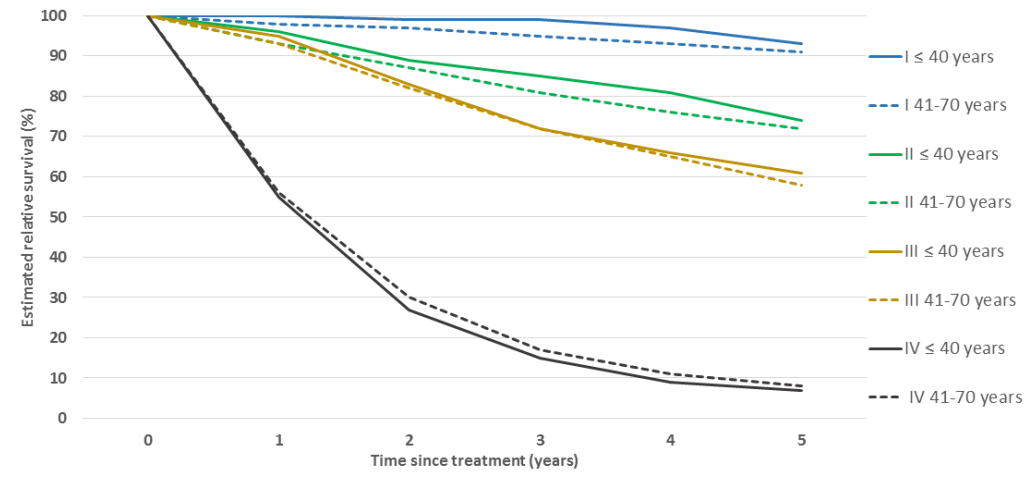

Figure 3.1 The estimated relative survival of all 37056 rectal cancer patients in the Netherlands between 1989-2010 according to stage and stratified by age ( $\leq 40$ years and $\mathbf{4 1 - 7 0}$ years). The estimated 5 -years relative survival rates for patients $\leq 40$ years were $93 \%, 74 \%, 61 \%$ and $7 \%$ for stage I-IV respectively. For patients $41-70$ years the estimated 5 -years relative survival rates were $91 \%$, $72 \%, 58 \%$ and $8 \%$ respectively.

In the multivariate analysis of the RER of death in patients with metastatic disease by presentation (stage IV), young age was not a prognostic factor (RER $1.04 \mathrm{Cl}$ 0.91-1.18) (data not shown). Male sex (RER $0.93 \mathrm{Cl}$ 0.89-0.99) was associated with improved survival. Mucinous and signet cell carcinomas and a poorly undifferentiated grade of differentiation were prognostic factors for poor survival. Patients diagnosed in the periods 1989-1994 (RER $1.33 \mathrm{Cl} 1.24-1.43$ ) and 1995-2000 (RER 1.23 Cl 1.15-1.31) exhibited lower survival rates compared with patients treated between 2001 and 2010.

\section{Adjuvant chemotherapy}

In stage I-III rectal cancer patients, adjuvant chemotherapy was associated with better survival (RER $0.76 \mathrm{Cl} 0.70-0.83$ ). In the univariate analysis performed in patients aged $\leq 40$ years, the use of adjuvant chemotherapy was associated with improved survival for patients with pN1 (RER $0.28 \mathrm{Cl} 0.15-0.53$ ) or stage III rectal cancer (RER $0.55 \mathrm{Cl}$ $0.38-0 ., 78)$. Young patients treated with adjuvant chemotherapy in the period 19891994 had an increased risk of death (RER $2.47 \mathrm{Cl} 1.40-4.34$ ) compared with patients treated during the same period who did not receive adjuvant chemotherapy. For all other examined variables, no significant correlations were found. 
Table 3.4 Uni- and multivariate relative excess risk of death in stage I-III rectal cancer patients in the Netherlands between 1989-2010.

\begin{tabular}{|c|c|c|c|c|c|}
\hline & & & variate & & tivariate \\
\hline & $\mathrm{N}=29734(\%)$ & RER & $95 \% \mathrm{Cl}$ & RER & $95 \% \mathrm{Cl}$ \\
\hline Male & $18058(60.7)$ & 1.1 & $1.04-1.16$ & 1.08 & $1.02-1.14$ \\
\hline Female & $11676(39.3)$ & 1.00 & & 1.00 & \\
\hline Young & $834(2.8)$ & 0.99 & $0.86-1.14$ & 0.82 & $0.71-0.94$ \\
\hline Middle-aged & $28900(97.2)$ & 1.00 & & 1.00 & \\
\hline pT stage & & & & & \\
\hline $\mathrm{T} 1$ & 3431 (11.5) & 1,00 & & 1,00 & \\
\hline $\mathrm{T} 2$ & $8698(29.2)$ & 1.25 & $1.06-1.48$ & 1.35 & $1.14-1.59$ \\
\hline T3 & $14790(49.7)$ & 3.35 & $2.89-3.88$ & 3,00 & $2.58-3.49$ \\
\hline T4 & $1326(4.5)$ & 7.32 & $6.29-8.51$ & 4.86 & $4.14-5.7$ \\
\hline Tx & $1462(4.9)$ & 3.14 & $2.22-4.43$ & 2.41 & $1.72-3.38$ \\
\hline pN stage & & & & & \\
\hline NO & $14002(47.1)$ & 1,00 & & 1,00 & \\
\hline N1 & $6781(22.8)$ & 2.07 & $1.94-2.2$ & 1.75 & $1.64-1.87$ \\
\hline N2 & $3126(10.5)$ & 3.35 & $3.1-3.63$ & 2.61 & $2.4-2.83$ \\
\hline $\mathrm{Nx}$ & 5825 (19.6) & 1.64 & $1.51-1.51$ & 1.29 & $1.18-1.41$ \\
\hline Neo adjuvant treatment & & & & & \\
\hline No radiotherapy & $19357(65.1)$ & 1,00 & & 1,00 & \\
\hline Radiotherapy & $10337(34.9)$ & 0.64 & $0.6-0.68$ & 0.78 & $0.72-0.84$ \\
\hline No radiochemotherapy & $28242(95.0)$ & 1,00 & & 1,00 & \\
\hline Radiochemotherapy & $1492(5.0)$ & 0.81 & $0.7-0.94$ & 0.78 & $0.67-0.91$ \\
\hline No surgery & $854(2.9)$ & 1,00 & & 1,00 & \\
\hline Surgery & $28880(97.1)$ & 0.09 & $0.08-0.1$ & 0.19 & $0.17-0.21$ \\
\hline No adjuvant chemotherapy & $25954(87.3)$ & 1,00 & & 1,00 & \\
\hline Adjuvant chemotherapy & $3780(12.7)$ & 1.07 & $1,00-1.16$ & 0.76 & $0.7-0.83$ \\
\hline No adjuvant radiotherapy & $25815(86.8)$ & 1,00 & & 1,00 & \\
\hline Adjuvant radiotherapy & 3919 (13.2) & 1.92 & $1.8-2.05$ & 1.16 & $1.07-1.25$ \\
\hline Morphology & & & & & \\
\hline Adenocarcinoma & $26889(90.4)$ & $1 \cdot 00$ & & $1 \cdot 00$ & \\
\hline Mucinous carcinoma & $2690(9.1)$ & 1.45 & $1.34-1.57$ & 1.18 & $1.09-1.28$ \\
\hline Signet cell carcinma & $155(0.5)$ & 5.24 & 4.29-6.39 & 2.36 & $1.92-2.9$ \\
\hline Histopathological grade of different & & & & & \\
\hline Well differentiated (G1) & $2144(7.2)$ & 0.88 & $0.78-0.99$ & 0.94 & 0.84-1.05 \\
\hline Moderately differentiated (G2) & $17569(59.1)$ & $1 \cdot 00$ & & $1 \cdot 00$ & \\
\hline Poorly -undifferentiated (G3-G4) & 3341 (11.2) & 2.1 & $1.96-2.24$ & 1.66 & $1.55-1.78$ \\
\hline Missing & $6680(22.5)$ & 1,00 & $0.93-1.07$ & 1.03 & $0.95-1.11$ \\
\hline Period of diagnosis & & & & & \\
\hline $1989-1994$ & 6525 (21.9) & 1.93 & $1.81-2.05$ & 1.67 & $1.54-1.8$ \\
\hline $1995-2000$ & $7538(25.4)$ & 1.57 & $1.47-1.67$ & 1.42 & $1.33-1.53$ \\
\hline $2001-2010$ & $15671(52.7)$ & 1.00 & & 1.00 & \\
\hline
\end{tabular}

\section{Discussion}

Young rectal cancer patients are more likely to have advanced-stage disease, and their tumours exhibit unfavourable characteristics. Nevertheless, their survival rates are equal to those of middle-aged patients. 
In the present study, young rectal cancer patients had more advanced stage of disease compared to middle-aged patients. This pattern has also been observed in other studies, in which younger patients were found to present with more advanced disease. ${ }^{6,9,10,21}$ As in the present study, the patients in these studies had unfavourable tumour morphology. In both age groups, stage migration was observed during the study period, resulting in an increase in patients with stage IV rectal cancer and a decrease in patients with stage II rectal cancer. This phenomenon has been reported previously and is probably due to the use of improved diagnostic and staging modalities, such as CT, PET-CT and MRI. ${ }^{22}$

Survival has improved since the introduction and implementation of TME surgery and neo-adjuvant treatment. ${ }^{23}$ Also in the present study this improvement was found. When survival was analysed according to stage, we found similar survival rates for young and middle-aged rectal cancer patients. In a study by $\mathrm{O}^{\prime}$ Connel et al., ${ }^{9}$ slightly unfavourable relative survival rates were reported compared with our study, with fiveyear relative survival rates of $88 \%, 75 \%$ and $51 \%$ being observed for stage I-III rectal cancer, respectively, in young patients. You et al. ${ }^{10}$ reported much higher survival rates of $92-93 \%, 97-98 \%$ and $69-74 \%$ for stage I, II, and III disease, respectively. These remarkably high survival rates are probably due to the single-institution character of this study and selection bias, as patients who did not receive chemotherapy were excluded. Furthermore, patients up to the age of 50 were defined as young patients. Comparing the results of the present study to other studies on young rectal cancer patients is difficult because the studies on this topic have mainly been single-centre experiences with small sample sizes and did not include all clinical and survival information. Furthermore, nearly all of these studies included both colon and rectal cancer patients. More comparable results to our study are observed in studies employing the SEER database. ${ }^{5,9}$ However, these studies do not include information concerning treatment, and stage information is missing in a large proportion of the patients.

Compared with older rectal cancer patients, young patients are more likely to present with unfavourable histology. As in other studies, these tumour characteristics were associated with poor survival. ${ }^{24-26}$ In the present study, $16.6 \%$ of the young patients had poorly differentiated tumours, compared with $24.3 \%$ in an American SEER database study. ${ }^{9}$ This difference could be explained by population differences (e.g., race) between the American and Dutch populations. Furthermore, these differences could be due to the large number of missing values in our study and the fact that interpretation can differ between Dutch and American pathologists. Recent data on the incidence rates of mucinous tumours in young patients are only available from small studies, mainly involving colorectal cancer patients; in these studies, the reported rate of mucinous tumours varied widely, from $6.1 \%-32 \% .^{8,10,21}$ Older studies recorded an 
average rate of mucinous tumours in young colorectal patients of $21 \%{ }^{27,28}$ The differences in reported rates may be due to single-centre experiences, the inclusion of both rectal and colon cancers and the small sample sizes of these studies. We believe that the rates we observed in terms of differentiation grade and tumour morphology reflect the normal distribution in a western population because of the populationbased character of this study.

In patients with local disease (stage I-III), the two patient groups were equally likely to be treated with surgery and neo-adjuvant treatment. However, young patients with stage I-III disease received adjuvant chemotherapy more often, with $22.8 \%$ of the young patients receiving adjuvant chemotherapy, compared with $12.4 \%$ of the middleaged patients. Interestingly, in this study, adjuvant chemotherapy was associated with improved survival (RER $0.76 \mathrm{Cl} 0.70-0.83$ ). In an exploratory analysis, we found that the use of adjuvant chemotherapy in patients with pN1 or stage III disease resulted in improved survival. Adjuvant chemotherapy in patients with pN2 disease was not found to significantly improve survival, but this could also be due to the low patient numbers in this group.

In more recent randomised controlled trials, such as the Proctor-Script, EORTC 22921, CHRONICLE trials, no survival benefit was observed with the use of adjuvant chemotherapy after neo-adjuvant (chemo-)radiotherapy. ${ }^{29-31}$ In the QUASAR trial were only $21 \%$ received radiotherapy, a small positive effect of adjuvant chemotherapy on survival was seen, especially in stage III rectal cancer. ${ }^{32}$ Although all these trials showed no additional survival benefit for adjuvant chemotherapy, our population-based study showed additional survival benefit. This finding is supported by another populationbased study from Sweden, in which a positive association of adjuvant chemotherapy on survival was observed for stage III patients treated with neo-adjuvant radiotherapy. ${ }^{33}$ Patients aged 50-60 years and those with proximal tumours had a statistically significant overall survival benefit. In the most recent meta-analyses a possible positive effect of adjuvant chemotherapy in proximal rectal tumours was also found but they also state that more research is needed. ${ }^{19}$ Furthermore, age was not addressed and only a few patients in the study can be considered young.

One could argue that this population-based study is less reliable because of a significant effect of selection bias. However, we performed an exploratory analysis only in younger patients. We believe that selection bias may play less of a role in younger patients, who generally have fewer co-morbidities and better clinical characteristics. Therefore, the observed effect on survival is interesting, and although our results need to be interpreted with caution due to limitations related to the population-based character of this study, there could be an indication for adjuvant chemotherapy in younger patients with stage III disease. It is possible that the observed differences in tumour morphology 
between the age groups represent different biological behaviour, rendering these tumours more susceptible to adjuvant chemotherapy. Ultimately, our results should stimulate further research about adjuvant chemotherapy in rectal cancer with a focus on patient age and/or biological tumour behaviour, rather than changing the current national guidelines for rectal cancer.

This large population-based study provides information about tumour and treatment characteristics and highlights that young patients present with more aggressive and more advanced tumours. Despite these characteristics, their survival rates are equal to middle-aged patients. After correction for confounding variables including treatment and tumour characteristics, young age was even shown to be a prognostic factor for improved survival. Our results suggest that young patients do not fare worse than older patients and therefore should be equally treated. Studies should focus more on age and biological tumour behaviour and the effect of treatment on these characteristics. Future studies have to be awaited before younger patients with rectal cancer are treated with adjuvant chemotherapy.

The strength of this study is that it presents nationwide, population-based, high-quality data, with available information about disease stage, tumour morphology, treatment and long-term outcomes. To our knowledge, this is the largest population-based study on rectal cancer in young patients including clinical, pathological treatment and survival information.

\section{Conclusion}

Young patients present with more advanced disease and less favourable tumour morphology compared with middle-aged patients. Despite these unfavourable characteristics, the survival rates in these groups are equal, and young age is even a prognostic factor for improved survival. Young patients received adjuvant chemotherapy more often compared with middle-aged patients, and treatment with chemotherapy was associated with improved survival in young patients with pN1 or stage III disease. Nevertheless, these results have to be interpreted with caution, and further research will be needed to assess the effect of adjuvant chemotherapy in young rectal cancer patients. 


\section{References}

1. International Agency for Research on Cancer, Globocan 2012. http://globocan.iarc.fr/Pages/fact_ sheets_cancer.aspx?cancer=colorectal accessed on 12-07-2014. 2014.

2. IKNL Cijfers over kanker. http://www.cijfersoverkanker.nl/p=54845cc3c08f5, accessed 12-7-2014. 2014.

3. Atkin WS, Cuzick J. Screening for colorectal cancer. Lancet 1993;341:1667.

4. Davis DM, Marcet JE, Frattini JC, et al. Is it time to lower the recommended screening age for colorectal cancer? J Am Coll Surg 2011;213:352-61.

5. Meyer JE, Narang T, Schnoll-Sussman FH, et al. Increasing incidence of rectal cancer in patients aged younger than 40 years: an analysis of the surveillance, epidemiology, and end results database. Cancer 2010;116:4354-9.

6. Endreseth $\mathrm{BH}$, Romundstad $\mathrm{P}, \mathrm{Myrvold} \mathrm{HE}$, et al. Rectal cancer in the young patient. Dis Colon Rectum 2006;49:993-1001.

7. Fairley TL, Cardinez CJ, Martin J, et al. Colorectal cancer in U.S. adults younger than 50 years of age, 1998-2001. Cancer 2006;107(5 Suppl):1153-61.

8. Ganapathi S, Kumar D, Katsoulas N, et al. Colorectal cancer in the young: trends, characteristics and outcome. Int J Colorectal Dis 2011;26:927-34.

9. O'Connell JB, Maggard MA, Liu JH, et al. Are survival rates different for young and older patients with rectal cancer? Dis Colon Rectum 2004;47:2064-9.

10. You YN, Dozois EJ, Boardman LA, et al. Young-onset rectal cancer: presentation, pattern of care and long-term oncologic outcomes compared to a matched older-onset cohort. Ann Surg Oncol 2011;18: 2469-76.

11. O'Connell JB, Maggard MA, Livingston EH, Yo CK. Colorectal cancer in the young. Am J Surg 2004;187: 343-8.

12. Smith C, Butler JA. Colorectal cancer in patients younger than 40 years of age. Dis Colon Rectum 1989; 32:843-6.

13. Alici S, Aykan NF, Sakar B, et al. Colorectal cancer in young patients: characteristics and outcome. Tohoku J Exp Med 2003;199:85-93.

14. Lin JT, Wang WS, Yen CC, et al. Outcome of colorectal carcinoma in patients under 40 years of age. $J$ Gastroenterol Hepatol 2005;20:900-5.

15. Wolpin BM, Mayer RJ. Systemic treatment of colorectal cancer. Gastroenterology 2008;134:1296-310.

16. Brenner H, Kloor M, Pox CP. Colorectal cancer. Lancet 2014;383:1490-502.

17. Glimelius B, Tiret E, Cervantes A, Arnold D. Rectal cancer: ESMO Clinical Practice Guidelines for diagnosis, treatment and follow-up. Ann Oncol 2013;24 Suppl 6:vi81-8.

18. van de Velde CJ, Boelens PG, Borras JM, et al. EURECCA colorectal: multidisciplinary management: European consensus conference colon \& rectum. Eur J Cancer 2014;50:1.

19. Breugom AJ, Swets M, Bosset JF, et al. Adjuvant chemotherapy after preoperative (chemo)radiotherapy and surgery for patients with rectal cancer: a systematic review and meta-analysis of individual patient data. Lancet Oncol 2015;16:200-7.

20. Ederer F, Axtell LM, Cutler SJ. The relative survival rate: a statistical methodology. Nat/ Cancer Inst Monogr 1961;6:101-21.

21. Liang JT, Huang KC, Cheng AL, et al. Clinicopathological and molecular biological features of colorectal cancer in patients less than 40 years of age. Br J Surg 2003;90:205-14.

22. Lemmens VE, de Haan N, Rutten $\mathrm{HJ}$, et al. Improvements in population-based survival of patients presenting with metastatic rectal cancer in the south of the Netherlands, 1992-2008. Clin Exp Metastasis 2011;28:283-90.

23. den Dulk M, Krijnen $P$, Marijnen CA, et al. Improved overall survival for patients with rectal cancer since 1990: the effects of TME surgery and pre-operative radiotherapy. Eur J Cancer 2008;44:1710-6.

24. Hyngstrom JR, Hu CY, Xing $\mathrm{Y}$, et al. Clinicopathology and outcomes for mucinous and signet ring colorectal adenocarcinoma: analysis from the National Cancer Data Base. Ann Surg Oncol 2012;19: 2814-21.

25. Hugen N, Verhoeven RH, Radema SA, et al. Prognosis and value of adjuvant chemotherapy in stage III mucinous colorectal carcinoma. Ann Oncol 2013;24:2819-24. 
26. Hugen $\mathrm{N}$, Verhoeven $\mathrm{RH}$, Lemmens $\mathrm{VE}$, et al. Colorectal signet-ring cell carcinoma: benefit from adjuvant chemotherapy but a poor prognostic factor. Int J Cancer 2015;136:333-9.

27. Griffin PM, Liff JM, Greenberg RS, Clark WS. Adenocarcinomas of the colon and rectum in persons under 40 years old. A population-based study. Gastroenterology 1991;100:1033-40.

28. Lee PY, Fletcher WS, Sullivan ES, Vetto JT. Colorectal cancer in young patients: characteristics and outcome. Am Surg 1994;60:607-12.

29. Bosset JF, Calais G, Mineur L, et al. Fluorouracil-based adjuvant chemotherapy after preoperative chemoradiotherapy in rectal cancer: long-term results of the EORTC 22921 randomised study. Lancet Oncol 2014;15:184-90.

30. Glynne-Jones R, Counsell N, Quirke P, et al. Chronicle: results of a randomised phase III trial in locally advanced rectal cancer after neoadjuvant chemoradiation randomising postoperative adjuvant capecitabine plus oxaliplatin (XELOX) versus control. Ann Oncol 2014;25:1356-62.

31. Breugom AJ, van GW, Muller EW, et al. Adjuvant chemotherapy for rectal cancer patients treated with preoperative (chemo)radiotherapy and total mesorectal excision: a Dutch Colorectal Cancer Group (DCCG) randomized phase III trial. Ann Oncol 2015;26:696-701.

32. Gray R, Barnwell J, McConkey C, et al. Adjuvant chemotherapy versus observation in patients with colorectal cancer: a randomised study. Lancet 2007;370:2020-9.

33. Tiselius C, Gunnarsson U, Smedh K, et al. Patients with rectal cancer receiving adjuvant chemotherapy have an increased survival: a population-based longitudinal study. Ann Oncol 2013;24:160-5. 
CHAPTER 4 Are there age related differences in morbidity and mortality after surgery for locally advanced and locally recurrent rectal cancer?

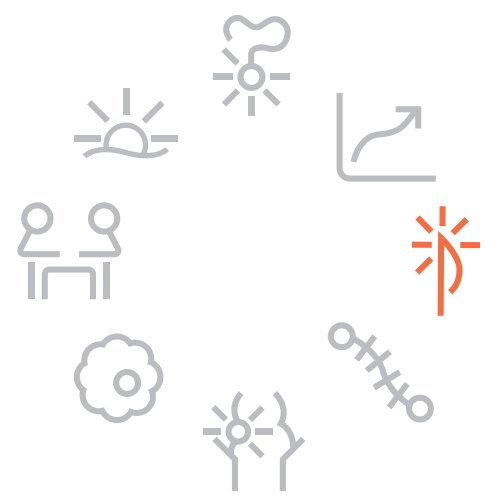

R.G. Orsini

S.J. Bosman

T.A. Vermeer

G.A. Simkens

G.A. Nieuwenhuijzen

I.H. de Hingh

H.J. Rutten 


\section{Abstract}

\section{Introduction}

The aim of this study is to describe the differences in morbidity and mortality of elderly CT4 (CT4RC) and locally recurrent rectal cancer (LRRC) patients who are treated with curative intent.

\section{Methods}

Patients receiving curative surgery for primary cT4RC or LRRC between 2005 and 2013 in the Catharina Hospital (Eindhoven, the Netherlands) were selected retrospectively. Analyses on treatment, morbidity, survival and risk factors for one-year mortality were done separately for both CT4RC and LRRC and stratified by age $(<75$ years and $\geq 75$ years).

\section{Results}

473 (362 cT4RC and 161 LRRC) patients were selected including 49 (16\%) cT4RC and 28 (17\%) LRRC patients $\geq 75$ years. Grade III/IV complications occurred in $18 \%$ and $50 \%$ of the elderly CT4RC and LRRC patients compared to $19 \%$ and $32 \%$ in younger CT4RC and LRRC patients. Mortality increased from $8 \%$ (1-month) to $20 \%$ (1-year) in elderly cT4RC compared to $1,5 \%$ and $5 \%$ in cT4RC < 75 years. In LRRC 1 -year mortality was $19 \%$ for young and $25 \%$ for elderly patients. Age $\geq 75$ years (OR 3.83 ) and an exenteration (OR 6.77) were predictive for one-year mortality in cT4RC and a grade IV complication in both cT4 (OR 13.1) and LRRC (OR 5.66).

\section{Conclusion}

Morbidity after surgery for CT4RC and LRRC is high but regardless of age in cT4 rectal cancer patients. One-month mortality in elderly cT4RC patients is a high underestimation of the risk of dying in the first post-operative year. 


\section{Introduction}

The treatment for patients with locally advanced rectal cancer (LARC) and locally recurrent rectal cancer (LRRC) is challenging. Curative treatment consists of neoadjuvant treatment followed by major surgery and in most of these cases an extraanatomical and multivisceral resection is required. These intensive treatment regimens are needed in order to provide the best chance for long-term survival. ${ }^{1}$ However, perioperative morbidity is high with a reported median complication rate of $41.5 \%-57 \%{ }^{2,3}$

Elderly patients have difficulties to cope with complications, their recovery process takes longer and mortality is increased during the first post-operative year. ${ }^{4-6}$ As elderly often have multiple comorbidities and their physical condition ranges from fit to frail, treatment for advanced and recurrent cases treatment is even more challenging. Previous studies reported an increased in elderly patients for being not curatively treated, this affects their survival negatively. ${ }^{7,8}$ In a Dutch study only $62 \%$ of the elderly patients ( $\geq 75$ years) compared to $87 \%$ of the patients aged $<75$ years with a stage I-III rectal cancer were treated according to the guidelines. ${ }^{6}$ Age was the primary reason for withholding curative treatment in this study. However, the patients' chronical age is a poor reflection of their actual performance status. ${ }^{9,10}$

Little is known about how elderly with LARC or LRRC endure intensive treatment regimens with neo-adjuvant therapy followed by major rectal surgery and multi-visceral resections. The primary aim of this study is to describe the morbidity and mortality of CT4 rectal cancer (cT4RC) and LRRC in elderly patients ( $\geq 75$ years) who are treated with curative intent. These results are compared to younger patients in order to investigate differences between these groups. The secondary aim of this study was to identify risk factors and the effect of age on one-year mortality for both treatment groups.

\section{Materials and methods}

\section{Patients and treatment}

The Catharina Hospital (Eindhoven, the Netherlands) is a tertiary referral center for patients with LARC or LRRC. For this study, patients receiving curative surgery for primary cT4 or locally recurrent rectal cancer between 2005 and 2013 were selected. All patients with CT4RC had a histologically proven rectal cancer. In addition, all patients with CT4RC or LRRC received preoperative magnetic resonance imaging (MRI) of the pelvis for accurate staging before and after any preoperative treatment. A pretreatment computerized tomography (CT) of the chest and abdomen was 
performed in order to exclude the presence of distant metastases. A positron emission tomography (PET-CT) was used to distinguish between fibroses or LRRC when a histological biopsy could not be obtained. Patients with cT4RC received neo-adjuvant treatment according to the Dutch National Guidelines for rectal cancer. ${ }^{11}$ The majority of patients with CT4RC received long course chemoradiation up to $50.4 \mathrm{~Gy}$ in 28 fractions. Different neo-adjuvant treatment schemes of the chemotherapy component were used during the study period: 5-FU and leucovorin (5FU-Bolus), a combination of capecitabine and oxaliplatin (CORE), avastin and capectitabine (RAX) and capecitabine only, which has become the standard. Details of these treatment regimens and influence on outcome have been reported earlier. ${ }^{12}$ LRRC patients who were previously irradiated, received re-irradiation with a dose of $30 \mathrm{~Gy}(5 \times 1.6 \mathrm{~Gy}$ a week), with concomitant chemotherapy. LRRC patients, who did not receive irradiation in the past, received full course irradiation with chemotherapy. ${ }^{13}$

Prior to surgery all patients received a second MRI and CT of the chest and abdomen to evaluate the occurrence of downstaging and the presence of distant metastasis. Surgery was scheduled 8-12 weeks after completion of neo-adjuvant treatment. In both groups, patients underwent a low anterior resection (LAR) or an abdominoperineal excision (APE) according to TME principles. In some cases, an abdominosacral resection (ASR), multivisceral resection or total debulking was required in order to achieve a radical resection. An APE or total exenteration was performed in a supine position, allowing simultaneous access from both the abdominal and perineal side. Details of this surgical procedure have been described elsewhere. ${ }^{14,15}$ If primary closure of the vagina or perineal wound was impossible, a vertical rectus abdominis myocutaneous (VRAM) flap with or without skin-island was used. Intra-operative radiotherapy boost (IORT) of 10-15 Gy was applied when a positive resection margin was suspected or confirmed by intra-operative frozen section. Details on this multimodality treatment strategy and the influence on outcome have been described before. $^{16,17}$ Patients who were not treated with curative intent or who had distant metastasis at the time of presentation were excluded from this study.

\section{Clinical data and follow-up}

Data on treatment and pathology are recorded prospectively but some additional clinical (e.g. complications, hospital admission) and demographic data were retrospectively extracted from medical records. Complications were scored using the Clavien-Dindo classification of surgical complications. ${ }^{18}$ Follow-up data was extracted from medical records or by contacting the patient, the referral hospital or patients' general practitioner by telephone. Follow-up was calculated as the time from the day of surgery to death or the date of last contact. The minimum follow-up of all patients was at least one year (if alive). If a patient had died during follow-up the specific cause of death was scored. 


\section{Statistical analyses}

Statistical analyses were performed using the SPSS Statistics 22.0 software (SPSS Inc., Chicago, IL, USA). All analyses were separately done for both CT4RC and LRRC. Comparisons within these groups were based on age ( $<75$ years and $\geq 75$ years). No comparisons were made between the two treatment groups. Intergroup comparisons were analyzed using chi-square tests or independent $t$-tests when appropriate. A $p$ value of $\leq 0.05$ was considered statistically significant. Survival rates for both treatment groups were estimated separately and stratified by age group using the Kaplan-Meijer method. To analyze risk factors for mortality, Binary Regression analyses were performed for both treatment groups with a cut-off point of one-year (patients who died within the first post-operative year). For this analyses all complications were grouped according to the Clavien Dindo classification. Covariates with trend-significant effect $(p<.10)$ were selected for multivariable binary regression analysis.

\section{Results}

A total of 473 patients were selected. Of the 362 cT4RC patients, $49(16 \%)$, patients were $\geq 75$ years. Of the 161 LRRC patients, 28 (17\%) were $\geq 75$ years. Median follow-up was 4.3 years for CT4RC patients and 2.4 years for LRRC patients. Other clinical and demographic characteristics for CT4RC patients are presented in Table 4.1. Elderly patients were less often treated with chemoradiation and more often treated with short or long course radiotherapy compared to younger patients $(p<0.001)$. An APR or Hartmann's procedure was performed more often than a low anterior resection in the elderly compared to younger patients. In $67 \%$ of the elderly patients and $54 \%$ of the younger patients a multivisceral resection was performed $(p=0.09)$. The use of IORT was similar in both groups. A radical resection was less often achieved in elderly compared to younger patients $(p=0.008)$.

Clinical and demographic characteristics of LRRC patients are presented in Table 4.2. In LRRC patients, there were no differences in comorbidities and ASA classifications. Elderly LRRC patients were more often treated with full-course irradiation with or without concomitant chemotherapy compared to younger patients. Younger patients were often re-irradiated with or without concomitant chemotherapy compared to older patients $(p=0.048)$. No differences in the type of surgery or multivisceral resections were observed between both groups. IORT was more often used in younger LRRC patients compared to older patients $(p=0.037)$. 
Table 4.1 Demographic, clinical and tumor characteristics of cT4 rectal cancer patients stratified by age.

\begin{tabular}{|c|c|c|c|}
\hline & $\begin{array}{c}<75 \text { years } \\
n=263 \\
n(\%)\end{array}$ & $\begin{array}{c}\geq 75 \text { years } \\
n=49 \\
n(\%)\end{array}$ & p-value \\
\hline Median age in years at time of surgery (range) & $62.8(34.4-74.7)$ & $77.8(75-87.8)$ & $<0.001$ \\
\hline Median follow-up in years $( \pm S D)$ & $4.4(2.5)$ & $2.7(2.5)$ & 0.002 \\
\hline Male & $152(57.8)$ & $28(57.1)$ & 0.90 \\
\hline \multicolumn{4}{|l|}{ Comorbidity } \\
\hline None & $93(35.4)$ & $8(16.3)$ & \\
\hline 1 comorbidity & $81(30.8)$ & $13(26.5)$ & \\
\hline 2 comorbidities & $41(15.6)$ & $9(18.4)$ & \\
\hline$\geq 3$ comorbidities & $32(12.2)$ & $15(30.6)$ & \\
\hline Missing & $16(6.1)$ & $4(8.2)$ & 0.004 \\
\hline \multicolumn{4}{|l|}{ ASA classification } \\
\hline$|-I|$ & $209(79.5)$ & $34(69.4)$ & \\
\hline III & $40(15.2)$ & $14(28.6)$ & \\
\hline Missing & $14(5.3)$ & $1(2.0)$ & 0.057 \\
\hline \multicolumn{4}{|l|}{ Neo-adjuvant treatment } \\
\hline None & - & $2(4.1)$ & \\
\hline Short course radiotherapy (5x5Gy) & $9(3.4)$ & $5(10.2)$ & \\
\hline Long course radiotherapy & $9(3.4)$ & $8(16.3)$ & \\
\hline Chemoradiation & $243(92.4)$ & $34(69.4)$ & \\
\hline Missing & $2(0.8)$ & - & $<0.001$ \\
\hline \multicolumn{4}{|l|}{ Type of surgery } \\
\hline Low anterior resection & $112(42.6)$ & $17(34.7)$ & \\
\hline Abdominoperineal resection & $116(44.1)$ & $25(51.0)$ & \\
\hline Abdominosacral resection & $9(3.4)$ & - & \\
\hline Exenteration & $21(8.0)$ & $2(4.1)$ & \\
\hline Hartmann resection & $5(1.9)$ & $5(10.2)$ & 0.014 \\
\hline Extended (multivisceral) resection & $143(54.4)$ & $33(67.3)$ & 0.09 \\
\hline Intra operative radiotherapy (IORT) & $175(66.5)$ & $30(61.2)$ & 0.50 \\
\hline Radical resection (R0) & $245(93.2)$ & $40(81.6)$ & 0.008 \\
\hline Median admission time in days $( \pm S D)$ & $9(15)$ & $10(8)$ & 0.16 \\
\hline Median admission time on ICU in days ( \pm SD) & $1(5)$ & $1(4.5)$ & 0.068 \\
\hline
\end{tabular}

\section{Morbidity}

A surgical or radiological intervention due to a complication (grade III) was needed in $12 \%$ of the elderly and $15 \%$ of the younger cT4RC patients ( $p=n s)$. Three (6.1\%) elderly patients developed a life threatening complication (grade IV) compared to 11 patients (4.2\%) younger cT4RC patients $(p=n s)$. Three $(6.1 \%)$ elderly cT4RC died due to complications in the post-operative period compared to four patients $(1.5 \%)$ younger cT4RC patients $(p=0.046)$.

In LRRC, grade III complications occurred in 24 (43\%) elderly patients compared to 38 (29\%) in the younger patients. Two (7.1\%) elderly LRRC patients had a grade IV complication and two (7.1\%) died postoperatively. In the younger age group 5 (3.8\%) patients had a grade IV complication and 3 (2.3\%) died during the post-operative 
period. A more detailed description of the complications in both treatment groups is presented in Table 4.3.

Table 4.2 Demographic, clinical and tumor characteristics of locally recurrent rectal cancer patients stratified by age.

\begin{tabular}{|c|c|c|c|}
\hline & $\begin{array}{c}<75 \text { years } \\
n=133 \\
n(\%)\end{array}$ & $\begin{array}{c}\geq 75 \text { years } \\
n=28 \\
n(\%)\end{array}$ & p-value \\
\hline Median age in years at time of surgery (range) & $63.4(30.7-74.9)$ & $77.7(75.2-86.8)$ & $<0.001$ \\
\hline Median follow-up in years $( \pm S D)$ & $2.3(2.0)$ & $2.6(1.8)$ & 0.51 \\
\hline Male & $88(66.2)$ & $17(60.7)$ & 0.58 \\
\hline \multicolumn{4}{|l|}{ Comorbidity } \\
\hline 1 comorbidity & $53(39.8)$ & $6(21.4)$ & \\
\hline 2 comorbidities & $33(24.8)$ & $9(32.1)$ & \\
\hline$\geq 3$ comorbidities & $47(35.3)$ & $9(46.4)$ & 0.18 \\
\hline \multicolumn{4}{|l|}{ ASA classification } \\
\hline$|-I|$ & $111(83.5)$ & $21(75.0)$ & \\
\hline III & $13(9.8)$ & 5 (17.9) & \\
\hline IV & $1(0.7)$ & - & \\
\hline Missing & $8(6.0)$ & $2(7.1)$ & 0.60 \\
\hline \multicolumn{4}{|l|}{ Neo-adjuvant treatment } \\
\hline None & $5(3.8)$ & - & \\
\hline Re-irradiation only & $7(5.3)$ & $1(3.6)$ & \\
\hline Re-irradiation with concomitant chemotherapy & $77(57.9)$ & $13(46.4)$ & \\
\hline Full course irradiation with concomitant chemotherapy & $41(30.8)$ & $10(35.7)$ & \\
\hline Full course irradiation only & $3(2.3)$ & $4(14.3)$ & 0.048 \\
\hline \multicolumn{4}{|l|}{ Type of surgery } \\
\hline Low anterior resection & $22(16.5)$ & $3(10.7)$ & \\
\hline Abdominoperineal resection & $25(18.8)$ & 5 (17.9) & \\
\hline Hartmann resection & $8(6.0)$ & $2(7.1)$ & \\
\hline Abdominosacral resection & $31(23.3)$ & $4(14.3)$ & \\
\hline Exenteration & $24(18.0)$ & $8(28.6)$ & \\
\hline Debulking & $21(15.8)$ & $4(14.3)$ & \\
\hline Other & $2(1.5)$ & $2(17.1)$ & 0.46 \\
\hline Extended (multivisceral) resection & $82(61.7)$ & $17(60.7)$ & 0.93 \\
\hline Intra operative radiotherapy (IORT) & $125(94.0)$ & $23(82.1)$ & 0.037 \\
\hline Radical resection (R0) & $77(57.9)$ & $18(64.3)$ & 0.34 \\
\hline Median admission time in days $( \pm S D$ ) & $14(8.0)$ & $13(22.0)$ & 0.59 \\
\hline Median admission time on ICU in days ( \pm SD) & $1(3.2)$ & $1.0(2.4)$ & 0.62 \\
\hline
\end{tabular}

\section{Survival and prognostic factors}

Overall and cancer specific survival rates at various timing intervals are presented in Table 4.4. In elderly cT4RC mortality increased from $8 \%$ after one month to $20 \%$ after one year. In younger patients, mortality after one year was 5\%. (Figure 4.1) For LRRC there were no differences between both age groups in mortality rates for various time points. (Figure 4.1) 
Table 4.3 Details on post-operative complications of cT4 and LR rectal cancer patients stratified by age.

\begin{tabular}{|c|c|c|c|c|}
\hline & \multicolumn{2}{|c|}{ cT4 } & \multicolumn{2}{|c|}{ LRRC } \\
\hline & $\begin{array}{c}<75 \text { years } \\
n=263 \\
n(\%)\end{array}$ & $\begin{array}{c}\geq 75 \text { years } \\
n=49 \\
n(\%)\end{array}$ & $\begin{array}{c}<75 \text { years } \\
n=133 \\
n(\%)\end{array}$ & $\begin{array}{c}\geq 75 \text { years } \\
n=28 \\
n(\%)\end{array}$ \\
\hline \multicolumn{5}{|l|}{ Anastomotic leakage } \\
\hline Requiring intervention & $3(1.1)$ & $1(2.0)$ & $3(2.3)$ & $1(3.6)$ \\
\hline Conservative treatment & $5(1.9)$ & - & $1(0.8)$ & - \\
\hline \multicolumn{5}{|l|}{ Presacral Abcess } \\
\hline Requiring intervention & $20(7.6)$ & - & $17(34.7)$ & $5(38.5)$ \\
\hline Conservative treatment & $9(3.4)$ & - & $5(10.2)$ & $1(7.7)$ \\
\hline \multicolumn{5}{|l|}{ Intra Abdominal abcess } \\
\hline Requiring intervention & $7(2.7)$ & - & $14(10.5)$ & $4(14.3)$ \\
\hline Conservative treatment & $3(1.1)$ & $1(2.0)$ & $6(4.5)$ & $1(3.6)$ \\
\hline \multicolumn{5}{|l|}{ Wound infection } \\
\hline Abdominal & $14(5.3)$ & $2(4.1)$ & $15(12.0)$ & $1(3.8)$ \\
\hline Perineal & $9(3.4)$ & $4(8.2)$ & $17(12.8)$ & $3(10.7)$ \\
\hline Platzbauch & $7(2.9)$ & $2(4.1)$ & $3(2.3)$ & $3(10.7)$ \\
\hline Ileus & $23(8.7)$ & $8(16.3)$ & $19(14.3)$ & $3(10.7)$ \\
\hline Other requiring relaparotomy & $19(7.2)$ & $5(10.2)$ & $13(9.8)$ & $2(7.1)$ \\
\hline Urologic & $44(16.7)$ & $10(20.4)$ & $38(28.6)$ & $7(25.0)$ \\
\hline Pulmonary & $19(7.2)^{a}$ & $9(18.4)^{\mathrm{a}}$ & $18(13.5)$ & $7(25.0)$ \\
\hline Cardiac & $9(3.4)$ & $4(8.2)$ & 1 & - \\
\hline CVA & $4(1.5)$ & $2(4.1)$ & $2(1.6)$ & - \\
\hline \multicolumn{5}{|c|}{ Complication Grade according to Clavien Dindo ${ }^{18}$} \\
\hline None & $115(43.7)$ & $15(30.6)$ & $33(24.8)$ & $1(3.6)$ \\
\hline Grade I-II & $89(33.8)$ & $22(44.9)$ & 51 (38.3) & $9(32.1)$ \\
\hline Grade IIIa+IIIb & $40(15.2)$ & $6(12.2)$ & $38(28.6)$ & $24(42.9)$ \\
\hline Grade IV & $11(4.2)$ & $3(6.1)$ & $5(3.8)$ & $2(7.1)$ \\
\hline Grade V & - $\quad 4(1.5)^{\mathrm{b}}$ & - $\quad 3(6.1)^{b}$ & $3(2.3)$ & $2(7.1)$ \\
\hline Missing & $4(1.5)$ & - & $3(2.3)$ & $2(7.1)$ \\
\hline
\end{tabular}

${ }^{a} p=0.012,{ }^{b} p=0.046$.

In multivariable analysis of cT4Rc, age $\geq 75$ years was a predictive factor (OR $3.83 \mathrm{Cl}$ 1.19-12.6 $\mathrm{p}=0.027$ ) for one year mortality. A total exenteration (OR $6.77 \mathrm{Cl} 1.13-40-4$ $\mathrm{p}=0.036$ ) and a grade IV complication (OR $13.12 .74-63.2 \mathrm{p}=0.001$ ) were both strong predictive factors for one-year mortality. (see Table 4.5) In LRRC grade IV complication was the only predictive factor (OR $5.66 \mathrm{Cl} 1.11-28.7 \mathrm{p}=0.036$ ) for one year mortality. All other factors showed no significant effect, probably due to low numbers and a multivariable analyses was therefore considered not relevant. 
Table 4.4 Overall, cancer specific and relapse free survival rates for cT4 and LR rectal cancer patients stratified by age ( $<75$ and $\geq 75$ years).

\begin{tabular}{|c|c|c|c|c|}
\hline \multicolumn{5}{|l|}{ Overall survival } \\
\hline & cT4 $<75 y$ ys & cT4 $\geq 75$ yrs & LR $<75$ yrs & $L R \geq 75$ yrs \\
\hline 1-month & 0.99 & 0.92 & 0.97 & 0.93 \\
\hline 6-months & 0.98 & 0.88 & 0.91 & 0.86 \\
\hline 1-year & 0.95 & 0.8 & 0.81 & 0.75 \\
\hline 3-years & 0.82 & .0 .56 & 0.52 & 0.48 \\
\hline \multirow[t]{2}{*}{ 5-years } & 0.7 & 0.42 & 0.32 & 0.27 \\
\hline & \multicolumn{2}{|c|}{$\mathrm{p}<0.001$} & \multicolumn{2}{|c|}{ NS } \\
\hline \multicolumn{5}{|c|}{ Cancer specific survival } \\
\hline & cT4 $<75 y$ ys & cT4 $\geq 75$ yrs & LR $<75$ yrs & $L R \geq 75$ yrs \\
\hline 1-year & 0.97 & 0.88 & 0.89 & 0.84 \\
\hline 3-years & 0.87 & 0.69 & 0.58 & 0.61 \\
\hline \multirow[t]{2}{*}{ 5-years } & 0.79 & 0.65 & 0.35 & 0.35 \\
\hline & \multicolumn{2}{|c|}{$p=0.013$} & \multicolumn{2}{|c|}{ NS } \\
\hline \multicolumn{5}{|c|}{ Disease free survival } \\
\hline & cT4 < 75 yrs & cT4 $\geq 75$ yrs & LR $<75$ yrs & $\mathrm{LR} \geq 75$ yrs \\
\hline 1-year & 0.87 & 0.89 & 0.67 & 0.63 \\
\hline 3-years & 0.76 & 0.61 & 0.33 & 0.37 \\
\hline \multirow[t]{2}{*}{ 5-years } & 0.69 & 0.61 & 0.28 & 0.32 \\
\hline & \multicolumn{2}{|c|}{ NS } & \multicolumn{2}{|c|}{ NS } \\
\hline
\end{tabular}

NS: not significant.
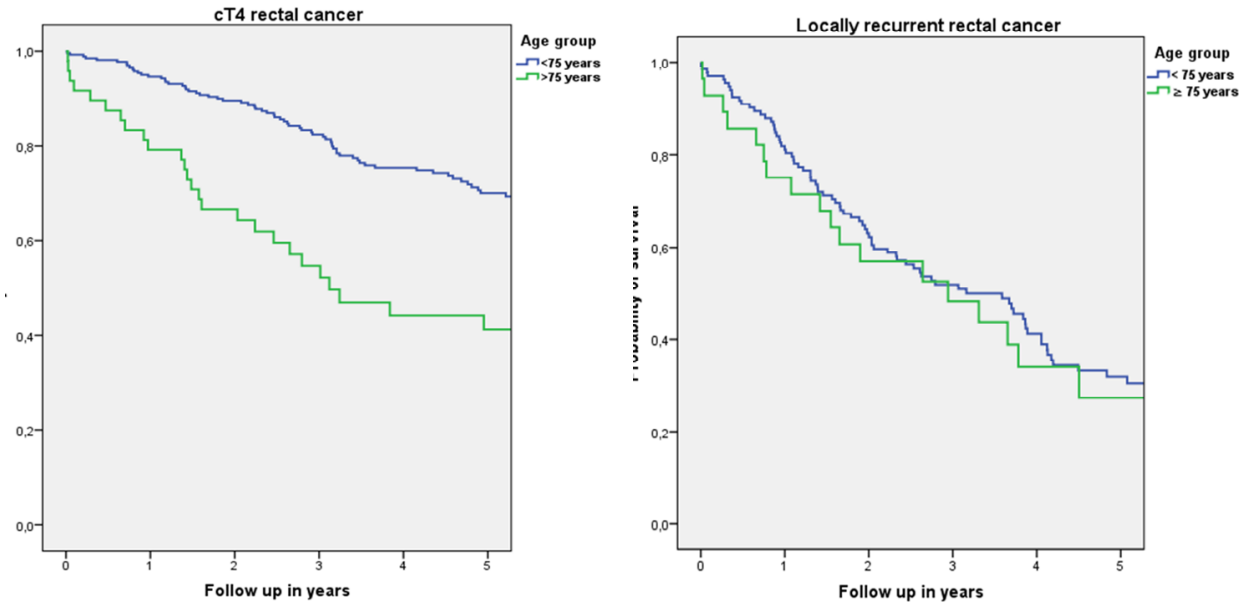

Figure 4.1 Overall survival rates of 362 cT4 and 161 LRRC patients stratified by age ( $<75$ and $\geq 75$ years) treated with curative intent in the period 2005-2013. (cT4=p<0.001 and LRRC $p=$ not significant) 
Table 4.5 Uni- and multivariate binary regression on predictive factors for one-year mortality in cT4 rectal cancer patients.

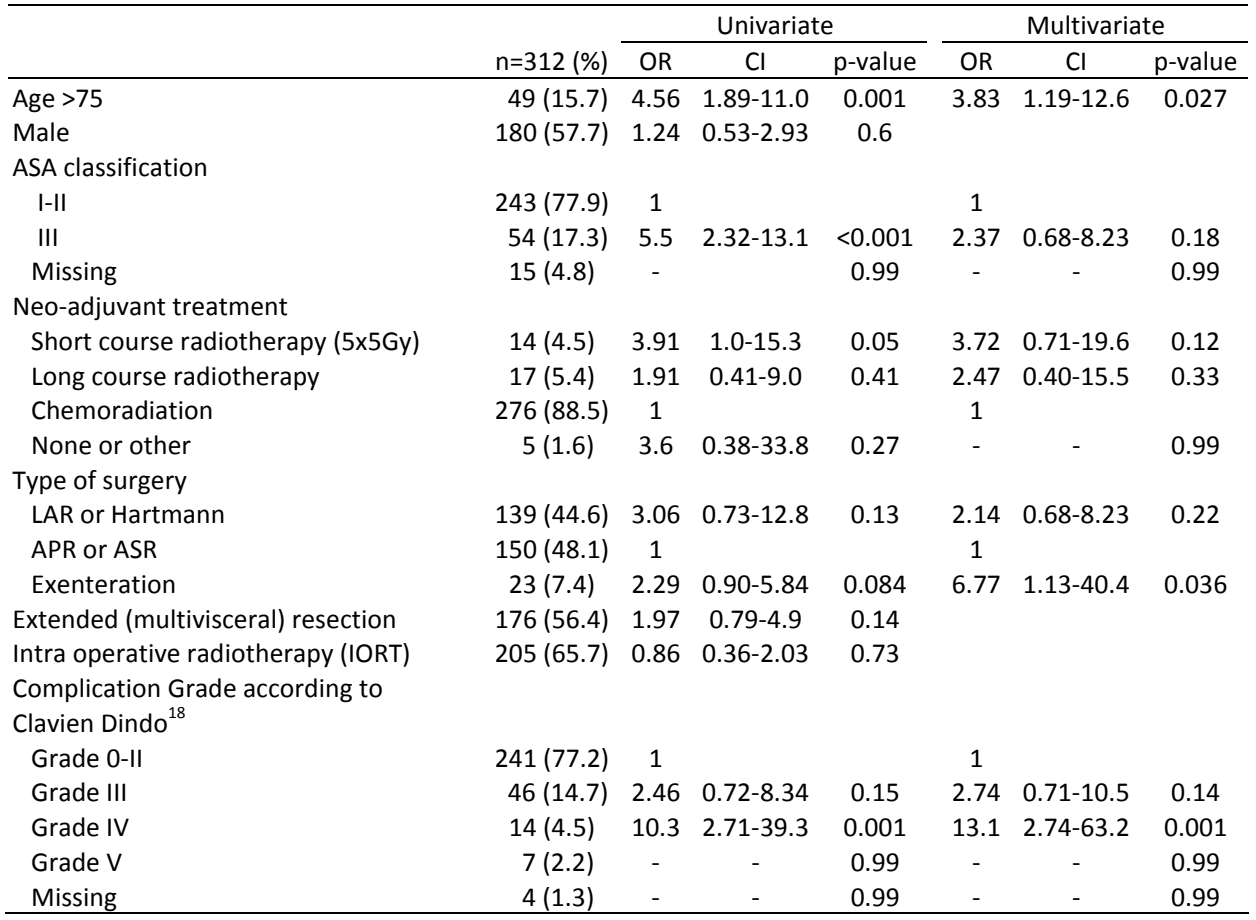

\section{Discussion}

Multimodality treatment for patients with LARC or LRRC is associated with high morbidity rates. In the current study, $56 \%$ of the cT4RC $<75$ years and $69 \%$ of the patients $\geq 75$ years had at least one complication. In LRRC, $75 \%$ of the patients $<75$ years and $96 \%$ of the patients $\geq 75$ years had at least one complication. Fortunately, the majority of the CT4RC patients had a minor complication (grade I-II) and only 12.2$15.2 \%$ of the CT4RC patients needed a radiological or surgical intervention for a grade III complication. In 4-6\% of the cT4RC patients a life threatening complication occurred. In LRRC one out of four patients $<75$ years needed an intervention for a grade III complication and a life threatening complication occurred in $4 \%$ of the cases. In elderly LRRC patients, almost half of the patients required an intervention for grade III-IV complications. These reported morbidity rates are in accordance with other studies reporting on LARC of LRRC cases with a complication $\geq$ grade III in $25 \%$ and $41 \%$ for LARC and LRRC, respectively. ${ }^{19,20}$ 
Despite the high morbidity, in hospital mortality was only $2.5 \%$ in all patients. However, it is known that particularly in elderly, the 30-day mortality rate does not predict the risk of dying during the first year post-operative year. ${ }^{4,21}$ This was also revealed in the current study with an one-year mortality rate being 2.5 and 3.5 times higher in elderly CT4RC patients. The multivariable binary regression analyses on predictive factors for one year mortality revealed that age $\geq 75$ years (OR 3.83) was a significant predictor for increased one-year mortality in CT4RC patients. Other significant factors on increased risk one-year mortality were a total exenteration and grade IV complications.

Our reported one to five-year cancer specific survival rates in elderly CT4RC patients were significantly worse compared to younger patients. There were more irradical resections in the elderly patients what could attribute to this effect. In addition, the low numbers in the elderly patient group could also attribute to this effect. Interestingly, after correction of the survival rate for one-year mortality (excluding patients who died in the first year due to complications or not tumor related death) no significant differences in long term cancer specific survival rates were seen $(p=0.079$, data not shown)

In locally recurrent rectal cancer, an underestimation of the one-year survival rate compared to the 30-day mortality rate was also observed but this was not age related. In LRRC, there is probably a mixed effect of both the more aggressive biological behaviour of the tumor and the morbidity of the treatment on survival. In order to accurately assess the effect of one-individual factor (e.g. age or treatment) on survival, larger patient sample sizes are required.

Although all patients are evaluated by both the surgeon as the anesthesiologist, specific frailty testing prior to surgery is not part of the standard care in our institute. Treatment decisions were based on patients characteristics, patients' preferences and multidisciplinary team meetings. If the surgeon or anesthesiologist suspected a poor physiological status to undergo surgery, patients were referred to a geriatrician in order to improve performance status. Due to the retrospective character of this study we were not able to extract these data from the patients' medical records, but as frailty testing was not routinely performed in our daily practice we believe that only a few patients were referred to a geriatrician. Identifying frailty in elderly patients is an important issue because it is a predictor of post-operative complications and shorter life-expectancy. ${ }^{22,23}$ Assessing frailty is most reliable performed with a Comprehensive Geriatric Assessment (CGA) tool, ${ }^{24}$ but this is time consuming and is not generally accepted for daily practice outside a geriatric clinic. A more recent risk prediction model for oncological geriatric patients with inclusion of ASA, Nutritional risk screening, Timed up and go test in combination with gender and surgery showed to have a good negative predictive value and a moderate positive predictor value for the occurrence of 
major complications. ${ }^{6}$ Another study with only rectal cancer patients found that Physiological and Operative Severity Score for enumeration of Mortality and Mortality (POSSUM) was able to predict in-hospital morbidity with an Observed/Expected (O/E) ratio of 1.05 and the Colorectal-POSSUM predicted 100-day mortality with an O/E ratio of $1.12 .^{25}$

Adequate selection of elderly patients with awareness of the morbidity of this locally progressive disease in relation to the impact of surgery on morbidity and mortality could improve outcome and quality of life. We believe that elderly patients might benefit from a pre-treatment scoring system and/or frailty testing prior to the treatment decision process. However, it is difficult to identify those patients who would benefit from pre-treatment frailty testing. In our study, 3 of the 9 of the elderly cT4RC patients who died in the first post-operative year, died due to the sequelae of complications. The other patients died due to metastatic disease $(n=3)$, complications of another surgical procedure $(n=1)$ or a death not related to treatment or disease recurrence.

A pre-treatment improvement program of the patients' physical performance and nutritional status also may improve outcome. ${ }^{26,27}$ A recent trial found meaningful changes in functional exercise capacity in colorectal patients who received exercise training and nutritional support before surgery compared to patients who received the same program after surgery on physical performance. ${ }^{28}$ Particularly in advanced cases there is enough time for optimizing the patient prior to surgery in the interval between neo-adjuvant treatment and definitive surgery. Future studies focusing on the role of a pre-habilitation program during the waiting period on post-operative outcome in elderly with advanced or recurrent cases are warranted.

The strength of this study lies in the availability of many clinically relevant variables in an unique population of patients with a low prevalence of missing values. Limitations of this study are based on its retrospective character. The report on minor complications could be underestimated due to a lack of documentation. However, we believe that the underestimation of complications was kept to a minimum by the accurate studying of medical records, contact with referral hospitals and general practitioners and direct contact with patients by telephone and validated questionnaires.

In the end, elderly patients with advanced cases should not be withheld curative treatment based on age or comorbidities alone. The use of adequate screening tools could be helpful in differentiating between those patients who benefit from curative surgery or those who benefit more from an individualised treatment without unnecessary morbidity and mortality. The effect of pre-habilitation during the neoadjuvant treatment period on morbidity and mortality should be further investigated. 


\section{Conclusion}

Morbidity after surgery for CT4RC and locally recurrent rectal cancer is high, but is regardless of age in CT4RC patients. In LRRC rectal cancer almost half of the elderly patients had at least a grade III complication. In-hospital mortality was seen in 6-7\% of the elderly CT4RC and LRRC patients. However, one month mortality in elderly CT4RC patients is a high underestimation of the risk of dying in the first post-operative year. Future studies should focus on the effect of frailty testing and pre-habilitation programs prior to surgery. 


\section{References}

1. Courtney D, McDermott F, Heeney A, Winter DC. Clinical Review: Surgical Management of Locally Advanced and Recurrent Colorectal Cancer. Langenbecks Arch Surg 2014; 399: 33-40.

2. Mohan HM, Evans MD, Larkin JO, Beynon J, Winter DC. Multivisceral Resection in Colorectal Cancer: a Systematic Review. Ann Surg Oncol 2013;20:2929-36.

3. Yang TX, Morris DL, Chua TC. Pelvic Exenteration for Rectal Cancer: a Systematic Review. Dis Colon Rectum 2013;56:519-31.

4. Dekker JW, van den Broek CB, Bastiaannet E, van de Geest LG, Tollenaar RA, Liefers GJ. Importance of the First Postoperative Year in the Prognosis of Elderly Colorectal Cancer Patients. Ann Surg Oncol 2011;18:1533-9.

5. Rutten HJ, den Dulk M, Lemmens VE, van de Velde CJ, Marijnen CA. Controversies of Total Mesorectal Excision for Rectal Cancer in Elderly Patients. Lancet Oncol 2008;9:494-501.

6. Schiphorst AH, Verweij NM, Pronk A, Hamaker ME. Age-Related Guideline Adherence and Outcome in Low Rectal Cancer. Dis Colon Rectum 2014;57:967-75.

7. Chang GJ, Skibber JM, Feig BW, Rodriguez-Bigas M. Are We Undertreating Rectal Cancer in the Elderly? An Epidemiologic Study. Ann Surg 2007;246:215-21.

8. Dharma-Wardene MW, de Gara C, Au HJ, Hanson J, Hatcher J. Ageism in Rectal Carcinoma? Treatment and Outcome Variations. Int J Gastrointest Cancer 2002;32:129-38.

9. Surgery for Colorectal Cancer in Elderly Patients: a Systematic Review. Colorectal Cancer Collaborative Group. Lancet 2000;356:968-74.

10. Balducci L. Geriatric Oncology: Challenges for the New Century. Eur J Cancer 2000;36:1741-54.

11. Dutch National Guidelines Rectal Cancer. http://www.oncoline.nl/rectalcancer . accessed 15-8-2015.

12. Martijnse IS, Dudink RL, Kusters M, et al. T3+ and T4 Rectal Cancer Patients Seem to Benefit From the Addition of Oxaliplatin to the Neoadjuvant Chemoradiation Regimen. Ann Surg Oncol 2012;19:392-401.

13. Bosman SJ, Holman FA, Nieuwenhuijzen GA, Martijn H, Creemers GJ, Rutten HJ. Feasibility of Reirradiation in the Treatment of Locally Recurrent Rectal Cancer. Br J Surg 2014;101:1280-9.

14. Bosman SJ, Vermeer TA, Dudink RL, de Hingh IH, Nieuwenhuijzen GA, Rutten HJ. Abdominosacral Resection: Long-Term Outcome in 86 Patients With Locally Advanced or Locally Recurrent Rectal Cancer. Eur J Surg Oncol 2014;40:699-705.

15. Martijnse IS, Dudink RL, West NP, et al. Focus on Extralevator Perineal Dissection in Supine Position for Low Rectal Cancer Has Led to Better Quality of Surgery and Oncologic Outcome. Ann Surg Oncol 2012; 19:786-93.

16. Dresen RC, Gosens MJ, Martijn H, et al. Radical Resection After IORT-Containing Multimodality Treatment Is the Most Important Determinant for Outcome in Patients Treated for Locally Recurrent Rectal Cancer. Ann Surg Oncol 2008;15:1937-47.

17. Mannaerts GH, Martijn H, Crommelin MA, Dries W, Repelaer van Driel OJ, Rutten HJ. Feasibility and First Results of Multimodality Treatment, Combining EBRT, Extensive Surgery, and IOERT in Locally Advanced Primary Rectal Cancer. Int J Radiat Oncol Biol Phys 2000;47:425-33.

18. Clavien PA, Barkun J, de Oliveira ML, et al. The Clavien-Dindo Classification of Surgical Complications: Five-Year Experience. Ann Surg 2009;250:187-96.

19. Berkel AE, Woutersen DP, van der Palen J, Klaase JM. Prognostic Factors for Postoperative Morbidity and Tumour Response After Neoadjuvant Chemoradiation Followed by Resection for Rectal Cancer. J Gastrointest Surg 2014;18:1648-57.

20. Alberda WJ, Verhoef C, Nuyttens JJ, et al. Outcome in Patients With Resectable Locally Recurrent Rectal Cancer After Total Mesorectal Excision With and Without Previous Neoadjuvant Radiotherapy for the Primary Rectal Tumor. Ann Surg Oncol 2014;21:520-6.

21. Visser BC, Keegan H, Martin M, Wren SM. Death After Colectomy: It's Later Than We Think. Arch Surg 2009;144:1021-7.

22. Fried LP, Tangen CM, Walston J, et al. Frailty in Older Adults: Evidence for a Phenotype. J Gerontol A Biol Sci Med Sci 2001;56:M146-M156. 
23. Kristjansson SR, Nesbakken A, Jordhoy MS, et al. Comprehensive Geriatric Assessment Can Predict Complications in Elderly Patients After Elective Surgery for Colorectal Cancer: a Prospective Observational Cohort Study. Crit Rev Oncol Hematol 2010;76:208-17.

24. Clegg A, Young J, lliffe S, Rikkert MO, Rockwood K. Frailty in Elderly People. Lancet 2013;381:752-62.

25. Stornes T, Wibe A, Endreseth BH. Complications and Risk Prediction in Treatment of Elderly Patients With Rectal Cancer. Int J Colorectal Dis 2015.

26. Fearon KC, Jenkins JT, Carli F, Lassen K. Patient Optimization for Gastrointestinal Cancer Surgery. Br J Surg 2013;100:15-27.

27. Valkenet K, van der Port, IG, Dronkers JJ, de Vries WR, Lindeman E, Backx FJ. The Effects of Preoperative Exercise Therapy on Postoperative Outcome: a Systematic Review. Clin Rehabil 2011;25:99-111.

28. Gillis C, Li C, Lee L, Awasthi R, et al. Prehabilitation Versus Rehabilitation: a Randomized Control Trial in Patients Undergoing Colorectal Resection for Cancer. Anesthesiology 2014;121:937-47. 
Health-related quality of life and sexual function after

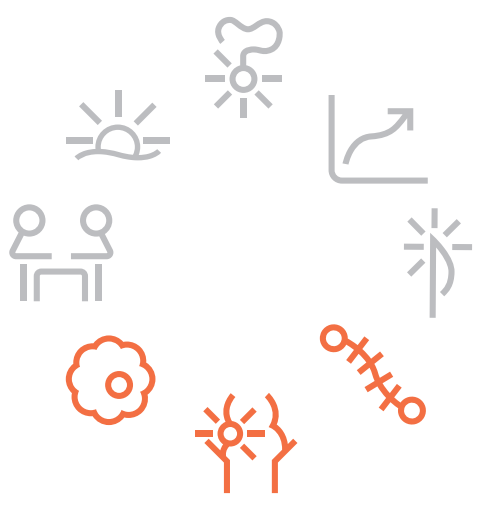





\section{CHAPTER 5}

Measuring the health-related quality of life and sexual functioning of patients with rectal cancer: does type of treatment matter?

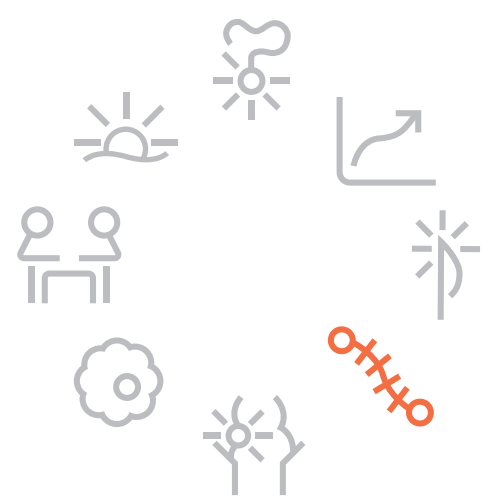

M.J. Traa

R.G. Orsini

B.L. Den Oudsten

J. De Vries J.A. Roukema

S.J. Bosman

R.L. Dudink

H.J. Rutten

Int J Cancer 


\section{Abstract}

\section{Background}

The literature on the health-related quality of life (HRQOL) after rectal cancer is growing, however, a comparison between patients with non-advanced disease (NAD), locally advanced rectal cancer (LARC), locally recurrent rectal cancer (LRRC) and a normative population has not been made. Data on the sexual functioning of patient groups is also scarce. We compared (1) the HRQOL of patients with NAD, LARC, or LRRC, with a special focus on sexual functioning and (2) the HRQOL of the three treatment groups with a normative population.

\section{Methods}

The EORTC QLQ-C30 and QLQ-CR38 were completed by 100 patients with NAD, 277 LARC patients, and 66 LRRC patients. The normative population $(n=350)$ completed the EORTC QLQ-C30 and the Sexual Functioning and Sexual Enjoyment scales of the CR38.

\section{Results}

LRRC patients reported a lower Physical Function, Social Function, Future Perspective, Sexual Functioning, and more Pain compared with LARC and NAD patients. Also, LRRC patients had a worse Body image than NAD patients and a lower Male Sexual Functioning than LARC patients. More than $75 \%$ of men and $50 \%$ of women were sexually active preoperative, compared with less than $50 \%$ and less than $35 \%$ postoperative. Male LRRC patients had more problems with erectile or ejaculatory functioning and felt less masculine than NAD or LARC patients. Women did not differ on Lubrication, Dyspareunia, and Body Image. About $10 \%$ of patients used aids in order to improve erectile functioning (men) or lubrication (women). The treatment groups reported a lower HRQOL and sexual functioning compared with the normative population.

\section{Conclusions}

This study showed that patients with LRRC reported the lowest HRQOL compared with LARC or NAD patients. All three groups reported low scores on sexual functioning compared with the normative population, while only few patients reported to used aids to improve this functioning. 


\section{Introduction}

Rectal cancer is one of the most common malignancies worldwide and has a still increasing incidence and prevalence. ${ }^{1,2}$ In $10-15 \%$ of the patients, the rectal cancer is considered locally advanced. ${ }^{3}$ In addition, $5-10 \%$ of rectal cancer patients develop a local recurrence without metastatic disease which can still be treated with a curative intent. $^{4}$

Treatment for rectal cancer is based on clinical T-stage, pathological lymph nodes and distant metastasis. ${ }^{5}$ The standard treatment for nonadvanced rectal cancer in The Netherlands is neoadjuvant radiotherapy followed by a total mesorectal excision with autonomous nerve preservation, except for cT1NO patients were radiotherapy is not indicated. ${ }^{5}$ Patients with locally advanced rectal cancer (LARC) or locally recurrent rectal cancer (LRRC) are treated with neo-adjuvant radiochemotherapy often followed by more extensive extra anatomical surgery in order to achieve a curative resection. During these procedures an intraoperative radiotherapy (IORT) boost dose can be applied at the area of risk in order to improve local control. ${ }^{6}$ The current multidisciplinary treatment for rectal cancer has led to decreased morbidity and a significant improvement of survival. ${ }^{7-9}$ However, treatment for LARC or LRRC may still be accompanied by high morbidity rates (15-68\%). ${ }^{10-13}$ Therefore, the complex and extensive treatment for LARC and LRRC can be very burdensome for the patients.

Even though the importance of patient-reported outcomes and quality of life are increasingly recognized, research on LARC or LRRC has predominantly focused on assessing local or distance control and overall survival. A recent review concluded that only seven studies evaluated the patients' health-related quality of life (HRQOL) after treatment for LARC or LRRC. ${ }^{3}$ These studies reported that the HRQL in LARC and LRRC was lower compared with patients with non-advanced disease (NAD) and normative populations (NORM). ${ }^{3,14,15}$ However, these findings were based on small sample sizes. ${ }^{3}$ Furthermore, information on sexual dysfunction is lacking. ${ }^{3,14}$ Therefore, the objectives of this cross-sectional study were (1) to compare the HRQOL of three patient populations (i.e., NAD, LARC, and LRRC), with a special focus on sexual functioning and (2) to compare the HRQOL of the three treatment groups with a normative population.

\section{Methods}

\section{Patients and procedure}

The Catharina Hospital (Eindhoven, the Netherlands) is a tertiary referral center for patients with LARC or LRRC. In the period between 2000 and 2010 a total of 
841 patients received surgery for primary rectal cancer (112 patients with a NAD and 726 patients with a LARC) and 222 patients received surgery for a LRRC. All patients who were still alive at the in $\mathbf{2 0 1 0}$ were contacted to ask them if they were willing to participate in this study. If patients agreed to participate they were asked to complete the set of standardized surveys investigating their HRQOL at home. The local ethics committees approved the study.

Normative sample data was derived from CentERdata (an online household panel) in which 1731 (81\%) members of this panel completed questionnaires. ${ }^{16}$ The description for the data collection is described elsewhere. ${ }^{16}$ For this analysis, an age and sexmatched normative population (NORM), in which a similar distribution of ages as in the patient was obtained, was included $(n=350)$. The data will be available for noncommercial scientific research, subject to study question, privacy and confidentiality restrictions, and registration (www.profilesregistry.nl). ${ }^{17}$

\section{Multidisciplinary treatment}

All tumors were identified by endoscopy and confirmed by biopsy. In addition, all patients received a preoperative magnetic resonance imaging (MRI) of the pelvis for accurate staging. A pretreatment computerized tomography (CT) was also used to exclude distant metastasis. Patients with a CT1-T3 tumor without threatened margin or pathological lymph nodes (NO) are classified with a mobile primary tumor (NAD group). Patients with a cT3+ or any CT stage with pathological lymph nodes (N1 of greater) are considered locally advanced. According to the Dutch national guidelines only patients with cT1 without positive lymph nodes are not treated with neo-adjuvant therapy. ${ }^{5}$ Patients with an LARC and distant metastasis pre-treatment who were treated with curative intent were included in the analysis. Most of the patients with NAD received short course neoadjuvant radiotherapy with $5 \times 5$ Gray (Gy). ${ }^{18}$ Most patients with LARC received neoadjuvant radiochemotherapy up to 50.4 Gy in 28 fractions to achieve downstaging in order to allow a radical resection. ${ }^{19}$ During the study period different treatment schemes of neo-adjuvant radiochemotherapy have been used. These schemes and the influence on oncological outcome have been described elsewhere. ${ }^{19}$ Finally, for LLRC, tumor growth is not confined to a specific compartment lined by fascias, because these fascias have been severed and removed during the primary surgery. This facilitates recurrent tumors to grow into surrounding compartments. However, in LRRC patients, neoadjuvant treatment options are more limited since patients already received radio(-chemo)therapy during the treatment of the primary tumor. Due to dose accumulation, toxicity tissue tolerance for re-irradiation was limited to $30 \mathrm{~Gy}{ }^{10}$

After neoadjuvant treatment most patients with LARC or LRRC received a pre-operative MRI to see whether downstaging had occurred. In almost all cases surgery was planned 
eight to 10 weeks after completion of the neoadjuvant treatment. Patients with NAD underwent either a low anterior resection with sphincter preservation and a (temporary) colostomy (LAR) or an abdominoperineal resection with permanent colostomy (APR). Patients with an advanced disease stage and involved rectal margins or patients with local recurrence were treated with a LAR or APR including extraanatomical resections in order to achieve a radical resection. An IORT boost of 10-12.5 GY was used when an involved resection margin was expected. IORT is useful in overcoming dose limitations since it can apply a dose of IORT at a well-defined specific area. Details on this multimodality treatment strategy have been described previously. ${ }^{10,20,21}$ After treatment, patients were referred to a gynecologist or an urologist when needed.

\section{Medical records}

Data were retrieved from a prospective database where information on all rectal cancer patients treated in the Catharina Hospital is registered. This information includes patient and tumor characteristics, treatment, pre-, peri-, and postoperative clinical course.

\section{Questionnaires}

Patients completed the European Organization for Research and Treatment of Cancer (EORTC) general HRQOL Questionnaire (QLQ-C30) ${ }^{22}$ and the disease specific EORTC ColoRectal 38 (QLQ-CR38). ${ }^{23}$ Both are validated for use within the Dutch population. The QLQ-C30 assesses global health status, functional health, and symptom burden, and includes a global HRQOL scale, and five scales on Physical, Role, Cognitive, Emotional and Social Functioning. It also comprises three symptoms scales on Fatigue, Nausea and Vomiting, and Pain, and six single items assessing Dyspnea, Insomnia, Loss of Appetite, Constipation, Diarrhea, and Financial Impact. The QLQ-CR38 assesses colorectal cancer specific functional health and symptom burden. It consists of scales or single items measuring Body Image, Sexual Functioning, Sexual Enjoyment, and Future Perspective. The symptom scales assess Micturition Problems, Defecation Problems, Gastrointestinal Symptoms, Stoma-related Problems, Chemotherapy Side effects, Male Sexual Functioning and Female Sexual Functioning. A single item assessed weight Loss. For both questionnaires, the items are ranged on a 4-point scale ranging from 1 (not at all) to 4 (very much) with the exception of the global health status scale, which is scored on a 7-point scale ranging from 1 (very poor) to 7 (excellent). All scales were linearly converted to a 0-100 scale according to standard scoring procedures. For the functioning scales and single items, higher scores indicate better functioning; for the symptom scales and single item, higher scores indicate higher symptom burden. 
In addition, patients were asked two additional questions. All patients were asked to what extent they were sexually active preoperatively. This question was answered on a 4-point scale: 1= As usual, 2= To a lesser extent, 3= Barely, 4= Not sexually active. In addition, men were asked if the used aids in order to get an erection. This question was answered on a 4-point scale: $1=$ No, $2=$ Yes, pills (e.g., Viagra), 3= Yes, injections, and $4=$ Yes, mechanical devices. The women were asked if they used aids in order to improve lubrication. This question was answered on a 3-point scale: $1=$ No, 2= Yes, medication (e.g., Synapauze), 3= Other aids (e.g., lubricants).

The normative sample completed the EORTC QLQ-C30 and the EORTC QLQ-CR38 subscales Sexual Functioning and Sexual Enjoyment.

\section{Statistical analyses}

Analyses of variance (ANOVA's) and chi-square tests were conducted when appropriate to present the sociodemographic and clinical characteristics for the three treatment groups (i.e., NAD, LARC, and LRRC). For patients, the EORTC-QLQ-C30 and QLQ-CR38 mean scores, stratified by group, were compared with analysis of covariance (ANCOVA's). Confounding background variables included for adjustment in these analyses were determined a priori and chosen to be age, sex, and time since surgery. The estimated marginal means were examined in order to determine which groups differed significantly from each other (a Bonferroni correction was applied). Next, similar ANCOVA's were conducted to compare the three groups with a normative population on the EORTC QLQ-C30 and EORTC QLQ-CR38 subscales Sexual Functioning and Sexual Enjoyment. However, time since surgery was no longer a confounding background variable, since this was not applicable for the normative population. In order to examine the effect of disease progression (metastasis or a not surgically treated local recurrence) secondary ANCOVA's were conducted in which patients with disease progression were excluded. Next, for the three treatment groups the scores on the sexuality questions were dichotomized in order to determine the prevalence of sexual problems. Patients who reported no problems at all or minor problems (not at all - a little bit) were categorized as not having sexual problems, while patients who reported more severe problems (quite some - very much) were categorized as having sexual problems. In addition, questions regarding sexual activity were dichotomized into 0 (not sexually active) or 1 (sexually active). Furthermore, the scores on the aids questions were dichotomized into 0 (no resources used) and 1 (resources used). Means and standard deviations are provided as $(M \pm S D)$. Statistical differences were indicated if $p<.05$ (two-sided). All statistical analyses were performed using SPSS17.0. 


\section{Results}

\section{Study population}

In total, 80 patients with NAD, 292 patients with LARC, 67 patients with LRRC returned the completed questionnaire and were included in the current study. This reflected an $85 \%$ response rate. The sociodemographic and clinical characteristics of the patients are presented in Table 5.1. The patients with NAD patients mostly received neoadjuvant short course radiotherapy (86.3\%) while patients with LARC received neoadjuvant chemoradiation (84.6\%). Patients with LRRC received neo-adjuvant reirradiation combined with chemotherapy $(56.7 \%)$ or full course chemoradiation (37.3\%). In addition, type of surgery differed per group. In the NAD group a LAR was more often performed (67.5\%) than an APR (28.8\%), for the LARC group these percentages were more equal (47.6\% versus $43.2 \%$, respectively). As can be expected, the percentage of multivisceral resections was higher in the LRRC group (53.7\%) than the LARC (39\%) and NAD group (1.6\%). The normative sample consisted of 200 men

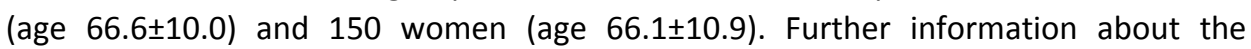
normative sample is published elsewhere. ${ }^{24,25}$

\section{Scores on the EORTC QLQ-C30 and EORTC QLQ-CR38}

After adjusting for age, sex, and time since diagnosis, LRRC patients reported a lower Physical Function, Social Function, Future Perspective, Sexual Functioning and more Pain compared with LARC patients and patients with NAD ( $p^{\prime} s<0.05$, see Table 5.2 ). Furthermore, LRRC patients experienced a worse Body image compared with NAD patients $(p=0.016)$ and a lower Male Sexual Functioning compared with LARC patients $(p=0.018)$. Excluding patients with a disease progression resulted in similar results, with exception of the Male Sexual Functioning and Future Perspective scale for which the differences were no longer statistically significant (results not shown).

In-depth analyses of the sexuality items revealed that more than $75 \%$ of men were sexually active preoperative, regardless of their treatment $(p=0.397$, see Table 5.3$)$. After treatment, these percentages dropped to less than 50\%. LRRC patients more often had problems with erectile functioning and felt less masculine (see Table 5.4) compared with NAD or LARC patients $\left(p^{\prime} s<0.05\right)$. However, less than $10 \%$ of the patients used aids to enhance erectile functioning ( $p=0.610)$. More than $50 \%$ of women were sexually active preoperative. However, after treatment $34.3 \%$ of NAD, $23.0 \%$ of LARC, and $26.1 \%$ of LRRC patients were still sexually active $(p=0.427)$. Women did not differ on Lubrication, Dyspareunia, and their Body Image. Less than $15 \%$ of women used aids to enhance lubrication. However, more than $50 \%$ of women did not answer the questions on lubrication, dyspareunia, and aids used to enhance lubrication. 
Table 5.1 Demographic and clinical characteristics of the three treatment groups.

\begin{tabular}{|c|c|c|c|}
\hline & $\begin{array}{c}\text { NAD } \\
(n=80)\end{array}$ & $\begin{array}{c}\text { LARC } \\
(n=292)\end{array}$ & $\begin{array}{c}\text { LRRR } \\
(n=67)\end{array}$ \\
\hline Age at time of survey (mean $\pm S D$ ) & $70.4 \pm 9.7$ & $65.6 \pm 9.7$ & $63.7 \pm 9.8$ \\
\hline \multirow{2}{*}{ Years since surgery (median (range)) } & $4.5(0-11)$ & $2.3(0-11)$ & $3.3(0-10)$ \\
\hline & $\mathrm{N}(\%)$ & $\mathrm{N}(\%)$ & $N(\%)$ \\
\hline Male (yes) & $46(57.5)$ & 169 (57.9) & $44(65.7)$ \\
\hline \multicolumn{4}{|l|}{ Neoadjuvant treatment } \\
\hline No neoadjuvant treatment & $10(132.5)$ & $1(0.3)$ & $2(3)$ \\
\hline Short course radiotherapy (5x5 Gy) & $69(86.3)$ & $18(6.2)$ & NA \\
\hline Long course radiotherapy & & $26(8.9)$ & NA \\
\hline Chemoradiaton & $1(1.3)$ & $247(84.6)$ & NA \\
\hline Reirradiation with chemotherapy & NA & NA & $38(56.7)$ \\
\hline Reirradiation without chemotherapy & NA & NA & $1(1.5)$ \\
\hline Full course irradiation with chemotherapy & NA & NA & $25(37.3)$ \\
\hline Full course irradiation & NA & NA & $1(1.5)$ \\
\hline \multicolumn{4}{|l|}{ Type of surgery } \\
\hline Low anterior resection & $54(67.5)$ & $139(47.6)$ & 8 (11.9) \\
\hline Abdominoperineal resection & $23(28.8)$ & $126(43.2)$ & $15(22.4)$ \\
\hline Abdominosacral resection & - & $5(1.7)$ & $19(28.4)$ \\
\hline Exenteration (Anterior/Pelvic) & - & $11(3.8)$ & $9(13.4)$ \\
\hline Hartmann & $3(3.8)$ & $11(3.8)$ & $3(4.5)$ \\
\hline Other & - & $1(0.4)$ & 0 \\
\hline Multivisceral (incl. prostate/uterus/vesiculae) & $2(1.6)$ & $114(39.0)$ & $31(53.7)$ \\
\hline Intra operative radiotherapy (IORT) & - & $189(64.7)$ & $58(86.6)$ \\
\hline \multicolumn{4}{|l|}{ Radical treatment } \\
\hline Radical & $78(97.5)$ & $265(90.8)$ & $51(76.1)$ \\
\hline Microscopic focal irradical & - & $26(8.9)$ & $16(23.9)$ \\
\hline Missing & $2(2.5)$ & $1(0.3)$ & 0 \\
\hline Not surgically treated Local Recurrence & 0 & $6(2.1)$ & 0 \\
\hline Missing & - & - & \\
\hline Distant Metastases & $2(2.5)$ & $33(11.3)$ & $11(16.4)$ \\
\hline Missing & - & - & 0 \\
\hline \multicolumn{4}{|l|}{ Clinical T stage } \\
\hline $\mathrm{T} 1-2$ & $40(50.0)$ & $3(1.0)$ & \\
\hline T3 & $40(50.0)$ & $107(36.6)$ & \\
\hline $\mathrm{T} 4$ & - & $177(60.6)$ & \\
\hline Missing & - & $5(1.7)$ & \\
\hline \multicolumn{4}{|l|}{ Clinical N stage } \\
\hline NO & $80(100)$ & $97(33.2)$ & - \\
\hline N1 & - & $92(31.5)$ & - \\
\hline N2 & - & $59(20.2)$ & - \\
\hline Missing & - & $44(15.1)$ & - \\
\hline Clinical M1 stage & - & $15(5.3)$ & - \\
\hline \multicolumn{4}{|l|}{ Postoperative abdominal complications } \\
\hline Presacral abscess & $10(12.5)$ & $25(8.6)$ & $5(7.5)$ \\
\hline Anastomic leakage & $3(3.8)$ & $15(4.1)$ & $2(3)$ \\
\hline
\end{tabular}


Table 5.2 Mean scores on the EORTC QLQ-C30 and QLQ-CR38 of the three treatment groups.

\begin{tabular}{|c|c|c|c|c|c|c|}
\hline & $\begin{array}{c}\text { NAD } \\
(n=80)\end{array}$ & $\begin{array}{c}\text { LARC } \\
(n=292)\end{array}$ & $\begin{array}{c}\text { LRRC } \\
(n=67)\end{array}$ & p-value** & Norm. Pop & $\mathrm{p}$-value*** \\
\hline \multicolumn{7}{|l|}{ EORTC C30 subscales } \\
\hline Global quality of life* & $70.5 \pm 22.1$ & $68.1 \pm 23.4$ & $64.0 \pm 24.0$ & 0.313 & $77.2 \pm 17.4$ & $<0.0001$ \\
\hline Physical function* & $80.3 \pm 19.1$ & $79.5 \pm 20.4$ & $71.9 \pm 22.8$ & 0.022 & $86.0 \pm 17.1$ & $<0.0001$ \\
\hline Role function* & $75.0 \pm 28.4$ & $75.3 \pm 28.1$ & $69.6 \pm 31.6$ & 0.315 & $86.4 \pm 21.6$ & $<0.0001$ \\
\hline Emotional function* & $84.8 \pm 18.8$ & $82.6 \pm 20.7$ & $81.1 \pm 22.6$ & 0.630 & $90.3 \pm 15.0$ & $<0.0001$ \\
\hline Cognitive function* & $84.4 \pm 20.9$ & $83.7 \pm 21.1$ & $80.9 \pm 22.1$ & 0.498 & $90.8 \pm 14.1$ & $<0.0001$ \\
\hline Social function* & $82.7 \pm 22.4$ & $79.2 \pm 25.2$ & $69.6 \pm 29.2$ & 0.009 & $93.1 \pm 16.8$ & $<0.0001$ \\
\hline Fatigue ${ }^{\dagger}$ & $23.1 \pm 22.3$ & $26.8 \pm 23.9$ & $26.2 \pm 22.1$ & 0.526 & $17.8 \pm 20.5$ & $<0.0001$ \\
\hline Nausea / vomiting ${ }^{\dagger}$ & $3.0 \pm 8.3$ & $4.4 \pm 11.8$ & $4.0 \pm 11.6$ & 0.736 & $3.0 \pm 11.8$ & 0.311 \\
\hline Paint & $14.8 \pm 21.3$ & $15.8 \pm 22.8$ & $41.9 \pm 22.3$ & $<0.0001$ & $17.8 \pm 23.0$ & $<0.0001$ \\
\hline Dyspnoeat & $16.0 \pm 24.4$ & $13.8 \pm 22.5$ & $12.4 \pm 19.3$ & 0.613 & $9.4 \pm 19.6$ & 0.028 \\
\hline Insomnia† & $19.4 \pm 27.5$ & $18.7 \pm 26.5$ & $21.3 \pm 30.4$ & 0.771 & $14.9 \pm 22.0$ & 0.067 \\
\hline Appetite loss ${ }^{\dagger}$ & $3.8 \pm 10.7$ & $6.7 \pm 17.7$ & $3.2 \pm 9.9$ & 0.194 & $3.3 \pm 12.0$ & 0.019 \\
\hline Constipation $t^{\S}$ & $8.4 \pm 16.4$ & $9.1 \pm 20.5$ & $9.3 \pm 23.7$ & 0.951 & $5.6 \pm 15.3$ & 0.037 \\
\hline Diarrhoeat $t^{\S}$ & $21.5 \pm 27.8$ & $18.7 \pm 25.3$ & $19.4 \pm 26.0$ & 0.512 & $4.5 \pm 15.1$ & $<0.0001$ \\
\hline Financial problems ${ }^{\dagger}$ & $5.6 \pm 17.3$ & $11.9 \pm 23.3$ & $8.2 \pm 19.9$ & 0.436 & $3.0 \pm 12.0$ & $<0.0001$ \\
\hline \multicolumn{7}{|l|}{ EORTC CR38 subscales } \\
\hline Body image* & $80.1 \pm 22.8$ & $73.5 \pm 26.4$ & $65.5 \pm 29.9$ & 0.016 & - & - \\
\hline Future perspective* & $71.4 \pm 26.2$ & $66.7 \pm 27.3$ & $57.2 \pm 34.2$ & 0.018 & - & - \\
\hline Sexual Functioning* & $25.5 \pm 27.1$ & $21.6 \pm 24.8$ & $15.2 \pm 19.5$ & 0.009 & $31.4 \pm 25.5$ & $<0.0001$ \\
\hline Sexual Enjoyment* & $58.1 \pm 25.8$ & $56.8 \pm 27.7$ & $66.7 \pm 26.1$ & 0.471 & $64.3 \pm 25.1$ & $<0.0001$ \\
\hline Micturition problems ${ }^{\dagger}$ & $25.3 \pm 17.2$ & $24.9 \pm 16.3$ & $25.1 \pm 17.9$ & 0.945 & - & - \\
\hline Chemotherapy side effects ${ }^{\dagger}$ & $11.0 \pm 13.4$ & $14.7 \pm 17.4$ & $11.1 \pm 12.7$ & 0.228 & - & - \\
\hline Gastro-intestinal problems ${ }^{\dagger}$ & $17.3 \pm 15.4$ & $20.3 \pm 14.8$ & $21.7 \pm 14.7$ & 0.381 & - & - \\
\hline Male Sexual Functioning ${ }^{\dagger}$ & $69.9 \pm 31.3$ & $69.3 \pm 32.5$ & $86.3 \pm 23.2$ & 0.014 & - & - \\
\hline Female Sexual functioning ${ }^{\dagger}$ & $29.5 \pm 27.3$ & $37.2 \pm 33.9$ & $41.7 \pm 41.9$ & 0.747 & - & - \\
\hline Stoma-related problems ${ }^{\dagger}$ & $23.2 \pm 22.4$ & $25.7 \pm 18.8$ & $24.5 \pm 19.6$ & 0.987 & - & - \\
\hline Weight losst & $8.0 \pm 18.7$ & $10.8 \pm 21.4$ & $9.1 \pm 18.3$ & 0.711 & - & - \\
\hline
\end{tabular}

* Higher scores indicate better functioning. HHigher scores indicate more problems. Norm.pop = Normative population ${ }^{* *}$ p-value for the comparison between the three treatment groups, adjusted for age, sex, and time since diagnosis. ${ }^{* *}$ p-value for the comparison between all four groups, adjusted for age and sex. The mean scores on the subscale Defecation Problems are not computed because this is not applicable for most patients since they have a stoma.

\section{Comparison with a normative population}

Compared with the normative population, rectal cancer patients had lower scores on Global Quality of Life, Role Function, Cognitive Function, Emotional Function, Social Function, and Sexual Enjoyment, and more Constipation and Diarrhea, regardless of treatment ( $p^{\prime} s<0.05$; see Table 5.2). In addition, LARC and LRRC patients experienced a lower Physical Function, Sexual Functioning but more Fatigue than the normative population ( $\left.p^{\prime} s<0.05\right)$. Finally, LRRC patients reported more Pain $(p<0.0001)$ than the normative population, while the LARC group reported more Appetite Loss ( $p=0.017)$, Dyspnoea $(p=.026)$, and Financial problems $(p<0.0001)$ compared with the normative population. Excluding patients with a disease progression resulted in similar results (results not shown). 
Table 5.3 The effect of treatment on sexual functioning for men and women.

\begin{tabular}{|c|c|c|c|c|c|c|c|c|}
\hline & & MEN & & & & WOMEN & & \\
\hline & $\begin{array}{c}\text { NAD } \\
(n=45)\end{array}$ & $\begin{array}{l}\text { LARC } \\
(\mathrm{n}=170)\end{array}$ & $\begin{array}{r}\text { LRRC } \\
(n=44)\end{array}$ & $\mathrm{p}$-value & $\begin{array}{c}\text { NAD } \\
(n=35)\end{array}$ & $\begin{array}{l}\text { LARC } \\
(n=122)\end{array}$ & $\begin{array}{r}\text { LRRC } \\
(n=23)\end{array}$ & $p$-value \\
\hline $\begin{array}{l}\text { Sexual active } \\
\text { preoperative }\end{array}$ & & & & 0.397 & & & & 0.071 \\
\hline Yes & 42 (93.3\%) & $141(82.9 \%)$ & 35 (79.5\%) & & $24(68.6 \%)$ & 75 (61.5\%) & $12(52.2 \%)$ & \\
\hline No & $2(4.4 \%)$ & 17 (10.0\%) & 5 (11.4\%) & & 5 (14.3\%) & 16 (13.1\%) & $8(34.8 \%)$ & \\
\hline Missing & $1(2.2 \%)$ & 12 (7.1\%) & $4(9.1 \%)$ & & $6(17.1 \%)$ & 31 (25.4\%) & $3(13.0 \%)$ & \\
\hline $\begin{array}{l}\text { Sexually active } \\
\text { postoperative }\end{array}$ & & & & 0.054 & & & & 0.427 \\
\hline Yes & 21 (46.7\%) & 75 (44.1\%) & $11(25.0 \%)$ & & $12(34.3 \%)$ & $28(23.0 \%)$ & 6 (26.1\%) & \\
\hline No & 23 (51.1\%) & 93 (54.7\%) & 32 (72.7\%) & & $17(48.6 \%)$ & 70 (57.4\%) & 13 (56.5\%) & \\
\hline Missing & $1(2.2 \%)$ & $2(1.2 \%)$ & $1(2.3 \%)$ & & $6(17.1 \%)$ & $24(19.7 \%)$ & $4(17.4 \%)$ & \\
\hline $\begin{array}{l}\text { Erectile functioning }(\delta) / \\
\text { Lubrication ( }(+)\end{array}$ & & & & 0.010 & & & & 0.571 \\
\hline No problems & $7(15.6 \%)$ & 39 (22.9\%) & $2(4.5 \%)$ & & $10(28.6 \%)$ & $33(27.0 \%)$ & $3(13.0 \%)$ & \\
\hline $\begin{array}{l}\text { Problems to some } \\
\text { extent }\end{array}$ & 31 (68.9\%) & $113(66.5 \%)$ & $41(93.2 \%)$ & & $5(14.3 \%)$ & 24 (19.7\%) & $4(17.4 \%)$ & \\
\hline Missing & $7(15.6 \%)$ & $18(10.6 \%)$ & 1 (2.3\%) & & 20 (57.1\%) & 65 (53.3\%) & 16 (69.6\%) & \\
\hline $\begin{array}{l}\text { Ejaculatory functioning } \\
\left({ }^{\lambda}\right) \text { / Dyspareunia }(+)\end{array}$ & & & & 0.059 & & & & 0.235 \\
\hline No problems & $11(24.4 \%)$ & $50(29.4 \%)$ & $6(13.6 \%)$ & & $14(40.0 \%)$ & 37 (30.3\%) & $2(8.7 \%)$ & \\
\hline $\begin{array}{l}\text { Problems to some } \\
\text { extent }\end{array}$ & $26(57.8 \%)$ & 98 (57.6\%) & 35 (79.5\%) & & $2(5.7 \%)$ & 15 (12.3\%) & $2(8.7 \%)$ & \\
\hline Missing/NA & $8(17.8 \%)$ & 22 (12.9\%) & $3(6.8 \%)$ & & 19 (54.3\%) & 70 (57.4\%) & 19 (82.6\%) & \\
\hline $\begin{array}{l}\text { Aids used to enhance } \\
\text { erectile functioning* }\end{array}$ & & & & 0.610 & & & & 0.703 \\
\hline No & 37 (82.2\%) & $131(77.1 \%)$ & $36(81.8 \%)$ & & 15 (42.9\%) & $50(41.0 \%)$ & 5 (21.7\%) & \\
\hline Yes & $2(4.4 \%)$ & $15(8.8 \%)$ & $4(9.1 \%)$ & & $5(14.3 \%)$ & $10(8.2 \%)$ & $1(4.3 \%)$ & \\
\hline Missing & $6(13.3 \%)$ & 24 (14.1\%) & 4 (9.1\%) & & 15 (42.9\%) & $62(50.8 \%)$ & 17 (73.9\%) & \\
\hline
\end{tabular}

In both tables the scores on the individual EORTC QLQ-CR38 items were dichotomized into two categories (not at all $-\mathrm{a}$ bit $=0$ and some problems - very much problems $=1$ ).

\section{Discussion}

Patients with a LRRC reported a lower HRQOL compared with both LARC patients and patients with NAD. These results are consistent with the results of Palmer et al. who described that patients with an extensive disease had a lower HRQL compared with patients with NAD. ${ }^{14}$ However, they pooled the LARC and LRRC patients in one group, while our study clearly shows that the LRRC group experiences a lower HRQOL compared with the LARC group. This could be due to the fact that patients with a LRRC were treated with more extensive treatment to achieve radical surgery (e.g., extra anatomical resections), which may have influenced the physical condition and psychological state of the patient. In this study no significant differences between the LARC and NAD groups were found. The study of Austin et al. also reported that patients 
with pelvic exenteration did not differ on physical, social, emotional, and functional well-being compared with patients who underwent a low anterior resection or an abdominoperineal resection. ${ }^{15}$ Compared with a Dutch normative population all three groups had a lower HRQOL, which is in line with earlier studies. ${ }^{14,15}$

Table 5.4 The effect of treatment on body image for men and women.

\begin{tabular}{|c|c|c|c|c|c|c|c|c|}
\hline & \multicolumn{3}{|c|}{ MEN } & \multicolumn{5}{|c|}{ WOMEN } \\
\hline & $\begin{array}{c}\text { NAD } \\
(n=45)\end{array}$ & $\begin{array}{c}\text { LARC } \\
(n=170)\end{array}$ & $\begin{array}{c}\text { LRRC } \\
(n=44)\end{array}$ & $\mathrm{p}$-value & $\begin{array}{c}\text { NAD } \\
(n=35)\end{array}$ & $\begin{array}{c}\text { LARC } \\
(n=122)\end{array}$ & $\begin{array}{c}\text { LRRC } \\
(n=23)\end{array}$ & $\mathrm{p}$-value \\
\hline Feeling less attractive & & & & .061 & & & & .460 \\
\hline \multicolumn{9}{|l|}{ due to disease/treatment } \\
\hline Yes & $4(8.9 \%)$ & 27 (15.9\%) & $12(27.3 \%)$ & & $6(17.1 \%)$ & $28(23.0 \%)$ & $3(13.0 \%)$ & \\
\hline No & $41(91.1 \%)$ & $142(83.5 \%)$ & $32(72.2 \%)$ & & $29(82.9 \%)$ & $90(73.8 \%)$ & $19(82.6 \%)$ & \\
\hline Missing & $0(0.0 \%)$ & $1(0.6 \%)$ & $0(0.0 \%)$ & & $0(0.0 \%)$ & $4(3.3 \%)$ & $1(4.3 \%)$ & \\
\hline Feeling less & & & & .046 & & & & .526 \\
\hline \multicolumn{9}{|l|}{ masculine/feminine } \\
\hline Yes & $11(24.4 \%)$ & $46(27.1 \%)$ & $20(45.5 \%)$ & & $3(8.6 \%)$ & $20(16.4 \%)$ & $3(13.0 \%)$ & \\
\hline No & $33(73.3 \%)$ & $123(72.4 \%)$ & $24(54.5 \%)$ & & $30(85.7 \%)$ & $98(80.3 \%)$ & $19(82.6 \%)$ & \\
\hline Missing & $1(2.2 \%)$ & $1(0.6 \%)$ & $0(0.0 \%)$ & & $2(5.7 \%)$ & $4(3.3 \%)$ & $1(4.3 \%)$ & \\
\hline Dissatisfied with body & & & & .723 & & & & .262 \\
\hline Yes & $9(20.0 \%)$ & $34(20.0 \%)$ & $11(25.0 \%)$ & & $5(14.3 \%)$ & $30(24.6 \%)$ & $7(30.4 \%)$ & \\
\hline No & $36(80.0 \%)$ & $135(79.4 \%)$ & $32(72.7 \%)$ & & $29(82.9 \%)$ & $90(73.8 \%)$ & 14 (60.9\%) & \\
\hline Missing & $0(0.0 \%)$ & $1(0.6 \%)$ & $1(2.3 \%)$ & & $1(2.9 \%)$ & $2(1.6 \%)$ & $2(8.7 \%)$ & \\
\hline
\end{tabular}

In both tables the scores on the individual EORTC QLQ-CR38 items were dichotomized into two categories (not at all $-\mathrm{a}$ bit $=0$ and some - very much problems $=1$ ).

Compared with the preoperative percentages, the percentage of patients that were still sexually active at time of questionnaire completion had dropped dramatically. For men, more than $75 \%$ of patients were sexually active preoperative compared with less than $50 \%$ postoperative. For women, these percentages were lower; more than $50 \%$ of women were sexually active preoperative compared with less than $35 \%$ postoperative. Moreover, patients with LRRC or LARC reported a lower Sexual Functioning than NAD patients and LRRC patients reported a worse Male Sexual Functioning than LARC patients. Furthermore, LRRC patients most often experienced problems with erectile functioning and they more often reported to feel less masculine. For women, no differences were reported for lubrication problems, dyspareunia, and body image. The substantial decline in the percentage of sexually active participants could also partly reflect normal effects of aging, since the mean time since surgery was 2.0 (range 0-11 years). However, the fact remains that all three groups reported a lower sexual functioning (which includes sexual activity) and sexual enjoyment compared with the normative population. Based on these results it may be expected that age does not fully explain the lower levels of functioning and sexual enjoyment in our population.

Even though a high prevalence of sexual dysfunction was found, less than $15 \%$ of patients reported to use aids in order to improve erectile functioning (men) or 
lubrication (women). The discrepancy between the presence of sexual dysfunction and the frequency by which aids are used in an attempt to improve sexual functioning is remarkable. These numbers may be due to an insufficient knowledge of patients about the possible treatments for sexual dysfunction. Previous studies have already shown that although it was important for most of the patients to discuss sexuality, these discussions do not always occur. ${ }^{26,27}$ Furthermore, not all patients may feel inclined to seek help for their problems, perhaps they have found other ways to maintain a satisfactory relationship. There may be a still existing taboo on discussing sexuality, in both health care professionals as patients. Psychosexual education before and after treatment may play an important role in increasing the patients' knowledge. In this way, professionals normalize patients' concerns, which may lower the threshold for patients to seek adequate support. Furthermore, it is imperative that health care professionals adequately assess, evaluate, and manage potential sexual problems.

The merits of the current study are the fact that this study achieved a high response rate $(85 \%)$. To our knowledge this is the first study to investigate the HRQOL in NAD, LARC, and LRRC. In addition, the comparison of these three groups with a normative population was not yet performed. Finally, we used validated and reliable questionnaires. However, there are also some limitations that need to be acknowledged. For instance, the cross-sectional nature of this study presents an inherent limitation. Furthermore, no information was known about the patients' functioning before treatment, except of knowledge on whether or not patients were sexually active preoperative, which limits the determination of the effect of a cancer diagnosis and treatment on functioning. In addition, it is possible that the question on preoperative sexual activity was not clear for the LRRC group. It is a possibility that the LRRC group thought that the question referred to their sexual activity prior to the first surgery (for the primary cancer) instead of the second surgery, which may explain the remarkable difference between the percentage of postoperative sexual active patients in the NAD group and the preoperative sexual active patients in the LRRC group. Furthermore, even though the EORTC QLQ-CR38 is one of the most used questionnaires to assess sexual functioning, it provides limited information since sexual functioning is assessed with only five questions. Finally, more than $50 \%$ of women did not answer the questions regarding lubrication, dyspareunia, and aids used to enhance lubrication. This may be attributed to the way these questions are postulated. The questions "Did you have a dry vagina during intercourse" and "Did you have pain during intercourse" imply that a women needs to be sexually active in order to encounter possible problems. However, women may not be sexually active due to these problems; therefore, the causality implied is not correct. Future research should use questionnaires designed to assess sexuality more adequately. In addition, according to the Dutch national guideline only patients with cT1NO are not treated with neoadjuvant treatment. ${ }^{5}$ In our study only 8 patients with a NAD had a cT1NO tumor and did therefore not receive 
neoadjuvant radiotherapy. However, negative effects of neoadjuvant radiotherapy on HRQOL has been previously reported. ${ }^{28,29}$ The fact that almost all patients received neoadjuvant treatment may therefore, to some extent, explain the lack of differences in HRQOL between the LARC and NAD group. In addition, with the introduction of the new guidelines for rectal cancer treatment patients with CT2NO rectal cancers will no longer be eligible for neoadjuvant radiotherapy. Therefore, future studies will be able to examine two subgroups within NAD: those who received neoadjuvant treatment (cT3NO) and those who did not (cT1NO or CT2NO). In this way, the potential effects of neoadjuvant radiotherapy in patients with NAD can be evaluated more specifically. Furthermore, in the Catharina hospital patients receive IORT when an involved surgical margin can be expected. Multiple studies about the effectiveness of IORT in LARC patients are performed. ${ }^{7,30,31}$ These studies report an additional benefit of IORT on local control and outcome. However, IORT may have a negative impact on HRQOL, particularly on the sexuality and uro-genital items. In this study only minor differences between the NAD and LARC patients were found. Nevertheless, future studies are still warranted to examine the effects of IORT on HRQOL more specifically. Finally, since our study population was already stratified into three groups (NAD, LARC, LRRC) it was no possible to conduct the analyses specifically for patients with a stoma or to include stoma as a covariate as this would, unfortunately, have resulted in too low sample sizes. Therefore, it would be beneficial if upcoming studies provide further insights in the influence of a (temporary) stoma on (HR)QOL.

\section{Conclusion}

This study showed that patients with LRRC reported the lowest HRQOL compared with LARC or NAD patients. All three groups reported low scores on sexual functioning compared with the normative population, while only few patients reported to used aids to improve this functioning. 


\section{References}

1. Project TG. Estimated cancer incidence, mortality, prevalence and disability-adjusted life years (DALYS) Worldwide in 2008, 2008.

2. Cancer in the Netherlands. Trends, prognoses and implications for health care. (kanker in Nederland. Trends, prognoses, en implicaties voor zorgvraag)ed. Amsterdam, KWF KankerbestrijdingSignaleringscommissie Kanker, 2004.

3. Thaysen HV, Jess P, Laurberg S. Health-Related Quality of Life after surgery for primary advanced rectal cancer and recurrent rectal cancer: a review. Colorectal Dis 2012;14:797-803.

4. den Dulk M, van de Velde CJ. Quality assurance in surgical oncology: the tale of the Dutch rectal cancer TME trial. J Surg Oncol 2008;97:5-7.

5. www.oncoline.nl/rectalcancer Accessed June 14, 2013

6. Kusters M, Holman FA, Martijn H, Nieuwenhuijzen GA, Creemers GJ, Daniels-Gooszen AW, van den Berg $\mathrm{HA}$, van den Brule AJ, van de Velde CJ, Rutten HJ. Patterns of local recurrence in locally advanced rectal cancer after intra-operative radiotherapy containing multimodality treatment. Radiother Oncol 2009; 92:221-5.

7. Kusters M, Valentini V, Calvo FA, Krempien R, Nieuwenhuijzen GA, Martijn H, Doglietto GB, Del Valle E, Roeder F, Buchler MW, van de Velde CJ, Rutten HJ. Results of European pooled analysis of IORTcontaining multimodality treatment for locally advanced rectal cancer: adjuvant chemotherapy prevents local recurrence rather than distant metastases. Ann Oncol 2010;21:1279-84.

8. den Dulk M, Krijnen P, Marijnen CA, Rutten HJ, van De Poll-Franse LV, Putter H, Meershoek-Klein Kranenbarg $\mathrm{E}$, Jansen-Landheer $\mathrm{ML}$, Coebergh JW, van de Velde C. Improved overall survival for patients with rectal cancer since 1990: the effects of TME surgery and pre-operative radiotherapy. Eur J Cancer 2008;44:1710-16.

9. Wiig JN, Larsen SG, Dueland S, Giercksky KE. Clinical outcome in patients with complete pathologic response (pT0) to preoperative irradiation/chemo-irradiation operated for locally advanced or locally recurrent rectal cancer. J Surg Oncol 2005;92:70-5.

10. Dresen RC, Gosens MJ, Martijn H, Nieuwenhuijzen GA, Creemers GJ, Daniels-Gooszen AW, van den Brule AJ, van den Berg HA, Rutten HJ. Radical resection after IORT-containing multimodality treatment is the most important determinant for outcome in patients treated for locally recurrent rectal cancer. Ann Surg Oncol 2008;15:1937-47.

11. Peeters KC, Tollenaar RA, Marijnen CA, Klein Kranenbarg E, Steup WH, Wiggers T, Rutten HJ, van de Velde CJ. Risk factors for anastomotic failure after total mesorectal excision of rectal cancer. Br J Surg 2005;92:211-6.

12. Rahbari NN, Ulrich AB, Bruckner T, Munter M, Nickles A, Contin P, Loffler T, Reissfelder C, Koch M, Buchler MW, Weitz J. Surgery for locally recurrent rectal cancer in the era of total mesorectal excision: is there still a chance for cure? Ann Surg 2011;253:522-33.

13. Vermaas M, Ferenschild FT, Verhoef C, Nuyttens JJ, Marinelli AW, Wiggers T, Kirkels WJ, Eggermont AM, de Wilt JH. Total pelvic exenteration for primary locally advanced and locally recurrent rectal cancer. Eur J Surg Oncol 2007;33:452-8.

14. Palmer G, Martling A, Lagergren P, Cedermark B, Holm T. Quality of life after potentially curative treatment for locally advanced rectal cancer. Ann Surg Oncol 2008;15:3109-17.

15. Austin KK, Young JM, Solomon MJ. Quality of life of survivors after pelvic exenteration for rectal cancer. Dis Colon Rectum 2010;53:1121-6.

16. van de Poll-Franse LV, Mols F, Gundy CM, Creutzberg CL, Nout RA, Verdonck-de Leeuw IM, Taphoorn MJ, Aaronson NK. Normative data for the EORTC QLQ-C30 and EORTC-sexuality items in the general Dutch population. Eur J Cancer 2011;7:667-75.

17. van de Poll-Franse LV, Horevoorts N, van Eenbergen M, Denollet J, Roukema JA, Aaronson NK, Vingerhoets A, Coebergh JW, de Vries J, Essink-Bot ML, Mols F. The Patient Reported Outcomes Following Initial treatment and Long term Evaluation of Survivorship registry: scope, rationale and design of an infrastructure for the study of physical and psychosocial outcomes in cancer survivorship cohorts. Eur J Cancer 2011;47:2188-94. 
18. Martijnse IS, Dudink RL, Kusters M, Rutten HJ, Nieuwenhuijzen GA, Wasowicz-Kemps DK. Patent blue staining as a method to improve lymph node detection in rectal cancer following neoadjuvant treatment. Eur J Surg Oncol 2012;38:252-8.

19. Martijnse IS, Dudink RL, Kusters M, Vermeer TA, West NP, Nieuwenhuijzen GA, van Lijnschoten I, Martijn H, Creemers GJ, Lemmens VE, van de Velde CJ, Sebag-Montefiore D, et al. T3+ and t4 rectal cancer patients seem to benefit from the addition of oxaliplatin to the neoadjuvant chemoradiation regimen. Ann Surg Oncol 2012;19:392-401.

20. Rutten HJ, Mannaerts GH, Martijn H, Wiggers T. Intraoperative radiotherapy for locally recurrent rectal cancer in The Netherlands. Eur J Surg Oncol 2000;26 Suppl A:S16-20.

21. Mannaerts GH, Martijn H, Crommelin MA, Dries W, Repelaer van Driel OJ, Rutten HJ. Feasibility and first results of multimodality treatment, combining EBRT, extensive surgery, and IOERT in locally advanced primary rectal cancer. Int J Radiat Oncol Biol Phys 2000;47:425-33.

22. Aaronson NK, Ahmedzai S, Bergman B, Bullinger M, Cull A, Duez NJ, Filiberti A, Flechtner H, Fleishman SB, de Haes JC, et al. The European Organization for Research and Treatment of Cancer QLQ-C30: a quality-of-life instrument for use in international clinical trials in oncology. J Natl Cancer Inst 1993; 85:365-76.

23. Sprangers MAG, te Velde A, Aaronson NK. The Construction and Testing of the EORTC Colorectal Cancer-specific Quality of Life Questionnaire Module (QLQ-CR38). Eur J Cancer 1999;35:238-47.

24. Thong MS, Mols F, Lemmens VE, Creemers GJ, Slooter GD, van de Poll-Franse LV. Impact of chemotherapy on health status and symptom burden of colon cancer survivors: a population-based study. Eur J Cancer 2011;47:1798-807.

25. Thong MS, Mols F, Lemmens VE, Rutten HJ, Roukema JA, Martijn H, van de Poll-Franse LV. Impact of preoperative radiotherapy on general and disease-specific health status of rectal cancer survivors: a population-based study. Int J Radiat Oncol Biol Phys 2011;81:e49-58.

26. Traa MJ, De Vries J, Roukema JA, Rutten HJT, Den Oudsten BL. The sexual health care needs after colorectal cancer: The view of patients, partners, and health care professionals. Support Care Cancer 2014;22:763-72.

27. Flynn KE, Reese JB, Jeffery DD, Abernethy AP, Lin L, Shelby RA, Porter LS, Dombeck CB, Weinfurt KP. Patient experiences with communication about sex during and after treatment for cancer. Psychooncology 2012;21:594-601.

28. Thong MS, Mols F, Lemmens VE, Rutten HJ, Roukema JA, Martijn H, van de Poll-Franse LV. Impact of preoperative radiotherapy on general and disease-specific health status of rectal cancer survivors: a population-based study. Int J Radiat Oncol Biol Phys 2011;81:e49-58.

29. Marijnen CA, van de Velde CJ, Putter H, van den Brink M, Maas CP, Martijn H, Rutten HJ, Wiggers T, Kranenbarg EK, Leer JW, Stiggelbout AM. Impact of short-term preoperative radiotherapy on healthrelated quality of life and sexual functioning in primary rectal cancer: report of a multicenter randomized trial. J Clin Oncol 2005;23:1847-58.

30. Ferenschild FT, Vermaas M, Nuyttens JJ, Graveland WJ, Marinelli AW, van der Sijp JR, Wiggers T, Verhoef $\mathrm{C}$, Eggermont AM, de Wilt JH. Value of intraoperative radiotherapy in locally advanced rectal cancer. Dis Colon Rectum 2006;49:1257-65.

31. Dubois JB, Bussieres E, Richaud P, Rouanet P, Becouarn Y, Mathoulin-Pelissier S, Saint-Aubert B, Ychou M. Intra-operative radiotherapy of rectal cancer: results of the French multi-institutional randomized study. Radiother Oncol 2011;98:298-30. 
CHAPTER 6 Does extended surgery influence health-related quality of life in patients with rectal cancer?

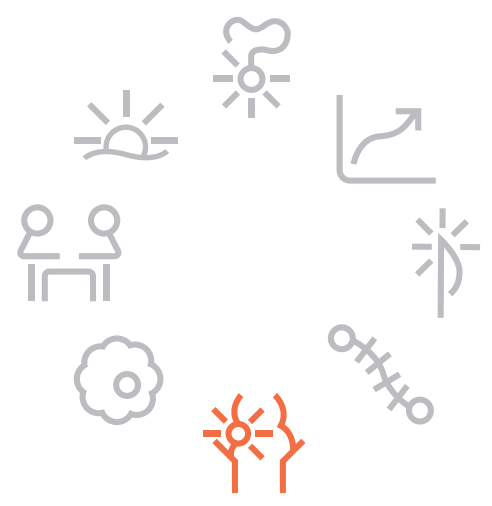

R.G. Orsini

T.A. Vermeer

M.J. Traa

G.A. Nieuwenhuijzen

I.H. de Hingh

H.J. Rutten 


\section{Abstract}

\section{Background}

In locally advanced rectal cancer an extended resection peripheral to the mesorectal fascia is needed in order to achieve a radical resection. The influence of extended resections on health-related quality of life is unclear. Therefore, differences in healthrelated quality of life and sexuality between patients receiving standard and patients receiving extended surgery were examined, with a focus on age.

\section{Methods}

Patients operated on for rectal cancer between 2000 and 2010 were selected from a database and invited to complete the EORTC QLQ-C30 and QLQ-CR38 questionnaires.

All patients were treated in the Catharina Hospital Eindhoven, The Netherlands and received total mesorectal excision surgery or extended surgery for rectal cancer. Health-related quality of life and sexual activity was compared between patients treated with total mesorectal excision surgery and extended surgery and further stratified by age at time of surgery $(<70$ and $\geq 70)$.

\section{Results}

229 (64.1\%) patients with standard surgery and 128 (35.9\%) patients treated with extended resections responded. Extended surgery in patients $<70$ years resulted in lower body image compared to patients $<70$ receiving standard surgery. Patients $\geq 70$ years had lower sexual function and more Male Sexual dysfunction compared to standard surgery patients $<70$ years. In all groups, sexual activity dropped significantly after treatment.

\section{Conclusions}

This study showed no major differences between total mesorectal excision surgery and extended surgery patients except for body image, which was significantly lower in extended surgery patients $<70$ years. In all patient groups treatment for rectal cancer influenced sexual activity dramatically. Awareness of the impact of surgery on healthrelated quality of life and sexuality is needed. 


\section{Introduction}

In rectal tumors confined within the mesorectal fascia, the Total Mesorectal Excision (TME) technique is an anatomical approach with the possibility to preserve the autonomic nerves and the pelvic organs. ${ }^{1}$ However, in locally advanced disease (e.i. tumors invading the mesorectal fascia, or even penetrating into the surrounding pelvic structures) a more extended resection, peripheral to the mesorectal fascia, is needed to avoid positive surgical resection margins. In the pelvis, with the organs packed tightly together, these extended resections may result in damage of the autonomic nerves, other pelvic supporting structures (e.g. sacrum or pelvic floor muscles) or organs (e.g. bladder, genital organs or ureters).

Preoperative treatment with radiotherapy and concomitant chemotherapy can effectively downsize and even downstage locally advanced tumors and reduce the threat of an involved margin and might enable a more preservative approach. ${ }^{2-4}$ However, despite neoadjuvant treatment, extended resections are inevitable in most cases. Due to the increased risk of autonomic pelvic nerve damage and the mutilating effects of these resections, the patients' health-related quality of life (HRQL) and sexual functioning may be affected. One third of the men and almost half of the women have sexual dysfunction before treatment, ${ }^{5}$ in contrast to $20-30 \%$ of males and $40-45 \%$ of women in the general population. ${ }^{6}$ In particular, those patients with impaired preoperative sexual function could be at risk for the development of sexual dysfunction postoperatively.

Studies examining the influence of extended resections on sexuality and HRQL in rectal cancer patients are scarce..$^{7-9}$ In addition, differences in HRQL between different age groups were not analyzed even though there is an increasing awareness that HRQL ${ }^{10}$ and sexual function ${ }^{11}$ may change with age, owing to increasing comorbidities. This study was undertaken to examine the differences in HRQL and sexuality between patients undergoing TME surgery and patients undergoing extended surgery, hereby taking age into account.

\section{Materials and methods}

\section{Patients and procedure}

The Catharina Hospital (Eindhoven, the Netherlands) is a tertiary referral center for patients with locally advanced or locally recurrent rectal cancer. Patients receiving surgery for primary (mostly locally advanced) rectal cancer between 2000 and 2010 were selected. All patients who were alive at the time of the study were invited to 
participate in this study. If patients agreed to participate they completed a set of standardized surveys investigating their HRQOL at home. The local ethics committee approved the study.

\section{Multidisciplinary treatment}

All patients had tumors that were identified by endoscopy and confirmed by biopsy, except for patients who received emergency surgery. In addition, most patients received a preoperative magnetic resonance imaging (MRI) of the pelvis for accurate staging. A pretreatment computerized tomography (CT) of thorax and abdomen was performed to exclude the presence of distant metastases. Patients received neoadjuvant treatment according to the Dutch National Guidelines for rectal cancer. ${ }^{12}$ Patients without a threatened circumferential margin and without pathological lymph nodes on MRI were classified as non-advanced disease (NAD). Most of these patients received short course preoperative radiotherapy with 5x5 Gy, except for T1 tumors who were mostly treated without any neo-adjuvant treatment. Patients with locally advanced rectal cancer (LARC i.e. T3+/T4 with or without pathological lymph nodes) received mostly long course chemoradiation up to $50.4 \mathrm{~Gy}$ in 28 fractions. Different neo-adjuvant treatment schemes of the chemotherapy component were used during the study period. ${ }^{13}$

Before surgery patients with LARC received a pre-operative MRI to evaluate whether or not downstaging had occurred. In almost all cases of LARC, surgery was planned 8-10 weeks after completion of neo-adjuvant treatment. In patients with NAD the period between neoadjuvant treatment and surgery was not planned but based on practical and patient related factors. Patients with NAD or patients with LARC without involvement of surrounding structures, either underwent a low anterior resection (LAR) or an abdominoperineal excision (APE) according to TME principles (TME-group). All patients who were treated with APE were operated on in a supine position, allowing for simultaneous access from both sides in these often bulky tumors. Details of this surgical procedure have been described elsewhere. ${ }^{13}$

Patients with advanced disease, in whom involvement of additional structures was identified on the pre-operative MRI, were treated with en bloc resections of these structures with the primary tumour (i.e. extended resections) and thus extending beyond the TME plane. Extended surgery included posterior- and pelvic exenteration, sacral resections, and TME resections with resection of other structures such as urogenital structures (ES-group). Intra-operative radiotherapy boost (IORT) of 10-15 Gy was applied when a positive resection margin was suspected or confirmed by intraoperative frozen section. Details on this multimodality treatment strategy and influence on outcome have been described before. ${ }^{14,15}$ Patients with rectal cancer who were 
treated with transanal endoscopic microsurgery (TEM) or who had distant metastasis at the time of presentation were excluded from this study.

\section{Questionnaires}

Patients completed the European Organization for Research and Treatment of Cancer (EORTC) general Quality of Life Questionnaire (QLQ-C30) ${ }^{16}$ and the disease specific EORTC - ColoRectal 38 (QLQ-CR38). ${ }^{17}$ Both questionnaires are validated for use within the Dutch population. The QLQ-C30 assesses global health status, functional health, and symptom burden. The QLQ-CR38 assesses colorectal cancer specific functional health and symptom burden. For both questionnaires, the items are ranged on a 4-point scale or 7-point scale. All scales were linearly converted to a 0-100 scale according to standard scoring procedures. For the functioning scales and single items, higher scores indicate better functioning; for the symptom scales and single item, higher scores indicate higher symptom burden.

Patients were asked two additiona, I not validated questions. All patients were asked to what extent they were sexually active preoperatively. This question was answered on a 4-point scale: $1=$ As usual, $2=$ To a lesser extent, $3=$ Barely, 4= Not sexually active. Men were asked if they used aids in order to get an erection. This question was answered on a 4-point scale: $1=$ No, $2=$ Yes, pills (e.g., Viagra), 3= Yes, injections, and $4=$ Yes, mechanical devices. Women were asked if they used aids in order to improve lubrication. This question was answered on a 3-point scale: $1=$ No, $2=$ Yes, medication (e.g., estrogen containing I), 3= Other aids (e.g., lubricants).

\section{Statistical analyses}

Differences in clinical and demographic characteristics between patients treated with standard TME and patients treated with extended surgery were compared using ChiSquare or t-test when appropriate. The EORTC-QLQ-C30 and QLQ-CR38 mean scores were compared with analysis of covariance (ANCOVA's) between patients treated with TME surgery and extended surgery and were further stratified by age. The following 4 groups were made: patients treated with TME $<70$ years (TME $<70$ years), patients treated with TME $\geq 70$ years (TME $\geq 70$ years), patients treated with extended surgery $<70$ years ( $E S<70$ years) and patients treated with extended surgery $\geq 70$ years (ES $\geq 70$ years). Confounding background variables included for adjustment in these analyses were determined a priori and chosen to be sex and time since surgery. The estimated marginal means were examined in order to determine which groups differed significantly from each other (a Bonferroni correction was applied). The analyses were re-performed after excluding patients who had disease recurrence at time of survey to see if these patients have negatively influenced the HRQL scores. 
The scores on the sexuality questions were dichotomized to determine the prevalence of sexual problems. Patients who reported none to minor problems (not at all - a little bit) were categorized as not having sexual problems, while patients who reported more severe problems (quite some - very much) were categorized as having sexual problems. Questions regarding sexual activity were dichotomized into 0 (not sexually active) or 1 (sexually active). Statistical differences were indicated if $\mathrm{p}<0.05$ (two-sided). All analyses were performed using SPSS 19.0.

\section{Results}

\section{Clinical and demographical data}

In total, 357 patients, with a mean follow up of 3.5 years completed the questionnaire, which reflected a response rate of approximately 85\%. A total of 229 (64.1\%) patients were treated according to TME principles; 126 (55.0\%) patients were $<70$ years (TME $<70$ years) and 103 (45.0\%) were $\geq 70$ years (TME $\geq 70$ years). In addition, a total of 128 (35.9\%) patients were treated with extended surgery, of whom 78 (60.9\%) were $<70$ years ( $E S<70$ years) and 50 (39.1\%) were aged $\geq 70$ years ( $E S \geq 70$ years).

In the ES-group more patients had a cT4 stage than in the TME-group $(p=<0.001)$. Patients in the ES-group were more often treated with chemoradiation $(82.8 \%$ vs. $57.7 \%)$ and long course radiotherapy ( $11.7 \%$ vs. $3.9 \%)$ and less often with short course radiotherapy $(3.1 \%$ vs. $34.5 \%, p<0.001$ ) than patients in the TME-group (see Table 6.1 ). In the TME-group $56.4 \%$ of patients were treated with a LAR and $39.7 \%$ with an APE compared to $40.6 \%$ and $56.3 \%$ respectively in the ES-group $(p<0.001)$. More detailed information on the resection in the ES-group is presented in Table 6.2. Intra operative radiotherapy boost was mainly used in the ES-group (78.9\% vs. $34.1 \%, p<0.001)$. In both groups no significant differences were seen regarding local recurrences and distant metastasis at the time of survey. No significant differences were seen in grade I-IV complications, according to the Clavien-Dindo classification, ${ }^{18}$ between both groups. $(p=0.94)$.

\section{Health-related quality of life and sexual functioning}

After adjustment for age and time since diagnosis, there were no significant differences for most of the EORTC C30 subscales between all groups. The analysis of covariance showed significant differences on Physical function and Financial Problems subscales (see Table 6.3). Physical Function was significantly better in the TME $<70$ years group than in the TME $\geq 70$ group $(p=0.01$ ) and ES $\geq 70$ group $(p=0.02)$. Patients $<70$ years undergoing ES experienced significantly more Financial Problems than patients $\geq 70$ years undergoing TME $(p=0.02)$. 
On the EORTC CR38 subscales, we found significant differences regarding Body Image, Sexual Functioning, Male Sexual Functioning and Weight Loss (see Table 6.3). Patients $<70$ years undergoing ES experienced the lowest Body Image in comparison with all other patient groups $(p=0.001)$. Sexual Functioning was higher in the TME $<70$ years group in comparison with both patient groups $\geq 70$ years (both $p=0.001$ ) and was higher in the ES $<70$ group compared to $E S \geq 70$ years group $(p=0.01)$. Men in the TME $<70$ years group reported less Sexual Dysfunction compared with both patient groups $\geq 70$ years (both $p=0.12$ ). Patients in the $E S \geq 70$ years group experienced more Weight Loss than the TME $<70$ years group $(p=0.04)$.

Table 6.1 Clinical and demographic characteristics.

\begin{tabular}{|c|c|c|c|}
\hline & $\begin{array}{c}\text { TME surgery } \\
N=229\end{array}$ & $\begin{array}{c}\text { Extended surgery } \\
\quad N=128\end{array}$ & p-value \\
\hline Age at time of survey (mean \pm SD) & $67.4(10.2)$ & $66.0(10.0)$ & 0.20 \\
\hline \multirow[t]{2}{*}{ Months since surgery (mean \pm SD) } & $42.2(33.3)$ & $40.7(31.2)$ & 0.70 \\
\hline & $\mathrm{N}(\%)$ & $\mathrm{N}(\%)$ & \\
\hline Male & $145(63.3)$ & 60 (46.9) & 0.003 \\
\hline Neoadjuvant treatment & & & $<0.001$ \\
\hline No neoadjuvant treatment & $9(3.9)$ & $3(2.4)$ & \\
\hline Short course radiotherapy (5x5 Gy) & $79(34.5)$ & $4(3.1)$ & \\
\hline Long course radiotherapy & $9(3.9)$ & $15(11.7)$ & \\
\hline Chemoradiaton & $132(57.7)$ & $106(82.8)$ & \\
\hline Type of surgery & & & $<0.001$ \\
\hline Low anterior resection & $129(56.4)$ & $52(40.6)$ & \\
\hline Abdominoperineal excision & $91(39.7)$ & $72(56.3)$ & \\
\hline Hartmann's procedure & $9(3.9)$ & $4(3.1)$ & \\
\hline Intra operative radiotherapy (IORT) & $78(34.1)$ & $101(78.9)$ & $<0.001$ \\
\hline Radical surgery & $220(96.9)$ & $112(88.2)$ & 0.001 \\
\hline Local Recurrence & $4(1.7)$ & $2(1.6)$ & 0.90 \\
\hline Distant Metastases & $19(8.3)$ & $8(6.3)$ & 0.48 \\
\hline Clinical T stage & & & $<0.001$ \\
\hline T1-2 & $41(17.9)$ & $1(0.8)$ & \\
\hline T3 & $119(52.0)$ & $25(19.5)$ & \\
\hline T4 & $66(28.8)$ & $100(78.1)$ & \\
\hline Missing & $3(1.3)$ & $2(1.6)$ & \\
\hline Clinical $\mathrm{N}$ stage & & & 0.08 \\
\hline NO & $120(52.4)$ & $53(41.4)$ & \\
\hline N1 & $56(24.4)$ & $30(23.4)$ & \\
\hline $\mathrm{N} 2$ & $32(14.0)$ & $25(19.6)$ & \\
\hline Missing & $21(9.2)$ & $20(15.6)$ & \\
\hline \multicolumn{4}{|l|}{ Postoperative abdominal complications } \\
\hline Presacral abscess & $22(9.6)$ & $11(8.6)$ & 0.75 \\
\hline Anastomic leakage & $8(3.5)$ & $6(4.7)$ & 0.58 \\
\hline
\end{tabular}

$\mathrm{TME}=$ total mesorectal excision. 
Table 6.2 Details on resection of adjacent organs in patients undergoing extended surgery.

\begin{tabular}{lcc}
\hline & Men & Women \\
& $N=60(\%)$ & \\
\hline Prostate & $18(30)$ & \\
Partial & $7(11,7)$ & \\
Complete & $39(65,0)$ & \\
Vesicles (single or both) & & $22(32,3)$ \\
Vaginal wall & & $10(14,7)$ \\
$\quad$ without reconstruction & & $36(52,9)$ \\
with reconstruction* & & $44(64,7)$ \\
Uterus & & $2(2,9)$ \\
Ovaries (single or both) & & $3(4,4)$ \\
Bladder & & - \\
Partial & $9(15,0)$ & \\
Ureter re-implantation (e.g. psoas hitch) & $4(6,7)$ & $4(5,8)$ \\
Cystectomy with urinary diversion (e.g. Bricker) & $6(10,0)$ & $2(2,9)$ \\
Sacrum & & $6(8,8)$ \\
$\quad$ S3/S4 & $2(3,4)$ & $4(6,7)$ \\
Os coccygis & $3(5,0)$ & \\
Additional bowel resection & & \\
\hline
\end{tabular}

In most patients multiple adjacent organs were removed. *vertical rectus abdominis myocutaneous flap.

For men, no significant differences in preoperative sexual activity between the four treatment groups were observed. Compared with the TME $<70$ years group postoperative sexual activity in males was significantly worse in patients $\geq 70$ years (both TME and ES-groups) and patients $<70$ years in the ES group $(p<0.001)$. In addition, in all groups a decline in sexual activity was seen (Figure 6.1). Male patients in the TME $<70$ years experienced fewer erection problems $(60.0 \%$ vs. $70.6 \%, 78.2 \%$ and $65.4 \%$ respectively $\mathrm{p}=0.04)$ and ejaculation problems $(56.6 \%$ vs. $72.4 \%, 75.6 \%$ and $89.5 \%$ respectively $p=0.02$ ) compared with $E S<70$ years and $E S$ and TME $\geq 70$ years groups. For men, no significant differences were seen in the use of sexual aids.

In women, their preoperative sexual activity was significantly better in the TME $<70$ years group compared with patients in both ES-groups and TME $\geq 70$ years patients $(p=0.03)$. Postoperatively, a decline in sexual activity was also observed in female patients, but no significant difference in sexual activity was seen between the 4 treatment groups (Figure 6.2). In females no significant differences in the experienced lubrication problems, dyspareunia, and the use of aids were observed.

Local disease recurrence or distant metastasis at time of survey did not have a significant effect on the HRQL scores. 
Table 6.3 Mean scores (SD) of general and disease specific health status by type of surgery stratified by age at time of survey ( $<70$ years and $\geq 70$ years).

\begin{tabular}{|c|c|c|c|c|c|}
\hline & \multicolumn{2}{|c|}{$<70$ years } & \multicolumn{2}{|c|}{$\geq 70$ years } & \multirow[b]{2}{*}{$p$-value } \\
\hline & $\begin{array}{c}\text { TME surgery } \\
\qquad N=126\end{array}$ & $\begin{array}{l}\text { Extended surgery } \\
\qquad N=78\end{array}$ & $\begin{array}{c}\text { TME surgery } \\
\qquad N=103\end{array}$ & $\begin{array}{l}\text { Extended surgery } \\
\qquad N=50\end{array}$ & \\
\hline \multicolumn{6}{|l|}{ EORTC C30 subscales } \\
\hline Global quality of life $e^{b}$ & $71.3 \pm 22.6$ & $67.7 \pm 22.0$ & $67.6 \pm 23.1$ & $65.3 \pm 26.3$ & 0.25 \\
\hline Physical function ${ }^{b}$ & $84.7 \pm 17.9$ & $77.4 \pm 19.8$ & $76.5 \pm 21.4$ & $74.7 \pm 22.2$ & 0.003 \\
\hline Role function ${ }^{\mathrm{b}}$ & $77.2 \pm 27.9$ & $74.3 \pm 27.8$ & $74.8 \pm 26.7$ & $72.2 \pm 33.0$ & 0.52 \\
\hline Emotional function ${ }^{\mathrm{b}}$ & $84.7 \pm 16.8$ & $80.4 \pm 23.3$ & $83.8 \pm 21.1$ & $81.9 \pm 21.6$ & 0.66 \\
\hline Cognitive function $^{\mathrm{b}}$ & $85.5 \pm 19.5$ & $80.6 \pm 22.7$ & $83.7 \pm 21.6$ & $85.1 \pm 20.4$ & 0.58 \\
\hline Social function ${ }^{b}$ & $81.0 \pm 23.2$ & $75.6 \pm 26.7$ & $82.7 \pm 24.5$ & $79.9 \pm 25.5$ & 0.33 \\
\hline Fatigue $^{c}$ & $22.9 \pm 22.4$ & $27.9 \pm 23.6$ & $25.6 \pm 23.0$ & $32.2 \pm 27.4$ & 0.10 \\
\hline Nausea / vomiting ${ }^{c}$ & $3.0 \pm 8.5$ & $4.8 \pm 12.6$ & $3.9 \pm 9.1$ & $4.5 \pm 11.8$ & 0.91 \\
\hline Pain $^{c}$ & $16.3 \pm 22.0$ & $18.0 \pm 24.8$ & $13.4 \pm 20.5$ & $15.0 \pm 23.1$ & 0.76 \\
\hline Dyspnoea $^{\mathrm{C}}$ & $13.2 \pm 23.2$ & $13.7 \pm 22.1$ & $15.0 \pm 22.3$ & $18.4 \pm 26.4$ & 0.50 \\
\hline Insomnia $^{c}$ & $16.9 \pm 23.9$ & $17.8 \pm 28.4$ & $20.6 \pm 28.5$ & $20.8 \pm 27.2$ & 0.79 \\
\hline Appetite loss ${ }^{c}$ & $5.7 \pm 15.7$ & $6.8 \pm 18.3$ & $4.6 \pm 12.4$ & $8.2 \pm 21.0$ & 0.66 \\
\hline Constipation $^{c}$ & $7.5 \pm 18.0$ & $9.9 \pm 21.4$ & $7.8 \pm 17.7$ & $11.8 \pm 25.3$ & 0.64 \\
\hline Financial problems ${ }^{c}$ & $9.7 \pm 21.2$ & $16.4 \pm 26.7$ & $6.3 \pm 16.2$ & $8.3 \pm 21.2$ & 0.02 \\
\hline \multicolumn{6}{|l|}{ EORTC CR38 subscales } \\
\hline Body image $^{b}$ & $77.7 \pm 21.4$ & $64.5 \pm 31.4$ & $77.8 \pm 24.4$ & $78.9 \pm 22.5$ & 0.001 \\
\hline Future perspective $^{b}$ & $68.8 \pm 26.1$ & $63.4 \pm 29.7$ & $68.4 \pm 28.8$ & $72.1 \pm 26.7$ & 0.32 \\
\hline Sexual functioning ${ }^{\mathrm{b}}$ & $30.3 \pm 28.6$ & $22.5 \pm 25.2$ & $16.3 \pm 21.8$ & $11.6 \pm 16.9$ & $<0.001$ \\
\hline Sexual enjoyment ${ }^{\mathrm{b}}$ & $62.1 \pm 29.4$ & $48.1 \pm 25.0$ & $61.1 \pm 23.4$ & $44.4 \pm 23.6$ & 0.06 \\
\hline Micturition problems ${ }^{c}$ & $22.7 \pm 15.1$ & $25.2 \pm 16.6$ & $25.9 \pm 16.7$ & $29.5 \pm 18.0$ & 0.08 \\
\hline Chemotherapy side effects $^{c}$ & $11.0 \pm 14.1$ & $15.2 \pm 16.8$ & $13.3 \pm 16.6$ & $18.6 \pm 19.8$ & 0.07 \\
\hline Gastro-intestinal problems ${ }^{c}$ & $20.4 \pm 15.2$ & $21.6 \pm 16.1$ & $17.7 \pm 13.8$ & $19.1 \pm 15.3$ & 0.54 \\
\hline Male sexual dysfunction ${ }^{c}$ & $60.1 \pm 32.2$ & $72.6 \pm 33.2$ & $79.4 \pm 27.8$ & $85.1 \pm 27.2$ & 0.002 \\
\hline Female sexual dysfunction $^{c}$ & $45.2 \pm 33.0$ & $42.2 \pm 30.1$ & $27.4 \pm 34.4$ & $16.7 \pm 24.8$ & 0.16 \\
\hline Stoma-related problems ${ }^{c}$ & $23.6 \pm 16.2$ & $29.4 \pm 21.2$ & $24.6 \pm 21.8$ & $21.2 \pm 14.8$ & 0.31 \\
\hline Weight loss ${ }^{c}$ & $7.3 \pm 16.2$ & $9.6 \pm 19.6$ & $10.6 \pm 21.6$ & $15.6 \pm 28.1$ & 0.04 \\
\hline
\end{tabular}

EORTC= European Organization for Research and Treatment of Cancer; QLQ-C30=general quality of life questionnaire; QLQ-CR38=disease-specific quality-of-life questionnaire ColoRectal 38 ; TME=total mesorectal excision. The EORTC C30 subscale diarrhoea and the EORTC C38 subscale Defaecation Problems mean scores, were not computed because this is not applicable for most patients in the extended surgery group. ${ }^{a} p$-values were adjusted for sex and time since surgery; ${ }^{b}$ Higher scores indicate better functioning; ${ }^{c}$ Higher scores indicate more problems.

\section{Discussion}

Studies focusing on the influence of TME surgery on HRQL report contrary results regarding $L A R$ and $A P E,{ }^{19-24}$ although a meta-analyses reported no significant differences between APR and LAR. ${ }^{25}$ However, studies focusing on the relation between the extend of rectal cancer surgery and HRQL and sexuality are scarce, but available studies could not demonstrate large differences on most of the HRQL subscales. ${ }^{7-9}$ Accordingly, our study showed no major differences in HRQL between patients treated with TME surgery compared to extended surgery, with the exception of Body Image. In 
a previous study, no differences in HRQL between patients with locally advanced disease and patients with non-advanced disease were found accordingly. ${ }^{26}$ All these results indicate that $\mathrm{HRQL}$ after rectal cancer treatment is not directly related to surgery or the type of tumor. The lack of differences in most of the HRQL might be explained by a shift in expectations (e.g. response shift). This may be due to the fact that patients learn to cope with their disabilities because they are a part of their curative treatment.

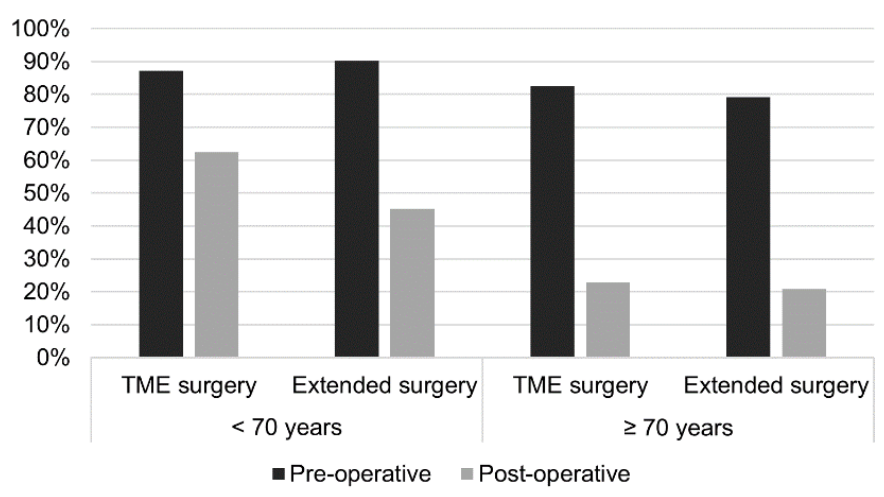

Figure 6.1 Sexual activity pre- and post-operative in men. Post-operative sexual activity in males was significantly lower in patients $\geq 70$ years and patients $<70$ years in the ES-group compared to TME patients $<70$ years $(p<0.001)$.

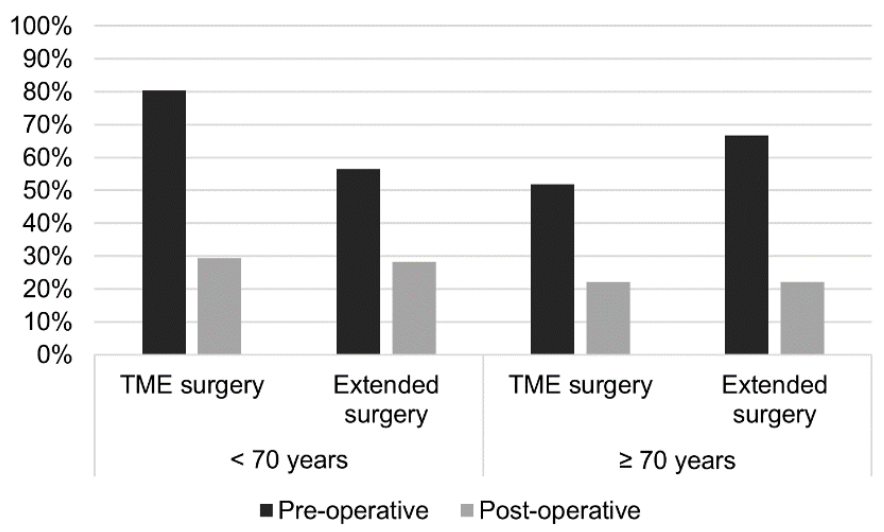

Figure 6.2 Sexual activity pre-and postoperative in women. Pre-operative sexual activity in females was significantly lower in patients $\geq 70$ years and patients $<70$ years in the ES-group $(p<0.03)$. 
However, one aspect of HRQOL that has been previously found to be affected by treatment (e.g. surgery, (neo)adjuvant treatments) and surgery related factors (e.g. a stoma, excessive blood loss, or complications) is the patients' sexual functioning. ${ }^{5,11,27-30}$ Our study showed that the patients $<70$ years undergoing TME surgery had significantly better postoperative sexual function and less male sexual dysfunction compared to patients $\geq 70$ years, regardless of treatment. The biological decline in sexual activity related to aging might play a role here. No significant difference on sexual function and male sexual dysfunction subscale was found between TME surgery and extended surgery in patients $<70$ years, although erectile and ejaculatory problems were less frequent in TME $<70$ years. In all groups a dramatic postoperative decrease in sexual activity in men, particularly in men $\geq 70$ years, was found. These results are not unexpected: the extent of surgery and the natural decline of sexual activity with increasing age may have caused the steepest decline in sexual activity in the ES $>70$ years group. Because patients in the TME $<70$ years group had less invasive surgery at a younger age, it is no surprise that they are the ones who report the fewest sexual problems. In women, a decline in sexual activity was also observed, but no differences regarding post-operative sexual activity and problems were found. The low percentage of postoperative sexual active women (25.6\%), might play a role. However, a high prevalence of sexual dysfunction after treatment is found, which emphasizes the need to asses sexual functioning before and after treatment.

Even though our study showed some significant differences, our study was an observational cross-sectional study. The cross-sectional design does not allow us to make any causal interferences and the observational part of our study implicates that we cannot clearly determine if surgery and age are the sole determining factors, since we could not control for other treatment related differences between the study populations (e.g. type of (neo)adjuvant treatment and IORT). The majority of our patients received neoadjuvant treatment followed by tumor resection and IORT, and therefore do not reflect the standard population.

We expected to find large differences on the sexuality and micturition subscales between TME surgery and extended surgery because of the increased risk of pelvic nerve damage. In males $<70$ years extended resections negatively influenced erectile function and ejaculation. But differences on the EORTC sexuality subscales were not seen. Although large differences could not be found in this studied population, we believe that, on an individual patient level, these differences do exist. Sexual function and $\mathrm{HRQL}$ is not only influenced by treatment related factors but also by other patient related factors like pre-operative sexual activity and psychosocial functioning. HRQL and sexuality are both multifactorial adaptive processes. This makes it difficult to detangle the influence of one specific treatment on HRQL and sexuality, not only in our study, but in colorectal cancer research in general. The detangling of influencing factors 
becomes even more complicated because the multiple treatment strategies used, differ on an international level. This hampers clear comparisons across studies. In addition, especially in an increasingly aging population, future studies should be aware that age and aging plays an important role in HRQL.

In the end, research may help to unravel the biopsychosocial factors associated with HRQL more specifically. In order to do this adequately, it might be beneficial to focus on specific quality of life domains, for example with the use of specific domain related questionnaires or more qualitative studies (e.g. focus groups / interviews) in order to bring the specific differences to light.

The strengths of this study are the large patient population, the availability of clinical data, and the use of validated and reliable questionnaires. However, there are also some limitations that have to be addressed. No information was available on the patients' functioning before treatment, except for the retrospective question on whether or not patients were sexually active preoperatively. Therefore, only associations of an effect of a cancer diagnosis and treatment on HRQL functioning can be found, but causality cannot be accurately assessed. Even though the EORTC QLQCR38 is one of the most used questionnaires to assess sexual functioning, it provides limited information since sexual functioning is assessed with only 5 questions regarding this subject. In our hospital patients receive IORT when an involved surgical margin is suspected. The impact of IORT on HRQOL, especially with regard to sexuality and urogenital items, is not known and future studies are still warranted to examine the additive effects. Finally, we are aware of the heterogeneity of the patients in the extended resection groups, but an analysis based on extent of resection would resulted in too-low-sample sizes to analyze the effects appropriately.

\section{Conclusion}

This study showed no major differences between TME surgery and extended surgery patients in health-related quality of life and sexual function except for Body Image, which was significantly lower in patients younger than 70 years treated with extended surgery. A dramatic drop in sexual function was observed, regardless of surgical treatment. Although this study found no major influence of extended surgery on HRQL, one should be aware of the influence of extended resections in the individual patient. The high prevalence of sexual dysfunction after treatment deserves more attention and patients should be offered adequate counseling in order to manage potential sexual problems. 


\section{References}

1. Heald RJ, Ryall RD. Recurrence and survival after total mesorectal excision for rectal cancer. Lancet 1986;1:1479-82.

2. Bujko K, Nowacki MP, Nasierowska-Guttmejer A, Michalski W, Bebenek M, Kryj M. Long-term results of a randomized trial comparing preoperative short-course radiotherapy with preoperative conventionally fractionated chemoradiation for rectal cancer. Br J Surg 2006;93:1215-23.

3. Bujko K, Kepka L, Michalski W, Nowacki MP. Does rectal cancer shrinkage induced by preoperative radio(chemo)therapy increase the likelihood of anterior resection? A systematic review of randomised trials. Radiother Oncol 2006;80:4-12.

4. Valentini V, van Stiphout RG, Lammering G, et al. Nomograms for predicting local recurrence, distant metastases, and overall survival for patients with locally advanced rectal cancer on the basis of European randomized clinical trials. J Clin Oncol 2011;29:3163-72.

5. Hendren SK, O'Connor BI, Liu M, et al. Prevalence of male and female sexual dysfunction is high following surgery for rectal cancer. Ann Surg 2005;242:212-23.

6. Lewis RW, Fugl-Meyer KS, Bosch R, et al. Epidemiology/risk factors of sexual dysfunction. J Sex Med 2004;1:35-9.

7. Austin KK, Young JM, Solomon MJ. Quality of life of survivors after pelvic exenteration for rectal cancer. Dis Colon Rectum 2010;53:1121-6.

8. Guren MG, Wiig JN, Dueland S, et al. Quality of life in patients with urinary diversion after operation for locally advanced rectal cancer. Eur J Surg Oncol 2001;27:645-51.

9. Palmer G, Martling A, Lagergren P, Cedermark B, Holm T. Quality of life after potentially curative treatment for locally advanced rectal cancer. Ann Surg Oncol 2008;15:3109-17.

10. Vissers PA, Thong MS, Pouwer F, Zanders MM, Coebergh JW, van de Poll-Franse LV. The impact of comorbidity on Health-Related Quality of Life among cancer survivors: analyses of data from the PROFILES registry. J Cancer Surviv 2013;7:602-13.

11. Traa MJ, de VJ, Roukema JA, Den Oudsten BL. Sexual (dys)function and the quality of sexual life in patients with colorectal cancer: a systematic review. Ann Oncol 2012;23:19-27.

12. Dutch National Guidelines Rectal Cancer. http://www.oncoline.nl/rectalcancer. 2013.

13. Martijnse IS, Dudink RL, West NP, et al. Focus on extralevator perineal dissection in supine position for low rectal cancer has led to better quality of surgery and oncologic outcome. Ann Surg Oncol 2012; 19:786-93.

14. Dresen RC, Gosens MJ, Martijn H, et al. Radical resection after IORT-containing multimodality treatment is the most important determinant for qutcome in patients treated for locally recurrent rectal cancer. Ann Surg Oncol 2008;15:1937-47.

15. Mannaerts GH, Martijn H, Crommelin MA, Dries W, Repelaer van Driel OJ, Rutten HJ. Feasibility and First results of multimodality treatment, combining EBRT, extensive surgery, and IOERT in locally advanced primary rectal cancer. Int J Radiat Oncol Biol Phys 2000;47:425-33.

16. Aaronson NK, Ahmedzai S, Bergman B, et al. The European organization for research and treatment of cancer QLQ-C30: a quality-of-life instrument for use in international clinical trials in oncology. $J \mathrm{Natl}$ Cancer Inst 1993;85:365-76.

17. Sprangers MA, te VA, Aaronson NK. The construction and testing of the EORTC colorectal cancerspecific quality of life questionnaire module (QLQ-CR38). European Organization for Research and Treatment of Cancer Study Group on Quality of Life. Eur J Cancer 1999;35:238-47.

18. Clavien PA, Barkun J, de Oliveira ML, et al. The clavien-dindo classification of surgical complications: five-year experience. Ann Surg 2009;250:187-96.

19. Grumann MM, Noack EM, Hoffmann IA, Schlag PM. Comparison of quality of life in patients undergoing abdominoperineal extirpation or anterior resection for rectal cancer. Ann Surg 2001;233: 149-56.

20. Engel J, Kerr J, Schlesinger-Raab A, Eckel R, Sauer H, Holzel D. Quality of life in rectal cancer patients: a four-year prospective study. Ann Surg 2003;238:203-13.

21. Gosselink MP, Busschbach JJ, Dijkhuis CM, Stassen LP, Hop WC, Schouten WR. Quality of life after total mesorectal excision for rectal cancer. Colorectal Dis 2006;8:15-22. 
22. Kasparek MS, Hassan I, Cima RR, Larson DR, Gullerud RE, Wolff BG. Quality of life after coloanal anastomosis and abdominoperineal resection for distal rectal cancers: sphincter preservation vs quality of life. Colorectal Dis 2011;13:872-7.

23. Guren MG, Eriksen MT, Wiig JN, et al. Quality of life and functional outcome following anterior or abdominoperineal resection for rectal cancer. Eur J Surg Oncol 2005;31:735-42.

24. How P, Stelzner S, Branagan G, et al. Comparative quality of life in patients following abdominoperineal excision and low anterior resection for low rectal cancer. Dis Colon Rectum 2012;55:400-6.

25. Cornish JA, Tilney HS, Heriot AG, Lavery IC, Fazio VW, Tekkis PP. A meta-analysis of quality of life for abdominoperineal excision of rectum versus anterior resection for rectal cancer. Ann Surg Oncol 2007; 14:2056-68.

26. Traa MJ, Orsini RG, Oudsten BL, et al. Measuring the health-related quality of Life and sexual functioning of patients with rectal cancer: does type of treatment matter? Int J Cancer 2014;134: 979-87.

27. Lange $M M$, Marijnen $C A$, Maas $C P$, et al. Risk factors for sexual dysfunction after rectal cancer treatment. Eur J Cancer 2009;45:1578-88.

28. Schmidt CE, Bestmann B, Kuchler T, Kremer B. Factors influencing sexual function in patients with rectal cancer. Int J Impot Res 2005;17:231-8.

29. Thong MS, Mols F, Lemmens VE, et al. Impact of preoperative radiotherapy on general and diseasespecific health status of rectal cancer survivors: a population-based study. Int J Radiat Oncol Biol Phys 2011;81:e49-58.

30. Orsini RG, Thong MS, van de Poll-Franse LV, et al. Quality of life of older rectal cancer patients is not impaired by a permanent stoma. Eur J Surg Oncol 2013;39:164-70. 


\section{CHAPTER 7 Quality of life of older rectal}

cancer patients is not impaired by a permanent stoma

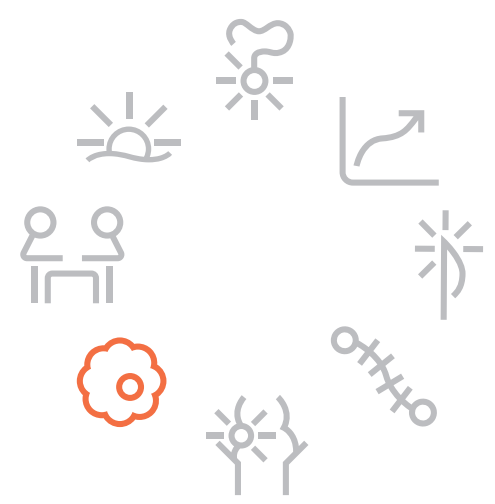

R.G. Orsini

M.S. Thong

L.V. van de Poll-Franse

G.D. Slooter

G.A. Nieuwenhuijzen

H.J. Rutten

I.H. de Hingh 


\section{Abstract}

\section{Background}

The current study was undertaken to investigate the impact of a stoma on the HRQL with a special focus on age.

\section{Methods}

Using the Eindhoven Cancer Registry, rectal cancer patients diagnosed between 19982007 in 4 hospitals were identified. All patients underwent TME surgery. Survivors were approached to complete the SF-36 and EORTC QLQ-C38 questionnaires. HRQL scores of the four groups, stratified by stoma status (stoma / no stoma) and age at operation ( $<70$ and $\geq 70$ years), were compared. The SF-36 and the QLQ-CR38 sexuality subscale scores of the survivors were compared with an age- and sex-matched Dutch norm population.

\section{Results}

Median follow-up of 143 patients was 3.4 years. Elderly had significantly worse physical function $(p=0.0003)$ compared to younger patients. Elderly $(p=0.005)$ and patients without a stoma $(p=0.009)$ had worse sexual functioning compared to younger patients and patients with a stoma. Older males showed more sexual dysfunction $(p=0.01)$ when compared to younger males. In comparison with the normative population, elderly with a stoma had worse physical function $(p<0.01)$, but slightly better mental health $(p<0.05)$. Elderly without a stoma had better emotional role function $(p<0.01)$, and younger patients had worse sexual functioning and enjoyment (both $p<0.0001$ ).

\section{Conclusions}

Older patients with a stoma have comparable HRQL to older patients without a stoma or the normative population, indicating the feasibility of a permanent stoma for elderly patients with a low situated rectal carcinoma. The negative impact of treatment on sexual functioning as found in the current study calls for further attention to alleviate this problem in sexually active patients. 


\section{Introduction}

In rectal cancer surgery patients typically undergo a sphincter preserving procedure (low anterior resection: LAR) or abdominoperineal resection (APR) resulting in a permanent colostomy. The choice for one of these procedures depends on the level of the tumour, the technical feasibility to perform an anastomosis and the condition of the patient. Usually a LAR is preferred when a 1-2 cm tumor-free distal resection margin is feasible. ${ }^{1,2}$ However, the number of postoperative problems after LAR is high, especially after neo-adjuvant radiochemotherapy with anastomotic leakage being the most feared complication due to its potentially devastating consequences. ${ }^{3}$ Besides these traditional clinical arguments, health-related quality of life (HRQL) is increasingly accepted as an indicator for treatment efficacy. ${ }^{4,5}$ Intuitively, it is conceivable that avoiding a permanent stoma will result in a better HRQL. However, this was challenged by two recent reviews investigating the influence of a stoma on the HRQL showing no relevant impact of a permanent stoma. ${ }^{4,5}$

Balancing the assumed benefits of a colo-anal anastomosis against the potential postoperative complications may be especially difficult in the elderly and frail patients. Patients with comorbidity and less physiologic reserves may not be capable to cope with complications. An alternative for these patients could be a Hartmann's procedure with resection of the tumor but without restoration of bowel continuity.

Knowledge of the impact of a stoma on the HRQL may help to determine a treatment strategy for elderly patients suffering from rectal cancer that is both safe and preserves a good HRQL. Unfortunately, studies investigating the impact of treatment on the HRQL in elderly rectal cancer patients are scarce. ${ }^{6,7}$ The current study was undertaken to investigate the impact of a stoma on the HRQL with a special focus on age.

\section{Methods}

\section{Setting and participants}

This study is part of a (long-term) cancer survivorship study of rectal cancer patients registered at the Eindhoven Cancer Registry (ECR) which collects data on all newly diagnosed cancer patients in the southern part of the Netherlands. ${ }^{8}$ Patients diagnosed with rectal cancer in the period 1998-2007 were eligible. Details of the selection process have been reported elsewhere. ${ }^{9}$ The survivorship study was designed to evaluate various patient-reported outcomes such as late/long-term effects, and physical and mental health status. Patient-reported outcome data was collected via the PROFILES (Patient Reported Outcomes Following Initial treatment and Long term 
Evaluation of Survivorship) registry. ${ }^{10}$ Data from the PROFILES registry will be available for non-commercial scientific research, subject to study question, privacy and confidentiality restrictions, and registration (www.profilesregistry.nl).

For the current study, all rectal cancer patients (tumor $\leq 10 \mathrm{~cm}$ anal verge) with a completed questionnaire from 4 hospitals were selected; Catharina Hospital (Eindhoven), Elkerliek Hospital (Helmond-Deurne), Maxima Medical Center (EindhovenVeldhoven) and St. Anna Hospital (Geldrop), all situated in the southeast of the Netherlands. Of the 156 eligible patients, only those who underwent TME-surgery (APR or LAR) were selected. Thirteen patients were excluded for the following reasons: transanal endoscopic microsurgery $(n=5)$, distant metastasis at time of surgery $(n=1)$, received radiotherapy only $(n=1)$, lost to follow-up $(n=6)$ resulting in 143 patients.

\section{Data collection}

Eligible survivors received a letter from their treating physician explaining the purpose of the study. The letter explained that by completing and returning the enclosed questionnaire survivors consented to participate in the study and agreed to the linkage of the questionnaire data with their disease history in the ECR. Survivors were reassured that non-participation had no consequences on their follow-up care or treatment. Non-respondents were sent a reminder letter and a questionnaire after 2 months. For this study, routinely collected data on tumor and patient characteristics by the ECR was augmented by extra clinical data extracted by one of the authors (RGO) from the patients' medical records. Extra clinical parameters extracted included distance of the tumor from the anal verge, (neo-)adjuvant treatment, surgical procedure performed, stoma characteristics, postoperative complications, tumor classification and follow-up data on recurrence (local/regional) and metastasis.

\section{Measures}

General HRQL was assessed with the validated Dutch version of the Short Form-36 (SF-36) questionnaire. ${ }^{11}$ The eight subscales include physical functioning (assesses limitations to daily activities such as climbing stairs or lifting groceries), role limitations due to physical health (assesses limitations in work/activities due to physical health), bodily pain, general health perceptions, vitality (assesses energy and fatigue), social functioning, role limitations due to emotional health (assesses limitations in work/activities due to mental health), and mental health (assesses anxiety and depression). All scales were linearly converted to a 0-100 scale according to standard scoring procedures, with higher scores indicating better HRQL.

Disease-specific issues were assessed with the Dutch validated European Organization for Research and Treatment of Cancer (EORTC) module Quality of Life Questionnaire Colorectal 38 (QLQ-CR38). ${ }^{12}$ The QLQ-CR38 assess both functioning and symptom 
burden. Functioning consists of two scales (body image and sexual functioning), two single items (future perspective and sexual enjoyment), seven symptom scales (micturition problems, defecation problems, gastrointestinal symptoms, stoma-related problems, chemotherapy side effects, male and female sexual problems) and an item on weight loss. The items are ranged on a 4-point scale ranging from 1 (not at all) to 4 (very much). All scales were linearly converted to a 0-100 scale according to standard scoring procedures. ${ }^{12}$ For the functioning scales and single items, higher scores indicate better functioning; for the symptom scales and single item, higher scores indicate higher symptom burden.

Self-reported comorbidity was categorized according to an adapted Self-administered Comorbidity Questionnaire (SCQ). ${ }^{13}$ The SCQ also assesses the patient's perceived severity and burden of the comorbid condition. Socioeconomic status was determined by an indicator developed by Statistics Netherlands based on individual fiscal data from the year $\mathbf{2 0 0 0}$ on the economic value of the home and household income, and provided as aggregate level for each postal code (average 17 households), which were then categorized into tertiles. ${ }^{14}$ Body mass index (BMI), marital status, educational level, employment status and smoking were also assessed.

Normative data from the Dutch SF-36 validation study were used to compare the mean subscale scores between the treatment groups and the norm population. ${ }^{11}$

In 2009, CentERdata a research institute at Tilburg University, was assigned to collect normative data on sexuality via the CentERpanel. ${ }^{15}$ The CentERpanel is an online household panel consisting of over 2000 households which are representative of the Dutch-speaking population in the Netherlands. For households without internet access, additional provisions were provided to assist in data collection. In total, 1613 (75\%) cancer-free panel members of $\geq 18$ years completed three items on sexuality from the EORTC-QLQ-CR38. Members were asked to what extent over the past 4 weeks were they: 1 ) interested in sex; 2 ) sexually active; and for those who were sexually active, 3) to what extent was sex enjoyable for them. These three items were scored according to standard EORTC-QLQ-CR38 procedures. ${ }^{12}$ Furthermore, sociodemographic data such as age, sex, marital status, and comorbidity were collected.

\section{Statistical analyses}

All statistical analyses were performed using SPSS (version 17.0 for windows. SPSS Inc., Chicago, IL). Differences in clinical and demographic parameters between groups were compared using chi-square or t-test when appropriate. If normality and homogeneity assumptions were violated, non-parametric tests were used.

Comparisons between the treatment groups (stoma vs. non-stoma) on the SF-36 and QLQ-CR38 mean scores were performed using analysis of covariance (ANCOVA). Both groups were further stratified by age at time of surgery ( $<70$ and $\geq 70$ years). 
Confounding variables were determined a priori. ${ }^{16}$ Variables included for adjustment were comorbidity, level of tumor from anal verge, tumor stage, post-operative complications and disease progression. The mean SF-36 scores of the patient samples were compared with an age and sex-matched Dutch normal population. For this analysis, the groups (norm, stoma and no stoma) were stratified by age ( $<70$ years and $\geq 70$ years) at time of survey. Comparisons were made using the independent sample $t-$ test. Comparisons on the sexuality items of the QLQ-CR-38 between the treatment groups and the Dutch norm population were performed using ANCOVA, adjusted for age, marital status, comorbidity, sex, sex*group. Statistical differences were indicated if a $p$-value $<0.05$ and reported $p$-values were two-sided.

\section{Results}

\section{Clinical and demographic data}

In total, 143 patients were included in this analysis. The median follow-up was 3.4 years (0.8-11.1 years). There were no statistically significant differences in demographic characteristics between patients with a stoma $(n=67)$ and without a stoma $(n=76)$. Marital status and educational level not shown. As may be expected, stoma patients were more likely to have a tumour closer to the anal verge $(p<0.0001)$ and were often treated with an APR $(\mathrm{p}<0.0001)$ (Table 7.1).

\section{HRQL}

After adjustment, there was no significant effect of the presence of a stoma on any of the SF-36 subscales. However, there was a significant age effect $(p=0.0003)$ on physical functioning, with older patients having a worse physical function as compared to younger patients regardless of the presence of a stoma (54.2 \pm 27.5 and $63.2 \pm 27.3$ vs. $76.2 \pm 22.6$ and $78.2 \pm 21.5$, respectively) (Table 7.2).

Comparing the HRQL scores between the 4 groups on the QLQ-CR38 domains revealed significant differences in sexual functioning and male sexual dysfunction. There was a significant age effect with older patients having worse sexual function compared to younger patients $(p=0.005)$. Interestingly, older patients with a stoma had better sexual function as compared to patients without a stoma $(p=0.009)$. In younger patients, sexual functioning was not influenced by the presence of a stoma. Male sexual dysfunction was significantly worse for older patients $(p=0.03)$ but again this was not affected by the presence of a stoma. No significant interaction (stoma status $\mathrm{x}$ age) effect on any the SF-36 and QLQ-C38 items was revealed. 
Table 7.1 Clinical and demographic characteristics of CRC survivors by stoma status at time of survey.

\begin{tabular}{|c|c|c|c|}
\hline & $\begin{array}{l}\text { Stoma } \\
(n=67)\end{array}$ & $\begin{array}{c}\text { Non-stoma } \\
(n=76)\end{array}$ & p-value \\
\hline Age at time of surgery (median \pm SD) & $64.7(11.1)$ & $64.7(9.3)$ & 0.9 \\
\hline \multirow[t]{2}{*}{ Years since initial diagnosis (median \pm SD) } & $36.7(34.1)$ & $42.8(29.8)$ & 0.9 \\
\hline & $N(\%)$ & $N(\%)$ & \\
\hline Male & $41(61.2)$ & $48(63.2)$ & 0.8 \\
\hline Distance from anal verge (median $\pm S D$ ) & $4,0(2.7)$ & $8,2(2.0)$ & $<0.0001$ \\
\hline \multicolumn{4}{|l|}{ Type of surgery } \\
\hline Abdominal perineal resection & $56(83.6)$ & NA & \\
\hline Low anterior resection & $11(16.4)$ & $76(100)$ & $<0.0001$ \\
\hline Intra operative radiotherapy (IORT) & $6(9.0)$ & $3(3.9)$ & 0.2 \\
\hline \multicolumn{4}{|l|}{ Surgical complications ${ }^{\mathrm{a}}$} \\
\hline None & $37(55.2)$ & $54(71.1)$ & \\
\hline Grade I-II & $20(29.9)$ & $13(17.1)$ & \\
\hline Grade IIla & - & $2(2.6)$ & \\
\hline Grade IIIb & $10(14.9)$ & $7(9.2)$ & 0.7 \\
\hline \multicolumn{4}{|l|}{ Neo-adjuvant treatment } \\
\hline No neoadjuvant treatment & $6(9.0)$ & $9(11.8)$ & \\
\hline Short course radiotherapy (5x5 Gy) & $50(74.6)$ & $59(77.6)$ & \\
\hline Long course radiotherapy & $1(1.5)$ & - & \\
\hline Chemoradiation & $10(14.9)$ & $8(10.5)$ & 0.2 \\
\hline Adjuvant chemotherapy & $14(20.9)$ & $8(10.5)$ & 0.1 \\
\hline \multicolumn{4}{|l|}{ pTNM stage } \\
\hline 1 & $37(55.2)$ & $31(40.8)$ & \\
\hline$\| \mathrm{A}$ & $12(17.9)$ & $22(28.9)$ & \\
\hline IIB & $1(1.5)$ & - & \\
\hline IIIA & $4(6.0)$ & $6(7.9)$ & \\
\hline IIIB & $12(17.9)$ & $13(17.1)$ & \\
\hline IIIC & $1(1.5)$ & $4(5.3)$ & 0.2 \\
\hline Local recurrence & - & $1(1.3)$ & 0.3 \\
\hline Distant metastasis & $3(4.5)$ & $9(11.8)$ & 0.1 \\
\hline \multicolumn{4}{|l|}{ Comorbidity } \\
\hline None & $19(28.4)$ & $27(35.5)$ & \\
\hline 1 & $15(22.4)$ & $20(26.3)$ & \\
\hline$>1$ & $33(49.3)$ & $29(38.2)$ & 0.4 \\
\hline
\end{tabular}

Some variables do not add up to $100 \%$ due to missing data. ${ }^{a}$ According to the Clavien-Dindo classification. ${ }^{17}$ grade $1=$ any deviation from normal postoperative course without pharmacological treatment, grade $2=$ complications requiring pharmacotherapeutic intervention, grade $3 a$ complications needing reintervention without general anesthesia, grade $3 \mathrm{~b}$ complication requiring reintervention under general anesthesia of life threatening complication requiring ICU admission.

\section{HRQL compared with normative population}

The SF-36 subscale scores from the 2 groups (stoma vs. no stoma) were compared with a Dutch normative population, stratified by age at time of survey (Figures 7.1 and 7.2). Among the $<70$ years respondents, no significant difference was found in any of the items of the SF-36 (Figure 7.1). Respondents aged $\geq 70$ years with a stoma had a significant worse physical function as compared to the normative population (59.5 vs. 
$74.7 \mathrm{p}<0.01$ ) but a slightly better mental health (80.8 vs. $75.6 \mathrm{p}<0.05$ ) (Figure 7.2). Older patients without a stoma scored better for emotional role function in comparison with the normative population (91 vs. $78.1 \mathrm{p}<0.01$ ). Other items of the SF-36 showed no differences with the normative population.

Table 7.2 Mean scores $( \pm$ SD) of general and disease specific health status of rectal cancer survivors by stoma status stratified by age at time of surgery ( $<70$ and $\geq 70$ years).

\begin{tabular}{|c|c|c|c|c|c|c|}
\hline & \multicolumn{2}{|c|}{ Stoma } & \multicolumn{2}{|c|}{ no stoma } & \multirow{2}{*}{\multicolumn{2}{|c|}{ Ancova* $^{*}$}} \\
\hline & \multirow{2}{*}{$\begin{array}{c}<70 \text { years } \\
(n=44)\end{array}$} & \multirow{2}{*}{$\begin{array}{c}\geq 70 \text { years } \\
(n=23)\end{array}$} & \multirow{2}{*}{$\begin{array}{c}<70 \text { years } \\
(n=57)\end{array}$} & \multirow{2}{*}{$\begin{array}{c}\geq 70 \text { years } \\
(n=19)\end{array}$} & & \\
\hline & & & & & Stoma effect & Age effect \\
\hline \multicolumn{7}{|l|}{ SF-36 } \\
\hline General health & $66.6 \pm 22.0$ & $61.2 \pm 21.6$ & $64.2 \pm 22.4$ & $54.2 \pm 16.5$ & n.s. & n.s. \\
\hline Physical function & $76.2 \pm 22.6$ & $54.2 \pm 27.5$ & $78.2 \pm 21.5$ & $63.2 \pm 27.3$ & n.s. & 0.0003 \\
\hline Role function - physical & $65.3 \pm 40.3$ & $51.8 \pm 43.9$ & $71.5 \pm 43.0$ & $62.5 \pm 43.9$ & n.s. & n.s. \\
\hline Bodily pain & $77.3 \pm 23.5$ & $77.3 \pm 26.2$ & $76.4 \pm 25.6$ & $78.9 \pm 24.0$ & n.s. & n.s. \\
\hline Vitality & $67.4 \pm 17.5$ & $65.0 \pm 18.8$ & $67.8 \pm 21.1$ & $64.4 \pm 18.8$ & n.s. & n.s. \\
\hline Social function & $82.1 \pm 17.7$ & $82.6 \pm 19.8$ & $80.9 \pm 20.5$ & $79.9 \pm 17.2$ & n.s. & n.s. \\
\hline Role function - emotional & $77.0 \pm 37.9$ & $71.9 \pm 37.3$ & $82.3 \pm 32.7$ & $88.9 \pm 19.8$ & n.s. & n.s. \\
\hline Mental health & $78.3 \pm 14.1$ & $79.8 \pm 14.7$ & $76.8 \pm 18.0$ & $74.0 \pm 15.9$ & n.s. & n.s. \\
\hline \multicolumn{7}{|l|}{ EORTC-QLQ-CR38 ${ }^{\mathrm{a}}$} \\
\hline Body image & $68.2 \pm 29.0$ & $67.6 \pm 29.9$ & $76.4 \pm 25.7$ & $84.7 \pm 14.5$ & n.s. & n.s. \\
\hline Future perspective & $65.1 \pm 21.5$ & $73.9 \pm 28.3$ & $70.9 \pm 25.7$ & $70.8 \pm 23.9$ & n.s. & n.s. \\
\hline Sexual function & $25.2 \pm 23.6$ & $17.5 \pm 21.8$ & $24.5 \pm 24.1$ & $12.2 \pm 16.0$ & 0.009 & 0.005 \\
\hline Sexual enjoyment ${ }^{b}$ & $57.3 \pm 24.7$ & $23.8 \pm 25.2$ & $50.0 \pm 35.7$ & 33.3 & NA & n.s. \\
\hline Micturition problems & $28.2 \pm 21.7$ & $29.0 \pm 16.7$ & $26.2 \pm 17.4$ & $33.3 \pm 19.0$ & n.s. & n.s. \\
\hline Chemo & $10.1 \pm 12.4$ & $9.7 \pm 14.7$ & $12.5 \pm 16.0$ & $13.3 \pm 13.4$ & n.s. & n.s. \\
\hline Gastrointestinal problems & $19.0 \pm 15.6$ & $18.4 \pm 13.1$ & $25.2 \pm 16.1$ & $20.8 \pm 11.6$ & n.s. & n.s. \\
\hline Male sexual dysfunction ${ }^{c}$ & $57.6 \pm 37.0$ & $80.5 \pm 26.4$ & $48.5 \pm 34.9$ & $66.7 \pm 39.1$ & n.s. & 0.03 \\
\hline Female sexual dysfunction $^{d}$ & $44.4 \pm 30.0$ & - & $24.3 \pm 33.1$ & - & & \\
\hline Defecation problems & - & - & $25.6 \pm 16.8$ & $28.2 \pm 13.0$ & NA & n.s. \\
\hline Stoma-related problems & $26.7 \pm 17.8$ & $27.0 \pm 26.7$ & - & - & NA & n.s. \\
\hline Weight loss & $3.9 \pm 10.8$ & $4.5 \pm 15.6$ & $9.1 \pm 19.7$ & $6.2 \pm 18.0$ & n.s. & n.s. \\
\hline
\end{tabular}

SF-36 = Short Form 36 Questionnaire; EORTC-QLQ-CR38 = European Organization for Research and Treatment of Cancer (EORTC) module Quality of life Questionnaire -Colorectal 38; n.s. = not significant; NA= not applicable; blank indicates that no statistical analyses could be performed due to missing data ${ }^{a} E O R T C-Q L Q-$ CR38: Body image, future perspective, sexual function and sexual enjoyment scales: higher scores indicate better function; for the other symptom scales: higher scores indicates higher symptom burden. ${ }^{b}$ only one patient $\geq 70$ with no stoma completed the sexual enjoyment item. ${ }^{c}$ due to small numbers per cell, only age at treatment and stoma status were included in the model. ${ }^{d}$ no female $\geq 70$ years provided information about female sexual dysfunction. ${ }^{*}$ p-values were adjusted for confounding variables: level tumor from anal verge (continuous variable), co morbidity, tumor stage (I=T1,T2 without lymph node positivity or metastasis; II=T3T4 without lymph node positivity or metastasis; III any T with lymph node positivity) post-operative complications and disease progression. 


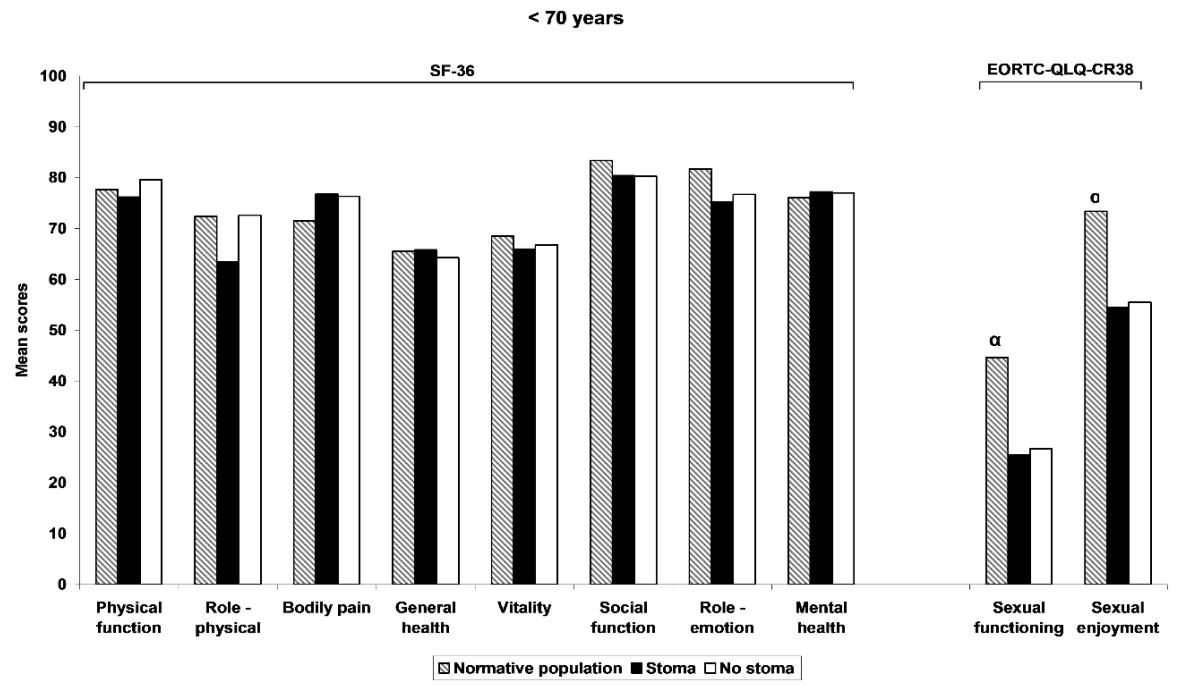

Figure 7.1 Mean SF-36 and EORTC-QLQ-CR38 (sexual function and sexual enjoyment) subscale scores of patients $<70$ years at time of survey are shown according to stoma status in comparison with an age matched Dutch normal population. For the SF-36 subscales, no differences were found between the treatment groups and the age- and sex-matched normative population. Comparisons were made using the independent sample t-tests. $(\alpha)$ For the EORTC-QLQ-CR38 subscales, a significant interaction (ANCOVA, adjusted for age, marital status, comorbidity, sex, sex*group) was found for stoma versus normative population $(p<0.0001)$.

\section{Sexual functioning compared with normative population}

On the sexuality items of the QLQ-CR38, after adjustment for several variables, sexual functioning was significantly better in the normative population as compared to younger rectal cancer patients both with and without a stoma (44.6 vs. 26.7 and 25.5 , respectively, $p<0.0001)$. Similar differences were revealed on sexual enjoyment $(73.4$ vs. 55.5 and 54.5 , respectively, $p<0.0001$ ) (Figure 7.1). Older patients scored slightly poorer on sexual function and sexual enjoyment as compared to the normative population although these differences were not statistically significant (Figure 7.2). 


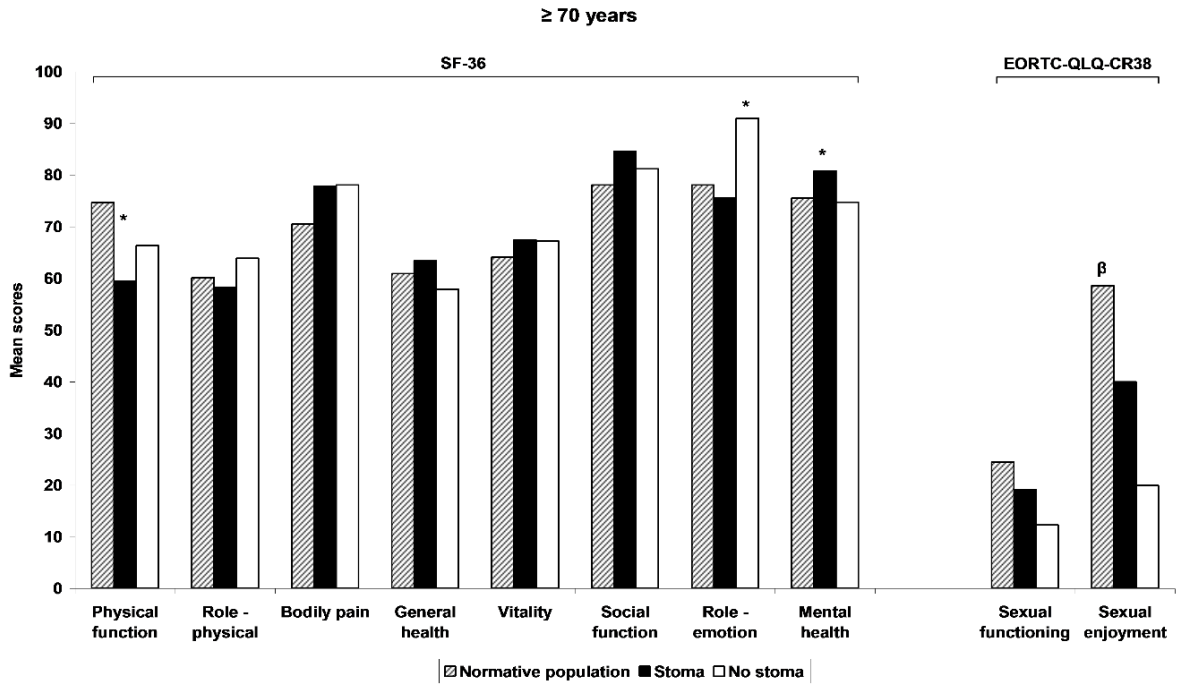

Figure 7.2 Mean SF-36 and EORTC-QLQ-CR38 (sexual function and sexual enjoyment) subscale scores of patients $\geq 70$ years at time of survey are shown according to stoma status in comparison with an age matched Dutch normal population. For the SF-36, an asterisk above the age- and sexmatched normative population indicates a difference (with same significance level) in mean score with survivors, as indicated from independent sample t-tests. When a subgroup of patients has a difference in the mean score to the normative population, then an asterisk was placed above that subgroup. For the EORTC-QLQ-CR38 subscales no significant interaction (ANCOVA, adjusted for age, marital status, comorbidity, sex, sex*group) was found between the treatment groups and the age-and sex-matched normative population. $(\beta)$ Could not be adjusted as the numbers in the clinical groups are too small.

\section{Discussion}

The decision between a LAR with colo-anal anastomosis and LAR/APR with a permanent stoma in a patient with a distal rectal tumor may be difficult. This is especially true for elderly patients where the assumed benefits of the avoidance of a stoma should be outweighed against the potentially life threatening postoperative complications such as anastomotic leakage. When anastomotic leakage occurs in the elderly, the ensuing mortality rate could be up to $57 \%$ in the first 6 months post-operation. Furthermore, other post-operative complications such as abscesses, sepsis and cardiac and pulmonary problems have also been related to a significant increased mortality rate in elderly compared to younger patients. ${ }^{18}$ 


\section{Influence of a stoma on HRQL}

In the current study it was revealed that the presence of a stoma had only a minor influence on the HRQL, irrespective of age. Age itself seemed to be of more impact since older patients experienced worse physical functioning as compared to younger patients irrespective of the presence of a stoma. These findings are in accordance with recently published data, showing that the HRQL levels of older patients do not reach baseline levels even after 2 years post-surgery, which suggests that the elderly will suffer a more permanent impaired physical function after surgery for rectal cancer. ${ }^{7}$ In the QLQ-CR38 sexuality domains, age was related to more dysfunction, irrespective of the presence of a stoma. Interestingly, older patients with a stoma scored significantly better on the sexuality subscales than those without a stoma.

\section{HRQL of rectal cancer patients after treatment}

In order to relate the HRQL of rectal cancer patients after treatment, a comparison was made to a Dutch normative population. This revealed that younger patients have a similar general HRQL as compared to the normative population. Older patients with a stoma had a significant lower physical function but better mental health compared to the normative population. Moreover, older patients without a stoma had a higher emotional role function compared to the normative population. Altogether, HRQL in rectal cancer patients is almost similar to that of a normative population in spite of the extensive treatment that these patients often have undergone. This finding may be somewhat counter intuitive at first but was also demonstrated in other recent studies. ${ }^{19,20}$ This phenomenon is now referred to as "response shift", whereby patients change their internal standards as an adaptation to limitations caused by the disease or its treatment. ${ }^{21}$

\section{Sexual dysfunction following treatment}

In this study the prevalence of sexual dysfunction following treatment is high, particularly when compared to the normative population. Sexual problems are wellknown after rectal cancer as recently reviewed, with an incidence of dysfunction of 23-69\% in men and $30-40 \%$ of previously sexually active patients reporting inactivity following treatment. ${ }^{22}$ Given the high incidence of sexual problems and the impact on the HRQL, this should be part of information provision prior to surgery. Furthermore this problem should be addressed and treated whenever possible in rectal cancer survivors by providing psychosocial and clinical support.

Altogether, the current study shows that the impact of a stoma on the HRQL of rectal cancer patients is small regardless of age. Similar findings were recently reported in a large meta-analysis and Cochrane review. ${ }^{5}$ In addition, other recent studies showed that other specific parameters, such as gender and post-operative complications, have 
more impact on the quality of life than having a stoma. ${ }^{23-25}$ We believe that a permanent stoma is feasible for elderly and frail patients with a low situated rectal tumor, particularly when the patient is at 'high' risk for post-operative complications due to co-morbidities or frail condition.

The strengths of the present study include the availability of clinical data, the usage of a population based sample data, availability of a validated questionnaire for comparison with Dutch normative population and relatively long-term follow-up of up to 11 years.

\section{Conclusion}

This study shows comparable HRQOL of older patients with a stoma to older patients without a stoma or the normative population. Keeping in mind the severe impact that post-operative complications, in particular anastomotic leakage, can have on clinical recovery, a permanent stoma is feasible for elderly patients with a low situated rectal carcinoma. The negative impact of treatment on sexual functioning as found in the current study calls for further attention to alleviate this problem in sexually active patients. 


\section{References}

1. Allal AS, Bieri S, Pelloni A, et al. Sphincter-sparing surgery after preoperative radiotherapy for low rectal cancers: feasibility, oncologic results and quality of life outcomes. Br J Cancer 2000;82:1131-7.

2. Luna-Perez P, Rodriguez-Ramirez S, Hernandez-Pacheco F, Gutierrez de la BM, Fernandez R, Labastida S. Anal sphincter preservation in locally advanced low rectal adenocarcinoma after preoperative chemoradiation therapy and coloanal anastomosis. J Surg Oncol 2003;82:3-9.

3. Peeters KC, Tollenaar RA, Marijnen CA, et al. Risk factors for anastomotic failure after total mesorectal excision of rectal cancer. Br J Surg 2005;92:211-6.

4. Cornish JA, Tilney HS, Heriot AG, Lavery IC, Fazio VW, Tekkis PP. A meta-analysis of quality of life for abdominoperineal excision of rectum versus anterior resection for rectal cancer. Ann Surg Oncol 2007;14:2056-68.

5. Pachler J, Wille-Jorgensen P. Quality of life after rectal resection for cancer, with or without permanent colostomy. Cochrane Database Syst Rev 2005;(2): CD004323.

6. Bouvier AM, Jooste V, Bonnetain F, et al. Adjuvant treatments do not alter the quality of life in elderly patients with colorectal cancer: a population-based study. Cancer 2008;113:879-86.

7. Schmidt CE, Bestmann B, Kuchler T, Longo WE, Kremer B. Impact of age on quality of life in patients with rectal cancer. World J Surg 2005;29:190-7.

8. Janssen-Heijnen MLG, LW, van de Poll-Franse LV, et al. Results of 50 years cancer registry in the South of the Netherlands: 1955-2004 (in Dutch). 1-1-2005. Eindhoven, Eindhoven Cancer Registry. Ref Type: Generic

9. Thong MS, Mols F, Lemmens VE, et al. Impact of preoperative radiotherapy on general and diseasespecific health status of rectal cancer survivors: a population-based study. Int J Radiat Oncol Biol Phys 2011;81:e49-58.

10. van de Poll-Franse LV, Horevoorts N, van Eenbergen EM, et al. The patient reported outcomes following initial treatment and long term evaluation of survivorship registry: scope, rationale and design of an infrastructure for the study of physical and psychosocial outcomes in cancer survivorship cohorts. Eur J Cancer 2011;47:2188-94.

11. Aaronson NK, Muller M, Cohen PD, et al. Translation, validation, and norming of the Dutch language version of the SF-36 health survey in community and chronic disease populations. J Clin Epidemiol 1998;51:1055-68.

12. Sprangers MA, te Velde A, Aaronson NK. The construction and testing of the EORTC colorectal cancerspecific quality of life questionnaire module (QLQ-CR38). European organization for research and treatment of cancer study group on quality of life. Eur J Cancer 1999;35:238-47.

13. Sangha O, Stucki G, Liang MH, Fossel AH, Katz JN. The self-administered comorbidity questionnaire: a new method to assess comorbidity for clinical and health services research. Arthritis Rheum 2003;49: 156-63.

14. van Duijn C, Keij I. Sociaal-economische status indicator op postcode niveau. 50, 32-35. 2002. Maandstatistiek van de bevolking.

15. van de Poll-Franse LV, Mols F, Gundy CM, et al. Normative data for the EORTC QLQ-C30 and EORTCsexuality items in the general Dutch population. Eur J Cancer 2011;47:667-75.

16. Babyak MA. What you see may not be what you get: a brief, nontechnical introduction to overfitting in regression-type models. Psychosom Med 2004;66:411-21.

17. Clavien PA, Barkun J, de Oliveira ML, et al. The clavien-dindo classification of surgical complications: five-year experience. Ann Surg 2009;250:187-96.

18. Rutten HJ, den Dulk M, Lemmens VE, van de Velde CJ, Marijnen CA. Controversies of total mesorectal excision for rectal cancer in elderly patients. Lancet Oncol 2008;9:494-501.

19. Pucciarelli S, Del BP, Toppan P, et al. Health-related quality of life outcomes in disease-free survivors of mid-low rectal cancer after curative surgery. Ann Surg Oncol 2008;15:1846-54.

20. Rauch P, Miny J, Conroy T, Neyton L, Guillemin F. Quality of life among disease-free survivors of rectal cancer. J Clin Oncol 2004;22:354-60.

21. Sprangers MA, Schwartz CE. Integrating response shift into health-related quality of life research: a theoretical model. Soc Sci Med 1999;48:1507-15. 
22. Ho VP, Lee Y, Stein SL, Temple LK. Sexual function after treatment for rectal cancer: a review. Dis Colon Rectum 2011;54:113-25.

23. Bloemen JG, Visschers RG, Truin W, Beets GL, Konsten JL. Long-term quality of life in patients with rectal cancer: association with severe postoperative complications and presence of a stoma. Dis Colon Rectum 2009;52:1251-8.

24. Schmidt CE, Bestmann B, Kuchler T, Longo WE, Rohde V, Kremer B. Gender differences in quality of life of patients with rectal cancer. A five-year prospective study. World J Surg 2005;29:1630-41.

25. Sideris L, Zenasni F, Vernerey D, et al. Quality of life of patients operated on for low rectal cancer: impact of the type of surgery and patients' characteristics. Dis Colon Rectum 2005;48:2180-91. 


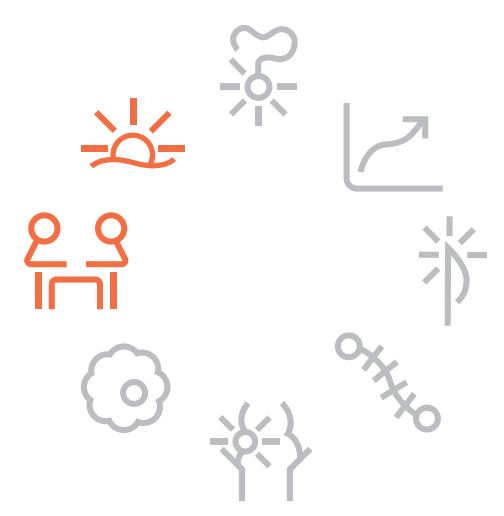



CHAPTER 8 Rectal cancer treatment in the elderly

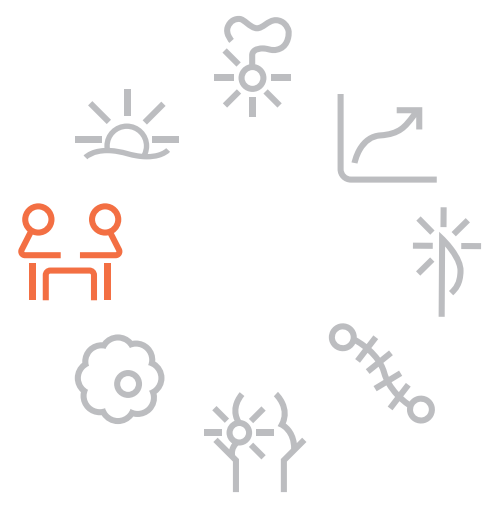

R.G. Orsini

S. Rostoft

H.J. Rutten

W.E. Longo et al. (eds.) Modern Management of Cancer of the Rectum 2nd ed., 2015. Springer-Verlag London. 385-403 


\section{Abstract}

Elderly rectal cancer patients are a heterogeneous group of patients ranging from the very fit to the frail patients who cannot sustain any treatment. Most elderly have comorbidities or present in an emergency situation which adversely affects outcome. In addition treatment related complications have more impact on outcome in elderly patients. Therefore a different approach compared to younger patients is needed. Only after a meticulous assessment of the physiological status and adequate counselling of the patient an individual treatment plan can be determined. Shared decision making is essential in the treatment of rectal cancer in the elderly. 


\section{Introduction}

In the Western population, with increasing life expectancy, there will be more and more patients affected by rectal cancer. Most of these patients are treated according to (inter)national guidelines or driven by expert meetings. But in the heterogeneous group called "elderly", ranging from very fit to very frail patients there is no consensus about the optimal therapy and multimodality therapy is less often used in this group. Overall, the elderly with rectal cancer are at risk for receiving under treatment. This chapter will give some guidance in assessing the physiological fitness of the elderly and determination of the optimal treatment for the individual patient.

\section{Frailty and performance of the elderly}

In elderly patients the physiological age can be a poor reflection of the chronological age. According to Balducci there are two chronological landmarks. ${ }^{1}$ The lower boundary of senescence is the age of 70, because between 70-75 years the incidence of age related changes increases sharply and 85 years may be considered as a red flag indicating a risk of frailty. Frailty is defined as "an elderly patient who is at heightened vulnerability to adverse health status change (such as hospitalization, mortality, nursing home admission) because of a multisystem reduction in reserve capacity", but it is still widely discussed how to identify frailty in an individual patient. ${ }^{2,3}$ In elderly with rectal cancer, identifying frailty is important because frailty is a predictor for post-operative complications, and frail patients have a shorter life-expectancy than non-frail patients. ${ }^{4,5}$ Figure 8.1 is an illustration on how the physiological reserve declines due to ageing and frailty.

In the period between the two previous mentioned landmarks, assessing the condition and vulnerability of the individual elderly can be very difficult. The most evidenced base process to detect and grade frailty for severity is a comprehensive geriatric assessment (CGA). ${ }^{6}$ A CGA has been shown to detect frailty in geriatric oncology patients. ${ }^{7-9}$ In a large prospective Norwegian study the CGA was used to predict post-operative complications and mortality in electively operated colorectal cancer patients $>70$ years. ${ }^{5}$ Severe comorbidity was predictive of severe complications, whereas instrumental activities of daily living dependency (IADL) and depression were predictive of any complication. ${ }^{10}$ Impaired nutrition and comorbidity predicted early mortality. The CGA evaluates several domains including functional status, mobility/risk of falls, cognition, depression, comorbidity, polypharmacy, social situation and geriatric syndromes. It has been suggested to categorize patients into three groups according to CGA results: fit patients, vulnerable patients and frail patients. ${ }^{11}$ 
$\%$ Organ function

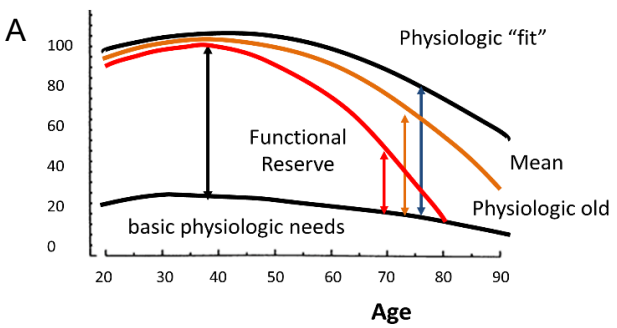

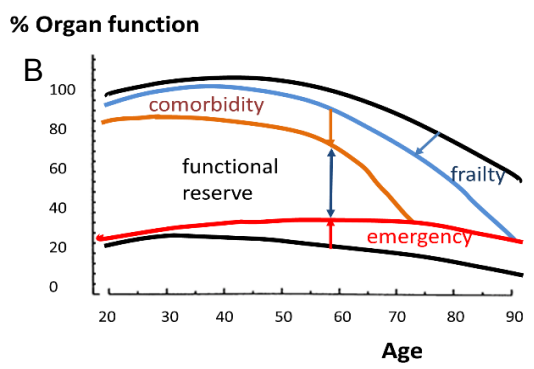

Figure 8.1 Physiological function and functional reserve. Figure A shows the natural decline of organ function (physiological function), basic physiologic needs and functional reserve. Figure $B$ shows the influence of co-morbidity on organ function and an emergency on basic physiologic needs. Frail patients have lower physiological function resulting in a small the functional reserve. In case of an emergency (such as surgery) a minor complication may deplete the functional reserve and increases the risk of worse outcome and mortality.

Other functional assessment-scores include the Barthel index and oncological performance status scales such as The Karnofsky performance status scale (KPS) and the Eastern Cooperative Oncology Group performance status (ECOG PS). The Barthel index is useful for assessing the functional ability of an elderly person at time of diagnosis. The KPS is validated in patients with cancer but is poorly validated in elderly patients as it does not include many areas of impaired functioning seen in the elderly. ${ }^{12}$ Furthermore, multiple studies have shown that for older cancer patients a CGA obtains additional information to the performance status and chronological age and has been proven feasible. $9,13,14$

Although a CGA may be used to detect frailty, it is time consuming and a resource intensive process. Particularly during a pre-operative outpatient visit, when the treatment options are discussed, normally not much time is left for assessing whether the patient is fit for (multimodality) treatment, and a CGA is too time consuming. Easier classifications such as the American Society of Anesthesiology classification (ASA) gives an estimation of a patient's anaesthetic risk, but high ASA-scores have not been proven to be predictive for post-operative morbidity and mortality. ${ }^{5,15}$ A test easily done during a pre-treatment visit is the timed-up-and-go test (TUG). It is a straightforward and quick test where the patient is observed and timed while he rises from an arm chair, walks 3 meters, turns, walks back and sits down again. Based on multiple studies the TUG is considered normal if a patients requires $\leq 20$ seconds to complete the test. ${ }^{16}$ In a multicenter cohort study containing onco-geriatric patients, twice as many patients were identified as at risk for post-operative complications using TUG compared to using ASA-classification. ${ }^{17}$ 
The Acute physiology and chronic health evaluation II (APACHE II) score is a surgical assessment scale and includes 12 physiological measurement including clinical and laboratory parameters. ${ }^{18}$ Although the APACHE II has a good prognostic capacity, it does not consider the nutritional status of the patient, which is particularly important in elderly patients. This test is relevant in the immediate post-operative period for both younger and elderly patients, but pre-operatively it provides little information about the suitability of elderly patient to undergo cancer treatment. ${ }^{12}$ Another surgical assessment scale in predicting morbidity and post-operative mortality in general surgery and patients with colorectal cancer, is the physiological and operative severity score for enumeration of mortality and morbidity (POSSUM) which contains twelve physiological and six operative variables. ${ }^{19}$ Unlike APACHE II, it contains information about cardiac arrhythmias and ECG findings. The intra operative score factors include information about the type and timing (emergency or elective) of the surgical procedure and if there is per-operative contamination. Both surgical assessment scales provide some information about pre-operative frailty, but focuses particularly on the post-operative period. They are useful in the pre-operative selection of elderly patients and prediction of morbidity and mortality, but they cannot be used as an exclusion for cancer treatment. ${ }^{12}$

Another way to assess the patient is the use of comorbidity scores, because comorbidity may predict a patient' physiological status and reaction to therapy. The adult comorbidity evaluation-27 (ACE-27), cumulative illness rating scale for geriatrics (CIRS-G) and the Charlson comorbidity index have been developed and validated in elderly patients with cancer. The CIRS-G is more time consuming than the Charlson comorbidity index, but both are considered as reliable tools to assess comorbidity. ${ }^{12,13}$ The International Society of Geriatric Oncology (SIOG) brought together a surgical risk assessment tool composed of geriatric assessment tools in order to obtain a comprehensive picture of the onco-geriatric patient. ${ }^{15}$ These tests were brought together in the PACE (Preoperative Assessment of Cancer in the Elderly). Included in the pace are; the Mini-mental state examination, Activities of daily living (ADL), Intstrumental acitivities of daily living (IADL), Geriatric depression scale, Brief fatigue inventory (BFI), ECOG PS, ASA score and Satariano's index of comorbidities. The PACE was validated in a prospective study where a 20-minute interview was administered within two weeks prior to planned surgery. In total 460 patients with a mean age of 76.9 years were included. They found that the likelihood of having a postoperative complication is increased by $50 \%$ when patients have a dependent IADL, abnormal ECOG PS or a moderate to severe BFI measured prior to surgery. Disability measured by a dependent ADL was found to best predict an extended hospital stay, but dependent IADL and abnormal ECOG PS were also significantly associated with prolonged hospital stay. Overall they recommend that PACE is used routinely in surgical practice and that it 
may be a valuable tool in the decision process concerning whether the elderly is a candidate for surgical intervention. ${ }^{15}$

In summary, there is still a lack of one easily applicable and validated assessment tool that offers a quick estimation of the patient's physiological fitness and is acceptable for surgeons, oncologists and geriatricians. Simple measures of objective performance status such as TUG are promising. ${ }^{20}$ Multiple studies have showed that the CGA seems to be the best assessment scale to help clinicians make difficult decisions in elderly patients. ${ }^{12,21}$ Identifying functional limitations, for example, highlights the need to offer exercise and resistance training to the patient prior to surgery. There is increasing evidence that this might decrease the rate of post-operative complications and improve survival. ${ }^{22,23}$ In addition, an assessment of cognitive function is also important when consulting with an older patient before any treatment is given. The number of patients with cognitive dysfunction increases with increasing age, and for the treating surgeon it is mandatory to realize whether the patient is capable of consenting to the treatment plan.

\section{Do we need to work-up the elderly differently?}

In the elderly it is important to bring in the life expectancy in the decision-making process. In the elderly there are huge differences in life expectancies comparing the fittest quartile of persons to the ones in the lowest quartile. For example, for woman who reached the age of 80 , the fittest quartile has a life expectancy of at least 13 years, while the sickest quartile has a life expectancy less than 4.6 years. ${ }^{24}$ For males who reached the age of 80 the fittest quartile has a life expectancy of at least 10 years and the lowest quartile less than 3.3 years. In the decision making process, it is important to estimate whether the patient belongs to the fittest quartile, the 50 percentile, or the sickest quartile. Life expectancy estimates can be used to determine if an elderly is likely to benefit from treatment. Patients who belong to the fittest $75 \%$ quartile are likely to benefit from the best oncological treatment, whereas in those patients who belong to the lowest quartile oncological outcome becomes less important. In those patients quality of life is more important and a less invasive approach can be desirable.

Overall increasing age and rectal cancer is associated with an increased likelihood of under treatment with local excision rather than radical surgery and multimodality therapy. This was confirmed in a small American population based study, where age also had a significant impact on whether patients received surgery alone or had surgery and chemo radiotherapy. Elderly who did receive the multimodality treatment had significantly better survival compared to elderly receiving suboptimal treatment. ${ }^{25}$ Another population based study among rectal cancer patients using the SEER registry also found a decreased use of any cancer treatment, a decreased use of radical surgery and an increased use of local excision. ${ }^{26}$ They also reported lower disease stages and 
decreasing survival rates as age increased. An explanation for the lower disease stage and survival could be due to surgical under treatment, as accurate staging is not possible with local excision.

An important aspect of rectal cancer treatment in the elderly is that most of these patients are affected by comorbidities. Figure 8.2 shows the patterns of different comorbid diseases in Dutch CRC patients according to age. In this population based sample $30 \%$ of patients $<60$ years suffered from comorbidities compared to $71 \%$ of the patients aged $>80$ years. ${ }^{27}$ In addition, a rising prevalence of comorbidities in all age groups was found during the study period. Regardless of age, having any comorbidity is associated with adverse outcome after colorectal surgery. ${ }^{28,29}$

European data showed improved colorectal cancer care in the elderly, with an increase in patients resected for cure, a decrease in post-operative mortality and improvement in stage of diagnosis. ${ }^{30}$ The Norwegian cancer registry-based study has also shown that the survival in rectal cancer patients aged 75 years or older have improved significantly from early (1994-1996) to late (2001-2003) time periods after implementing national management strategies. ${ }^{31}$ Knowing that the survival gap between young and elderly is closing, we are doing better.

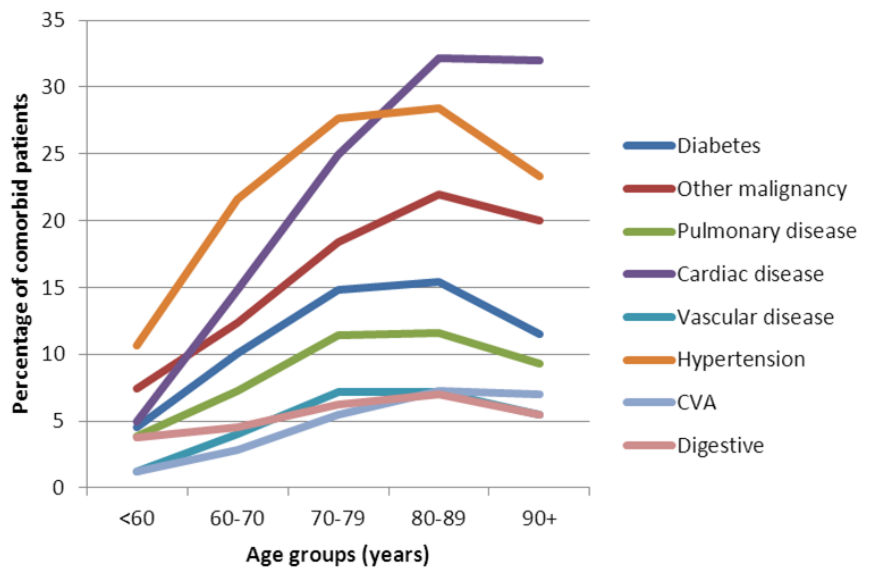

Figure 8.2 Prevalence of comorbidity in CRC patients. The figure presents the prevalence and distribution of specific comorbid diseases for different age groups. From Van Leersum et al. ${ }^{27}$

To lower the risk of under treatment, all elderly patients have to be adequately staged. It starts with optimal workup prior to any treatment given in the case of nonobstructive rectal cancer. ${ }^{32}$ For primary diagnosis and screening the endoscopy and full biopsy is the modality of choice. For further workup the diagnostic modality of choice is the Magnetic Resonance Imaging (MRI), given that it provides detailed images of the 
dissection planes, pelvic and mesorectal fascia. ${ }^{33}$ The mercury study group reported a good correlation with extra-mural spread and the MRI is able to predict a positive circumferential margin (CRM). ${ }^{34}$ With the use of Computed Tomography (CT) liver or pulmonary metastasis and enlarged intra-abdominal lymph nodes can be identified. The resolution of the $\mathrm{CT}$ is insufficient for accurate assessment of the dissection planes and CRM involvement. Endoscopic rectal ultrasound is sensitive enough to stage the depth of submocosal involvement, but cannot evaluate the mesorectal fascia as reliably as MRI. ${ }^{34,35}$

In case of obstructive rectal cancer or emergency surgery, surgeons have to be aware that it is better to place a deviating stoma and perform the resection in a more elective setting after adequate staging and neo-adjuvant treatment.

After accurate staging all elderly patients need to be discussed in a multi-disciplinary team (MDT) meeting. ${ }^{36,37}$ Recent studies have demonstrated a significant improvement in oncological outcome due to MDT meetings, resulting in decreased recurrence rates and increased survival. ${ }^{38,39}$ The factors influenced by MDT meetings and contributing to this improved oncological outcome include an increased number of patients undergoing an MRI in pre-operative staging, who received neo-adjuvant treatment, who underwent surgery for metastatic disease and who receive adjuvant therapy. ${ }^{38-41}$ Furthermore it can be advocated to involve a geriatrician in these MDT meetings because of the difficulty to assess the physiological fitness of the older patient. We believe that discussing such a patient in a MDT meeting will make it easier to assess whether the patient it fit for surgery and from which neo-adjuvant treatment the patient will benefits the most. In case of irresectable or metastatic disease, or if the patient is too frail for surgery, the MDT can discuss which palliative treatment suits the patients most properly.

\section{Neo-adjuvant treatment}

Elderly patients are underrepresented in clinical trials. Only $20-25 \%$ of the patients enrolled in clinical trials are 70 years and older. ${ }^{42,43}$ Large observational studies have also shown that aggressive treatment and the use of (neo)adjuvant treatment largely depends on the chronological age of the patient. ${ }^{21,44-47}$ Remarkably this was already reported in the early nineties ${ }^{48-50}$ but it seems that there has not been many changes. Even though fit older patients have been shown to tolerate cancer treatment and have additional survival benefit from (neo-)adjuvant therapies. ${ }^{32,51,52}$

Dutch population based data and data from the Dutch TME trial showed that elderly have better biological tumour response to neo-adjuvant radiotherapy than younger patients. ${ }^{53}$ Not only did the local recurrence rate decrease with neoadjuvant short course radiotherapy, but improvement was also seen in the distant metastatic rate and 
the cancer specific survival rate. In contrast, radiotherapy in younger patients only improves local control. Although the oncological response is better due to neo-adjuvant treatment, the overall survival rate has not improved. Elderly suffer from more complications compared to younger patients and the impact of complications is more severe. The increased mortality rate was not associated with neo-adjuvant treatment but directly attributable to surgery but this could also be due to power defect. Another population based study found a doubling of post-operative complications (especially deep infections and wound problems) in patients aged $\geq 75$ years who were treated with pre-operative radiotherapy compared with elderly not treated with radiotherapy. ${ }^{54}$ However, radiotherapy did not influence the 30 -day mortality rate and decreased the local recurrence rate.

A review by Martijn et al. including 9 RCT's and 10 population based samples, concluded that the best possible treatment should be given to all patients irrespective of age. ${ }^{55}$ This means the use of short course radiotherapy in case of primary resectable cancer and the use of long course chemo radiotherapy in case of locally advanced rectal cancer. With regard to toxicity they found no differences between young and older rectal cancer patients when modern radiotherapy techniques with small tissue volumes are used. Exceptions should only be if the patients' condition makes the patient unable to fulfil the combination of treatment. Another pooled analysis of 9 randomised controlled trials also showed that acute and late side effects of radiation therapy had the same toxicity in the elderly as the young. ${ }^{56}$ Other recent studies are less positive. A small study showed a high rate of treatment deviation in patients aged $\geq 75$ years. In their sample of 36 patients only $4(17 \%)$ did not deviate from the original treatment plan despite the ECOG performance scale of 0 or $1 .^{57}$

In the French ACCORD12/PRODIGE 2 trial patients were randomly assigned to 45Gy/25 fractions radiotherapy with concurrent Capecitabine or 50Gy/25 fractions radiotherapy and Capecitabine and Oxaliplatin. ${ }^{58}$ In a recent exploratory analysis the tolerance to treatment was investigated for elderly patients (aged $\geq 70$ years). ${ }^{59}$ Less elderly patients completed the radiotherapy treatment compared to patients $<70$ years $(4.2 \%$ vs. $1.4 \%$ $\mathrm{p}=0.03$ ). No differences were observed in the chemotherapy administration. In elderly patients there was a higher incidence of grade $3-4$ toxicity $(25.6 \%$ vs. $15.8 \% p=0.01)$, and fewer patients underwent surgery ( $95.8 \%$ vs. $99 \% \mathrm{p}=0.008)$.

Recent results from the Swedish Rectal Cancer Registry showed promising results regarding short course radiotherapy without direct curative surgery, but surgery was planned more than four weeks after completion of the radiotherapy. ${ }^{60}$ Tumour stage, lymph node positivity and circumferential involvement were significantly down staged. Pathologic complete response occurred in $8 \%$ of the patients and $11 \%$ had a higher TNM stage at pathological classification. Of all patients, $38 \%$ had post-operative 
complications, what is in line with larger samples. This study shows feasibility of shortcourse radiotherapy with a longer waiting period, particularly for older patients and patients with comorbidity, although younger patients could also fare well with this treatment regimen. To further investigate the effect on survival the Stockholm III trial is conducted. ${ }^{61}$ In this study patients are randomised in three groups; short-course radiotherapy with direct or delayed (4-8 weeks) surgery or long course radiotherapy with delayed surgery. This trial will give insight in the feasibility of this regimen and impact on local control and survival. Interim analyses showed that compliance was acceptable and severe acute toxicity was low. Immediate surgery after radiotherapy had a tendency to more complications, but only if the surgery was delayed beyond 10 days after completion of the radiotherapy. ${ }^{61}$

The main factors associated with a higher toxicity of chemotherapy are functional status impairment, dependency in ADL, depressive symptoms and polypharmacy. ${ }^{62}$ In elderly patients where there is doubt about the physiological fitness the administration of Capecitabine has been recommended. ${ }^{62-64}$ However in the FOCUS 2 study where only frail patients were included, it was associated with higher rates of grade 3-4 toxicity compared to infusional 5-FU/LV administration (37\% vs. 27\%). ${ }^{65} \mathrm{~A}$ review focussing on first line treatment strategies in elderly with metastatic CRC concluded that a combination therapy consists of Capecitabine and Oxaliplatin or Irinotecan ${ }^{64}$ should be $^{2}$ considered in older patients with adequate performance and functional status with reasonable life expectancy. The decision for Oxaliplatin or Irinotecan should be based on comorbidity, the drug specific toxicity and patients' wishes. For frail elderly, single agent of Capecitabine could be considered.

\section{Surgery and its morbidity}

Surgery is considered reasonably safe, also for most elderly patients, and surgery should not be denied on the basis of age alone. ${ }^{66,67}$ Whereas after elective surgery mortality rates will increase only minimally with age, in emergency surgery cases the mortality rates are higher. Emergency surgery is too burdensome in elderly, probably due to the limited functional reserve of many elderly ${ }^{1}$ and all efforts should be made to avoid emergency surgery. ${ }^{32,37}$ A study from the United Kingdom ${ }^{68}$ analysed 36767 non elective colorectal resections and divided patients in three age groups: 70-75 years, 7680 years and $>80$ years. Almost half of the patients received surgery for a malignancy and $21 \%$ for diverticulosis. The 30 -day mortality rates were $17 \%, 23 \%$ and $31 \%$, respectively, for the three age groups. Furthermore, one-year mortality was more than $50 \%$ in the oldest age group. In their population of non-elective cases, 1 in 4 cases aged 70 or older dies within 30-days of surgery indicating that non-elective surgery in elderly patients must be avoided. 
A Dutch study with non-elective colon resections found that in elderly patients with two or more additional risk factors, a non-elective resection should be considered a high-risk procedure with a mortality risk of up to $41 \% .{ }^{69}$ Another population based study ${ }^{29}$ found that emergency surgery in rectal cancer patients was associated with a higher risk of post-operative complications and increased mortality rates. Pre-operative pulmonary, cardiovascular or neurological comorbidity was also associated with postoperative morbidity and mortality.

A review, which included 28 of the 60 eligible studies investigated differences between young and elderly CRC patients analysing a total 34194 patients. ${ }^{70}$ Post-operative mortality in the 65-74 year age group was about 1.8 times higher compared to patients aged $<65$ years. For patients $75-84$ this was 3.2 times higher and 6.2 times higher in patients aged $85+$ years. These series include a mix of patients who underwent curative, palliative, emergency and elective surgery. Elderly patients were more often affected by respiratory complications when age increased. Patients aged 65-74 years were twice as likely to have respiratory complications compared to those $<65$ years, for older patients this rate rose up to 3 times as likely compared to those $<65$ years. No differences were seen according to age and the prevalence of anastomotic leakage (overall incidence rate $4.4 \%$ ). This could be due to using different definitions of this complication or the different methods of follow-up to detect this complication. Older patients were more likely to receive emergency surgery or no surgery. An increased frequency of comorbid condition in the elderly was seen, however in elderly patients undergoing surgery there was no evidence for increased morbidity in patients aged 85 and over, indicating the possibly careful selection of patients fit enough for surgery. Survival rates were reduced in elderly patients but in cancer specific survival agerelated differences were much less clear. Furthermore a large proportion of the elderly patients in this study survived for 2 of more years from surgery, irrespective of their age. This indicates the role of selection bias in the elderly.

Devon et al. ${ }^{71}$ investigated differences in CRC surgery outcomes between patients aged 50-74 years and patients aged 75 years and older. There were no differences in emergency surgery or palliative surgery between both groups. Elderly had more postoperative complications, especially cardiopulmonary compared to the younger age group. In hospital mortality rates were also higher in the elderly population $(4.2 \%$ vs. $1.0 \%)$. The 5-year overall survival rate was better in younger patients, but no differences were seen in the colorectal cancer specific survival rates at five years. The adjusted colorectal cancer hazard ratio was also not significantly different for patients aged 75 years and older compared to younger patients. Noteworthy, elderly patients in this study had lower disease stage at presentation compared to younger patients, indicating that elderly are less likely offered surgery for advanced cases. 
Advanced age is not a contra-indication for laparoscopic surgery in elderly patients and can be considered as safe. ${ }^{72}$ In a study among 535 patients with CRC, including 201 (37.6\%) patients aged 70 years or older patients were randomly assigned to receive open surgery or laparoscopic surgery. ${ }^{73}$ The elderly had higher ASA score compared to younger patients. In the elderly the laparoscopic procedure had significant lower morbidity rate $(20.2 \%$ vs. $37.5 \%, p=0.001)$ and decreased hospital stay ( 9.5 vs. 13.1 $\mathrm{p}=0.0001$ ) compared to elderly receiving open surgery. Interestingly, in the younger age group these differences were less pronounced. Other studies supports these findings where decreased morbidity, decreased hospital stay and earlier return of bowel function are seen after laparoscopic surgery compared to open surgery in elderly patients. $^{74-76}$

\section{Surgery and its mortality}

The 30-day mortality highly underestimates the risk of dying in the first year. ${ }^{77,78}$ In a study among 2131 patients who were operated with curative intent for stage I-III CRC the 30 -day mortality rate was $4.9 \%$, but rose to $12.4 \%$ in the first post-operative year. ${ }^{79}$ For rectal cancer patients risk factors for excess mortality in the first post-operative year were age $\geq 75$ years ( $R R 7.0 p=0,009)$, Charlson score of $>1(R R 5.2 p=0.01)$ and post-operative surgical complications (RR $5.9 p=0.02$ ). Patients aged $\geq 75$ years with rectal cancer had a one-year mortality rate of $15.6 \%$ compared to $6.8 \%$ for patients aged $65-74$ and $2.3 \%$ for patients $<65$ years. Another study showed doubling of the $30-$ day mortality rate already within 90 days post-surgery. ${ }^{78}$ In Figure 8.3 unpublished data of the Dutch Surgical Colorectal Audit (DSCA) of 44,202 patients demonstrate the relation between increasing age, comorbidity, emergency surgery and complications on 30-days mortality of colorectal cancer patients.

Results from the Dutch TME study combined with population based data showed that the 6-months post-operative mortality rate was significantly more increased in patients $\geq 75$ years compared with patients $<75$ years (Figure 8.4 ). ${ }^{80}$

A large Danish population based study with CRC patients found also increased mortality rates in elderly patients. ${ }^{81}$ Patients aged $76-80$ years had survival rates of $91 \%$ and $75 \%$, patients aged $81-85$ years had rates of $86 \%$ and $70 \%$ at 30-day and 6 months, respectively, compared to $94 \%$ and $81 \%$ in patients aged $61-75$ years. Regarding fiveyear relative survival rates, only minor differences were seen between the three age groups. 


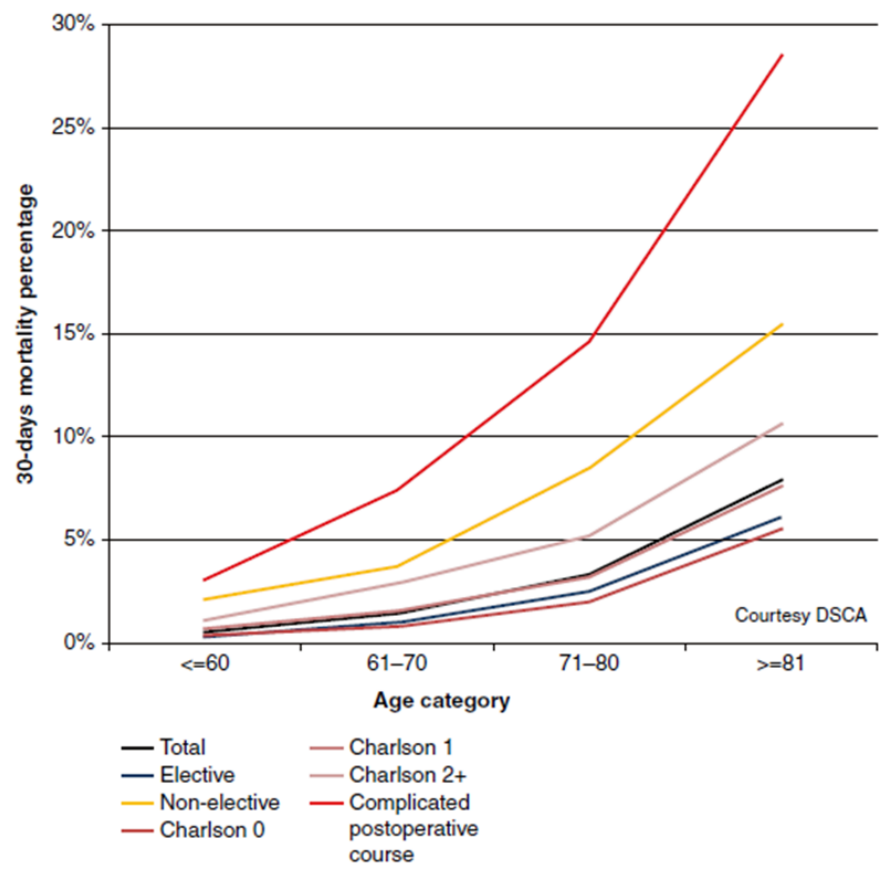

Figure 8.3 30-days mortality in colorectal cancer patients. Unpublished data of the DSCA demonstrating 30-days mortality in 44,202 registered colorectal cancer patients according to age and in relation to comorbidity, emergency operation and complicated post-operative course.

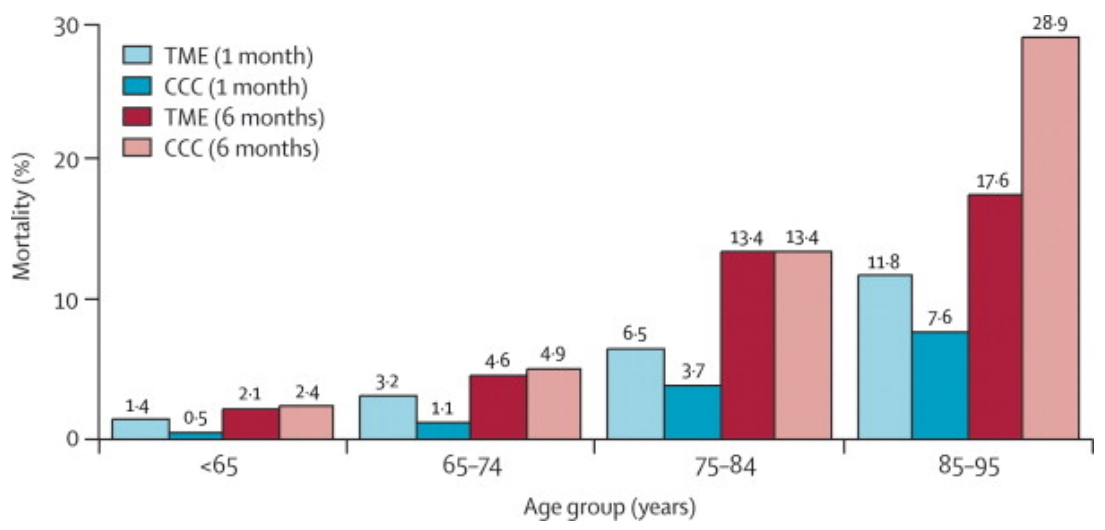

Figure 8.4 Post-operative mortality in rectal cancer patients. The figure presents the 1- month and 6-month mortality per age group in the Dutch TME study and the population-based Comprehensive Cancer Centre (CCC) databases, from Rutten et al. ${ }^{80}$ 
A French population based study showed that lowering the 30-day mortality rate from $18 \%$ to $8 \%$ led to a relative improvement of $27.5 \%$ in 5 -year survival. ${ }^{82}$ Other Dutch and Danish data also showed that the main difference in survival between young and elderly is due to the first post-operative year. ${ }^{77,81}$ Those elderly CRC patients who survive the first post-operative year have the same cancer related survival as younger patients. These findings emphasize the importance of post-operative care and after correction for post-operative mortality, survival in the elderly is not worse than in younger patients.

\section{Anastomosis or permanent stoma?}

Data from the Dutch TME study have shown that elderly patients are liable to more complications compared to younger patients. ${ }^{80}$ In addition the consequences of these complications are more severe. In their study anastomotic leakage occurred at similar rate but the ensuing mortality rate in younger patients was $8.2 \%$ compared to $57 \%$ in elderly patients. Other complications including abscesses, sepsis, pulmonary and cardiac complications were associated with an increased risk of dying within 6 months post-surgery in elderly patients.

A recent study using data from the Dutch Colorectal Audit including only colon resections also found that increasing age and anastomotic leakage is associated with increased mortality. ${ }^{83}$ Patients aged $65-80$ had an OR of 3.15 and patients aged $>80$ years an OR 5.16 for mortality after anastomotic leakage compared to younger patients.

These studies highlight the necessity to prevent major complications such as an anastomotic leakage in the elderly. To minimize the consequences of an anastomotic leakage a diverting stoma is placed. Interestingly, in the TME study up to $20 \%$ of the elderly patients did not have their stoma's reversed and in another study only half of the elderly patients had their stoma reversed at 18 months. ${ }^{84}$

In order to minimize post-operative morbidity and mortality due to anastomotic leakage in those patients at risk for complications, a permanent end colostomy may be considered. In the decision making process the assumed benefits of avoidance of a stoma have to be weighed against the potential life threatening complications of anastomotic leakage and the morbidity of stoma reversal. When patients are confronted with the choice between a permanent colostomy and restoration of bowel continuity, most will choose bowel continuity. We believe that a permanent stoma is feasible for elderly patients with a low situated rectal carcinoma, also in relation with health related quality of life (HRQL). In a population based sample with only low situated rectal cancer patients we found comparable HRQL between elderly patients with a permanent stoma and those with no stoma. ${ }^{85}$ In addition, in comparison with a 
normative population, no large differences were seen. These findings are supported by other studies where no relevant impact on HRQL of a permanent stoma was found. ${ }^{86,87}$ The low anterior resection syndrome (LARS), a complex of functional symptoms occurring after a low anterior resection, is frequently seen after a low anterior resection. In a large Danish study, ${ }^{88}$ using the LARS score, ${ }^{89}$ severe LARS was observed in $41 \%$ of all patients. The LARS increases morbidity, influences HRQL and results in poor functional outcome. Particularly in the elderly, the benefits of an anastomosis over a permanent stoma have to be weighed against the risks of anastomotic leakage or the morbidity of LARS.

\section{Post-operative care}

Surgery creates a similar stress response to a trauma including a hormonal, immunological, neurological and haematological response. ${ }^{90}$ These responses are essential for recovery. ${ }^{91}$ If these responses are not managed correctly peri-operatively, it is associated with poor outcome. For example, abnormalities of fluid and electrolyte balance may adversely affect organ function and surgical outcome and is associated with increased mortality and morbidity including cardio-respiratory complications, increased infections and wound healing complications. ${ }^{92-95}$ Furthermore, a positive fluid balance in elective colonic resections results in a delay in return of gastro-intestinal function and a prolonged hospital stay. ${ }^{96}$ Particularly elderly are pre-disposed to significant fluid and electrolyte abnormalities. Age-related pathophysiological changes, poor physiological reserves and/or polypharmacy make it difficult to manage an optimal physiological state and a zero fluid balance. Particularly in the first postoperative hours elderly and particularly frail patients should be monitored in the intensive care unit were vasopressin and inotropic agent can be given in order to maintain adequately organ tissue perfusion. Furthermore, post-operative care has to focus on complications, and aggressive assessment and treatment of these complications are needed. Particularly in the case of anastomotic leakage early and aggressive management may result in lower overall mortality. ${ }^{97}$

\section{Elderly and the role of local excision}

Minimally invasive approaches such as the Transanal Endoscopic Microsurgery (TEM) has been shown to have lower morbidity and compared to anterior resection. ${ }^{98}$ TEM is also considered as a safe technique in high risk patients and is well tolerated. ${ }^{99,100}$ For T1NO tumours, TEM has almost equal local recurrence rates as TME surgery. ${ }^{101}$ However, multiple studies have shown that TEM carries a higher risk of local recurrence particularly in T2-T3 NO tumours. ${ }^{102,103}$ 
A more recent development in neoadjuvant treatment is chemoradiotherapy. Promising results were found when patients with CT2-T3 tumours were treated with neoadjuvant chemoradiotherapy followed by TEM. ${ }^{104,105}$ In a study 70 T2NO patients were treated with long course neo-adjuvant CRT followed by a TEM or a laparoscopic anterior resection 6-8 weeks after the end of the CRT. In this study, similar local recurrence, distant metastasis and survival rates were found. ${ }^{104}$ In a study with cT2-cT3 patients after CRT, down staging was associated with low local recurrence rates and in patients with complete remission (ypT0) zero local recurrence was seen. ${ }^{105}$ In patients with ypT1 local recurrence rates of 0-6\% were seen and for ypT2 6-20\% local recurrence rates were observed. Another study containing 35 patients with pT2 tumours treated with TEM after long course radiotherapy found only one local recurrence in the followup period. ${ }^{106}$ The probability of surviving 8 years after treatment in this study was $83 \%$. However, in frail patients who are unfit for surgery the addition of chemotherapy to radiotherapy might be associated with increased morbidity. As mentioned earlier, elderly respond well to neo-adjuvant radiotherapy, so maybe there is a role in frail elderly for neo-adjuvant radiotherapy followed by a longer waiting period and complementary TEM surgery.

\section{The wait and see approach}

In some patients, there is a role for the wait and see approach after clinical complete response (CCR) after initial neo-adjuvant treatment. Habr-Gama included 71 patients with mainly CT3 tumours who had a CCR after chemoradiotherapy and found only two patients with local recurrence after a follow-up of 57 months. ${ }^{107}$ Of these two patients, one underwent a successful salvage operation. Three other patients developed distant metastasis during follow-up. In another study by Habr-Gama a local failure rate of $4.2 \%$, a 5 -year overall survival rate of $96 \%$ and a disease free survival rate of $72 \%$ were reported. $^{108}$

In the most recent Habr-Gama study, patients with cT2-4 N0-2 MO who had CCR 8 weeks after RCT (long course radiotherapy and 5-FU) were enrolled in a strict follow-up program with no immediate surgery. ${ }^{109}$ In their population $49 \%$ had CCR at initial assessment. Local recurrence developed in total in $31 \%$ of the patients in who salvage therapy was possible in $\geq 90 \%$ of these patients in both early and late recurrences. Of these 28 local recurrences, 17 were found within 12 months and 11 after 12 months. In total 17 patients (19\%) with cCR experienced unresectable (local or systemic) disease during follow-up.

A Dutch study is the only other study who also reported low recurrence and disease free survival rates. ${ }^{110}$ In total 21 patients were included in the wait and see policy 
group. The follow-up consisted of MRI, CT and colonoscopy. In this study only one patient had a local recurrence and received successful salvage surgery after a median follow-up of 25 months. The 2-year overall survival and 2-year disease free survival rates reported in this study were $100 \%$ and $89 \%$, respectively. Other studies have reported less promising results with local recurrence rates ranging from $23-83 \% .{ }^{111}$ The differences between reported local recurrence rates can be due to heterogeneity of the patient population, different interpretation of $\mathrm{CCR}$ on MRI or study design. Furthermore, most studies regarding this topic are Habr-Gama series. Glynne-jones and Huges concluded that at present evidence for the wait and see approach is insufficient, but the data has to be translated to the individual patient in the context of overall life expectancy. ${ }^{111}$ In addition, patients should be included in the decision making process in which evidence and tailored information is shared aimed to support those patients considering a wait and see approach.

In clinical trials, after pre-operative chemoradiotherapy a pCR is achieved in up to $25 \%$ of the patients. ${ }^{112}$ Furthermore, results from studies mentioned earlier are promising, but only in a minority of the patients and after careful patient selection surgery can be avoided. Future challenges lies in ways to achieve more pCR without increasing toxicity. A future treatment modality could be a more intensified radiotherapy treatment. Normally external beam radiotherapy is delivered with a total of 45-50.4 Gy divided over multiple fractions. Delivery of doses higher than $50 \mathrm{~Gy}$ is difficult without increasing morbidity due to the tolerance of normal tissue. The challenge is to increase the radiotherapy dose without increasing without increasing the morbidity. A possible and future modality could be endocavitary radiotherapy or a combination with external beam radiotherapy. Endocavitary or contact radiotherapy for the treatment of rectal cancer was first introduced in France by Lamarque and Gross in 1946 and was later popularised by Papillon in the period 1950-1990. ${ }^{113}$ In this period Papillon reported a 5-year survival rate of $75 \%$ after treating 300 patients with the "Papillon technique". More recent studies from the UK and France have shown feasibility for contact radiotherapy in selected cases and frail elderly patients. ${ }^{114,115}$ Another endocavitary approach is brachytherapy and has also shown promising results combined with contact radiotherapy or external beam radiotherapy with our without chemotherapy. ${ }^{116}$ Gerard concluded that endocavitary irradiation can be safely combined with external beam radiotherapy but radiotherapy without surgery should be restricted to highly selected patients such as frail patients or those refusing surgery. ${ }^{117}$

Surgery with neo-adjuvant treatment remains the standard of care for rectal cancer. In highly selected cases there is a role for a more conservative approach with radio- or chemo-radiotherapy without surgery. More studies and technical developments in this field are needed to guide the oncologists and surgeons in the selection of patients benefiting from non-operative management. 


\section{Stenting}

In selected cases with obstructive rectal cancer with poor prognosis a self-expending metal stenting (SEMS) can be a good alternative to surgery but this should always be discussed in a MDT. ${ }^{118,119}$ In a non-randomised prospective study a SEMS was successfully placed in $38 / 40$ patients, with a re-intervention rate of $19 \% .{ }^{120}$ Compared with the complication rate of $32 \%$ in those patients treated with surgery a SEMS can be considered as a good alternative to surgery. In a German study, 79\% of the patients found relief by a SEMS and 20 of the 37 patients died with a SEMS in place and required no surgical intervention. ${ }^{121}$ SEMS are associated with less risk, shorter hospital stay and less morbidity and mortality than surgical resection. ${ }^{122}$ The most devastating complication is a perforation that occurs in approximately $5 \%$ of the patients and surgical treatment in those cases is a high risk intervention. ${ }^{123}$

The EURECCA experts concluded that in case of palliative treatment of rectal cancer with a very poor prognosis (expected less than 3 months), due to pulmonary or liver metastases, self-expending metal stenting (SEMS) could be considered, as adjuvant to radiotherapy. ${ }^{124}$ In the curative treatment of rectal cancer, stent placement is inferior to emergency surgery in case of an acute obstruction and is associated with increased morbidity. ${ }^{125,126}$

\section{Tailored approach, quality of life $\&$ shared decision}

In the last decade, rectal cancer treatment has evolved from a surgery alone treatment to a multimodality and multidisciplinary treatment. Using international guidelines and guidelines from consensus meetings, we now know which treatment strategy is needed to achieve optimal oncological outcomes. In some cases, a very intensive treatment and extended resections are necessary in order to achieve the best oncological outcome. We have unpublished data that elderly have comparable HRQL after extended resections compared with elderly after non-extended resections in an series with particularly locally advanced cases. Other studies support the finding that elderly have comparable HRQL after treatment compared to younger patients. ${ }^{127,128}$ But it is known that some HRQL levels do not reach baseline levels even after 2-years post-surgery, which suggests that elderly will suffer a more permanent impaired physical function than younger counterparts. ${ }^{128}$ In the end it seems that elderly and particularly fit elderly have a good HRQL after treatment.

Different from treating young patients is that elderly can have other expectations of life making oncological outcome less important. In the young this may also be the case, but probably less commonly. This highlights the importance of shared decisions with a 
tailored approach in order to serve the patient' expectations of life and wishes. In shared decision-making, doctors and patients actively discuss and decide on therapeutic interventions, in order to reach a common goal instead of clinician driven goal based oncological outcome. ${ }^{129}$ This is extremely important in elderly patients in whom expectations of HRQL and for example a stoma play a more important role in the decision process than oncological outcome. Shared decision making is the cornerstone for adequate treatment in the elderly.

\section{Centralization and auditing}

We have tried to give some guidance in the treatment of rectal cancer. Nevertheless, rectal cancer treatment is complex and requires experiences in all fields of cancer care. In locally advanced cases multivisceral resections are often required and an experienced surgeon for these cases is necessary. Centralisation of these advanced cases will likely lead to more experience among varying disciplines at expert centers, positively influencing oncological outcomes due to improved rates of radical resections ${ }^{130}$ and lowered morbidity and mortality rates secondary to improved postoperative care. ${ }^{129,131}$ Also for elderly in whom there is doubt about the appropriate treatment given or in elderly with advanced cases referral to an expert center can mean a difference in outcome. In expert centers there is normally a high awareness for treatment and post-operative complications, therefore complications may be easier identified with more experience in the "know-how" to treat them.

We believe that centralization is mandatory in advanced rectal cancer cases, but this may also be the case in elderly rectal cancer patients. Why not centralise these patients? It can be advocated that the frail elderly do not benefit from centralisation if the travel distance is too far and travelling is too burdensome. But in these cases a consult with an expert center may be sufficient.

Because of the under representation of elderly patients in clinical trials it is difficult to extrapolate the results from trials to the older patients. It can be expected that randomised controlled trials involving elderly continue to be exceptional in future rectal cancer trials and studies. Why not use data from auditing and registries? The Dutch Colorectal audit is one of the most recent nation-wide audits on rectal cancer and has already shown an increased use of MRI, MDT meetings and pathologists reporting CRM involvement. ${ }^{132}$ In addition, less post-operative complications were seen and more radical resections were performed. With the use of auditing and registries, it may be possible to identify elderly patients at risk for under- or overtreatment, and from which therapeutic approach they benefit the most. This may lower the risk of doing more harm than good. 
When using international or expert meeting guidelines, registries or auditing there is no need to create a scale between countries or clinicians but it should be used as sharing expertise in an effort to make sure that every patient, including elderly are treated with the best cancer care.

\section{Conclusions}

The incidence of older patients with rectal cancer is rising. Therefore, elderly and frail patients with rectal cancer will be more commonly part of the daily practice. The most important aspect in treatment of elderly is the risk of under treatment. Clinicians have to be aware that older patients need proper staging with endoscopy, MRI and CT prior to any decision making or treatment given. When patients present with obstructive rectal cancer in an emergency setting, surgery has to focus on limiting complications, and therefore creation of a diverting stoma is the treatment of choice. After this procedure a full diagnostic work-up is still necessary followed by a MDT meeting. Prior to an MDT meeting it is useful to assess the physiological fitness of the patients primarily to know if the patients it fit enough for multi-modality treatment. Regardless of age, in the case of a fit elderly, we believe that treatment should be given according to international set guidelines and is the same as for younger patients. In cases where the clinician is in doubt about the condition of the elderly patient, a CGA may be useful and the geriatrician has to be involved in the treatment decision progress. Also in the elderly, the most important prognostic factor for survival is achieving a radical resection. In advanced cases, neo-adjuvant treatment is needed in order to achieve the best oncological outcome. Awareness of post-operative complications is especially important in elderly patients in whom complications results in higher post-operative mortality. We believe that it is mandatory to observe elderly patients an ICU postoperatively if their ASA score is $>2$. In these patients, early complications and disruption of the normal physiology may be appropriately handled with the use of vasopressin or inotropic agents in order to maintain a well-balanced fluid balance. Furthermore in elderly patients it can be considered to create a permanent colostomy in order to lower the ensuing mortality rate after an anastomotic leakage. In selected cases there is a role for the "wait and see" approach. For frail patients and elderly patients with nonresectable rectal cancer, treatment should focus on improving and prolonging a good quality of life. In such cases, a single agent chemotherapy or stenting could be the treatment of choice.

In the end, the crux of rectal cancer treatment in the elderly lies in shared decision making. Particularly the older patient knows clearly what he wants regarding HRQL and life expectancy. 


\section{Key points}

1. Age is an independent prognostic factor. However, a certain age for an individual patient is not relevant. Instead the physiologic changes that may have been caused in this patient are and should be known prior to decision making.

2. Co-morbidity is a prognostic variable which should be analysed carefully and optimised before commencement of any treatment.

3. Acute surgery should preferably be avoided or if necessary as minimised as possible

4. Elderly colorectal cancer patients represents a very heterogeneous group ranging from the very fit who are untitled to full oncological treatment to the very frail who cannot sustain any treatment. Elderly require individualised treatment which can only be decided after a meticulous assessment of the functional capacities

5. Existing guidelines may be evidence based, but cannot be validated for elderly in general and certainly not for the compromised elderly

6. If a elderly is considered physiological fit for treatment, neo-adjuvant treatment with radical resection is the treatment of choice.

7. Counselling the elderly patient should be from the perspective of the patients which may be quite different for expectations from a younger patients.

8. In elderly colorectal patients shared decision making is the cornerstone for adequate treatment. 


\section{References}

1. Balducci L. Geriatric oncology: challenges for the new century. Eur J Cancer 2000;36:1741-54.

2. Martin FC, Brighton P. Frailty: different tools for different purposes? Age Ageing 2008;37:129-31.

3. Bellan van KG, Rolland Y, Bergman H, Morley JE, Kritchevsky SB, Vellas B. The I.A.N.A Task Force on frailty assessment of older people in clinical practice. J Nutr Health Aging 2008;12:29-37.

4. Fried LP, Tangen CM, Walston J, et al. Frailty in older adults: evidence for a phenotype. J Gerontol A Biol Sci Med Sci 2001;56:M146-56.

5. Kristjansson SR, Nesbakken A, Jordhoy MS, et al. Comprehensive geriatric assessment can predict complications in elderly patients after elective surgery for colorectal cancer: a prospective observational cohort study. Crit Rev Oncol Hematol 2010;76:208-17.

6. Clegg A, Young J, Iliffe S, Rikkert MO, Rockwood K. Frailty in elderly people. Lancet 2013:381: 752-62.

7. Extermann M, Aapro M. Assessment of the older cancer patient. Hematol Oncol Clin North Am 2000;14: 63-ix.

8. Repetto L, Comandini D. Cancer in the elderly: assessing patients for fitness. Crit Rev Oncol Hematol 2000;35:155-60.

9. Repetto L, Fratino L, Audisio RA, et al. Comprehensive geriatric assessment adds information to Eastern Cooperative Oncology Group performance status in elderly cancer patients: an Italian Group for Geriatric Oncology Study. J Clin Oncol 2002;20:494-502.

10 Kristjansson SR, Jordhoy MS, Nesbakken A, et al. Which elements of a comprehensive geriatric assessment (CGA) predict post-operative complications and early mortality after colorectal cancer surgery? Journal of Geriatric Oncology 2010:1:57-65.

11. Repetto L, Venturino A, Fratino L, et al. Geriatric oncology: a clinical approach to the older patient with cancer. Eur J Cancer 2003;39:870-80.

12. Gosney MA. Clinical assessment of elderly people with cancer. Lancet Oncol 2005;6:790-7.

13. Extermann M, Overcash J, Lyman GH, Parr J, Balducci L. Comorbidity and functional status are independent in older cancer patients. J Clin Oncol 1998;16:1582-7.

14. Hurria A, Gupta S, Zauderer M, et al. Developing a cancer-specific geriatric assessment: a feasibility study. Cancer 2005;104:1998-2005.

15. Audisio RA, Pope D, Ramesh HS, et al. Shall we operate? Preoperative assessment in elderly cancer patients (PACE) can help. A SIOG surgical task force prospective study. Crit Rev Oncol Hematol 2008;65: 156-63.

16. Brouquet A, Cudennec T, Benoist S, et al. Impaired mobility, ASA status and administration of tramadol are risk factors for postoperative delirium in patients aged 75 years or more after major abdominal surgery. Ann Surg 2010;251:759-65.

17. Huisman MG, van Leeuwen BL, Ugolini G, et al. "Timed Up \& Go": a screening tool for predicting 30-day morbidity in onco-geriatric surgical patients? A multicenter cohort study. PLoS One 2014;9:e86863.

18. Knaus WA, Draper EA, Wagner DP, Zimmerman JE. APACHE II: a severity of disease classification system. Crit Care Med 1985;13:818-29.

19. Copeland GP, Jones D, Walters M. POSSUM: a scoring system for surgical audit. Br J Surg 1991;78: 355-60.

20. Robinson TN, Wu DS, Pointer L, Dunn CL, Cleveland JC, Jr., Moss M. Simple frailty score predicts postoperative complications across surgical specialties. Am J Surg 2013;206:544-50.

21. Chen RC, Royce TJ, Extermann M, Reeve BB. Impact of age and comorbidity on treatment and outcomes in elderly cancer patients. Semin Radiat Oncol 2012;22:265-71.

22. van Leeuwen BL, Huisman MG, Audisio RA. Surgery in older cancer patients - recent results and new techniques: worth the investment? Interdiscip Top Gerontol 2013,38, 124-131.

23. Liu JJ, Extermann M. Comprehensive geriatric assessment and its clinical impact in oncology. Clin Geriatr Med 2012;28:19-31.

24. Walter LC, Covinsky KE. Cancer screening in elderly patients: a framework for individualized decision making. JAMA 2001;285:2750-6.

25. Dharma-Wardene MW, de GC, Au HJ, Hanson J, Hatcher J. Ageism in rectal carcinoma? Treatment and outcome variations. Int J Gastrointest Cancer 2002;32:129-38. 
26. Chang GJ, Skibber JM, Feig BW, Rodriguez-Bigas M. Are we undertreating rectal cancer in the elderly? An epidemiologic study. Ann Surg 2007;246:215-21.

27. Van Leersum NJ, Janssen-Heijnen ML, Wouters MW, et al. Increasing prevalence of comorbidity in patients with colorectal cancer in the South of the Netherlands 1995-2010. Int J Cancer 2013;132: 2157-63.

28. Dekker JW, Gooiker GA, van der Geest LG, et al. Use of different comorbidity scores for risk-adjustment in the evaluation of quality of colorectal cancer surgery: does it matter? Eur J Surg Oncol 2012;38: 1071-8.

29. Janssen-Heijnen ML, Maas HA, Houterman S, Lemmens VE, Rutten HJ, Coebergh JW. Comorbidity in older surgical cancer patients: influence on patient care and outcome. Eur J Cancer 2007;43:2179-93.

30. Faivre J, Lemmens VE, Quipourt V, Bouvier AM. Management and survival of colorectal cancer in the elderly in population-based studies. Eur J Cancer 200743:2279-84.

31. Nedrebo BS, Soreide K, Eriksen MT, et al. Survival effect of implementing national treatment strategies for curatively resected colonic and rectal cancer. Br J Surg 2011:98:716-23.

32. Papamichael D, Audisio R, Horiot JC, et al. Treatment of the elderly colorectal cancer patient: SIOG expert recommendations. Ann Oncol 2009;20:5-16.

33. Tudyka V, Blomqvist L, Beets-Tan RG, et al. EURECCA consensus conference highlights about colon \& rectal cancer multidisciplinary management: The radiology experts review. Eur J Surg Oncol 2014;40: 469-75.

34. Extramural depth of tumor invasion at thin-section MR in patients with rectal cancer: results of the MERCURY study. Radiology 2007;243:132-9.

35. Taylor FG, Quirke P, Heald RJ, et al. One millimetre is the safe cut-off for magnetic resonance imaging prediction of surgical margin status in rectal cancer. Br J Surg 2011;98:872-9.

36. Extermann M. Integrating a geriatric evaluation in the clinical setting. Semin Radiat Oncol 2012; 2: 272-6.

37. Kristjansson SR, Farinella E, Gaskell S, Audisio RA. Surgical risk and post-operative complications in older unfit cancer patients. Cancer Treat Rev 2009;35:499-502.

38. Palmer G, Martling A, Cedermark B, Holm T. Preoperative tumour staging with multidisciplinary team assessment improves the outcome in locally advanced primary rectal cancer. Colorectal Dis 2011;13: 1361-9.

39. MacDermid E, Hooton G, MacDonald M, et al. Improving patient survival with the colorectal cancer multi-disciplinary team. Colorectal Dis 2009;11:291-5.

40. Augestad KM, Lindsetmo RO, Stulberg J, et al. International preoperative rectal cancer management: staging, neoadjuvant treatment, and impact of multidisciplinary teams. World J Surg 2010;34:2689-700.

41. Segelman J, Singnomklao $\mathrm{T}$, Hellborg $\mathrm{H}$, Martling A. Differences in multidisciplinary team assessment and treatment between patients with stage IV colon and rectal cancer. Colorectal Dis 2009;11:768-74.

42. Kohne $\mathrm{CH}$, Folprecht $\mathrm{G}$, Goldberg RM, Mitry E, Rougier P. Chemotherapy in elderly patients with colorectal cancer. Oncologist 2008:13:390-402.

43. Talarico L, Chen G, Pazdur R. Enrollment of elderly patients in clinical trials for cancer drug registration: a 7-year experience by the US Food and Drug Administration. J Clin Oncol 2004;22:4626-31.

44. Ayanian JZ, Zaslavsky AM, Fuchs CS, et al. Use of adjuvant chemotherapy and radiation therapy for colorectal cancer in a population-based cohort. J Clin Oncol 2003;21:1293-300.

45. Lemmens VE, Janssen-Heijnen ML, Verheij CD, Houterman S, Repelaer van Driel OJ, Coebergh JW. Comorbidity leads to altered treatment and worse survival of elderly patients with colorectal cancer. Br J Surg 2005;92:615-23.

46. Potosky AL, Harlan LC, Kaplan RS, Johnson KA, Lynch CF. Age, sex, and racial differences in the use of standard adjuvant therapy for colorectal cancer. J Clin Oncol 2002;20:1192-202.

47. Schrag D, Gelfand SE, Bach PB, Guillem J, Minsky BD, Begg CB. Who gets adjuvant treatment for stage II and III rectal cancer? Insight from surveillance, epidemiology, and end results--Medicare. J Clin Oncol 2001;19:3712-8.

48. Goodwin JS, Hunt WC, Samet JM. Determinants of cancer therapy in elderly patients. Cancer 1993; 72:594-601.

49. Newcomb PA, Carbone PP. Cancer treatment and age: patient perspectives. J Nat/ Cancer Inst 1993;85: 1580-4. 
50. Coburn MC, Pricolo VE, Soderberg $\mathrm{CH}$. Factors affecting prognosis and management of carcinoma of the colon and rectum in patients more than eighty years of age. J Am Coll Surg 1994;179;65-9.

51. Cohen SM, Neugut Al. Adjuvant therapy for rectal cancer in the elderly. Drugs Aging 2004;21;437-51.

52. Pallis AG, Papamichael D, Audisio R, et al. EORTC Elderly Task Force experts' opinion for the treatment of colon cancer in older patients. Cancer Treat Rev 2010;36:83-90.

53. Rutten $\mathrm{H}$, den Dulk $\mathrm{M}$, Lemmens $\mathrm{V}$, et al. Survival of elderly rectal cancer patients not improved: analysis of population based data on the impact of TME surgery. Eur J Cancer 2007;43;2295-300.

54. Maas HA, Lemmens VE, Nijhuis PH, de Hing IH, Koning CC, Janssen-Heijnen ML. Benefits and drawbacks of short-course preoperative radiotherapy in rectal cancer patients aged 75 years and older. Eur J Surg Oncol 2013;39:1087-93.

55. Martijn H, Vulto JC. Should radiotherapy be avoided or delivered differently in elderly patients with rectal cancer? Eur J Cancer 2007;43:2301-6.

56. Pignon $\mathrm{T}$, Horiot JC, Bolla $\mathrm{M}$, et al. Age is not a limiting factor for radical radiotherapy in pelvic malignancies. Radiother Oncol 1997;42:107-20.

57. Margalit DN, Mamon HJ, Ancukiewicz M, et al. Tolerability of combined modality therapy for rectal cancer in elderly patients aged 75 years and older. Int J Radiat Oncol Biol Phys 2011;81:e735-41.

58. Gerard JP, Azria D, Gourgou-Bourgade S, et al. Comparison of two neoadjuvant chemoradiotherapy regimens for locally advanced rectal cancer: results of the phase III trial ACCORD 12/0405-Prodige 2. J Clin Oncol 2010;28:1638-44.

59. Francois E, Azria D, Gourgou-Bourgade S, et al. Results in the elderly with locally advanced rectal cancer from the ACCOR12/PRODIGE 2 phase III trial: Tolerance and efficacy. Radiother Oncol 2014;110:144-9.

60. Pettersson D, Holm T, Iversen H, Blomqvist L, Glimelius B, Martling A. Preoperative short-course radiotherapy with delayed surgery in primary rectal cancer. Br J Surg 2012;99:577-83.

61. Pettersson D, Cedermark B, Holm T, et al. Interim analysis of the Stockholm III trial of preoperative radiotherapy regimens for rectal cancer. Br J Surg 2010;97:580-7.

62. Feliu J, Sereno M, Castro JD, Belda C, Casado E, Gonzalez-Baron M. Chemotherapy for colorectal cancer in the elderly: Whom to treat and what to use. Cancer Treat Rev 2009;35:246-54.

63. Lichtman SM. Management of advanced colorectal cancer in older patients. Oncology (Williston Park) 2005;19:597-602.

64. Meulenbeld HJ, Creemers GJ. First-line treatment strategies for elderly patients with metastatic colorectal cancer. Drugs Aging 2007;24:223-38.

65. Seymour MT, Thompson LC, Wasan HS, et al. Chemotherapy options in elderly and frail patients with metastatic colorectal cancer (MRC FOCUS2): an open-label, randomised factorial trial. Lancet 2011;377: 1749-59.

66. Berger DH, Roslyn JJ. Cancer surgery in the elderly. Clin Geriatr Med 1997;13:119-41.

67. Kemeny MM, Busch-Devereaux E, Merriam LT, O'Hea BJ. Cancer surgery in the elderly. Hematol Oncol Clin North Am 2000;14:169-92.

68. Mamidanna R, Eid-Arimoku L, Almoudaris AM, et al. Poor 1-year survival in elderly patients undergoing nonelective colorectal resection. Dis Colon Rectum 2012;55:788-96.

69. Kolfschoten NE, Wouters MW, Gooiker GA, et al. Nonelective colon cancer resections in elderly patients: results from the dutch surgical colorectal audit. Dig Surg 2012;29:412-9.

70. Surgery for colorectal cancer in elderly patients: a systematic review. Colorectal Cancer Collaborative Group. Lancet 2000;356:968-74.

71. Devon KM, Vergara-Fernandez O, Victor JC, McLeod RS. Colorectal cancer surgery in elderly patients: presentation, treatment, and outcomes. Dis Colon Rectum 2009;52:1272-7.

72. Schwandner O, Schiedeck TH, Bruch HP. Advanced age--indication or contraindication for laparoscopic colorectal surgery? Dis Colon Rectum 1999;42:356-62.

73. Frasson M, Braga M, Vignali A, Zuliani W, Di Carlo V. Benefits of laparoscopic colorectal resection are more pronounced in elderly patients. Dis Colon Rectum 2008;51:296-300.

74. Feng $\mathrm{B}$, Zheng $\mathrm{MH}$, Mao $\mathrm{ZH}$, et al. Clinical advantages of laparoscopic colorectal cancer surgery in the elderly. Aging Clin Exp Res 2006;18:191-5.

75. Law WL, Chu KW, Tung PH. Laparoscopic colorectal resection: a safe option for elderly patients. J Am Coll Surg 2002;195:768-73. 
76. Vignali A, Di PS, Tamburini A, Radaelli G, Orsenigo E, Staudacher C. Laparoscopic vs. open colectomies in octogenarians: a case-matched control study. Dis Colon Rectum 2005;48:2070-5.

77. Dekker JW, van den Broek CB, Bastiaannet E, van de Geest LG, Tollenaar RA, Liefers GJ. Importance of the first postoperative year in the prognosis of elderly colorectal cancer patients. Ann Surg Oncol 2011; 18:1533-9.

78. Visser BC, Keegan H, Martin M, Wren SM. Death after colectomy: it's later than we think. Arch Surg 2009;144:1021-7.

79. Gooiker GA, Dekker JW, Bastiaannet E, et al. Risk factors for excess mortality in the first year after curative surgery for colorectal cancer. Ann Surg Oncol 2012;19:2428-34.

80. Rutten HJ, den Dulk M, Lemmens VE, van de Velde CJ, Marijnen CA. Controversies of total mesorectal excision for rectal cancer in elderly patients. Lancet Oncol 2008;9:494-501.

81. Iversen LH, Pedersen L, Riis A, Friis S, Laurberg S, Sorensen HT. Age and colorectal cancer with focus on the elderly: trends in relative survival and initial treatment from a Danish population-based study. Dis Colon Rectum 2005;48:1755-63.

82. Mitry E, Bouvier AM, Esteve J, Faivre J. Benefit of operative mortality reduction on colorectal cancer survival. Br J Surg 2002;89:1557-62.

83. Bakker IS, Grossmann I, Henneman D, Havenga K, Wiggers T. Risk factors for anastomotic leakage and leak-related mortality after colonic cancer surgery in a nationwide audit. Br J Surg 2014;101:424-32.

84. den Dulk M, Smit M, Peeters KC, et al. A multivariate analysis of limiting factors for stoma reversal in patients with rectal cancer entered into the total mesorectal excision (TME) trial: a retrospective study. Lancet Oncol 2007;8:297-303.

85. Orsini RG, Thong MS, van de Poll-Franse LV, et al. Quality of life of older rectal cancer patients is not impaired by a permanent stoma. Eur J Surg Oncol 2013;39:164-70.

86. Cornish JA, Tilney HS, Heriot AG, Lavery IC, Fazio VW, Tekkis PP. A meta-analysis of quality of life for abdominoperineal excision of rectum versus anterior resection for rectal cancer. Ann Surg Oncol 2007; 14:2056-68.

87. Pachler J, Wille-Jorgensen P. Quality of life after rectal resection for cancer, with or without permanent colostomy. Cochrane Database Syst Rev 2012,12, CD004323.

88. Bregendahl S, Emmertsen KJ, Lous J, Laurberg S. Bowel dysfunction after low anterior resection with and without neoadjuvant therapy for rectal cancer: a population-based cross-sectional study. Colorectal Dis 2013;15:1130-9.

89. Emmertsen KJ, Laurberg S. Low anterior resection syndrome score: development and validation of a symptom-based scoring system for bowel dysfunction after low anterior resection for rectal cancer. Ann Surg 2012;255:922-8.

90. Desborough JP. The stress response to trauma and surgery. Br J Anaesth 2000;85:109-17.

91. El-Sharkawy AM, Sahota O, Maughan RJ, Lobo DN. The pathophysiology of fluid and electrolyte balance in the older adult surgical patient. Clin Nutr 2014;33:6-13.

92. Arieff Al. Fatal postoperative pulmonary edema: pathogenesis and literature review. Chest 1999;115: 1371-7.

93. Lobo DN, Macafee DA, Allison SP. How perioperative fluid balance influences postoperative outcomes. Best Pract Res Clin Anaesthesiol 2006;20:439-55.

94. Holte K, Sharrock NE, Kehlet H. Pathophysiology and clinical implications of perioperative fluid excess. BrJ Anaesth 2002;89:622-32.

95. Payen D, de Pont AC, Sakr Y, Spies C, Reinhart K, Vincent JL. A positive fluid balance is associated with a worse outcome in patients with acute renal failure. Crit Care 2008;12:R74.

96. Lobo DN, Bostock KA, Neal KR, Perkins AC, Rowlands BJ, Allison SP. Effect of salt and water balance on recovery of gastrointestinal function after elective colonic resection: a randomised controlled trial. Lancet 2002:359:1812-8.

97. Khan AA, Wheeler JM, Cunningham C, George B, Kettlewell M, Mortensen NJ. The management and outcome of anastomotic leaks in colorectal surgery. Colorectal Dis 2008;10:587-92.

98. Winde G, Nottberg H, Keller R, Schmid KW, Bunte H. Surgical cure for early rectal carcinomas (T1). Transanal endoscopic microsurgery vs. anterior resection. Dis Colon Rectum 1996;39:969-76.

99. Dafnis G, Pahlman L, Raab Y, Gustafsson UM, Graf W. Transanal endoscopic microsurgery: clinical and functional results. Colorectal Dis 2004;6:336-42. 
100. Endreseth BH, Wibe A, Svinsas M, Marvik R, Myrvold HE. Postoperative morbidity and recurrence after local excision of rectal adenomas and rectal cancer by transanal endoscopic microsurgery. Colorectal Dis 2005;7:133-7.

101. Lee $\mathrm{W}$, Lee $\mathrm{D}$, Choi S, Chun $\mathrm{H}$. Transanal endoscopic microsurgery and radical surgery for T1 and T2 rectal cancer. Surg Endosc 2003;17:1283-7.

102. Paty PB, Nash GM, Baron P, et al. Long-term results of local excision for rectal cancer. Ann Surg 2002; 236:522-9.

103. Mellgren A, Sirivongs $P$, Rothenberger DA, Madoff RD, Garcia-Aguilar J. Is local excision adequate therapy for early rectal cancer? Dis Colon Rectum 2000;43:1064-71.

104. Lezoche $G$, Baldarelli $M$, Guerrieri $M$, et al. A prospective randomized study with a 5-year minimum follow-up evaluation of transanal endoscopic microsurgery versus laparoscopic total mesorectal excision after neoadjuvant therapy. Surg Endosc 2008;22:352-8.

105. Borschitz T, Wachtlin D, Mohler M, Schmidberger $H$, Junginger T. Neoadjuvant chemoradiation and local excision for T2-3 rectal cancer. Ann Surg Oncol 2008;15:712-20.

106. Lezoche E, Guerrieri M, Paganini AM, Feliciotti F. Long-term results of patients with pT2 rectal cancer treated with radiotherapy and transanal endoscopic microsurgical excision. World J Surg 2002;26: 1170-4.

107. Habr-Gama A, Perez RO, Nadalin W, et al. Long-term results of preoperative chemoradiation for distal rectal cancer correlation between final stage and survival. J Gastrointest Surg 2005;9:90-9.

108. Habr-Gama A, Perez RO, Sao Juliao GP, Proscurshim I, Gama-Rodrigues J. Nonoperative approaches to rectal cancer: a critical evaluation. Semin Radiat Oncol 2011;21:234-9.

109. Habr-Gama A, Gama-Rodrigues J, Sao Juliao GP, et al. Local recurrence after complete clinical response and watch and wait in rectal cancer after neoadjuvant chemoradiation: Impact of salvage therapy on local disease control. Int J Radiat Oncol Biol Phys 2014;88:822-8.

110. Maas M, Beets-Tan RG, Lambregts DM, et al. Wait-and-see policy for clinical complete responders after chemoradiation for rectal cancer. J Clin Oncol 2011;29:4633-40.

111. Glynne-Jones R, Hughes R. Critical appraisal of the 'wait and see' approach in rectal cancer for clinical complete responders after chemoradiation. Br J Surg 2012;99:897-909.

112. O'Neill BD, Brown G, Heald RJ, Cunningham D, Tait DM. Non-operative treatment after neoadjuvant chemoradiotherapy for rectal cancer. Lancet Oncol 2007;8:625-33.

113. Papillon J. Present status of radiation therapy in the conservative management of rectal cancer. Radiother Oncol 1990;17:275-83.

114. Gerard JP, Chapet O, Ortholan C, Benezery K, Barbet N, Romestaing P. French experience with contact X-ray endocavitary radiation for early rectal cancer. Clin Oncol (R Coll Radiol) 2007;19:661-73.

115. Sun MA, Grieve RJ, McDonald AC, et al. Combined modality treatment of early rectal cancer: the UK experience. Clin Oncol (R Coll Radiol) 2007;19:674-81.

116. Sun MA, Lee CD, Snee AJ, Perkins K, Jelley FE, Wong H. High dose rate brachytherapy as a boost after preoperative chemoradiotherapy for more advanced rectal tumours: the Clatterbridge experience. Clin Oncol (R Coll Radiol) 2007;19:711-19.

117. Gerard JP, Romestaing P, Chapet O. Radiotherapy alone in the curative treatment of rectal carcinoma. Lancet Oncol 2003;4:158-66.

118. Cirocchi R, Farinella E, Trastulli S, et al. Safety and efficacy of endoscopic colonic stenting as a bridge to surgery in the management of intestinal obstruction due to left colon and rectal cancer: a systematic review and meta-analysis. Surg Oncol 2013;22:14-21.

119. Suarez J, Jimenez J, Vera R, et al. Stent or surgery for incurable obstructive colorectal cancer: an individualized decision. Int J Colorectal Dis 2010;25:91-6.

120. Baron TH. Indications and results of endoscopic rectal stenting. J Gastrointest Surg 2004;8:266-9.

121. Hunerbein M, Krause M, Moesta KT, Rau B, Schlag PM. Palliation of malignant rectal obstruction with self-expanding metal stents. Surgery 2005 Jan,137(1), 42-47.

122. Ronnekleiv-Kelly SM, Kennedy GD. Management of stage IV rectal cancer: palliative options. World J Gastroenterol 2011;17:835-47.

123. Watt AM, Faragher IG, Griffin TT, Rieger NA, Maddern GJ. Self-expanding metallic stents for relieving malignant colorectal obstruction: a systematic review. Ann Surg 2007;246:24-30. 
124. van de Velde CJ, Aristei C, Boelens PG, et al. EURECCA colorectal: multidisciplinary mission statement on better care for patients with colon and rectal cancer in Europe. Eur J Cancer 2013;49:2784-90.

125. Breitenstein S, Rickenbacher A, Berdajs D, Puhan M, Clavien PA, Demartines N. Systematic evaluation of surgical strategies for acute malignant left-sided colonic obstruction. Br J Surg 2007;94:1451-60.

126. van Hooft JE, Bemelman WA, Oldenburg B, et al. Colonic stenting versus emergency surgery for acute left-sided malignant colonic obstruction: a multicentre randomised trial. Lancet Oncol 2011;12:344-52.

127. Mastracci TM, Hendren S, O'Connor B, McLeod RS. The impact of surgery for colorectal cancer on quality of life and functional status in the elderly. Dis Colon Rectum 2006;49:1878-84.

128. Schmidt CE, Bestmann B, Kuchler T, Longo WE, Kremer B. Impact of age on quality of life in patients with rectal cancer. World J Surg 2005;29:190-7.

129. Vermeer TA, Orsini RG, Rutten HJ. Surgery for Rectal Cancer-What is on the Horizon? Curr Oncol Rep 2014;16:372.

130. McArdle CS, Hole DJ. Influence of volume and specialization on survival following surgery for colorectal cancer. Br J Surg 2004;91:610-7.

131. Borowski DW, Kelly SB, Bradburn DM, Wilson RG, Gunn A, Ratcliffe AA. Impact of surgeon volume and specialization on short-term outcomes in colorectal cancer surgery. Br J Surg 2007;94:880-9.

132. Van Leersum NJ, Snijders HS, Henneman D, et al. The Dutch surgical colorectal audit. Eur J Surg Oncol 2013;39:1063-70. 
CHAPTER 9 Surgery for rectal cancer-what is on the horizon?

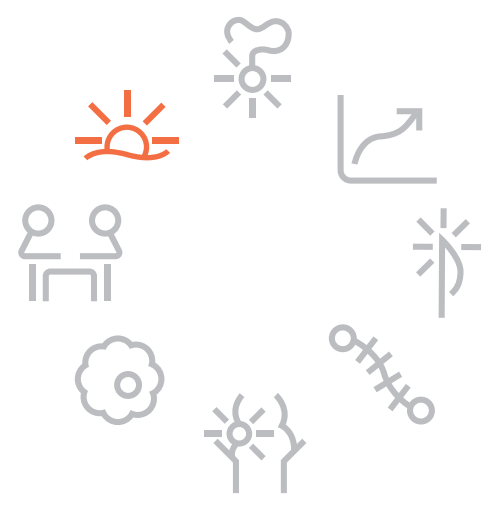

T.A. Vermeer* R.G. Orsini* H.J. Rutten 


\section{Abstract}

The management of rectal cancer has improved considerably in recent decades. Surgery remains the cornerstone of the treatment. However, the role of preoperative imaging has made it possible to optimize the treatment plan in rectal patients. Neoadjuvant treatment may be indicated in efforts to sterilize possible tumor deposits outside the surgical field, or may be used to downsize and downstage the tumor itself. The optimal sequence of treatment modalities can be determined by a multidisciplinary team, who not only use pre-treatment imaging, but also review pathologic results after surgery. The pathologist plays a pivotal role in providing feedback about the success of surgery, i.e., the distance between the tumor and the circumferential resection margin, the quality of surgery, and the effect of neoadjuvant treatment. Registry and auditing of all treatment variables can further improve outcomes. In this century, rectal cancer treatment has become a team effort. 
Over the past few decades, the treatment of rectal cancer has evolved significant, changing from a surgery-alone treatment to a disease approached with multidisciplinary, multimodality treatments. Improvements have been accomplished in all disciplines involved in the treatment of this disease. Therefore, the contemporary approach of multimodality management should allow rectal cancer patients to benefit from these achievements. The question now is not so much which approach is preferred, but rather how can we deliver a certain quality of treatment and standard of care to any patient with rectal cancer, irrespective of geographic locale.

Total mesorectal excision (TME), based on anatomical and embryological studies as described by Heald in 1986, is still the cornerstone of current rectal cancer surgery. ${ }^{1}$ The modern surgical approach of rectal cancer is still based on the importance of the distance of the radial tumor border to the mesorectal facia (MRF), as described by Quirke decades ago. ${ }^{2}$ This radial margin, called the circumferential resection margin (CRM), has become the most important indicator of the quality of rectal cancer surgery, since a positive resection margin correlates with poor disease related outcomes, i.e., corresponding increase in local recurrence, distant metastasis, and worsened survival. ${ }^{3,4}$ Magnetic resonance imaging (MRI) is the imaging modality of choice, given that it provides detailed images of dissection planes and pelvic fascia, specifically allowing evaluation of the MRF in rectal cancer. The Mercury Study Group reported a good correlation of extramural spread of rectal tumours on MRI and subsequent histopathology, confirming the reliability of MRI in predicting local tumour invasion. ${ }^{5}$ However, the true value of MRI lies in its ability to predict MRF involvement, i.e., to predict a positive CRM. The accepted cut-off distance for predicting MRF involvement using MRI is $1 \mathrm{~mm}$ between the tumor and mesorectal fascia. Using a cut-off of $<1 \mathrm{~mm}$, patients with larger margins had local recurrence rates below $9 \%$, whereas patients with a distance of $<1 \mathrm{mmon}$ MRI have shown an approximate $20 \%$ risk of developing local recurrence. ${ }^{6}$ With the use of computed tomography (CT), liver or pulmonary metastases and enlarged intra-abdominal lymph nodes can be identified. Although CT is the diagnostic modality of choice in colon cancer, the resolution is insufficient for the accurate assessment of surgical resection plane involvement in rectal cancer. Endoscopic rectal ultrasound (EUS) is sensitive enough to stage the depth of submucosal involvement, but cannot evaluate the mesorectal fascia as reliably as MRI. ${ }^{7}$ Therefore, rectal ultrasound is the diagnostic tool of choice in differentiating between early $\mathrm{T}$ stages.

Neoadjuvant radiation treatment in rectal cancer is very effective in improving diseaserelated outcomes in selected patients. The Dutch TME trial has demonstrated that in patients in whom a negative resection margin is feasible, $5 \times 5$ Gy short-course radiotherapy followed by immediate surgery is associated with good oncological results, improving local control rates above and beyond that which could be achieved 
with the use of TME alone. ${ }^{8}$ However, tumors threatening or even invading the mesorectal fascia require a different treatment strategy. In order to facilitate a complete radical resection, downsizing and even downstaging the tumor with a long course of preoperative (chemo-) radiation is effective, as has been shown in several randomized studies. ${ }^{9,10}$ Similarly, all locally recurrent rectal cancers (LRRC) should be considered as locally advanced, given that these recurrences frequently occur in areas not delineated by an anatomical fascia and therefore easily invade surrounding tissue or organs. The assessment and management of locally advanced rectal cancer (LARC) and LRRC are complex and are generally not comparable to conventional rectal cancer treatment due to the heterogeneity of these patients. The "Beyond TME

Collaborative" is a worldwide group of experts in the treatment of LARC and LRRC who provided a framework for the diagnostic evaluation and treatment of these complex patients. ${ }^{11}$ Preoperative chemoradiation (CRT) is the accepted neoadjuvant treatment for advanced rectal cancer patients. The effect of CRT is based on the sterilization of lateral lymphatic spread, which is not removed during surgery, and the downsizing and downstaging of the tumor. Chemoradiation is followed by a timed break to allow continued disease shrinkage and downstaging, thereby improving resectability and disease-free survival. An interval $\geq 8$ weeks between chemoradiation and surgery is safe and associated with a higher rate of pathological complete response ( $\mathrm{PCR})$, decreased local recurrence, and improved survival outcomes. ${ }^{12,13}$ All of these improvements in both diagnostics and therapy have resulted in instruments that maximize cure rates in patients with non-metastatic disease. The reality, however, is that wide variations in rectal cancer treatment approaches and oncological outcomes on a national and international level exist, as revealed by large population-based studies. ${ }^{14,15}$

To date, patient still remain dependent on the expertise and personal preferences of the surgeon involved in their care. The day-to-day clinical practice is not always evidence-based due to personal preferences of the surgeon, slow implementation of guidelines, lack of evidence for specific treatments, or inadequate infrastructure. ${ }^{16}$ Therefore, registration, centralization, auditing, and multidisciplinary team meetings are important tools to facilitate optimal rectal cancer treatment for each individual on an international level and eliminate shortcomings in clinical practice.

\section{The multidisciplinary approach to rectal cancer}

A multidisciplinary European approach to the treatment of rectal cancer started around 1995 following a publication by Calman and Hine. In order to enable every patient to receive the same high quality and uniform standard care, these authors introduced a framework for the commissioning of cancer services in England and Wales. ${ }^{17}$ The 
evolution of the treatment of colorectal cancer over the last few decades led to the implementation of multidisciplinary teams (MDT's) in many European countries. At this point in time, MDT's are an established part of colorectal cancer treatment and have been implemented in almost all European cancer centers. All specialties that take part in the decision-making and treatment planning are represented during an MDT meeting, including a surgeon, medical oncologist, radiation oncologist, pathologist, radiologist, and specialised nurses. MDT meetings are conducted routinely, preferably weekly, at expert centers. Ideally, each cancer patients is discussed pre- and postoperatively in an MDT-meeting. Reviews and guidelines have been published regarding the composition, optimization, and practical barriers of a successful MDT and factors influencing the decisions made by an MDT. ${ }^{18-20}$ The most important task of the MDT centers around the preoperative staging and treatment strategy of a newly diagnosed rectal cancer patient. Involvement of the MRF is associated with an increasing incidence of local recurrence, distant metastases, and poor survival, and is the most important predictor in oncological outcome. ${ }^{21,22}$ Preoperative MRI staging in combination with multidisciplinary team assessment has been shown to reduce the incidence of positive circumferential margins. ${ }^{23}$ More recent articles demonstrated a significant improvement in oncological outcomes due to MDT meetings, with decreased recurrence rates and increased survival. ${ }^{24,25}$ The factors influenced by the MDT meetings and contributing to this improved oncological outcome include an increased number of patients undergoing an MRI in preoperative staging, ${ }^{26}$ who received neoadjuvant treatment, ${ }^{24,26}$ who underwent surgery for metastatic disease, ${ }^{27}$ and who receive adjuvant chemotherapy. ${ }^{25}$ There are some limitations to these studies, and their design and improvement in perioperative patient care, fast-track surgery, surgical techniques, and the introduction of multimodality treatment without routine MDT meetings may have contributed to their positive results.

\section{Centralization}

The centralization of cancer care is a recent advancement in colorectal cancer treatment, influencing treatment outcomes for advanced cases. As described previously, the management and treatment of this heterogeneous group of patients with tumor growth beyond traditional TME planes is complex and requires experience in all fields of cancer care. With involvement of surrounding organs, locally advanced and recurrent cases more often require multivisceral resections in which enbloc resection of gynaecological or urological organs are performed. The incidence of these tumors in a general hospital is low and this makes gaining experience is these situations difficult. Centralization of the care of these patients will likely lead to increased experience among varying disciplines at expert centers, positively influencing 
oncological outcomes, ${ }^{28}$ due to improved rates of radical resections and lowered surgical morbidity and mortality rates ${ }^{29}$ secondary to optimized postoperative care.

Some centers may also consider intraoperative radiotherapy (IORT) as a supplemental treatment in extended T4 cases and locally recurrent cases. A high-dose electron or photon beam irradiation boost using a linear accelerator or other devices in the operating room can be delivered to a specific, small, high-risk area with threatened or positive margins, while minimizing risk of damage to surrounding organs. In these selected cases, the infield recurrence rate may be reduced by $50 \%{ }^{30,31}$ Current studies on IORT include various tumors and indications for IORT due to the differences in selection criteria and expertise between expert centers. Future studies will have to further define selection criteria for high-risk patients that will benefit from IORT.

\section{Registration and auditing}

As mentioned previously, outcomes of colorectal cancer treatment differ not only widely among countries, but also within countries among hospitals, and even within hospitals among individual surgeons. ${ }^{14,32-34}$ Given the importance of each entity within the treatment of colorectal cancer, the quality of all modalities are equally important in the pursuit of improvement in treatment outcomes. If the chain of preoperative radiological staging, neoadjuvant treatment, surgical treatment and pathologic analysis and adjuvant treatment is excellent, it is possible to unravel the varying prognostic variables that apply to an individual patient. Using this approach, the biology of the tumor, quality of surgery, and the effect of neoadjuvant treatment can be assessed. Furthermore, high-risk patients are identified earlier in the treatment course and treatment can be adjusted accordingly. Registering data on these different variables will enable the identification of deficiencies in evaluation and therapy. It will allow its use to raise the quality of the entire process, and to improve outcomes and reduce the burden of treatment costs for avoidably poor results. ${ }^{35}$

In parts of Europe, registration has been initiated on an individual hospital level. These individual registries were the basis for a larger national registry. ${ }^{36}$ In Norway, Sweden, Denmark, the UK, and the Netherlands, mandatory registration has led to an almost $100 \%$ coverage of these respective populations. These registries, however, collect data on multiple malignancies, and therefore sometimes lack data specifics, for example on the details of pre-operative evaluation and postoperative complications. In 1993, Norway initiated the first audit on colorectal cancer with their Norwegian ColoRectal Cancer Project. ${ }^{37}$ After 4 years of this process, a decrease in local recurrence rates from 28 to $7 \%$ was achieved. 
In 2009, a nationwide audit was initiated in the Netherlands by the Association of Surgeons of the Netherlands. This audit (the Dutch Surgical Colorectal Audit (DSCA)) was developed to monitor, evaluate, and improve colorectal cancer care in the Netherlands. ${ }^{38}$ Within 2 years, the full participation of Dutch hospitals was realized as this audit became a national performance and quality indicator. In this data collection process, each hospital had to appoint one surgeon to be responsible for the hospital registry. In this audit, up to 179 variables are logged, ranging from basic patient data, comorbidities, diagnostic workup, neoadjuvant treatment (if applicable), surgery, pathology, and postoperative complications, as well as the administration of adjuvant treatment. Data collected in the DSCA are compared to and validated with the National Cancer Registry, which routinely collects all newly diagnosed malignancies with a data completeness rate estimated to be at least $95 \% .{ }^{39}$

In the period 2009-2011, 26,511 patients from all 92 hospitals undergoing surgical resection for colorectal cancer were included in the registry. Similar to the NCR registration database, the registration rate improved over time, expanding from an initial 80 to $95 \%$ of patients being registered in 2011. In the most recent analysis of the DSCA data by van Leersum et al., an increase in colorectal cancer patients discussed in preoperative MDT meetings was determined, increasing from 46 to $68 \%$ in colon cancer cases and 80 to $96 \%$ in rectal cancer patients. ${ }^{38}$ Furthermore, an increase in the use of pre-operative $\mathrm{MRI}$ as well as a major increase in pathologists reporting CRM margins (increasing from 48 to $80 \%$ ) were found. A decrease in post-operative complications was seen for both colon and rectal cancer patients, and over the study period, more radical resections were achieved. With the use of nationwide registries on specific cancer care, it is possible to determine whether hospitals treat patients according to national guidelines and if there are differences in the treatment between hospitals. Outcomes and quality of treatment between various high volume hospitals for specific cancer care can easily be compared. Furthermore, surgeons and hospitals can improve their quality of treatment by learning from their own results and comparing their outcomes with other hospitals.

To compare the treatment and outcomes of cancer within Europe, the EUROCARE project was introduced in 1990. In this project, 83 population-based cancer registries from 23 countries across Europe supplied data on the incidence and follow-up of cancer patients diagnosed at any age in various time periods from 1978 to 2002. In the analysis containing colorectal cancer data, large differences in outcomes were seen across Europe. ${ }^{14}$ In addition, EUROCARE reports showed that survival was improving but intercountry variation persisted over the years. ${ }^{40} \mathrm{~A}$ limitation on the overall use of the EUROCARE database is the wide variation in coverage of populations between the European countries, varying from $1 \%$ in Germany to $100 \%$ coverage in the UK. This large variation makes it difficult to compare results between countries. 
The United States have their own cancer registry with the Surveillance, Epidemiology, and End Results (SEER) Program of the National Cancer Institute. In the most recent study using the SEER database, endoscopic, and local excision techniques were compared to surgery for early colorectal cancer, including T1-T2 stage disease. For early rectal cancer (in situ and T1), these investigator found equivalent results in outcomes as compared to major surgery, but inferior outcomes were seen for $\mathrm{T} 1 / 2$ colon cancer and T2 rectal cancers. ${ }^{41}$ In another study focusing on survival over time in patients with stage IV colorectal cancer, an improved survival was seen in patients $<70$ years with stage IV colorectal cancer, although not in older patients with stage IV disease. ${ }^{42}$ The strength of the SEER database lies in large patient samples, but it also has its limitations, given that vital information on treatment, pathological information, and complications are not registered.

The driving force behind the European international multidisciplinary registry is the idea that the quality of treatment is not measured on individual bases or treatment modality, but instead involves the entire course of treatment from diagnosis to completion. In order to measure differences between countries and set consensus on the best possible treatment, the European CanCer Organisation (ECCO) initiated EURECCA, an international, multidisciplinary outcome-based quality improvement program. The goal of this registration is to generate the best possible care for rectal cancer and to meet the demands for transparency. ${ }^{36}$ EURECCA is a European audit using data of the nationwide colorectal audit registries. Until mid-2011, 418,500 patients from nine different registries were included in EURECCA. After comparing the datasets, 45 data items, including the most vital variables, could be identified and were included in the core dataset. ${ }^{43}$ These data items are shown in Table 9.1.

Another promising multidisciplinary initiative in Europe is the initiation of European consensus meetings. These meetings were held in order to achieve international guidelines in Europe. In the third and most recent European Consensus meeting in December 2012 in Perugia, Italy, a multidisciplinary expert panel involved in colorectal cancer care was convened in order to establish a firm basis of treatment strategies for health professionals in the field. This expert panel consisted of representatives from multiple European Scientific Organisations (i.e. ECCO, ESTRO and ESSO) (44). The aim of this meeting was to outline the core quality treatment strategies for colon and rectal cancer, using the Delphi method to reach consensus between the experts. ${ }^{45}$ The consensus meeting achieved large consensus in $84 \%$ of the sentences provided, meaning that $95 \%$ of the experts agreed on these statements. Implementation of these guidelines in day-to-day practice is the next challenge. In the future, this could be measured by the EURECCA program. 
Although it is widely accepted that advanced cases should be treated in expert centers, treatment regimens differ widely. The "Beyond TME Collaborative" (BTC) is one of the first worldwide initiatives to set guidelines for advanced cases, attempting to improve the overall quality of rectal cancer care and provide a clear framework for the assessment and management of these complex patients. ${ }^{11}$ The Beyond TME group compromises representatives from specialist centers offering treatment for advanced pelvic cancer in the USA, Canada, Australia, New Zealand, China, and Europe. Recently, the BTC developed a consensus statement providing guidance for the management of patients with locally advanced and locally recurrent rectal cancer beyond the TME planes. During the two consensus meetings held between September 2011 and March 2012, consensus was achieved for pre-operative assessment, neo-adjuvant therapy, surgery, and much more. Multiple algorithms on diagnosis, assessment of resectability, and the use of neo-adjuvant and adjuvant treatment were also created. The BTC concluded that these patients should be recognized as high-risk patients and should be referred to specialized expert centers. This initiative to share expertise, rather than be the best, is a modern mindset in the treatment of colorectal cancer.

Although the importance of registries in cancer care is recognized, it is facing an uncertain future, as registries depend on funding and are affected by its constraints. Furthermore, implementation of legislation on informed consent and registration of patient-related data can be a restricting factor in data collection. Studying differences between countries with the use of registries is only possible if the same variables are measured and registered according to internationally accepted techniques. Easy exchange of data between countries, for survival and treatment analysis, is required in order to set international guidelines. However, one could also argue that if in a given country no such thing as a comprehensive registry exists, these countries should not wait until a registry is installed. Instead, they should implement guidelines on the basis of international consensus statements, explicitly stating why deviations from this consensus apply for their specific situation. 
Table 9.1 Data items scored by the European Colorectal Cancer Audit Registries (EURECCA).

\begin{tabular}{|c|c|c|}
\hline & EURECCA core dataset & Input variable \\
\hline \multirow[t]{10}{*}{ Patient administrative data } & Patient number & \\
\hline & Cancer type & Rectal / colon \\
\hline & Neo-adjuvant therapy & None / RT / CT / RCT* \\
\hline & Adjuvant therapy & None / RT / CT / RCT \\
\hline & Gender & \\
\hline & Patient Name & \\
\hline & Hospital & \\
\hline & Date of birth & \\
\hline & Radiotherapy & Type (preoperative/postoperative) \\
\hline & & Duration (short-/long-course) \\
\hline \multirow[t]{6}{*}{ Patient preoperative staging } & ASA Endoscopy & $\mathrm{Y} / \mathrm{N} * *$ \\
\hline & & Lowe limit from anal verge \\
\hline & CT scan & $\mathrm{Y} / \mathrm{N}$ \\
\hline & Ultrasound rectal & $\mathrm{Y} / \mathrm{N}$ \\
\hline & MRI scan & $\mathrm{Y} / \mathrm{N}$ \\
\hline & & cTstage \\
\hline \multirow[t]{3}{*}{ Preoperative final staging } & cT-stage & \\
\hline & cN-stage & \\
\hline & cM-stage & \\
\hline \multirow[t]{2}{*}{ Neo-adjuvant treatment } & Preoperative chemotherapy & Type/technique/dose \\
\hline & Preoperative radiotherapy & \\
\hline \multirow[t]{7}{*}{ Operation } & Operation & $\mathrm{Y} / \mathrm{N}$ \\
\hline & & Date \\
\hline & Main procedure & ICD 10 diagnosis or translatable into \\
\hline & Surgery stoma & $\begin{array}{l}\text { None, decompressive, permanent, loop, end- } \\
\text { type }\end{array}$ \\
\hline & Surgical access for resection & $\begin{array}{l}\text { Laparotomy/laparoscopy/converted } \\
\text { laparoscopy/local excision }\end{array}$ \\
\hline & Surgery anastomosis & \\
\hline & Resection of adjacent organs & \\
\hline Postoperative complications & Anastomotic leakage & \\
\hline Postoperative staging pathology & Primary diagnosis & ICD 10 \\
\hline \multirow[t]{11}{*}{ Final diagnosis } & Distant metastasis & \\
\hline & Histology & \\
\hline & pT & \\
\hline & $\mathrm{pN}$ & \\
\hline & $\mathrm{pM}$ & \\
\hline & Tumor stage & \\
\hline & Lymphnodes in total & \\
\hline & Lymphnodes with metastasis & \\
\hline & CRM & \\
\hline & Radicality operation & $\mathrm{R} 0 / \mathrm{R} 1 / \mathrm{R} 2$ \\
\hline & Location distant metastasis & $\begin{array}{l}\text { Liver/lung/peritoneum/omentum/ovary/lymph } \\
\text { node }\end{array}$ \\
\hline Adjuvant treatment & $\mathrm{RT} / \mathrm{CT} / \mathrm{RCT}$ & Type technique dose \\
\hline \multirow[t]{2}{*}{ Follow-up } & Date of death & \\
\hline & Dead/alive & \\
\hline
\end{tabular}

$* \mathrm{RT}=$ radiotherapy; $\mathrm{CT}=$ chemotherapy; $\mathrm{RCT}=$ chemoradiation. ${ }^{*} * \mathrm{Y}=\mathrm{Yes} ; \mathrm{N}=$ No. 


\section{Conclusion}

For most patients with tumors limited to the TME planes, referral to specialized cancer centers is not necessarily required. In addition, referral of these patients will result in an overload of patients to these centers, resulting in longer waiting periods and fewer opportunities to treat advanced cases. Referral of advanced cases to specialist centers will result in improved rates of radical resections in their own hospitals and will improve overall rates of non-curative resection. Recently published data on sharing expertise and referral of advanced cases to specialist centers have shown good results for all hospitals in the region. ${ }^{46}$ With the use of regional data in the southern Netherlands, with a total of 5,529 patients, no differences in the proportions of anastomotic leakage, 30-daymortality rates, and hazard risks for death exist between high- and low-volume hospitals for both colon and rectal cancer patients. Although these high-volume hospitals treat more advanced cases, there is good quality of care, and R0 resection rates for the whole region have increased due to adequate patient distribution.

In the present era, patients should no longer be at risk of misdiagnosis, under- or over staging, or receiving inappropriate treatment. On a patient scale, the patient will benefit most if he is treated by the physician with the best expertise in colorectal treatment. Given this fact, this physician has to be expertly prepared in order to treat patients, but on an individual basis he is only able to treat a minority of all patients. On a population-based and international level, patients may benefit more if there is not a small group of experts, but instead if each physician treats according to the guidelines formed by expert consensus panels. This allows patient care to be independent of the region or country in which a given patient may live. A shift in mindset by physicians and experts has to occur in order to achieve the best possible treatment for each patient. We have to share our expertise in colorectal cancer. Sharing such expertise will not result in some kind of competition, i.e., "who treats the most patients" or "who has the lowest number of irradical resections". Rather, it gives others the possibility to use and benefit from expert opinions. Initiatives such as the European consensus meeting and the Beyond TME collaborative were international and worldwide guidelines created to share these thoughts and improve the overall quality of care, nationally and internationally.

We also have to be aware that standardizing treatment is not necessarily the ultimate solution to optimized cancer care. As patients differ widely in pre-treatment performance status, disease stage, and many other important clinical-demographical factors, physicians can do more harm rather than good by blindly implementing standardized treatment. With the use of guidelines it is possible to treat the majority of the patients, but physicians still have to be aware of patients harbouring comorbidities and their ability to tolerate such treatments. In this era of standardization, registration, 
and auditing, there has to be room for a patient-tailored treatment, and guidelines should not lead to a one-size-fits-all treatment.

In the last decade, rectal cancer treatment has evolved from a surgery-alone treatment to a multimodality and multidisciplinary treatment. All modalities have contributed to improved survival. Using international guidelines and guidelines from consensus meetings, we now know which treatment strategy is needed to achieve optimal oncological outcomes. In some cases, a very intensive treatment is needed in order to achieve this result. This intensive treatment can have a tremendous effect on a patient's health-related quality of life (HRQL). In some cases, patients have other expectations for life, and oncological outcomes are less important. In shared decisionmaking, doctors and patients actively discuss and decide on therapeutic interventions, in order to reach a common goal instead of clinician-driven goal based on oncological outcome. This type of treatment is increasingly important, especially in the elderly patient in whom expectations of HRQL rather than oncological outcome play an important role in the decision. Ultimately, effectively involving patients in decisions about their treatment will reduce the risk of doing more harm than good.

Despite the above, and that there is a profound interest among patients and the media to find the 'best' doctors for the treatment of rectal cancer, it is much better to elevate the skills of complete teams, which are fundamentally necessary to provide optimal care. With the use of registration, auditing, and the introduction and support for international guidelines, there is no need to create a scale between countries, hospitals, or physicians. In the end, there is no need for more experts or authorities, but instead it is preferable to share our expertise and effort to make sure that every patient, regardless of origin, is treated with the best cancer care. 


\section{References}

1. Heald RJ, Ryall RD. Recurrence and survival after total mesorectal excision for rectal cancer. Lancet 1986;1:1479-82.

2. Quirke P, Durdey P, Dixon MF, Williams NS. Local recurrence of rectal adenocarcinoma due to inadequate surgical resection. Histopathological study of lateral tumour spread and surgical excision. Lancet 1986;2: 996-9.

3. Kennelly RP, Rogers AC, Winter DC. Multicentre study of circumferential margin positivity and outcomes following abdominoperineal excision for rectal cancer. Br J Surg 2013;100:160-6.

4. Nagtegaal ID, Quirke P. What is the role for the circumferential margin in the modern treatment of rectal cancer? J Clin Oncol 2008;26:303-12.

5. Extramural depth of tumor invasion at thin-section MR in patients with rectal cancer: Results of the MERCURY study. Radiology 2007;243:132-9.

6. Taylor FG, Quirke P, Heald RJ, et al. One millimetre is the safe cut-off for magnetic resonance imaging prediction of surgical margin status in rectal cancer. Br J Surg 2011;98:872-9.

7. Doornebosch PG, Bronkhorst PJ, Hop WC, Bode WA, Sing AK, de Graaf EJ. The role of endorectal ultrasound in therapeutic decision-making for local vs. transabdominal resection of rectal tumors. Dis Colon Rectum 2008;51:38-42.

8. van Gijn W, Marijnen CA, Nagtegaal ID, et al. Preoperative radiotherapy combined with total mesorectal excision for resectable rectal cancer: 12-year follow-up of the multicentre, randomised controlled TME trial. Lancet Oncol 2011;12:575-82.

9. Sauer R, Liersch T, Merkel S, et al. Preoperative versus postoperative chemoradiotherapy for locally advanced rectal cancer: results of the German CAO/ARO/AIO-94 randomized phase III trial after a median follow-up of 11 years. J Clin Oncol 2012;30:1926-33.

10. Bosset JF, Calais G, Mineur L, et al. Enhanced tumorocidal effect of chemotherapy with preoperative radiotherapy for rectal cancer: preliminary results--EORTC 22921. J Clin Oncol 2005;23:5620-7.

11. Consensus statement on the multidisciplinary management of patients with recurrent and primary rectal cancer beyond total mesorectal excision planes. Br J Surg 2013;100:E1-33.

12. de Campos-Lobato LF, Geisler DP, da Luz MA, Stocchi L, Dietz D, Kalady MF. Neoadjuvant therapy for rectal cancer: the impact of longer interval between chemoradiation and surgery. J Gastrointest Surg 2011;15:444-50.

13. Sloothaak DA, Geijsen DE, van Leersum NJ, et al. Optimal time interval between neoadjuvant chemoradiotherapy and surgery for rectal cancer. Br J Surg 2013;100:933-9.

14. Brenner H, Bouvier AM, Foschi R, et al. Progress in colorectal cancer survival in Europe from the late 1980s to the early 21st century: the EUROCARE study. Int J Cancer 2012;131:1649-58.

15. Ferlay J, Steliarova-Foucher E, Lortet-Tieulent J, et al. Cancer incidence and mortality patterns in Europe: estimates for 40 countries in 2012. Eur J Cancer 2013;49:1374-403.

16. Augestad KM, Lindsetmo RO, Stulberg JJ, et al. System-based factors influencing intraoperative decision-making in rectal cancer by surgeons: an international assessment. Colorectal Dis 2012;14: e679-88.

17. Calman K, Hine D. A policy framework for commisioning cancer services. Chief Medical Officers' Expert Advisory Group on Cancers. (Department of Health and Welsh Office, 1995). 2013.

18. Ruhstaller T, Roe H, Thurlimann B, Nicoll JJ. The Multidisciplinary Meeting: An Indispensable Aid to Communication Between Different Specialities. Eur J Cancer 2006; 42(15): 2459-62.

19. Fleissig A, Jenkins V, Catt S, Fallowfield L. Multidisciplinary teams in cancer care: are they effective in the UK? Lancet Oncol 2006;7:935-43.

20. Lamb BW, Brown KF, Nagpal K, Vincent C, Green JS, Sevdalis N. Quality of care management decisions by multidisciplinary cancer teams: a systematic review. Ann Surg Oncol 2011;18:2116-25.

21. Glimelius B, Beets-Tan R, Blomqvist L, et al. Mesorectal fascia instead of circumferential resection margin in preoperative staging of rectal cancer. J Clin Oncol 2011;29:2142-3.

22. Quirke P, Steele R, Monson J, et al. Effect of the plane of surgery achieved on local recurrence in patients with operable rectal cancer: a prospective study using data from the MRC CRO7 and NCIC-CTG CO16 randomised clinical trial. Lancet 2009;373:821-8. 
23. Burton S, Brown G, Daniels IR, Norman AR, Mason B, Cunningham D. MRI directed multidisciplinary team preoperative treatment strategy: the way to eliminate positive circumferential margins? $\mathrm{Br} J$ Cancer 2006;94:351-7.

24. Palmer G, Martling A, Cedermark B, Holm T. Preoperative tumour staging with multidisciplinary team assessment improves the outcome in locally advanced primary rectal cancer. Colorectal Dis 2011;13: 1361-9.

25. MacDermid E, Hooton G, MacDonald M, et al. Improving patient survival with the colorectal cancer multi-disciplinary team. Colorectal Dis 2009;11:291-5.

26. Augestad KM, Lindsetmo RO, Stulberg J, et al. International preoperative rectal cancer management: staging, neoadjuvant treatment, and impact of multidisciplinary teams. World J Surg 2010;34:2689-700.

27. Segelman J, Singnomklao T, Hellborg H, Martling A. Differences in multidisciplinary team assessment and treatment between patients with stage IV colon and rectal cancer. Colorectal Dis 2009;11:768-74.

28. McArdle CS, Hole DJ. Influence of volume and specialization on survival following surgery for colorectal cancer. Br J Surg 2004;91:610-7.

29. Borowski DW, Kelly SB, Bradburn DM, Wilson RG, Gunn A, Ratcliffe AA. Impact of surgeon volume and specialization on short-term outcomes in colorectal cancer surgery. Br J Surg 2007;94:880-9.

30. Mirnezami R, Chang GJ, Das P, et al. Intraoperative radiotherapy in colorectal cancer: systematic review and meta-analysis of techniques, long-term outcomes, and complications. Surg Oncol 2013;22:22-35.

31. Kusters M, Valentini V, Calvo FA, et al. Results of European pooled analysis of IORT-containing multimodality treatment for locally advanced rectal cancer: adjuvant chemotherapy prevents local recurrence rather than distant metastases. Ann Oncol 2010;21:1279-84.

32. Birbeck KF, Macklin CP, Tiffin NJ, et al. Rates of circumferential resection margin involvement vary between surgeons and predict outcomes in rectal cancer surgery. Ann Surg 2002;235:449-57.

33. Porter GA, Soskolne CL, Yakimets WW, Newman SC. Surgeon-related factors and outcome in rectal cancer. Ann Surg 1998;227:157-67.

34. Richardson DP, Porter GA, Johnson PM. Surgeon knowledge contributes to the relationship between surgeon volume and patient outcomes in rectal cancer. Ann Surg 2013;257:295-301.

35. Pahlman L, Bohe M, Cedermark B, et al. The Swedish rectal cancer registry. Br J Surg 2007;94:1285-92.

36. van Gijn W, van de Velde CJ. Improving quality of cancer care through surgical audit. Eur J Surg Oncol 2010;36 Suppl 1:S23-S26.

37. Wibe A, Moller B, Norstein J, et al. A national strategic change in treatment policy for rectal cancer-implementation of total mesorectal excision as routine treatment in Norway. A national audit. Dis Colon Rectum 2002;45:857-66.

38. van Leersum NJ, Snijders HS, Henneman D, et al. The Dutch surgical colorectal audit. Eur J Surg Oncol 2013.

39. Schouten LJ, Jager JJ, van den Brandt PA. Quality of cancer registry data: a comparison of data provided by clinicians with those of registration personnel. Br J Cancer 1993;68:974-7.

40. Gatta G, Capocaccia R, Sant M, et al. Understanding variations in survival for colorectal cancer in Europe: a EUROCARE high resolution study. Gut 2000;47:533-8.

41. Bhangu A, Brown G, Nicholls RJ, Wong J, Darzi A, Tekkis P. Survival outcome of local excision versus radical resection of colon or rectal carcinoma: a surveillance, epidemiology, and end results (SEER) population-based study. Ann Surg 2013;258:563-71.

42. Golan T, Urban D, Berger R, Lawrence YR. Changing prognosis of metastatic colorectal adenocarcinoma: differential improvement by age and tumor location. Cancer 2013;119:3084-91.

43. van Gijn W, van den Broek CB, Mroczkowski $P$, et al. The EURECCA project: data items scored by European colorectal cancer audit registries. Eur J Surg Oncol 2012;38:467-71.

44. van de Velde CJ, Aristei C, Boelens PG, et al. EURECCA colorectal: multidisciplinary mission statement on better care for patients with colon and rectal cancer in Europe. Eur J Cancer 2013;49:2784-90.

45. Rowe G, Wright G. The Delphi technique as a forecasting tool: issues and analysis. Int J Forecast. 353375, 1999.

46. van Erning FN, van Steenbergen LN, van den Broek WT, Rutten HJ, Lemmens VE. No difference between lowest and highest volume hospitals in outcome after colorectal cancer surgery in the southern Netherlands. Eur J Surg Oncol 2013;39:1199-206. 

perspectives

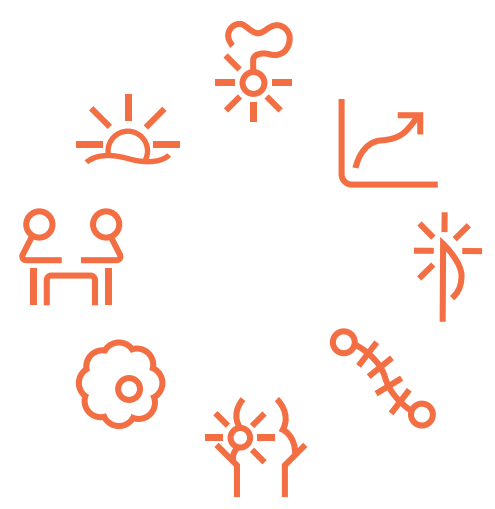


Chapter 10 
On a worldwide scale, colorectal cancer is one of the leading causes death by cancer affecting millions of people every year. One third of colorectal cancer cases concerns the rectum. In more than two third of the cases rectal cancer is localised to the pelvis without detectable distant metastasis. Although neo-adjuvant treatment can effectively downsize locally advanced tumours and reduce locally recurrent rates, surgery is still the cornerstone for cure in the treatment for rectal cancer. On the other hand, surgery is just a part in the multidisciplinary treatment a patient is given. Only with maximum effort of all disciplines involved in treatment chain of rectal cancer the best possible outcome can be achieved. For example, if the pathology is excellent, it will unravel the different prognostic variables which apply to an individual patient. Not only the biology of the tumour can be determinative but also the quality of surgery and the effect of neo-adjuvant treatment. The first steps in integration of optimal imaging, treatment modalities and pathology are already made in several countries. The next step should be to optimise treatment for the individual patient, who is not only interested in the oncological outcome but also in functional results and subsequent quality of life.

\section{Age related differences in rectal cancer}

In 2015 rectal cancer occurred in The Netherlands in 4342. With increasing life expectancy, it can be assumed that there will be more and more patients affected by rectal cancer. ${ }^{1}$ This is also highlighted in Figure 101 in which the increasing incidence of rectal cancer is shown from 1990 till 2015. Furthermore, it is shown that three out of four patients diagnosed with rectal cancer is 60 years or older.

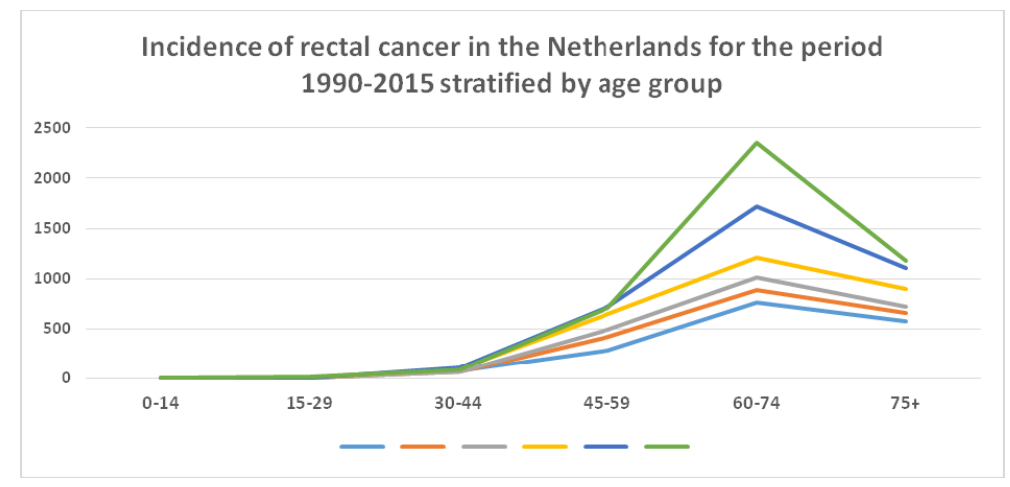

Figure 10.1 Incidence of rectal cancer in the Netherlands for the period 1990-2015 stratified by age group. Data retrieved from cijfersoverkanker.nl 
An increasing incidence of patients diagnosed with rectal cancer at the age of 40 years or younger is also observed. ${ }^{2,3}$ Young patients with rectal cancer more often present with advanced stage of disease and unfavourable tumour morphology compared with middle aged or elderly patients. ${ }^{4-7}$ Studies focussing on survival in young rectal cancer patients show conflicting results ranging from unfavourable to better survival compared to older patients. ${ }^{4,-9}$ The assumption that younger patients with rectal cancer have poorer survival is mainly based on studies performed prior or during the introduction of TME surgery and neo-adjuvant treatment schemes. ${ }^{9,10}$ To have knowledge of age related differences in rectal cancer patients two studies were conducted. First in Chapter 3, differences in tumour morphology, treatment and survival in patients aged $\leq 40$ years were compared to middle aged patients (41-70) years. These two age groups were chosen because life expectancy is good in both groups and less confounded by other variables, such as co-morbidities or age dependent physiological decline, unlike in the oldest group (70 years or older). For this study population-based data from the Netherlands Cancer Registry (NCR) was used and more than 37.000 patients were included. Young patients presented with more advanced stage of disease and had more unfavourable tumor characteristics compared with middle aged-aged patients. Despite these characteristics survival rates were equal and in multivariate analyses young age remained a prognostic factor for better survival. Another important age related difference is that elderly patients are at risk of receiving a non-curative treatment, which negatively affects survival. ${ }^{11,12}$ In a Dutch study only $62 \%$ of the elderly patients ( $\geq 75$ years) with a stage I-III rectal cancer were treated according to the guidelines, compared to $87 \%$ of the patients aged $<75$ years. ${ }^{13}$ Age was the primary reason for withholding curative treatment in this study. However, this decision should not be based on age alone, as the patients' chronical age is a poor reflection of their actual performance status. ${ }^{14,15}$ Particularly in advanced cases with increased risk of morbidity and mortality elderly are at risk of not receiving curative treatment. Little is known on how elderly with locally advanced (LRRC) and locally recurrent (LRRC) rectal cancer endure curative treatment with major rectal surgery. In Chapter 4 the morbidity and mortality was described on patients treated with curative intent for cT4 rectal cancer (cT4RC) and LRRC with a special focus on elderly patients.

Extensive multimodality treatment for patients with LARC or LRRC is associated with a high morbidity rate. In the current study, $56 \%$ of the cT4RC $<75$ years and $69 \%$ of the patients $\geq 75$ years had at least one complication. After treatment for LRRC $75 \%$ of the patients $<75$ years and $96 \%$ of the patients $\geq 75$ years experienced at least one complication. Despite the high morbidity, in hospital mortality was only $2.5 \%$ in all patients. However, it is known that particularly in elderly, 30-day mortality rate is a high underestimation of the one year mortality rate. ${ }^{16,17}$ This was also revealed in the current study with a one-year mortality being 2.5 and 3.5 times higher in CT4RC and LRRC elderly patients respectively, compared to the 30-day mortality rate. In younger 
cT4RC patients this delayed "postoperative" mortality was not observed. The multivariable binary regression analyses on predictive factors for one-year mortality revealed age $\geq 75$ years (OR 3.83) as a significant predictor for increased one-year mortality. Other significant factors for increased risk for one-year mortality were a total exenteration and grade IV complications. In LRRC, underestimation of the one-year survival rate compared to the 30-day mortality rate was also observed but this was not age related and multivariable analyses only revealed a significant effect of grade IV complications on one-year mortality. In LRRC, there is probably a mixed effect of both the more aggressive biological behavior of the tumor and the morbidity of the treatment on survival.

\section{Impact of rectal cancer treatment on health-related quality of life}

The current approach in the treatment of rectal cancer requires the obligation to have knowledge on the influence of treatment on patients' health related quality of life (HRQL). The patient needs to be well informed about the choices that can be made regarding his/her treatment and they are allowed to have their own say in the matter. In order to have more knowledge about quality of life after surgery, the effect of tumour stage and subsequent treatment, extent of surgery and influence of a permanent stoma on HRQL were investigated in this thesis.

In Chapter $\mathbf{5}$ the influence of disease stage and subsequent treatment on the HRQL of rectal cancer patients were investigated. Patients were divided into three groups: those patients with a primary cT1-T3 tumour without pathological lymph nodes and involved margin (NAD-group), patients LARC and patients with a LRRC. Patients with LRRC reported a lower HRQL compared to patients with LARC and NAD. There were differences in Future Perspective and Physical, Social and Sexual Function scales compared to healthy individuals and both NAD and LARC patients. All three groups reported low scores on sexual functioning compared to healthy individuals while only a few patients reported to use aids to improve this functioning. A drop in sexual activity was seen in all patients. Fewer than $50 \%$ of all men were sexually active and only $35 \%$ of women were sexually active after treatment.

$\mathrm{HRQL}$ is the lowest after treatment for recurrent rectal cancer compared to primary rectal cancer. Furthermore, we observed no large differences in HRQL between patients with NAD and LARC. In LARC the tumour is invading the mesorectal fascia or is even penetrating into surrounding pelvic structures. These tumours are treated with other neo-adjuvant treatment modalities and need often a more aggressive surgical approach in order to achieve clear resection margins. Extended resections may result in loss of autonomic nerves, other pelvic supporting structures (eg, sacrum or pelvic floor muscles) or organs (eg, bladder, genital organs or ureters). The possible loss of 
autonomic pelvic nerve function and the mutilating effects of these extended resection can have a tremendous effect on the patients HRQL. To investigate the effect of extended (multivisceral) resections on HRQL a study was performed in only primary rectal cancer patients (Chapter 6). Patients treated with standard TME surgery were compared to patients treated with extended surgery outside the TME plane. To investigate the age related differences we further stratified these groups in elderly $(\geq 70$ years) and younger patients ( $<70$ years). In males the vesicles $(65 \%)$ and prostate $(>40 \%)$ were most often partially or complete resected in the extended resections. The vaginal dorsal wall (>45\%), ovaries (65\%) and uterus (53\%) were the most often resected organs in women. Other resected organs include the sacrum (S3/S4 or Coccyx), the bladder (partially or complete with Bricker reconstruction), ureter (with reconstruction) and additional bowel resections. Patients undergoing extended resections reported an equally HRQL compared to TME surgery patient with the exception of body image which was significantly lower in patients $<70$ years after extended surgery compared to all other treatment groups. Not surprisingly, sexual function was lower in the elderly patient group compared to younger patients, whereas older patients reported the lowest sexual function after extended surgery. We expected to find large differences on the sexuality and micturition subscales between TME and extended surgery because of the increased risk of pelvic nerve damage. However, no significant differences were observed on sexual function and male sexual dysfunction subscale between TME and extended surgery in patients $<70$ years. Although on specific item level, male patients undergoing TME surgery reported less frequent erectile and ejaculatory problems compared to extended surgery group. In women, we observed no significant differences between all groups. This could be due to the low number of sexually active women after treatment (only 25\%). Although large differences could not be observed in this study, we believe that on an individual patient level these differences do exist and one should be aware of the influence of extended resections on the individual patient.

In Chapter 7 the impact of a permanent stoma on the patients' HRQL was investigated. The decision between a LAR with a restored bowel continuity and a LAR/APR with a permanent stoma in patients with distal rectum cancer may be difficult. This is especially true for elderly patients in whom the assumed benefits of the avoidance of a stoma should be outweighed against the potentially life threatening complications such as anastomotic leakage. When anastomotic leakage occurs in the elderly, the subsequent mortality rate rises to $57 \% .{ }^{18}$ Knowledge of the impact of a stoma on the HRQL may help to determine a treatment strategy for elderly or frail patients that is both safe and preserves a good HRQL. For this study data from the Eindhoven Cancer Registry (ECR) were used, which covered a population of 2.4 million inhabitants in the Southern part of the Netherlands. Patients with low rectal cancer $(\leq 10 \mathrm{~cm}$ from anal verge) who underwent surgery in four regional hospitals were selected. Patients with a permanent stoma were compared to those without a stoma and were further stratified 
by age ( $<70$ and $\geq 70$ years). These groups were also compared to a normative population. Interestingly, the presence of a stoma had only a minor influence on the HRQL irrespective of age. Older patients with a stoma have comparable HRQL to older patients without a stoma or the normative population. Age itself seemed to be of more impact since older patients experienced worse physical function when compared to younger patients irrespective of the presence of a stoma. Keeping in mind the severe impact that post-operative complications, in particular anastomotic leakage, can have on clinical recovery, a permanent stoma is feasible for elderly and frail patients with a low carcinoma.

In both Chapter $\mathbf{5}$ and Chapter $\mathbf{6}$ a comparison of the selected treatments groups with a healthy (normative) population was performed. In conclusion, HRQL of primary rectal cancer patients was almost similar to that of a normative population despite the extensive treatment that these patients often have undergone. However, as addressed in Chapter 5-7 rectal cancer treatment negatively affects sexual functioning and sexual activity. These results were not unexpected: the extent of surgery and the natural decline of sexual activity with increasing age may have caused the steepest decline in sexual activity. However, in other studies sexual problems were also reported with an incidence of sexual dysfunction of $23 \%-69 \%$ in men and a decline of $30-40 \%$ of previously sexually active patients reporting inactivity following treatment. ${ }^{19}$ Interestingly, only a minority of the patients reported the use of aids in order to improve their sexual functioning. The negative impact of treatment on sexual function as found in this thesis calls for further attention and awareness to alleviate this problem in sexually active patients. For optimal consultation, sexual dysfunction due to surgery should be actively discussed during a patients visit.

Although we observed no major influence of neither stage of disease, extent of surgery, or presence of a stoma on HRQL, we should keep in mind that HRQL and sexual function are both multifactorial adaptive processes. Sexual function and HRQL are not only influenced by treatment related factors but also by preoperative sexual activity and psychosocial functioning. This makes it difficult to detangle the influence of one specific treatment modality on HRQL and sexuality, not only in these studies but in colorectal cancer research in general. The lack of differences can also be explained by a shift in expectations (e.g., response shift). A response shift is a phenomenon whereby patients change their internal standards as an adaptation to the limitations caused by the disease or its treatment. ${ }^{20}$ This could be explained by the patients' learning process of coping with their disabilities as a result of their curative treatment. Particularly in cancer patients this phenomenon appears to play an important role.

Unravelling influencing factors is even more complicated due to the multiple treatment strategies used on an international level. This impedes clear comparisons across studies. In addition, especially in an ageing population future studies should be aware that age and ageing play an important role in HRQL. Future research might unravel the biopsychosocial factors associated with HRQL more specifically. To do this adequately, 
it might be beneficial to focus on specific quality of life domains, for example with the use of specific domain related questionnaires or more qualitative studies (e.g. focus groups, interviews) to bring the specific differences to light.

\section{What are the issues in the treatment of elderly patients?}

Using international guidelines and proposals from consensus meetings, we currently know which treatment strategy leads to the best outcomes. However, current national and international guidelines do not specifically focus on elderly patients and for most physicians it is a difficult group of patients to deal with. Elderly have other ideas in relation to life expectancy and health-related quality of life (HRQL) than younger patients. They are a heterogeneous group with various comorbidities and their physiological fitness varies between very fit and frail, unable to sustain any treatment. As mentioned earlier, increasing age is associated with an increased likelihood of noncurative treatment, but age alone should never be the primary reason for withholding treatment. Furthermore, it is important to realize that life expectancy is increasing globally. For example, for males who have reached the age of 80 , the life expectancy is at least 10 years for those who are in the fittest quartile, whilst the less fit quartile has an expectancy of less than 3.3 years. ${ }^{21}$ For women, these life-expectancies are even better. Thus fit elderly may benefit from the best oncological outcome in relation with their life expectancy. In Chapter $\mathbf{8}$ the available literature on elderly with rectal cancer was reviewed and the most important aspects were addressed.

Optimal treatment should start with an adequate workup prior to any treatment given in case of non-obstructive rectal cancer. ${ }^{22}$ Second, the frailty of the patient needs to be identified prior to treatment, because it is a predictor for post-operative complications and shorter life-expectancy. ${ }^{23,24}$ Frailty can be defined as "an elderly patient who is at heightened vulnerability to adverse health status change (such as hospitalization, mortality, nursing home admission) because of a multisystem reduction in reserve capacity". It is still widely discussed how to identify frailty in an individual patient. ${ }^{25,26}$

In case of suspected frailty, a referral to a geriatric oncologist is advised for a more comprehensive assessment and therapeutic interventions. After adequate staging and assessment of the patients' physiological condition and personal wishes, it is important that all patients are discussed in a multidisciplinary team meeting (MDT). A geriatrician may contribute to the point of view in these MDT meetings.

If a patient is considered fit for 'treatment', the current guidelines for rectal cancer offer the best chance for cure. Physiological fit elderly, irrespective of age can sustain neo-adjuvant treatment if managed properly. ${ }^{27}$ This means the use of short course radiotherapy in primary resectable cancer and chemoradiation in case of a locally advanced tumour. Nevertheless, there is an increase in morbidity and a sharp increase in post-operative mortality in elderly rectal cancer patients after surgery, indicating the 
importance of post-operative care in the elderly. Elderly need to receive the best possible post-operative support and need to be carefully monitored. Complications need to be tackled early and particularly in the case of anastomotic leakage, aggressive and early management should result in a low overall subsequent mortality. ${ }^{28}$ In addition, in the 'shared' decision making process the pros and cons of a stoma have to be outweighed against bowel continuity and the risk of anastomotic leakage. A permanent stoma is acceptable in the elderly population, particularly in those patients with a low rectal tumor, who will suffer more from the complications or functional problems of a low anastomosis.

In elderly rectal cancer patients shared decision making is the cornerstone for adequate treatment. In shared decision making, treating physicians need to be informed on their patients wishes and therapeutic interventions should be adapted to these needs and expectations. The most relevant interventions should be actively discussed in order to reach a common goal and thus a 'shared decision'. It is important to allow sufficient time to make the decision. After radiotherapy there is no need to rush for surgery. The waiting time after neoadjuvant strategies may help to make a better shared decision, which could be to observe for a while.

\section{Future perspectives}

In the present era, patients should no longer be at risk of inadequate diagnoses, resulting in under- or over staging, or receiving inappropriate treatment. From the patients' perspective, they will benefit most if treated by the physician with the best expertise in colorectal treatment. To do so, such a physician has to be expertly trained and have a large experience in order to treat patients. However on an individual basis, these physicians are only able to treat relatively small numbers of patients. Therefore, on a population-based and international level, patients will benefit more if there is not only an dedicated group of experts. But experts who share their expertise and therefore treatment should be based on guidelines formulated by and discussed with expert consensus panels (e.g. international guidelines). This allows high-quality patient care, irrespective to the region or country in which a treatment is offered. A shift in mind-set by physicians and experts should occur in order to achieve the best possible treatment for each patient. In Chapter 9 we addressed the importance of sharing expertise in colorectal cancer. In order to promote the use and benefit from expert opinions, initiatives such as the European consensus meeting and the Beyond TME collaborative, were international and worldwide guidelines created to share these thoughts and improve the overall quality of care nationally and internationally. We have to be aware that standardizing treatment is not necessarily the ultimate solution to optimized cancer care. As patients differ widely in pre-treatment performance status, disease stage, and many other important clinical demographical factors, 
physicians might do more harm rather than good by blindly implementing standardized treatment. With the use of guidelines it is possible to treat the majority of the patients, but physicians still have to individualise the treatment in their patients with comorbidities with regard to their ability to tolerate such treatments. In this era of standardization, registration, and auditing, patient-tailored treatment should not be neglected, and one should be aware that guidelines do not lead to a one-size-fits-all treatment.

Avoiding and decreasing morbidity, especially in the elderly, will require the development of new innovative strategies. The wait and see approach after clinical complete remission is an interesting development in rectal cancer treatment. Particularly for elderly patients, in whom surgery might result in significant morbidity and mortality, a less aggressive management with a wait and see policy is promising in selected patients. A complete response after radiochemotherapy is achieved in 15-25\% of the cases ${ }^{29,30}$ and could be the basis for a wait and see approach in the future. A Dutch study showed promising results with strict selection criteria, up-to-date imaging techniques, and follow-up. ${ }^{31}$ Currently, a wait and see policy is only applicable in carefully selected patients and future challenges are to achieve more frequent complete responses without increasing toxicity and the development of adequate follow-up strategies. Elderly patients considering non-invasive treatment, should be supported in the decision making process, in which evidence and tailored information on the wait and see policy is shared.

Another development in treatment regimens is the use of short course radiotherapy followed by a longer waiting period to definitive surgery. Results from the Swedish Rectal Cancer Registry showed promising results for short course radiotherapy and surgery after $>4$ weeks regarding downstaging and post-operative morbidity. ${ }^{32}$ The Stockholm III trial was conducted and randomized patients to either short course radiotherapy with direct or delayed surgery, or long course radiotherapy with delayed surgery. In the most recent interim analysis they report more downstaging and lower disease stage in the short course radiotherapy with delayed surgery group compared to immediate surgery group. ${ }^{33}$ They also report a high complete remission of $11.8 \%$ of the cases in the delayed surgery group. Reports from the long course radiotherapy with delayed surgery need to be awaited. This promising study is especially interesting for elderly in whom chemotherapy has an additional risk for morbidity and toxicity. Nevertheless, young patients could also fare well with this regimen.

Finally, the modern approach to rectal cancer should be a more individualised approach. Driven by international guidelines, the physician does not only need to inform the patient on the different treatment possibilities, oncological outcome and morbidity, but also on the influence of treatment on HRQL. This thesis provided more insight in age related differences in rectal cancer treatment and HRQL after treatment. It proposes a more individualised approach for a clinician, who is not only focussed on 
the optimal oncological outcome but also on functional results and subsequent quality of life of their patients. Ultimately, the optimal strategy in rectal cancer treatment should be shared decision making. 


\section{References}

1. IKNL Cijfers over kanker, http://www.cijfersoverkanker.nl/selecties/dataset_1/img56bb0a93ce659?language=en, accessed 2211-2015.

2. Meyer JE, Narang T, Schnoll-Sussman FH, Pochapin MB, Christos PJ, Sherr DL. Increasing incidence of rectal cancer in patients aged younger than $\mathbf{4 0}$ years: an analysis of the surveillance, epidemiology, and end results database. Cancer 2010;116:4354-9.

3. Davis DM, Marcet JE, Frattini JC, Prather AD, Mateka JJ, Nfonsam VN. Is it time to lower the recommended screening age for colorectal cancer? J Am Coll Surg 2011;213:352-61.

4. O'Connell JB, Maggard MA, Liu JH, Etzioni DA, Ko CY. Are survival rates different for young and older patients with rectal cancer? Dis Colon Rectum 2004;47:2064-9.

5. Ganapathi S, Kumar D, Katsoulas N, et al. Colorectal cancer in the young: trends, characteristics and outcome. Int J Colorectal Dis 2011;26:927-34.

6. Fairley TL, Cardinez CJ, Martin J, et al. Colorectal cancer in U.S. adults younger than 50 years of age, 1998-2001. Cancer 2006;107(5 Suppl):1153-61.

7. Endreseth $\mathrm{BH}$, Romundstad $\mathrm{P}$, Myrvold HE, Hestvik UE, Bjerkeset $\mathrm{T}$, Wibe A. Rectal cancer in the young patient. Dis Colon Rectum 2006;49:993-1001.

8. You YN, Dozois EJ, Boardman LA, Aakre J, Huebner M, Larson DW. Young-onset rectal cancer: presentation, pattern of care and long-term oncologic outcomes compared to a matched older-onset cohort. Ann Surg Oncol 2011;18:2469-76.

9. O'Connell JB, Maggard MA, Livingston EH, Yo CK. Colorectal cancer in the young. Am J Surg 2004; 187:343-8.

10. Smith C, Butler JA. Colorectal cancer in patients younger than 40 years of age. Dis Colon Rectum 1989; 32:843-6.

11. Chang GJ, Skibber JM, Feig BW, Rodriguez-Bigas M. Are we undertreating rectal cancer in the elderly? An epidemiologic study. Ann Surg 2007;246:215-21.

12. Dharma-Wardene MW, de GC, Au HJ, Hanson J, Hatcher J. Ageism in rectal carcinoma? Treatment and outcome variations. Int J Gastrointest Cancer 2002;32:129-38.

13. Schiphorst AH, Verweij NM, Pronk A, Hamaker ME. Age-related guideline adherence and outcome in low rectal cancer. Dis Colon Rectum 2014;57:967-75.

14. Surgery for colorectal cancer in elderly patients: a systematic review. Colorectal cancer collaborative group. Lancet 2000;356:968-74.

15. Balducci L. Geriatric oncology: challenges for the new century. Eur J Cancer 2000;36:1741-54.

16. Dekker JW, van den Broek CB, Bastiaannet E, van de Geest LG, Tollenaar RA, Liefers GJ. Importance of the first postoperative year in the prognosis of elderly colorectal cancer patients. Ann Surg Oncol 2011;18:1533-9.

17. Visser BC, Keegan H, Martin M, Wren SM. Death after colectomy: it's later than we think. Arch Surg 2009;144:1021-7.

18. Rutten HJ, den DM, Lemmens VE, van de Velde CJ, Marijnen CA. Controversies of total mesorectal excision for rectal cancer in elderly patients. Lancet Oncol 2008;9:494-501.

19. Ho VP, Lee Y, Stein SL, Temple LK. Sexual function after treatment for rectal cancer: a review. Dis Colon Rectum 2011;54:113-25.

20. Sprangers MA, Schwartz CE. Integrating response shift into health-related quality of life research: a theoretical model. Soc Sci Med 1999;48:1507-15.

21. Walter LC, Covinsky KE. Cancer screening in elderly patients: a framework for individualized decision making. JAMA 2001;285:2750-6.

22. Papamichael D, Audisio R, Horiot JC, et al. Treatment of the elderly colorectal cancer patient: SIOG expert recommendations. Ann Oncol 2009;20:5-16.

23. Fried LP, Tangen CM, Walston J, et al. Frailty in older adults: evidence for a phenotype. J Gerontol A Biol Sci Med Sci 2001;56:M146-56. 
24. Kristjansson SR, Nesbakken A, Jordhoy MS, et al. Comprehensive geriatric assessment can predict complications in elderly patients after elective surgery for colorectal cancer: a prospective observational cohort study. Crit Rev Oncol Hematol 2010;76:208-17.

25. Bellan van KG, Rolland Y, Bergman H, Morley JE, Kritchevsky SB, Vellas B. The I.A.N.A task force on frailty assessment of older people in clinical practice. J Nutr Health Aging 2008;12:29-37.

26. Martin FC, Brighton P. Frailty: different tools for different purposes? Age Ageing 2008;37:129-31.

27. Martijn H, Vulto JC. Should radiotherapy be avoided or delivered differently in elderly patients with rectal cancer? Eur J Cancer 2007;43:2301-6.

28. Khan AA, Wheeler JM, Cunningham C, George B, Kettlewell M, Mortensen NJ. The management and outcome of anastomotic leaks in colorectal surgery. Colorectal Dis 2008;10:587-92.

29. O'Neill BD, Brown G, Heald RJ, Cunningham D, Tait DM. Non-operative treatment after neoadjuvant chemoradiotherapy for rectal cancer. Lancet Oncol 2007;8:625-33.

30. Maas M, Nelemans PJ, Valentini V, et al. Long-term outcome in patients with a pathological complete response after chemoradiation for rectal cancer: a pooled analysis of individual patient data. Lancet Oncol 2010;11:835-44.

31. Maas M, Beets-Tan RG, Lambregts DM, et al. Wait-and-see policy for clinical complete responders after chemoradiation for rectal cancer. J Clin Oncol 2011;29:4633-40.

32. Pettersson D, Holm T, Iversen H, Blomqvist L, Glimelius B, Martling A. Preoperative short-course radiotherapy with delayed surgery in primary rectal cancer. Br J Surg 2012;99:577-83.

33. Pettersson D, Lorinc E, Holm T, Iversen H, Cedermark B, Glimelius B, Martling A. Tumour regression in the randomized Stockholm III trial of radiotherapy regimens for rectal cancer. Br J Surg 2015;102:972-8. 
Samenvatting 
Het coloncarcinoom (dikke darm kanker) is een van de meest voorkomende vorm van kanker in Nederland. Ongeveer 1/3 van deze carcinomen betreft het rectumcarcinoom (endeldarm kanker). De incidentie is de afgelopen jaren toegenomen, waarbij er in 20154.342 nieuwe gevallen zijn ontdekt. Het rectumcarcinoom wordt met name gediagnosticeerd bij de oudere patiënten, waarvan meer dan $75 \%$ ouder is dan 60 jaar. Het rectumcarcinoom komt echter niet alleen bij ouderen voor, ook bij jongeren wordt een stijgende incidentie gezien. Door de vergrijzing is de verwachting dat de incidentie van het rectumcarcinoom zal toenemen over de jaren.

Sinds de jaren ' 90 is de behandeling van het rectumcarcinoom sterk verbeterd. Zowel het verbeteren van de chirurgische techniek (TME) als het toevoegen van neoadjuvante behandeling (radiotherapie / chemoradiatie) hebben bijgedragen aan een betere overleving. Het rectumcarcinoom is hierbij een curabele ziekte geworden. In Chapter 2 wordt een overzicht gegeven over hoe de moderne behandeling van het rectumcarcinoom tot stand is gekomen. In dit hoofdstuk wordt niet alleen aandacht geschonken aan de ontwikkelingen op chirurgisch gebied, maar ook aan de verbeteringen in de multidisciplinaire aanpak van het rectumcarcinoom, waarbij optimale beeldvorming, pathologie en samenwerking tussen alle disciplines noodzakelijk is.

Ondanks alle verbeteringen in radio- en chemotherapie, is chirurgie tot op heden de belangrijkste speler in de behandeling. Voor het voorkomen van een lokaal recidief is een radicale resectie (schone snijranden) essentieel. Door het toevoegen van neoadjuvante behandeling is het mogelijk om bij lokaal voortgeschreden carcinomen (ingroei in andere structuren) orgaan sparend te kunnen opereren. Helaas is bij een deel van de patiënten een uitgebreidere resectie met medenemen van andere organen (bv. blaas/prostaat/vagina) noodzakelijk om tumorvrije snijranden te verkrijgen. Tijdens deze operatie wordt een definitief colo- en/of urostoma aangelegd. Dit zijn majeure operaties met kans op ernstige complicaties.

Dit proefschrift zal ingaan op enkele belangrijke vraagstukken in de behandeling van het rectumcarcinoom. Het geeft inzicht in verschillen tussen leeftijdsgroepen (jong/oud) ten aanzien van tumorkarakteristieken, behandeling en overleving. Daarnaast is er aandacht voor invloed van deze tumoren en diens behandeling op de kwaliteit van leven van deze patiënten. Het doel van dit proefschrift is om de behandeling van het rectumcarcinoom meer te individualiseren, kijkend naar de best mogelijke oncologische uitkomst en tevens behoud van kwaliteit van leven voor alle rectumcarcinoom patiënten. 


\section{Leeftijdsverschillen in de behandeling van het rectumcarcinoom}

Zoals eerder gezegd is er niet alleen een stijgende incidentie van het rectumcarcinoom bij ouderen, maar wordt dit ook gezien bij patiënten $\leq 40$ jaar. In de huidige literatuur is er weinig bekend over de kenmerken van deze tumoren en de uitkomst van behandeling in deze groep jonge patiënten. In Chapter 3 zijn de verschillen in tumorkarakteristieken, behandeling en overleving bestudeerd van 37.000 patiënten. Hiervoor zijn data van de Nederlandse kankerregistratie (NKR) gebruikt. Het NKR registreert sinds 1989 alle nieuwe patiënten met een vorm van kanker.

Voor deze studie zijn meer dan 37.000 patiënten met een rectumcarcinoom in de leeftijd tot 70 jaar geïncludeerd. Deze patiënten zijn verdeeld in een "jongere" groep ( $\leq 40$ jaar) en een "oudere" groep (41-70 jaar) en met elkaar vergeleken. Een belangrijke bevinding is dat jonge patiënten zich vaker presenteren met prognostisch ongunstigere tumoren in een gevorderd stadium t.o.v. oudere patiënten. Ondanks deze meer kwaadaardige tumoren is overleving tussen beide groepen gelijk, en een jongere leeftijd was zelfs voorspellend voor een betere overleving. Dit wil zeggen dat de behandeling die jongeren ondergaan in ieder geval resulteert in een gelijke of mogelijk betere overleving vergeleken met oudere patiënten, ondanks de prognostisch ongunstige tumorkenmerken. Onze studie liet ook een toegevoegde waarde zien van adjuvante chemotherapie voor de overleving van jonge patiënten, echter hier dient nader onderzoek naar gedaan te worden alvorens dit te standaardiseren, aangezien selectiebias waarschijnlijk een grote rol speelt.

Bij het lokaal voortgeschreden carcinoom is vaak uitgebreidere behandeling nodig. Deze behandeling gaat gepaard met een grote morbiditeit, hierdoor lopen ouderen patiënten het risico niet curatief behandeld te worden. Chapter $\mathbf{4}$ geeft meer inzicht in de morbiditeit en mortaliteit van de intensieve behandeling bij uitgebreidere tumoren (lokaal voortgeschreden of een lokaal recidief). Hiervoor zijn alle patiënten geanalyseerd met een lokaal voortgeschreden (cT4) of lokaal recidief, geopereerd in het Catharina Ziekenhuis tussen 2005-2013. Van de cT4 patiëntengroep <75 jaar had $56 \%$ en van de groep $\geq 75$ jaar $69 \%$ tenminste één complicatie. Voor de lokaal recidief groep was dit respectievelijk $75 \%$ en $96 \%$, voor beide leeftijdsgroepen. Ondanks deze hoge morbiditeit is de 1-maands mortaliteit voor beide groepen laag. Voor de cT4 patiënten >75 jaar is dit echter een zware onderschatting voor de 1-jaarsoverleving, waarbij er een 1-jaars sterfte is van $20 \%$ ten opzichte van $5 \%$ in de jongere leeftijdsgroep. Bij patiënten behandeld voor een lokaal recidief is de postoperatieve sterfte ook een onderschatting van de 1-jaars sterfte, maar deze is niet leeftijdsafhankelijk en waarschijnlijk ziekte gerelateerd. Chapter 4 laat zien dat ouderen in staat zijn om deze uitgebreidere behandelingen te doorstaan. Er moet echter meer aandacht komen voor verbetering in 1-jaars overleving van oudere patiënten. Toekomstig onderzoek zou zich kunnen richten op methodes voor betere 
patiëntselectie of optimaliseren van de perioperatieve conditie en voedingsstatus in deze specifieke patiëntengroep.

\section{Kwaliteit van leven na behandeling van het rectumcarcinoom}

Doordat de behandeling van het rectumcarcinoom over de afgelopen decennia sterk is verbeterd wordt kwaliteit van leven na behandeling steeds belangrijker. Immers, we moeten streven naar die behandeling die niet alleen de beste kans op overleving biedt, maar ook met behoud van kwaliteit van leven. Om de invloed van tumorstadium, chirurgie en stoma te bestuderen op de kwaliteit van leven (QOL) zijn een drietal studies verricht met behulp van gevalideerde vragenlijsten. In Chapter $\mathbf{5}$ wordt de QOL van patiënten met verschillende tumorstadia met elkaar vergeleken. Patiënten die zijn behandeld voor een lokaal recidief rapporteerden een lagere kwaliteit van leven vergeleken met lokale tumoren en lokaal voortgeschreden tumoren. Als onderdeel van $\mathrm{QOL}$ is ook gekeken naar seksueel functioneren. Alle patiëntengroepen rapporteerden een slechter seksueel functioneren vergeleken met gezonde individuen. Opmerkelijk was dat slechts een enkeling hiervoor hulpmiddelen gebruikte. Daarnaast was minder dan $50 \%$ van de mannen en $35 \%$ van de vrouwen seksueel actief na behandeling.

Zoals eerder vermeld is uitgebreidere chirurgie vaker nodig bij lokaal voortgeschreden tumoren, omdat deze zich niet houden aan anatomische grenzen en ingroeien in andere structuren. Doordat het rectum gelokaliseerd is in het kleine bekken, waar de urogenitale structuren dicht tegen het rectum zijn aangelegen, zijn deze structuren het vaakst aangedaan. Met neo-adjuvante behandeling wordt gepoogd deze tumoren kleiner te maken, zodat meer orgaan sparende operaties mogelijk zijn. Bij een deel van de patiënten is echter nog steeds uitgebreidere chirurgie nodig, met medenemen van verschillende tot alle urogenitale organen. In Chapter 6 wordt de invloed van deze uitgebreidere resecties op de kwaliteit van leven bestudeerd. Voor deze studie zijn patiënten met een lokaal voortgeschreden carcinoom geselecteerd en onderverdeeld in een rectumresectie (TME-chirurgie) met of zonder medenemen van andere organen. Om het effect van leeftijd nader te bestuderen werden patiënten onderverdeeld in twee leeftijdsgroepen. Uitgebreidere chirurgie leidde niet tot een mindere kwaliteit van leven ten opzichte van een standaard rectumresectie. Bij patiënten $<70$ jaar resulteerde een uitgebreidere resectie in een slechter lichaamsbeeld ten opzichte van de rest van de patiëntgroepen. Verrassend was dat seksueel functioneren niet gerelateerd was aan mate van chirurgie; een uitgebreidere resectie resulteerde niet in een verminderde seksuele functie t.o.v. een standaard resectie. Jonge mannen na standaard chirurgie rapporteerden de minste seksuele stoornissen.

In Chapter 7 is de invloed van een stoma op de kwaliteit van leven bestudeerd. Afhankelijk van de hoogte van de tumor in het rectum kan er wel of geen anastomose (verbinding) aangelegd worden, resulterend in een tijdelijk of definitief stoma. Indien er 
een anastomose is aangelegd en er treedt lekkage op, dan kan dit ernstige complicaties met zich meebrengen. In het ernstigste geval resulteert dit in overlijden van de patiënt. Het is bekend dat naadlekkages bij ouderen ernstigere gevolgen kunnen hebben dan bij jongere patiënten. Om ernstige complicaties te voorkomen zou gekozen kunnen worden om geen anastomose te maken en direct een definitief stoma aan te leggen. Gedacht wordt dat een stoma de kwaliteit van leven ernstig beïnvloedt. Om de invloed van een stoma op de kwaliteit van leven te bestuderen hebben we bij patiënten met een lage rectumtumor geïncludeerd en vragenlijsten bij hen afgenomen. De patiënten zijn wederom verdeeld in twee leeftijdsgroepen (<70 en $\geq 70$ jaar). Patiënten met een stoma rapporteerden een gelijkwaardige kwaliteit van leven ten opzichte van patiënten zonder stoma. Daarnaast rapporteerden rectumcarcinoom patiënten, ongeacht het hebben van een stoma, een nagenoeg gelijkwaardige kwaliteit van leven ten opzichte van gezonde individuen. Rekening houdend met de risico's op een naadlekkage bij oudere en kwetsbare patiënten is een definitief stoma een goede mogelijkheid, gekeken naar de kwaliteit van leven en het voorkomen van een naadlekkage.

Op basis van dit proefschrift kan worden geconcludeerd dat rectumcarcinoom patiënten na behandeling een nagenoeg gelijkwaardige kwaliteit van leven hebben t.o.v. van de normale bevolking, ongeacht de aanwezigheid van stoma, de uitgebreidheid van de tumor of de uitgebreidheid van de behandeling. Op individueel niveau zullen echter zeker beperkingen zijn die de kwaliteit van leven benadelen, maar waarschijnlijk speelt het "overleven" ongeacht de beperkingen (survival bias) een grote rol. De patiënten zijn hoogstwaarschijnlijk dankbaar dat ze van de kanker zijn genezen en leren omgaan met de beperkingen die de behandeling met zich mee heeft gebracht.

In dit proefschrift wordt opnieuw duidelijk dat de behandeling van het rectumcarcinoom een enorme negatieve impact heeft op seksueel functioneren. Behandelaars zouden meer aandacht aan dit onderwerp moeten schenken. Het bespreken of signaleren van potentiele seksuele stoornissen zou onderdeel moeten worden van de dagelijkse praktijk.

\section{De behandeling van het rectumcarcinoom bij de oudere patiënt}

Door nationale en internationale richtlijnen weten we welke behandelingen leiden tot de beste overleving. In deze richtlijnen wordt de oudere patiënt vaak buiten beschouwing gelaten. Ouderen zijn kwetsbaar door multipele comorbiditeiten, hierdoor lopen zij het risico om niet curatief behandeld te worden. Daarnaast hebben oudere patiënten een andere toekomstvisie dan jongere patiënten. Het is invoelbaar dat voor ouderen een langere levensverwachting minder waarde heeft en dat zij meer waarde hechten aan behoud van kwaliteit van leven zonder lichamelijke beperkingen en grote chirurgie. Jongere patiënten daarentegen zullen eerder streven naar de best mogelijke overleving, ongeacht de beperkingen die de behandeling met zich mee kan 
brengen. Voor de clinicus is de oudere patiënt dan niet eenvoudig om goed te behandelen. Dit komt door de grote variatie in conditie en wensen van de oudere patiënten. In Chapter $\mathbf{8}$ worden, gebruikmakend van de beschikbare literatuur, belangrijke vraagstukken in de behandeling bij ouderen besproken, waarbij het komen tot een gezamenlijk besluit, na goede voorlichting het belangrijkst aspect van de behandeling is.

\section{De toekomstige behandeling van het rectumcarcinoom}

In Chapter 9 wordt een visie gegeven over hoe de behandeling van het rectumcarcinoom zich zal ontwikkelen. Er wordt ingegaan op de verbetering van de zorg rondom het rectumcarcinoom door nationale en internationale registratie van deze behandeling te vergelijken. Door de ontwikkelingen in diagnostiek, behandeling en het ontstaan van richtlijnen, mogen patiënten niet meer het risico lopen op foutieve diagnoses en inadequate behandeling. Vanuit het perspectief van de patiënt zou het ideaal zijn dat iedere patiënt behandeld wordt door een expert. Dit is echter onmogelijk, omdat één expert nooit in staat is iedereen te behandelen. Voor uitgebreidere tumoren is het verwijzen naar een expert centrum zeker aangewezen, maar een niet lokaal voortgeschreden tumor kan uitstekend in het eigen centrum behandeld worden. Initiatieven als "Beyond the TME collaborative" of European consensus meetings brengen experts bijeen om kennis uit te wisselen en gezamenlijke richtlijnen te maken. Door al deze initiatieven is het mogelijk dat elke arts zijn patiënt behandelt volgens "experts" opinion, resulterende in de best mogelijke uitkomst.

\section{Conclusie}

De moderne rectumcarcinoom behandeling zou een individueel patiënt gerichte behandeling moeten zijn. Bevlogen door (inter-)nationale richtlijnen zou de behandelend arts zijn patiënt moeten informeren over de verschillende behandelingen en tevens de invloed op overleving en kwaliteit van leven. Dit proefschrift geeft meer inzicht in deze belangrijke vraagstukken. Uiteindelijk ligt de crux van een goede behandeling in gezamenlijke besluitvorming. 
Valorization 
Colorectal cancer is the second most frequent type of cancer in male and third most common malignancy in women. Rectal cancer mainly affects middle-aged and elderly individuals, with more than $75 \%$ of patients being diagnosed at age 60 years or older. Nevertheless, also younger patients, under 40 years of age, are increasingly affected.

The rectum is localized in the pelvis and is surrounded by uro- and genital organs. To date, the only curative treatment for rectal cancer is surgery, although improvements are made with less invasive approaches (e.g. wait and see). In early stage rectal cancer (cT1-T2), less invasive treatment is possible with local tumor resection (e.g. transanal endoscopic microsurgery (TEM)). In case of more advanced disease (cT2+/N+), neoadjuvant radiotherapy and total mesorectal excision (TME) surgery is needed in order to achieve curation. In case of locally advanced disease (LARC), the tumour involves surrounding structures and intensified treatment regimens are needed to downsize the tumor. If after neo-adjuvant treatment, the tumor still invades the surrounding structures, extended resections are needed with an en-bloc removal of the affected organs. This often results in a colo- and/or urostomy. In addition, with removal of genital organs, these resections affect urologic and sexual function. But also in nonadvanced disease stage (T2+), urologic and sexual dysfunction is seen after surgery. For both the clinician and the patient, knowledge on the influence of treatment strategies on patients' health related quality of life (HRQL) is extremely important.

Nowadays, patient-reported outcome and quality of life are increasingly important. However, the last decades research on primary or recurrent rectal cancer focused primarily on improving oncological outcome. In addition, HRQL was not actively discussed during the past international expert consensus meetings. The current approach to rectal cancer requires knowledge of the influence of the treatment, on the patients' HRQL. Patients need to be well informed about the choices that can be made regarding their treatment and they should participate actively in the decision making process. In this thesis, the effect of tumour stage and subsequent treatment, extent of surgery and influence of a permanent stoma on HRQL was investigated. In general, we can conclude that despite the intensive treatment regimens and major surgery these patients have to endure, their HRQL after treatment can be considered good. In addition, a permanent stoma does not influence HRQL in a negative way. In patients with a low rectal tumor, who will suffer more from the complications or functional problems of a low anastomosis, a permanent stoma needs to be discussed prior to surgery. Especially in frail or elderly patients, a permanent stoma is feasible while maintaining a good HRQL.

The negative impact of curative treatment on sexual function, as found in this thesis, calls for further attention and awareness to alleviate this problem in sexually active patients. For optimal consultation, sexual dysfunction due to surgery should be actively discussed during a patients visit.

With the use of data from the Netherlands Cancer Registry, age related differences in treatment and outcome were investigated in this thesis. Young patients are less 
commonly seen during daily practice and less research on tumor characteristics and outcome of treatment in this particular patient group is available. We observed that young patients present with more advanced stage of disease and have more unfavourable tumor characteristics compared to middle aged-aged patients. Despite these unfavourable characteristics, survival rates are equal and young age is even a prognosticator for better survival. Young patients received adjuvant chemotherapy more often compared to middle-aged patients. This was associated with improved survival in young patients with pN1 or stage III disease. Nevertheless, the additional survival benefit of adjuvant chemotherapy has to be interpreted with caution, and further research will be needed to assess the effect of adjuvant chemotherapy in young rectal cancer patients.

Elderly patients are at risk of not being treated according to the national guidelines. They are a difficult heterogeneous group of patients with often multiple comorbidities and often poor clinical condition. Due to their age and physical condition, they are more often treated with a non-curative intent. Furthermore, they are also underrepresented in clinical studies and current guidelines do not focus on elderly patients. Therefore, evidence on how to treat the elderly patient with rectal cancer is scarce.

Little is known about how elderly patients with LARC or LRRC endure intensive treatment regimens including, neo-adjuvant therapy followed by major rectal surgery and multi-visceral resections. In our study, including only LARC or LRRC patients, morbidity was high regardless of age in LARC patients. In LRRC, differences in morbidity were observed but were not statistically different. One-month mortality was equal for both treatment groups and regardless of age. However, after one year, an almost threefold increase in mortality was observed in elderly LARC patients compared to younger LARC patients. A similar increase in mortality was seen in LRRC patients, but this was not age related. This study highlights that elderly patients can be treated according to the guidelines with neo-adjuvant treatment and major surgery, but improvements are needed in patient selection or ( $p r e / r e)$ habilitation programs in order to lower the one-year post-operative mortality. Particularly in advanced cases there is enough time for optimizing the patient prior to surgery in the interval between neoadjuvant treatment and definitive surgery. Future studies focusing on the role of a prehabilitation program during the waiting period on post-operative outcome in elderly with LARC and LRRC cases are warranted.

This thesis has addressed several issues in HRQL and morbidity of treatment between various age groups. We were not able to detect major differences in quality of life with general HRQL questionnaires. Future research should focus more on the differences between pre- and post-treatment HRQL and the influence of sexual support on general HRQL. Furthermore adequate selection of elderly patients with awareness of the 
morbidity of this locally progressive disease in relation to the impact of surgery on morbidity and mortality could improve outcome and quality of life.

Finally, the modern approach to rectal cancer should be a more individualised approach. Driven by international guidelines, the physicians do not only need to inform their patients on the different treatment possibilities, oncological outcome and morbidity, but also on the influence of treatment on HRQL. This thesis provides insight in age related differences in rectal cancer treatment and HRQL after treatment. It provides an insight in a more individualised treatment approach for daily practice. We should not only focus on optimal oncological outcome but also on functional results and subsequent quality of life of our patients after treatment. Ultimately, the optimal strategy in rectal cancer treatment should be shared decision making. 
Dankwoord 
Eindelijk, het proefschrift is af. $\mathrm{Nu}$ is het mijn tijd om enkele mensen speciaal te bedanken. Zonder de directe en indirecte bijdrage van een ontzettend grote groep mensen was dit proefschrift niet tot stand gekomen. Mijn dank is dan ook groot aan iedereen die heeft bijgedragen aan dit proefschrift.

Ik wil alle patiënten bedanken die hebben geparticipeerd in de verschillende studies. Dankzij hen zijn wij in staat de behandeling van toekomstige patiënten naar een hoger niveau te tillen.

Prof. dr. Rutten, beste Harm, wat heb je me veel geholpen tijdens dit proefschrift. Behalve mijn promotor was je mijn vraagbaken. Ik kon voor alles bij je terecht en ieder artikel heb je van begin af aan begeleid. De onderzoekbespreking met afhaal chinees werd een traditie waarbij het altijd onwijs gezellig was en het bespreken van onderzoek meer bijzaak werd. Ik kan niet anders dan je bedanken voor alle inspiratie die je me hebt gegeven en hoop in de toekomst nog veel van jouw chirurgische skills te leren.

Dr. de Hingh, beste Ignace, als jij me niet had aangenomen als semi-arts was dit proefschrift er nooit geweest. Ik weet nog goed hoeveel rood ik zag op de gecorrigeerde versie van ons eerste artikel. Dank je wel voor je ontzettende kritische blik bij al onze studies. Gelukkig zullen we elkaar, ook na dit proefschrift nog regelmatig zien om een biertje te drinken.

Dr. Nieuwenhuijzen, beste Grard, ik wilde chirurg worden en jij had vanaf het begin af aan hiervoor het plan. Eerst als ANIOS aan de slag in het Catharina Ziekenhuis, tegelijkertijd schrijven aan dit proefschrift en dan in opleiding voor de chirurgie. Ik kan niet anders zeggen dat dit plan een meesterzet was. Dankzij jouw inspanningen heb ik het allemaal voor elkaar gekregen. Bedankt voor al je hulp en betrokkenheid, zowel tijdens dit proefschrift als ook tijdens de opleiding.

Co-auteurs, ik had dit proefschrift niet zonder jullie hulp en kritische blik kunnen voltooien. Een aantal wil ik in het bijzonder bedanken. Rob, Liza en Valery, bedankt voor jullie hulp bij het analyseren van de NKR data. Melissa, dank voor de begeleiding en analyses tijdens mijn eerste studie; jij moest me daadwerkelijk alle statistiek uitleggen. Marjan, ontzettend bedankt voor al je hulp en gezelligheid tijdens de kwaliteit van leven studies. Sietske, dank voor je hulp bij het verzamelen van de data van de lokaal recidieven: nu jij nog! Geert, bedankt voor je hulp bij mijn laatste studie.

De leden van de beoordelingscommissie, Prof. dr. Stassen, Prof. dr. Beets, Prof. dr. de Wilt en dr. Maas, hartelijk dank voor het kritisch lezen en beoordelen van mijn proefschrift en deelname aan de corona. 
Maatschap chirurgie van het Catharina Ziekenhuis, beste Harm, Grard, Ignace, Simon, Misha, Frans, Alex, Jean-Paul, Gust, Joep, Marc, Philippe en Yvonne. Dankzij jullie energie en betrokkenheid heb ik me als een vis in het water gevoeld tijden mijn semiarts, ANIOS en AIOS tijd. Ik heb ontzettend veel van jullie mogen leren. De jaarlijkse winterbijscholing blijft een feest. Gelukkig mag ik nog 2 jaar terugkomen om mijn opleiding bij jullie af te maken.

Arts-assistenten en oud assistenten Catharina ziekenhuis, wat hebben we een hoop plezier met zijn allen. De hechte band op en buiten de werkvloer zorgt voor meer dan voldoende afleiding. Het wordt weer tijd om plezier te maken!

Arts-assistenten MUMC+, jullie zijn een mooie club collega's. Ook in de grote academie weten we een hoop plezier te maken. De regelmatige X-box matches zijn fantastisch! Nog even blijven oefenen jongens!

Alle medewerkers van de poli chirurgie, OK, SEH en verpleegkundigen Catharina Ziekenhuis en MUMC+, veel dank voor de dagelijkse gezelligheid.

Intensivisten Catharina Ziekenhuis, beste Arnout, Alex, Marc, Herman, Marco, Dick, Femke, Vesna, Erik en Ingeborg, jullie hebben mijn chirurgische blik weten te verbreden. Dank je wel voor de vele leermomenten op de intensive care.

Petri! Het was altijd feest op het werk en nu nog steeds daarbuiten: lekker guilty pleasures knallen!

Dit proefschrift was er nooit gekomen zonder de steun van al mijn vrienden en familie. Een aantal wil ik nog in het bijzonder bedanken.

Mijn studievriendjes Pim, Petson, Lokien, Robert-Jan, Willem. Niet alleen een fantastische tijd tijdens geneeskunde, in de pretflat maar ook daarbuiten hebben we nog steeds een hoop plezier samen! Thanks boys!

Just en Matthijs, mijn oudste vriendjes! Samen opgegroeid in Nuenen waar we van kinds af aan veel mooie avonturen beleefd hebben. Nu allemaal druk met onze eigen carrières maar gelukkig weten we toch nog regelmatig tijd te maken voor een mooie avond. Wanneer is de volgende?

Mijn Lorentz maatjes, Bart, Suus en Charlotte. Allemaal niet weg te krijgen uit Eindhoven en nog steeds traditiegetrouwe carnavalsvierders!

Leden van het plezierteam, oa. Poodt, Daan, Bou, Guus, Marijn en Stan. Van huisfeestjes, stapavonden tot festivals het is altijd alles geven! 
Beste Tom, het blijft een eeuwige strijd wie de mooiste man van Nederland is. We hebben sinds het eerste jaar geneeskunde een hechte vriendschap opgebouwd. Ook al wonen we niet bij elkaar om de hoek en bellen we altijd ongelegen, toch weten we elkaar regelmatig te vinden voor een biertje en sterke verhalen.

Lieve Opa Theo en Oma Bep, ik weet dat jullie trots op me zouden zijn. Ik denk aan jullie.

Lieve familie, Opa Sjef, Christa, Erik, Ilse, Arthur, Anouk, Renato en Ewan. Ik mag me gelukkig prijzen met zo'n fijne familie!

Lieve Quintin en Layla, mijn lieve broertje en zusje. Jullie enthousiasme en energie is geweldig! Door jullie blijft het een feestje om thuis te komen. Ik ben ongelofelijk benieuwd naar jullie toekomst, jullie kunnen altijd op mij rekenen.

Lieve Tatiàna, mijn grote zus. Lief en leed hebben we met elkaar gedeeld. Trots op wat je allemaal hebt bereikt in je uppie! Nu is het jouw tijd om te gaan genieten van wat komen gaat.

Lieve Papa, Marie-Jose, Kiky en Puck, bedankt voor jullie onvoorwaardelijke steun en liefde. Op onze dagelijkse verplichte belletjes om stipt 20u: "nog geopereerd?" "Hoe is het met je werkstuk?" kan ik eindelijk antwoorden: Nou pa... Het "werkstuk" is eindelijk af!

Lieve Mama en Walter, het altijd warme en gezellige nest zorgde ervoor dat ik maar al te graag thuis bleef wonen, al speelden de Oostenrijkse schnitzels ook een hele belangrijke rol. Jullie hebben me gemotiveerd om het beste uit mezelf te halen. Ik hou van jullie.

Emiel en Niels, mijn beste vriendjes. Wat hebben we geweldige dingen beleefd. Mooi dat jullie keer op keer de boot in gaan met een weddenschapje! Jullie zijn fantastische kerels. Een eer dat jullie mijn paranimfen zijn. Ik hoop dat de VvAA op de hoogte is van het feestje vanavond...

Beste Thomas, al vanaf geneeskunde volgen we het zelfde pad. Mooi dat we niet alleen collega's en onderzoeksmaatjes zijn, maar bovenal hele goede vrienden. We hebben het vaak over deze dag gehad en hoe cool het zou zijn om dit samen te vieren. We hebben het mooi voor elkaar kerel!

Liefste Sas, de liefde van mijn leven, jouw onuitputbare energie, zorgzaamheid en liefde is verslavend. Het is een feestje met jou aan mijn zijde. Lieve schat, jij bent fantastisch! 
List of publications 
Orsini RG, Thong MS, van de Poll-Franse LV, Slooter GD, Nieuwenhuijzen GA, Rutten HJ, de Hingh IH. Quality of life of older rectal cancer patients is not impaired by a permanent stoma. Eur J Surg Oncol 2013;39(2):164-170.

Orsini RG, Wiggers T, De Ruiter MC, Quirke P, Beets-Tan RG, van de Velde CJ, Rutten HJ. The Modern anatomical surgical approach to localized rectal cancer. EJC Suppl. 2013;11:60-71.

Traa MJ, Orsini RG, Den Oudsten BL, De Vries J, Roukema JA, Bosman SJ, Dudink RL, Rutten HJ. Measuring the health-related quality of life and sexual functioning of rectal cancer patients: does type of treatment matter? Int J Cancer 2013;134:979-987.

van Steenbergen LN, de Hing IH, Rutten HJ, Rijk MC, Orsini RG, Coebergh JW, Lemmens VE. Large variation between hospitals in follow-up for colorectal cancer in southern Netherlands. Int J Colorectal Dis 2013;28(9):1257-1265.

Vermeer TA, Orsini RG, Rutten HJ. Surgery for rectal cancer: what is on the horizon? Curr Oncol Rep 2014;16:372.

Vermeer TA, Orsini RG, Daams F, Nieuwenhuijzen GA, Rutten HJ. Anastomotic leakage and presacral abscess after rectal cancer surgery: Incidence, Risk Factors and Treatment. Eur J Surg Oncol 2014;40(11):1502-1509.

Orsini RG, Traa MJ, Vermeer TA, Nieuwenhuijzen GA, de Hingh IH, Rutten HJ. Does extended surgery influence health related quality of life in rectal cancer patients? Dis Colon Rectum 2015;58(2):179-185.

Traa MJ, Braeken J, De Vries J, Roukema JA, Orsini RG, Den Oudsten BL. Evaluating quality of life and response shift from a couple-based perspective: a study among patients with colorectal cancer and their partner. Qual Life Res 2015;24(6):1431-1441

Orsini RG, Verhoeven RH, Lemmens VE, van Steenbergen LN, de Hingh $I H$, Nieuwenhuijzen GA, Rutten HJ. Better survival for young rectal cancer patients, despite poorer morphology and more advanced stage. Eur J Cancer 2015;51(13):1675-1682.

Orsini RG, Kristjansson SR, Rutten HJ. Rectal cancer treatment in the elderly. W.E. Longo et al (eds.) Modern Management of Cancer of the Rectum $2^{\text {nd }}$ ed., 2015. SpringerVerlag London. 385-403. 
Vermeer TA, Orsini RG, Nieuwenhuijzen GA, Rutten HJ, Daams F. Emergency stoma placement in advanced rectal cancer: a practical guideline. Eur J Surg Oncol. 2016; 42(2):273-280.

Orsini RG, Rutten HJT. What are the issues in the treatment of elderly patients? Accepted, Multidisciplinary Management of Rectal Cancer-Questions and Answers. $2^{\text {nd }}$ ed., 2017 Springer-Verlag Berlin Heidelberg. 
Curriculum Vitae 


\section{Curriculum Vitae}

Ricardo Giovanni Orsini was born on December $24^{\text {th }} 1986$ in Geldrop, The Netherlands. After graduating from the Lorentz Casimir Lyceum in 2005 he started his medical study at the University of Maastricht. At the end of his study he did his final internship at the department of Surgery of the Catharina Hospital Eindhoven. Under supervision of Prof. dr. H.J.T. Rutten, dr. de I.H.J.T. Hingh and dr. G.A.P. Nieuwenhuijzen the first study of this thesis was conducted. After obtaining his medical degree in 2011 he started working as a resident not in training, first at the department of surgery and later on at the Intensive Care department of the Catharina Hospital Eindhoven. Alongside his clinical residencies he was actively involved in rectal cancer research and continued on his PhD project "Age related differences and quality of life in rectal cancer surgery". In January 2014 he started his surgical training program at the Catharina Hospital Eindhoven under supervision of dr. G.A.P. Nieuwenhuijzen and dr. S.W. Nienhuijs. Currently he is in training at the Maastricht University Medical Center under supervision of Prof. dr. L.P.S. Stassen. Next year he will return to the Catharina Hospital Eindhoven where he will finish his surgical training program at the end of 2019. Ricardo lives in Eindhoven together with Saskia Thomassen. 
